\title{
Lamin interA/Ctors: from the premature to senescence
}

Citation for published version (APA):

Kubben, L. P. (2011). Lamin interA/Ctors: from the premature to senescence. [Doctoral Thesis, Maastricht University]. Maastricht University. https://doi.org/10.26481/dis.20110126lk

Document status and date:

Published: 01/01/2011

DOI:

10.26481/dis.20110126lk

Document Version:

Publisher's PDF, also known as Version of record

\section{Please check the document version of this publication:}

- A submitted manuscript is the version of the article upon submission and before peer-review. There can be important differences between the submitted version and the official published version of record.

People interested in the research are advised to contact the author for the final version of the publication, or visit the DOI to the publisher's website.

- The final author version and the galley proof are versions of the publication after peer review.

- The final published version features the final layout of the paper including the volume, issue and page numbers.

Link to publication

\footnotetext{
General rights rights.

- You may freely distribute the URL identifying the publication in the public portal. please follow below link for the End User Agreement:

www.umlib.nl/taverne-license

Take down policy

If you believe that this document breaches copyright please contact us at:

repository@maastrichtuniversity.nl

providing details and we will investigate your claim.
}

Copyright and moral rights for the publications made accessible in the public portal are retained by the authors and/or other copyright owners and it is a condition of accessing publications that users recognise and abide by the legal requirements associated with these

- Users may download and print one copy of any publication from the public portal for the purpose of private study or research.

- You may not further distribute the material or use it for any profit-making activity or commercial gain

If the publication is distributed under the terms of Article $25 \mathrm{fa}$ of the Dutch Copyright Act, indicated by the "Taverne" license above, 


\section{Lamin interA/Ctors:}

From the premature to senescence 
ISBN 978-90-6464-428-3

(C) Nard Kubben, Munstergeleen 2011

Printing: GVO printers \& designers B.V. | Ponsen \& Looijen 


\title{
Lamin interA/Ctors:
}

\section{From the premature to senescence}

\author{
PROEFSCHRIFT \\ Ter verkrijging van de graad van doctor \\ aan de Universiteit Maastricht, \\ op gezag van de Rector Magnificus, \\ Prof. Mr. G.P.M.F. Mols, \\ volgens het besluit van het College van Decanen, \\ in het openbaar te verdedigen \\ op woensdag 26 januari 2011 om 16.00 uur \\ door \\ Leonard Pieter Kubben \\ Geboren op 18 december 1981 te Geleen
}




\section{Promoter}

Prof. dr. Y.M. Pinto

\section{Co-promotores}

Dr. T. Misteli

Dr. ir. J.W. Voncken

\section{Assessment Committee}

Prof. dr. H.J.G.M. Crijns (chairman)

Prof. dr. M. Post

Prof. dr. P.M. Steijlen

The research described in this thesis was supported by a grant of the Netherlands Heart Foundation (NHF2000.130, 2002T016), ZonMW, NWO, EU-KP7 grant inheritance and an Intramural Research Program of the $\mathrm{NIH}, \mathrm{NCl}$, Center for Cancer Research.

Financial support of the Netherlands Hearth Foundation and 'Progeria Family Circle / Stichting voor kinderen met Progeria' for the publication of this thesis is gratefully acknowledged. This thesis was further financially supported by 'Stichting Hartsvrienden RESCAR'. 


\section{CONTENTS}

$\begin{array}{lll}\text { Chapter } 1 & \text { General introduction } & 7\end{array}$

Chapter 2 Mapping of protein- and chromatin-interactions at the 15 nuclear lamina

Chapter 3 Identification of differential protein interactors of lamin A and progerin

Chapter 4 Identification of lamin A- and progerin-interacting genome regions

Chapter 5 Ageing-related chromatin defects through loss of the NURD complex

Chapter 6 Post-natal myogenic and adipogenic development defects and metabolic impairment upon loss of A-type lamins

Chapter 7 General disussion.

Summary

Samenvatting

Acknowledgements

Curriculum vitae

List of publications

Abbreviations

Colour Figures 



\section{CHAPTER 1}

General introduction 


\section{Introduction}

The nucleus is defined by the nuclear envelope (NE), which is composed of an outer and inner nuclear membrane (ONM, INM, respectively) interrupted by nuclear pore complexes (NPC) ${ }^{1}$ (Figure 1). The ONM, continuous with and functionally related to the endoplasmic reticulum $(E R)^{2}$, is separated from the distinct INM by a luminal space. The INM and ONM converge at sites of incorporated NPCs, which are homogenously spread across the NE and regulate nuclear import and export of macromolecules. Furthermore, the nucleoplasmic side of the INM is coated by the nuclear lamina, a layer of intertwined intermediate filament A-type (Lamin A, C) and B-type lamins (Lamin B1, B2), membrane proteins anchoring the lamina to the NE (including Emerin, LAP2, LBR) and proteins that modulate and interact with peripherally localized chromatin (including BAF, HP1) (Figure 1). The discovery of a mutation in the LMNA gene, that encodes for lamin $A$ and $C$, as the cause of the autosomal dominant Emery-Dreifuss muscular dystrophy ${ }^{4}$ merely a decade ago, launched numerous research studies investigating the role of A-type lamins in human disease. Since then, 11 distinct phenotypes have been associated with LMNA mutations, including several types of muscular dystrophyies lipodystrophies, cardiomyopathies, neurological disorders and premature aging syndromes. ${ }^{5}$ This broad range of LMNA related diseases, commonly referred to as laminopathies, suggests that A-type lamins play a pivotal role in diverse biological systems.

The nuclear lamina are important for maintaining nuclear integrity. Lamin fibers are assembled by head-to-tail polymerization of lamin dimers, and loss or mutation of lamin A causes dramatic structural changes that make nuclei less capable to tolerate mechanical strain and result in increased cell death. ${ }^{6-7}$ Furthermore, A-type lamins are involved in cell differentiation. Dominant negative lamin A mutants block myoblast differentiation ${ }^{8}$ and progerin, a lamin A mutant associated with the premature ageing disease HGPS (HutchinsonGilford Progeria Syndrome), inhibits adipogenisis and favors osteogenic stem cell differentiation. ${ }^{9}$ In addition, lamins regulate the expression of gene pathways by interacting with numerous transcription factors. ${ }^{10-12}$ Finally, lamins are crucial for chromatin organization as loss of A-type lamins results in thinning or loss of peripheral heterochromatin ${ }^{13}$ and various LMNA mutations modify 


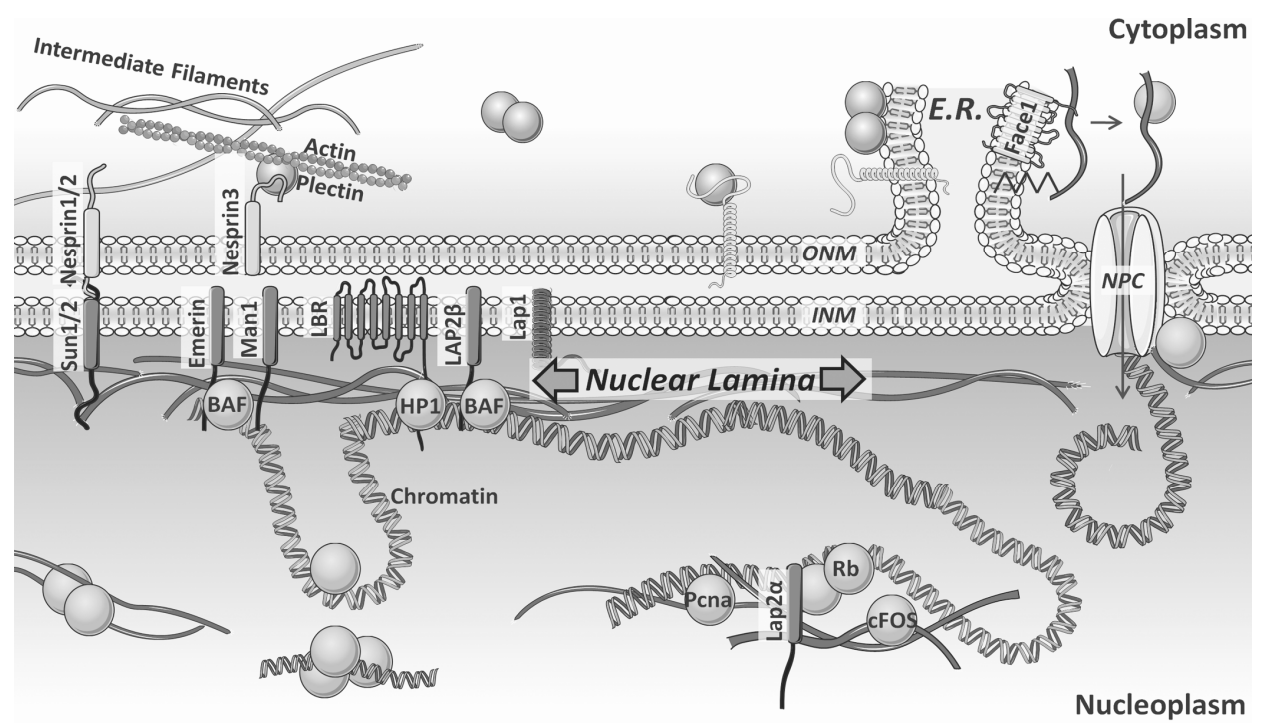

Figure 1. Schematic cartoon of various proposed interactions of lamins with inner nuclear membrane (INM) proteins, the nuclear pore complex (NPC), chromatin and various other nucleoplasmic factors. Lamins are located both underneath the INM in a thick layer, as within the nucleoplasm. Lamin A is synthesized in the endoplasmic reticulum (ER) as a precursor (prelamin A), which is farnesylated and processed by Face 1 to release mature lamin A. LAP2 $\beta$, Emerin and Man1 are localized in the INM and capable of interacting with barrier-to- autointegration factor (BAF) with their LEM domain. The lamin B receptor (LBR) interacts with lamins as well as with heterochromatic protein 1 (HP1). INM localized SUN proteins and outer nuclear membrane (ONM) localized nesprin proteins interconnect the nuclear lamina to the cytoskeletal intermediate filaments and actin fibers. Furthermore lamins interact with PCNA, and the transcription factors $\mathrm{Rb}$ and $\mathrm{cFOS}$.

chromatin structure and localization as well. ${ }^{14-15}$ Although progress has been made in understanding molecular functions of A-type lamins, many aspects still remain elusive.

The nuclear lamina are important for maintaining nuclear integrity. Lamin fibers are assembled by head-to-tail polymerization of lamin dimers, and loss or mutation of lamin A causes dramatic structural changes that make nuclei less capable to tolerate mechanical strain and result in increased cell death ${ }^{6-7}$. Furthermore, A-type lamins are involved in cell differentiation. Dominant negative lamin A mutants block myoblast differentiation ${ }^{8}$ and progerin, a lamin A mutant associated with the premature ageing disease HGPS (HutchinsonGilford Progeria Syndrome), inhibits adipogenisis and favors osteogenic stem cell differentiation ${ }^{9}$. In addition, lamins regulate the expression of gene 
pathways by interacting with numerous transcription factors ${ }^{10-12}$. Finally, lamins are crucial for chromatin organization as loss of A-type lamins results in thinning or loss of peripheral heterochromatin ${ }^{13}$ and various LMNA mutations modify chromatin structure and localization as well ${ }^{14-15}$. Although progress has been made in understanding molecular functions of A-type lamins, many aspects still remain elusive.

First, it remains uncertain whether differentiation defects are common amongst tissues affected by laminopathies. LMNA mouse models have mainly been used to study tissue defects in the young adult mice, thereby potentially missing defects in tissue differentiation, which occurs especially during early post-natal development. A common role of lamin $A$ in tissue differentiation is suggested by the observations that lamin A expression highly correlates with the level of differentiation. Lamin A expression is turned on during the embryonic developmental stage of tissue differentiation ${ }^{16-17}$ and in stem cells lamin $A$ is only expressed after differentiation induced loss of pluripotency ${ }^{18}$. Furthermore loss of lamin A expression correlates with poor histological differentiation of primary gastric carcinomas ${ }^{19}$.

A second question to be answered is how A-type lamins regulate chromatin organziation. The findings that A-type lamins interact in vitro with DNA and histones ${ }^{20-21}$ and B-type lamins bind chromatin in vivo ${ }^{22}$, led to the hypothesis that A-type lamins regulate chromatin organization by direct in vivo interaction with chromatin. Such chromatin organization could be important to regulate gene expression, as genes occupy preferred subnuclear positions in relation to their transcriptional status ${ }^{23}$. A peripheral localization of many loci is associated with a transcriptionally inactive state, and several repressed loci relocate towards the nucleus' interior upon activation ${ }^{24}$.

Finally, the major enigma in laminopathies is how mutations in one gene can cause so many phenotypic diversity. It is hypothesized that lamin A mutations affect specific lamina protein- and chromatin-interactions, thereby giving rise to laminopathy specific defects. In line with this hypothesis, Dunnigan familial partial lipodystrophy (FPLD) associated lamin A mutations specifically disrupt interaction with the adipogenic transcription factor Srebp1 ${ }^{10}$. Moreover, expression of a premature ageing associated lamin A mutant, E145K, 
mislocalizes telomeres due to erroneous lamina interaction ${ }^{15}$. The low amount of studies identifying protein or chromatin interactions specifically interrupted by LMNA mutations indicates how challenging this quest is. This is mainly due to technological restricions, as the number of applicable techniques is limited due to the insoluble nature of nuclear lamina, and those assays that can be used typically are biased or only identify a small subset of interactors. Furthermore, most assays identify general lamin interactors and do not enrich for those interactors specifically affected by LMNA mutations. These limitations prevent unbiased identification and comparison of wildtype and mutant lamin A protein and chromatin interactions in a proteome- and genome-wide fashion. Such complete mapping would help to clarify the exact function of lamin A in diverse biological processes and may provide disease mechanisms for specific laminopathies.

\section{Aim of thesis}

The overall aim of this thesis is to identify molecular mechanisms underlying laminopathies. Specifically the role of LMNA and the effect of LMNA mutations were studied in the context of disruption of protein- or chromatin interactions, chromatin organization and early postnatal tissue differentiation.

Based on this aim we defined the following goals:

- Map protein interactions of A-type lamins in a proteome-wide unbiased approach.

- Map chromatin interactions of A-type lamins in a genome-wide unbiased approach.

- Identify laminopathy-related changes in these chromatin- and proteininteractomes.

- Identify a molecular disease mechanism for a laminopathy based on these changes.

- Determine the biological relevance of A-type lamin chromatin interactions on chromatin organization and gene expression

- Determine the biological relevance of A-type lamins in tissue differentiation during early post-natal development. 
A detailed review on technological approaches to identify lamina protein and chromatin interactions is presented in Chapter 2. The identification of protein interactors of lamin A and progerin, the HGPS associated lamin A mutant, is summarized in Chapter 3. The identification of lamin A and progerin chromatin interactions and the biological relevance of these interactions in chromatin organization and gene expression is revealed in Chapter 4. Chapter 5 describes a novel disease mechanism for HGPS. The biological relevance of A-type lamins in early postnatal tissue differentiation is described in Chapter 6 . The results obtained in these studies and their implications are discussed in chapter 7. 


\section{References}

1. Foisner, R., Dynamic Connections of Nuclear Envelope Proteins to Chromatin and the Nuclear Matrix, in Nuclear Envelope Dynamics in Embryos and Somatic Cells, P. Collas, Editor. 2003.

2. Broers, J.L., C.J. Hutchison, and F.C. Ramaekers, Laminopathies. J Pathol, 2004. 204(4): p. 478-88.

3. Mattout-Drubezki, A. and Y. Gruenbaum, Dynamic interactions of nuclear lamina proteins with chromatin and transcriptional machinery. Cell. Mol. Life Sci., 2003. 60: p. 2053-2063.

4. Bonne, G., et al., Mutations in the gene encoding lamin A/C cause autosomal dominant Emery-Dreifuss muscular dystrophy. Nat Genet, 1999. 21(3): p. 285-8.

5. Broers, J.L., et al., Nuclear lamins: laminopathies and their role in premature ageing. Physiol Rev, 2006. 86(3): p. 967-1008.

6. Lammerding, J., et al., Lamin A/C deficiency causes defective nuclear mechanics and mechanotransduction. The Journal of Clinical Investigation, 2004. 113: p. 370-378.

7. Krimm, I., et al., The Ig-like Structure of the C-Terminal Domain of Lamin A/C, Mutated in Muscular Dystrophies, Cardiomyopathy, and Partial Lipodystrophy. Structure, 2002. 10: p. 811-823.

8. Mariappan, I. and V.K. Parnaik, Sequestration of pRb by cyclin D3 causes intranuclear reorganization of lamin A/C during muscle cell differentiation. Mol Biol Cell, 2005. 16(4): p. 1948-60.

9. Scaffidi, P. and T. Misteli, Lamin A-dependent misregulation of adult stem cells associated with accelerated ageing. Nat Cell Biol, 2008. 10(4): p. 452-9.

10. Lloyd, D.J., R.C. Trembath, and S. Shackleton, A novel interaction between lamin A and SREBP1: implications for parial lipodystrophy and other laminopathies. Human Molecular Genetics, 2002. 11: p. 769-777.

11. Ivorra, C., et al., A mechanism of AP-1 suppression through interaction of c-Fos with lamin A/C. Genes Dev, 2006. 20(3): p. 307-20.

12. Van Berlo, J.H., et al., A-type lamins are essential for TGF-beta1 induced PP2A to dephosphorylate transcription factors. Hum Mol Genet, 2005. 14(19): p. 2839-49.

13. Sullivan, $\mathrm{T}$., et al., Loss of A-type lamin expression compromises nuclear envelope integrity leading to muscular dystrophy. J Cell Biol, 1999. 147(5): p. 913-20.

14. Scaffidi, P. and T. Misteli, Lamin A-dependent nuclear defects in human aging. Science, 2006. 312(5776): p. 1059-63.

15. Taimen, P., et al., A progeria mutation reveals functions for lamin A in nuclear assembly, architecture, and chromosome organization. Proc Natl Acad Sci U S A, 2009.

16. Rober, R.A., et al., Cells of the cellular immune and hemopoietic system of the mouse lack lamins A/C: distinction versus other somatic cells. J Cell Sci, 1990. 95 ( Pt 4): p. 587-98.

17. Rober, R.A., K. Weber, and M. Osborn, Differential timing of nuclear lamin A/C expression in the various organs of the mouse embryo and the young animal: a developmental study. Development, 1989. 105(2): p. 365-78.

18. Constantinescu, D., et al., Lamin A/C expression is a marker of mouse and human embryonic stem cell differentiation. Stem Cells, 2006. 24(1): p. 177-85.

19. Wu, Z., et al., Reduced expression of lamin A/C correlates with poor histological differentiation and prognosis in primary gastric carcinoma. J Exp Clin Cancer Res, 2009. 28: p. 8.

20. Stierlé, V., et al., The Carboxyl-Terminal Region Common to Lamins A and C Contains a DNA Binding Domain. Biochemistry, 2003. 42: p. 4819-4828. 
14 | Chapter 1

21. Taniura, H., C. Glass, and L. Gerace, A Chromatin Binding Site in the Tail Domain of Nuclear Lamins That Interacts with Core Histones. The journal of Cell Biology, 1995. 131: p. 33-44.

22. Guelen, L., et al., Domain organization of human chromosomes revealed by mapping of nuclear lamina interactions. Nature, 2008. 453(7197): p. 948-51.

23. Takizawa, T., K.J. Meaburn, and T. Misteli, The meaning of gene positioning. Cell, 2008. 135(1): p. 9-13.

24. Zink, D., et al., Transcription-dependent spatial arrangements of CFTR and adjacent genes in human cell nuclei. J Cell Biol, 2004. 166(6): p. 815-25. 


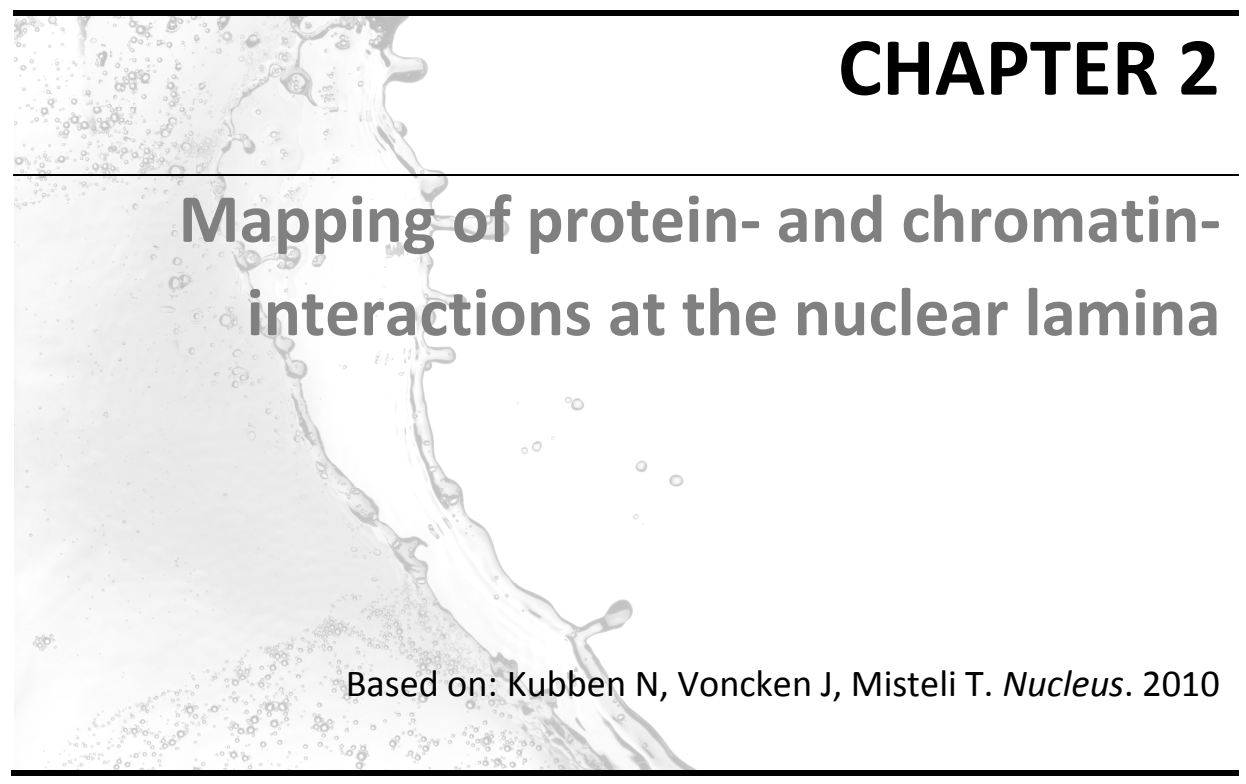




\begin{abstract}
The nuclear envelope and lamina define the nuclear periphery and are implicated in many nuclear processes, including chromatin organization, transcription and DNA replication. Mutations in lamin A proteins, major components of the lamina, interfere with these functions and cause a set of phenotypically diverse diseases referred to as laminopathies. The phenotypic diversity of laminopathies is thought to be the result of alterations in specific protein- and chromatin interactions due to lamin A mutations. Systematic identification of lamin A-protein and -chromatin interactions will be critical to uncover the molecular etiology of laminopathies. Here we summarize and critically discuss recent technology to analyze lamina protein- and chromatin interactions.
\end{abstract}




\section{Introduction}

The nuclear periphery is marked by the nuclear envelope (NE), which is composed of an outer and inner nuclear membrane (ONM, INM, respectively) interrupted by nuclear pore complexes (NPC). ${ }^{1}$ The nuclear lamina lines the $\mathrm{NE}$ and consists of a large collection of proteins, most prominently the intermediate filament A-type (Lamin A, C) and B-type lamins (Lamin B1, B2), INM proteins anchoring the lamina to the NE (including Emerin, MAN1, LAP2, Nesprin) and proteins that modulate and interact with chromatin such as BAF, HP1 and histone deacetylase 3 (Figure $1)^{2}$

Mutations in the lamina's major constituent, particularly the A-type lamins, cause a diverse set of human diseases collectively referred to as laminopathies; these include several types of muscular dystrophies, lipodystrophies, cardiomyopathies, neurological disorders and premature aging syndromes. ${ }^{3}$ The phenotypic diversity of laminopathies is hypothesized to be caused by lamin A mutations affecting specific lamina protein- and chromatin-interactions, thereby compromising nuclear integrity, higher-order chromatin organization, gene expression and/or various other nuclear processes. ${ }^{4}$ In line with this notion are the observations that Dunnigan familial partial lipodystrophy (FPLD)-associated lamin A mutations specifically disrupt interaction with the adipogenic transcription factor $S r e b p 1^{5}$ and that failure of progerin, a lamin A mutant that causes premature aging, to interact with the NURD chromatin remodeling complex contributes to loss of peripheral heterochromatin, a hallmark of the premature aging disorder Hutchinson-Gilford Progeria Syndrome (HGPS). ${ }^{6}$ Another progeria-associated lamin A mutation, E145K, leads to aberrant interaction of the lamina with telomeres. ${ }^{7}$

A key challenge in the field of lamin biology is to identify all protein and chromatin interactions at the nuclear periphery. Over the past years, several approaches have been developed and applied in order to systematically map the complete spectrum of lamina protein- and chromatin-interactions. Such approaches are crucial to elucidate the biological function of the lamina and pinpoint molecular defects for specific laminopathies. In this review we provide an overview of current technology aimed at identifying 
protein- and chromatin-interactions at the lamina. We focus on proteomeand genome-wide unbiased approaches, with particular emphasis on technical advantages and potential pitfalls in the context of subsequent mass spectrometry ${ }^{8-9}$, high-throughput microscopy and mass sequencing analysis. ${ }^{10}$ The methods discussed each with its strengths and weaknesses are all complementary but they all contribute to increasing our knowledge of function of lamins and the nuclear periphery.

\section{Protein-interactions}

\section{Visual Screens}

A basic approach to identify lamina proteins is by visual inspection of the localization of candidate proteins. In visual screens putative lamina proteins are expressed using specific tags and their localization, depending on the tag, is then detected either using live cell imaging or indirect immunofluorescence. The use of fluorescent or other epitope tags has made it possible to systematically visualize the localization of a large number of putative lamina proteins and lamin-interacting proteins by highthroughput microscopy. The most commonly used tags are GFP and Myc. The foremost advantage of visual screens is the instant registration of dynamic or abnormal changes in subcellular protein-complex localization in the context of changed cell physiology or protein mutation, respectively (Table 1). Capture of such dynamic behavior has been successfully applied ${ }^{11}$ to the study of the nuclear lamina; lamin A interactions with Rb, c-FOS and SMAD2 occur only under specified conditions, like proliferation, differentiation and TGF $\beta$ stimulation. ${ }^{12-13}$ Sensitivity, specificity and spatial resolution in cell-based imaging are somewhat confounded by the necessity to co-stain for relevant subcellular domains as well as low cellular abundance of NE proteins. ${ }^{14-17}$ Bioinformatic approaches can assist in visual screens by selecting for predicted membrane proteins ${ }^{18}$ localized at the NE. ${ }^{19}$

A powerful and more sophisticated approach of a visual screen to identify novel lamin interaction partners is the use of GFP-fusion protein libraries. In this approach 
collections of GFP-tagged fusion proteins are expressed, screened by highthroughput microscopy for subcellular localization, and clones of interest are subsequently sequenced to determine the identity of the expressed protein. Rolls et al. successfully applied a heterologous promoter-driven GFP-fusion library to screen $\mathbf{4 0 . 0 0 0}$ clones and test in an unbiased fashion whether they localized to the nuclear periphery. ${ }^{20}$ In doing so they discovered the NE membrane protein Nurim, a six-transmembrane spanning INM protein with potential isoprenylcysteine carboxymethyltransferase enzymatic activity for Caax-motifs. ${ }^{20-21} \mathrm{~A}$ drawback of protein overexpression is possible mis-localization of the fusion protein; there is an obvious need for analysis of the effect of protein expression levels on its localization and the validation of each hit by analysis of the endogenous protein. ${ }^{15,22} \mathrm{~A}$ key advantage of visual screens is that interactors which may associate with the small, but significant pool of lamin components present in the nucleoplasm, can be distinguished from those that interact with the peripheral pool of lamins. In a more physiologically relevant approach, Bickmore and colleagues identified the NE associated protein Lyric/AEG-1, an apoptosis and cell growth implicated transcriptional regulator, by using gene trapping to insert 1350 reporters into active genes. ${ }^{11,}{ }^{23}$ In this approach, endogenously expressed genes were spliced onto a genomically integrated LacZ cassette, which allowed visualization of the resulting fusion proteins by X-gal staining and $\beta$-galactosidase immunohistochemistry. ${ }^{11}$ Although such visual screens are becoming increasingly feasible, they are relatively labor intensive and slow.

\section{Biochemical Fractionation}

NE proteins are highly lipophylic and lamina proteins are strongly resistant to high concentrations of salts and detergents. These biochemical characteristics are exploited in fractionation studies to separate NE and lamina proteins from other subnuclear domains. Such isolation drastically increases the frequency of detecting NE and lamina proteins by unbiased biochemical methods e.g. mass spectrometry (MS) (Table 1). Combined with recent MS advances in complex protein mixture analysis ${ }^{9,}{ }^{24}$, such fractionation studies have the potential to contribute significantly to the identification of the full lamin proteome. As MS analysis itself cannot distinguish lamina proteins from contaminants, fraction purity is crucial. 


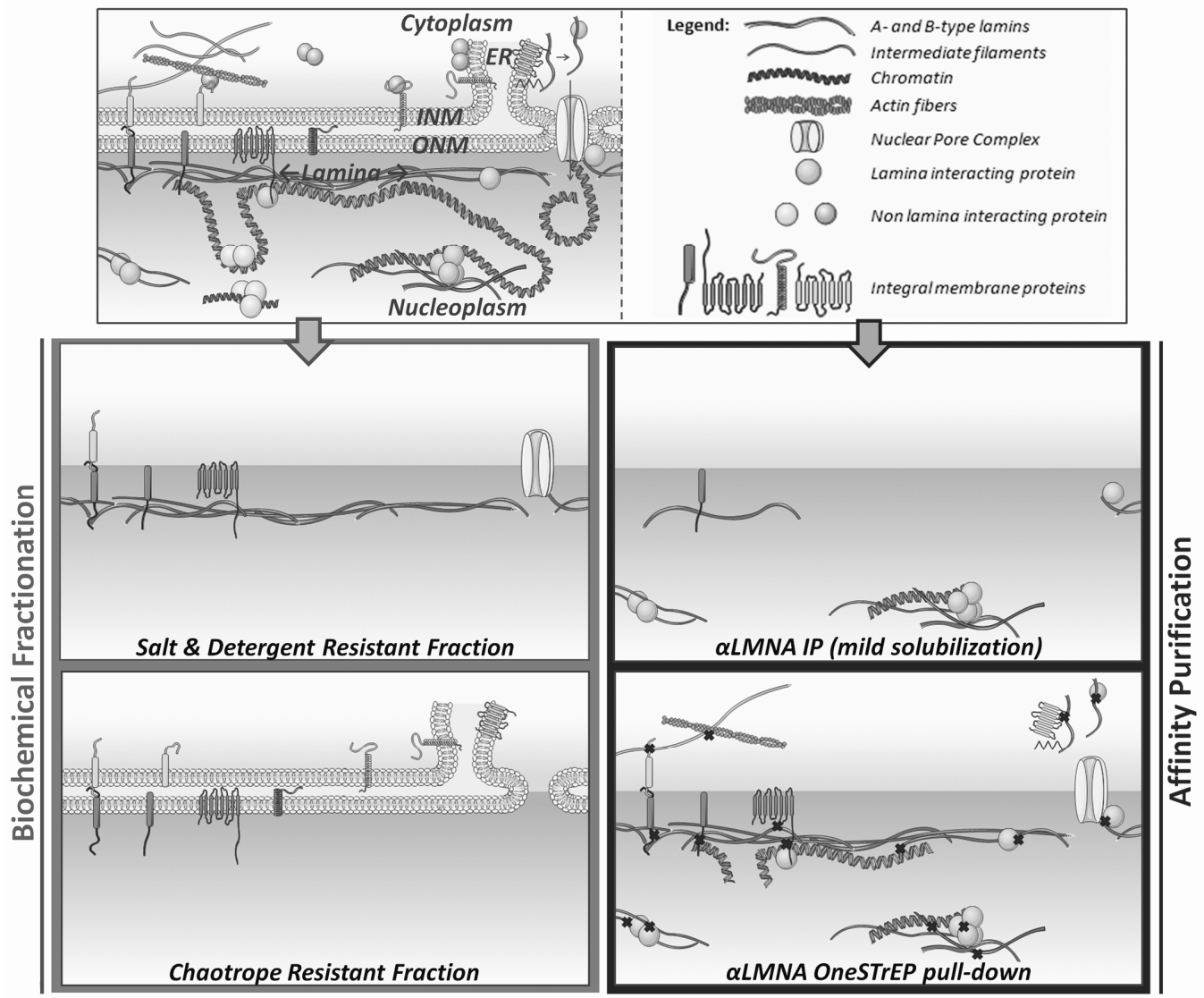

Figure 1. A schematic view of lamina and lamina-interacting protein fractions purified by various techniques. The nuclear periphery consists of an inner nuclear membrane (INM), outer nuclear membrane (ONM), and is connected to the endoplasmic reticulum (ER). Salt solubilizes (left) weakly attached lamina proteins, but not the nuclear lamina. Detergents preferentially dissolve membrane proteins that are not anchored in the detergent resistant lamina. ${ }^{28,66}$ Chaotropes and alkaline extraction generate an insoluble fraction mainly consisting of integral membrane proteins. ${ }^{66}$ Immunoprecipitations (right) with an antibody directed against lamin A/C, using mild lysis conditions (for example 01\% NP-40, $250 \mathrm{mM} \mathrm{NaCl}{ }^{12}$ ), preferentially dissolve and precipitate nucleosoluble A-type lamins and protein interactors. ${ }^{35}$ For a lamin A OST pull-down assay ${ }^{46}$, cross-linking, indicated by crosses, captures proteinprotein and protein-chromatin interactions, and allows solubilization of the total lamin $A / C$ pool while preserving interactions. 
Various assays have been developed to fractionate NE and lamina proteome subsets, each with a specific trade-off between obtained purity and amount of background proteins.

Protein Correlation Profiling (PCP) was developed to determine subcellular protein localization in crude extracts, separated by rate-zonal centrifugation into fractions which are subsequently analyzed by MS analysis (Table 1). ${ }^{25}$ In essence, the technology relies on co-detection of proteins known to reside in the organelle of interest and novel proteins. ${ }^{25}$ The main advantage of PCP is its ability to detect multiple co-segregating proteins in a complex mixture without the need to fully isolate and highly purify subcellular fractions. However, PCP comes at a price: as many subcellular domains are only partially separated by centrifugation, non-specific interactors co-purify and separation of true interactors relies strongly on computational analysis. Follow-up studies to characterize the properties of identified proteins are imperative. Although PCP analysis has not been applied yet to distinguish and define nuclear lamina/envelope proteins domains, the fact that differences in rate-zonal resolving properties have previously been used to purify NE fractions ${ }^{26}$, makes PCP a promising technique to identify NE and lamina proteins.

In comparison to PCP, differential extraction assays use more highly purified fractions for MS analysis and consequently reduce non-specific copurification (Table 1). In general, biochemical fractionation of the NE starts with isolation of nuclei, removal of non-NE membrane fractions by centrifugation and digestion of chromatin to remove nucleoplasmic contents. Crude NE and lamina fractions are subsequently extracted in salt, detergent, and chaotrope or alkaline buffers to further remove different types of proteins. Salt preferentially dissolves chromatin-bound proteins and other non-membrane, weakly attached lamina proteins, but not lamina proteins (Figure 1). Detergents (Octyl glucoside, Trx-100, Empigen BB) mimic the lipid bilayer environment and particularly dissolve membraneassociated proteins, except those that are anchored to the detergent resistant lamina (Figure 1). In this manner Cronshaw et al. successfully identified 6 novel NPC components, among which ALADIN, the gene mutated in the triple-A or Allgrove syndrome ${ }^{27}$ Chaotropes (urea, thiourea) and alkalines $(\mathrm{NaOH})$ are used to solubilize cytoskeletal, chromatin and lamina components, while leaving integral transmembrane proteins 
embedded in the insoluble membrane fraction (Figure 1). As such lamina and lamina anchored INM/NE proteins are extracted by combined application of salt and detergent (Figure $1 ; 29-30$ ), whereas integral INMONM- and ER-membrane proteins are purified by chaotrope or alkaline extraction (Figure 1).

Despite increased sample purity by applying differential extractions, these fractionation studies are still hampered by co-purification of non-specific interactors, in particular from the peripheral ER, which is continuous with the ONM and therefore difficult to separate from the NE and lamina. To further reduce false positive hits, Dreger et al. compared salt, detergent and chaotrope/alkaline extractions, and were able to screen out ER contaminants in chaotrope/alkaline resistant fractions. ${ }^{28}$ This strategy identified 19 previously unknown and putative integral INM proteins, including Unc84a (Sun1), LUMA and two LAP2 isoforms. ${ }^{28} \mathrm{~A}$ disadvantage of this comparative approach is that selection is based on the assumption that all INM and lamina proteins have similar biochemical extraction characteristics. However, several lamina anchored INM proteins, like emerin, LBR and LAP1, behave biochemically very different in detergent or chaotrope-based extraction. ${ }^{28}$

In an extenstion of purification methods, subtractive approaches can be used to filter out ER residing proteins. In these methods proteins identified in non-NE/lamina fractions, thus enriched for background, are subtracted from proteins detected in differential NE/lamina extracts. Schirmer and colleagues used microsomal membrane fractions as a source of background proteins, as these ER-rich fractions are easily obtained and can be prepared free of nuclear membranes. ${ }^{29}$ By combining differential fractionation and subtractive proteomics, they identified 67 previously unidentified NE transmembrane proteins. The disadvantage of using a reference background source is that proteins that reside in both the ER and NE, like AEG-1/Lyric, Sec13 and Torsin A, are inadvertently discarded. ${ }^{30-32}$ This is a serious concern, since it is now estimated that one third of cellular proteins have multiple organellar localizations. ${ }^{25}$ 


\section{Affinity purification}

An alternative approach to identifying lamina interactions is affinity purification. In these approaches a protein of interest is epitope tagged and the bait protein is affinity purified using antibodies against the tag (Table 1). Affinity purification can identify and distinguish bound protein complexes from each other by co-elution and MS analysis. Such gradual elution was used for example to separate emerin-interacting RNA processing, signaling and chromatin remodeling complexes. ${ }^{33}$ In addition, it is possible to study interactions in the context of posttranslational modifications by using specific antibodies directed against them, for example phosphorylationdependent LBR-p32/p34 ${ }^{34}$, lamin A-Rb and $-S \operatorname{Smad2} 2^{12}$ interactions. In contrast to biochemical fractionation, in which fractions are generated under denaturing conditions, the main challenge in affinity purification assays is to extract as many NE and lamina proteins while leaving protein interactions intact. Overly stringent solubilization dissolves many proteins at the cost of disrupting complexes, while excessively mild conditions do not dissolve all relevant interactors. Various strategies have been applied to find a good balance for this trade-off.

In classic immunoprecipitations (IPs) solubilization conditions are optimized for the protein of interest (Table 1). Low amounts of detergents and salt preferentially solubilize nucleoplasmic pools of proteins (Figure 1), as described to exist for lamin $A^{35}$, and were mainly applied to study easy extractable, weakly bound NE interactors (Smad2, PP2A, Rb, Ubc9, hnRNP1, EGF1, SREBP1; See Table 2) ${ }^{5,12,36-38}$. Increasing amounts of detergents, salts and the solvent glycerol ${ }^{39}$ successfully solubilize protein complexes of wellanchored NE and lamina components (LAP2 $\beta$, Emerin, Nesprin2, Lamin B; Table 2), although sometimes at the cost of disrupting interactions $(\text { LaminA/B1/B2 - LAP2 } \beta)^{40}$ (Table 2). Highly stringent conditions were applied when studying NPC proteins as they were assumed to be highly stable structures (Table 2). ${ }^{41-43}$ Even though NPCs apparently better withstand stringent extraction, increased stringency of washing buffers disrupts interactions (Table 2). ${ }^{41}$ An additional disadvantage to be accounted for is that lysis buffers also can affect the antibody/epitopeinteraction. Various groups therefore prefer to dilute buffer compositions after initial lysis, which combines increased solubilization with the ability of protein complexes to reassemble and antibodies to bind under sequential 
milder conditions. This strategy was used to identify unknown interactors for BAF and Emerin (Table 2). ${ }^{33,44}$ Other limitations of antibody-based methods include the unavailability of IP-suited antibodies ${ }^{36}$, antibodies that recognize multiple epitopes (MAN1 antiserum) ${ }^{36}$, antibodies that cross-react undesirably with non-mature forms of a protein (prelamin $A$ versus lamin $A)^{38}$ or even disrupt protein interactions (laminA/B2 - LAP2 $\beta$ ). ${ }^{40}$

To avoid the use of antibodies, precipitations can be performed using bacterially expressed and purified baits, conjugated to beads prior to incubation with solubilized protein extracts. Fusing the bait to an epitope tag contributes to high quality purification of the bait, and efficient precipitation of interactors from protein extracts (Table 1). This approach was combined with mild lysis, for BAF and emerin interactors ${ }^{33}, 44$, or more stringent buffers for lamin-LAP2 $\beta$ and -nesprin 2 interactions (Table 2). ${ }^{40,45}$ In accordance with mild solubilization, identified BAF and emerin interactors represented many proteins that also reside outside the nuclear periphery (PARP, HP1gamma, RBBP4,7). ${ }^{40,45}$ Another advantage of using a bait is the ability to pinpoint interactions to relevant protein domains, as the bait does not have to be incorporated in vivo and therefore cannot mislocalize, as described to occur for LAP2 $\beta$ constructs. ${ }^{40}$ The main disadvantages of IPS using a bait is that solubilization issues still remain and interactions formed in vitro do not necessarily occur in vivo.

A major step forward in overcoming solubilization problems is the OneSTrEP (OST) pull-down assay ${ }^{6,46}$, which combines the use of a biotin resembling OST-tag ${ }^{47-48}$ with mild cross-linking of cells prior to solubilization (Table 1). Cross-linking allows extraction of the total lamin A/C pool ${ }^{46}$ (Figure 1 ) while leaving protein interactions intact. The OST-tagged protein and its interactors can then be highly efficiently precipitated under denaturing conditions using a high-affinity, engineered streptavidin analogue (Table 2). Stringent washes reduce background, especially relevant for A-type lamins, known to be "sticky" proteins and reported to precipitate in negative pulldown controls as well. ${ }^{44}$ The main advantages of the OST pull-down are that it can be used to identify a full in vivo interactome of a protein regardless of its subnuclear position, detect the effect mutations have on these interactions and identify weak interactors. OST pull-downs have been used to compare protein interactions for lamin A and progerin, an HGPS causing 
lamin A mutant, and detected a decreased interaction for progerin with NPC components. ${ }^{44}$ The main disadvantages of this approach is that it is not possible to study endogenous proteins and that cross-linking does not allow gradual elution and thereby separation of interacting protein complexes (Table 1).

\section{Yeast Two-Hybrid}

An interesting alternative to affinity purifications is yeast two-hybrid ( $\mathrm{Y} 2 \mathrm{H})$ (Table 1), in which a direct interaction between a DNA-binding domain fused bait and a co-expressed transcriptional activating domain fused prey allows growth under restrictive conditions. When the primary interest is to identify weak and direct interactors $\mathrm{Y} 2 \mathrm{H}$ is useful as bait and prey are expressed by strong exogenous promoters, protein solubilization is not required and weak interactions are sufficient to allow restrictive growth. The focus on direct protein interactions, which might best reflect the core activities of the protein of interest, restricts the mapping of a complete interactome. Protein fragments can easily be used as bait since they don't have to be incorporated in vivo, and have been applied to describe interactions between the specific domains of the nuclear envelope proteins Otefin, Lamin A, Nesprin2 and. ${ }^{45,49-50}$ The benefit of choosing the exact bait composition can further be exploited by choosing domains involved in disease mechanisms, like the 50 amino acid deleted region in progerin, shown in a $\mathrm{Y} 2 \mathrm{H}$ screen to interact with the NURD chromatin remodeling complex component Rbbp4. ${ }^{6}$ Disadvantages of $\mathrm{Y} 2 \mathrm{H}$ assays are the lack of information on protein complex composition, the inability to study posttranslational modifications and the large amount of false positives identified. The large amount of background can be caused by endogenous transcriptional activity of bait or prey proteins, as reported for cFOS domains used to map lamin $A$ interaction ${ }^{13}$, bait or prey proteins affecting yeast growth under restrictive conditions, and the fact that investigated interactions may never occur in vivo (Table 1). In addition, $\mathrm{Y} 2 \mathrm{H}$ approaches for lamin proteins are particularly difficult since expression of lamin-fusion proteins in S. Cerevisiae has detrimental effects on the organism. 


\section{Chromatin-interactions}

In addition to protein-protein interactions, the importance of interactions between chromatin and the lamina is increasingly appreciated. In particular, many lamin proteins are now known to directly or indirectly interact with chromatin and chromatin defects are a hallmark of several laminopathies. ${ }^{51-}$

53 These observations have catalyzed the development of unbiased screening techniques for chromatin interactions at the NE. A broad distinction can be made between assays using affinity purification and those based on enzymatic activity (Figure 2, Table 3).

\section{Affinity based approaches: ChIP \& OST pull-down}

Chromatin-protein interactions are most commonly interrogated using chromatin-immunoprecipitation (ChIP) methods. In this approach, a protein of interest is cross-linked to chromatin and immunoprecipitated using a specific antibody against the protein. The DNA is then identified either by targeted PCR methods or by genome-wide microarray or sequencing approaches. The major difference between conventional IPs and ChIP is the addition of a cross-linking step prior to solubilization of intact proteinchromatin complexes. Cross-linking provides the advantage of combining ultra-sonication and stringent lysis, to shear DNA and dissolve NE proteins (Figure 2), with good preservation of protein-chromatin interactions (Table 3). Just as for classic IPs, lysis buffers still need to be attuned to the strength of the epitope-antigen interaction. For this reason, initial ChIP studies were performed on Myc-tagged NPC proteins in S. Cerevisae ${ }^{54}$, as NPCs are easily dissolved in the absence of nuclear lamina and high quality ChIP-suited Myc antibodies are commercially available. For the INM protein Src1, a MAN1 resembling protein, interactions with (sub)telomeric regions were identified in yeast using a high affinity protA-system. ${ }^{55-56}$ Silver et al. used endogenous Nup93 in HeLa cells by dialyzing the initial lysis buffer to a milder variant prior to incubation with antibodies. ${ }^{57}$ The foremost advantage of using antibodies is the ability to study endogenous proteins and chromatin interactions in the context of posttranslational modifications (Table 3).

A modification of the classical ChIP approach is the use of the OneSTrEP tag (OST) pull-down which enables high affinity precipitation of OST-tagged proteins under denaturing conditions completely dissolving A-type lamins, 


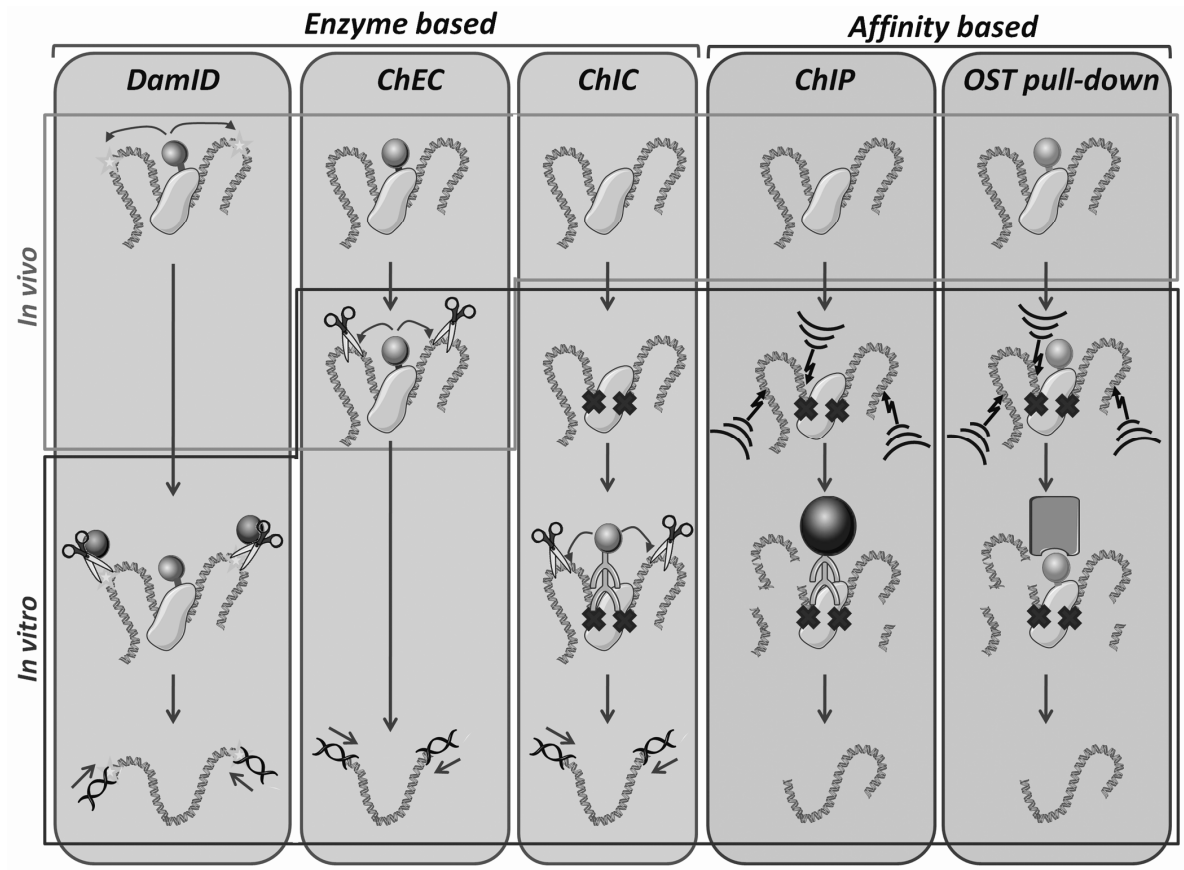

Figure 2. Schematic overview of techniques to identify chromatin interactions, which are categorized in enzymatic- and affinity-based approaches. For DamID ${ }^{59}$ a DNA adenine methyltransferase (Dam) tag (ball on stick) is fused to the protein of interest and adenylates (star) bound chromatin in vivo, enabling in vitro selective Dpnl (scissor) restriction and subsequent amplification of restricted chromatin by ligation mediated PCR (LMPCR). For in vivo chromatin endogenous cleavage $(\mathrm{ChEC})^{64}$ a protein of interest is fused to a micrococcal nuclease (MNase) tag, which introduces DNA double strand breaks (scissors) upon introduction of calcium chloride to weakly permeabilized cells. Due to the mild permeabilization of cells prior to addition of calcium chloride for activation, the MNase digestion step is indicated as being partially in vitro and in vivo. Restricted DNA is amplified by LMPCR. For chromatin immunocleavage $(\mathrm{ChIC})^{64}$ cells are cross-linked (crosses). In vitro, MNase-conjugated antibody interacts with the epitope of interest and induces DNA breaks enabling LMPCR amplification of cleaved chromatin. For chromatin immunoprecipitation (ChIP) chromatin-protein interactions are cross-linked and chromatin is randomly sheared, typically by ultrasonication, ( lightning arrow and stripes). Antibodies are used to precipitate the endogenous protein of interest with the help of antibody binding beads (big ball). In a OneSTrEP (OST) pull-down a OST-tagged protein is expressed. ${ }^{58}$ Cells are cross-linked and ultrasonicated. The OST-protein is highly efficiently precipitated by a streptactin matrix (big square). 
comparable to the use of OST tags used for pull-down of proteins (Table 3). ${ }^{46,58}$ The OST pull-down for identification of chromatin interactions is highly similar to that for detecting protein interactions, and only includes slight changes in sonication and washing conditions. ${ }^{46,58}$ Although OST pull-downs have the advantage of easy solubilization and high affinity pull-down without the use of antibodies, which in the case of lamin A have not been ideal in ChIP experiments, a limitation is the inability to directly study endogenous proteins and posttranslational modifications (Table 3 ).

\section{Enzymatic activity based approaches: DamID, In vivo ChEC, ChIC}

DamID is an enzyme-based method for the in vivo mapping of chromatinprotein interactions. In DamID a protein of interest is fused to a DNA adenine methyltransferase (Dam) and expressed. Upon binding of the fusion protein to chromatin, the Dam activity marks in the vicinity bound chromatin by methylation, thereby enabling selective Dpnl restriction in vitro. The marked sites can then be identified by targeted PCR or, more commonly, by genome-wide microarray analysis and deep-sequencing ${ }^{59}$ (Figure 2). The main advantage of using a tag that enzymatically marks DNA is that only isolation of DNA, not of intact protein/chromatin complexes, is required, thus eliminating any issues related to interaction stability. In addition, there is no need for cross-linking, thereby avoiding potential fixation artifacts. These characteristics made DamID the first technique to characterize and compare chromatin interactions for the relative insoluble lamin B and emerin proteins in a genome-wide fashion and resulted in the characterization of lamin associated domains (LADs) which define regions of the genome that preferentially interact with the lamina. ${ }^{59-60}$ Disadvantages of DamID include the inability to study posttranslational modifications, a slightly reduced resolution

compared to alternative assays and potential interference of the tag with protein localization or functioning (Table 3 ) ${ }^{61}$ In addition, since DamID relies on the expression of an enzymatically active fusion protein, it monitors chromatin interactions over a relative long period of time (app. 24 hours) and therefore is less useful to detect rapid interaction changes and dynamic reorganization of chromatin. Due to the DNA binding activities and high enzymatic activity of Dam, tagged proteins can only be expressed in trace 
amounts in order to prevent saturation of non-targeted DNA methylation. ${ }^{62}$ This makes it not possible to study chromatin interactions in a dosage dependent manner, which could be relevant for diseases in which phenotypes are dependent on the amount of protein, such as HGPS. ${ }^{63}$

An alternative method to measure protein-chromatin interactions is ChEC (chromatin endogenous cleavage). In this approach, micrococcal nuclease (MNase) is fused to a protein of interest and expressed. The fusion protein is recruited to its endogenous sites on chromatin where the MNase introduces double strand breaks at nuclease hypersensitive sites (HS) ${ }^{64-65}$ The MNase tag remains inactive under physiological $\mathrm{Ca}^{2+}$ concentrations, which provides the ability to selectively turn its activity on in vivo by addition of calcium chloride to mildly permeabilized cells. Cleaved chromatin can either be directly used to map HS sites by indirect endlabeling and Southern blotting, or is first selectively amplified by ligationmediated PCR prior to genome-wide microarray analysis and deepsequencing (Figure 2). ${ }^{64}$ Laemmli and colleagues used this approach to map chromatin interactions of the nuclear pore complex protein Nup2 and found that Nup2-gene promoter interactions typically are an early event of gene activation and are independent of transcription. ${ }^{64}$ Control over MNase activity and relative short times needed for

digestion make this assay suitable for detection of rapid changes in interactions. The major strength of in vivo ChEC is that there is no need to dissolve intact protein/chromatin complexes and information on chromatin structure is obtained by mapping HS (Table 3 ). In comparison to DamID higher expression levels of MNase-tagged proteins can be used, although at very high expression levels background issues were reported. ${ }^{65}$ ChEC can also be modified to study posttranslational modifications as the MNase tag can also be conjugated to an antibody of interest. This in vitro method is referred to as chromatin immunocleavage (ChIC). In ChIC cross-linked cells are lysed and incubated with MNase-coupled antibodies that bind to the epitope of interest after induction of DNA cleavage by $\mathrm{Ca}^{2+}$ (Figure 2). ChIC is a hybrid between affinity- and enzyme-based approaches in that it uses cross-linking and antibodies, but does not need to fully dissolve and precipitate intact protein-chromatin complexes due to the use of enzymatic activity, which specifically marks bound DNA (Table 3). 


\section{Concluding remarks}

The characterization of structural and other functional components in the nuclear lamina is vital for our understanding of higher-order chromatin organization, transcription, DNA replication and various other nuclear processes. Recent development of powerful techniques to map protein and chromatin interactions have begun to reveal these roles.

Several approaches to identify the interaction network at the nuclear periphery are now available. These methods are all complementary and each has its own usefulness and limitations. Ideally, one would map the interactions of proteins and chromatin using multiple, complementary techniques. At present this is practically often not feasible, however, as interaction-detection methods are improved, it should become possible to interrogate interactions by multiple means. For now, the choice of method often relies on the particular question to be addressed. When it is important to identify multiple sub-cellular localizations of a protein, visual screens are the best option. Biochemical fractionation studies best assist in revealing a full proteome. A more detailed impression of an individual protein's interactome can be obtained by classic IPs to study endogenous proteins, by OST pull-down to identify weak and relative insoluble proteins, and by IPs using a bait to distinguish individual protein complexes. Mapping interactions of direct and weak interactors to protein domains can best be done by $\mathrm{Y} 2 \mathrm{H}$. For DNA interactions a careful choice has to be made between the need for studying endogenous proteins and posttranslational modifications (ChIP, ChIC), obtaining extra information on chromatin structure (In vivo $\mathrm{ChEC}, \mathrm{ChIC}$ ), full protein solubilization and obtaining an instant snapshot of interactions (OST pull-down), or not dissolving protein/chromatin complexes and capturing interactions over a longer period of time (DamID).

Even though the overlap between various chromatin techniques is slightly bigger then for protein techniques, in both fields a combinatorial or comparative use of techniques, as well as the target proteins they are applied on, will lead to more reliable results and provide a better understanding of the NE. These methods are becoming increasingly routinely used in many laboratories and is no doubt that proteome and 
genome-wide mapping method and screening for mutation-induced interaction changes will play a key role in unraveling nuclear lamina function and laminopathy disease mechanism. 
Tables

Table 1: Techniques to identify protein interactions at the nuclear lamina

\begin{tabular}{|c|c|c|c|c|c|c|c|c|c|c|c|}
\hline \multirow{2}{*}{ 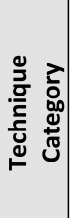 } & \multirow[b]{2}{*}{ Technique } & \multirow{2}{*}{ 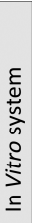 } & \multirow[b]{2}{*}{ 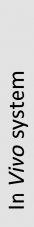 } & \multirow{2}{*}{ 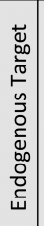 } & \multirow{2}{*}{ 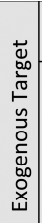 } & \multicolumn{4}{|c|}{$\begin{array}{c}\text { Interactors } \\
\text { bind }\end{array}$} & \multirow{2}{*}{ 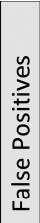 } & \multirow[b]{2}{*}{ Advantages } \\
\hline & & & & & & 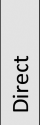 & 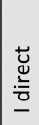 & 吕 & $\sum_{3}^{2}$ & & \\
\hline 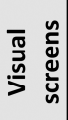 & $\begin{array}{l}\text { Visualize } \\
\text { tagged } \\
\text { proteins }\end{array}$ & - & + & \pm & + & + & + & + & + & - & $\begin{array}{l}\text { - No fractionation of NE compartment required } \\
\text { - Possible to identify multiple cellular locations } \\
\text { - Possible to screen for stimuli influencing subcellular localization }\end{array}$ \\
\hline \multirow{2}{*}{ 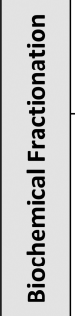 } & $\begin{array}{l}\text { Protein } \\
\text { Correlation } \\
\text { profiling* }\end{array}$ & - & + & + & - & + & + & + & \pm & + & $\begin{array}{l}\text { - Applicable on crude extracts, thus useful for organelles difficult to } \\
\text { purify } \\
\text { - Copurification provides information about protein complex composition }\end{array}$ \\
\hline & $\begin{array}{l}\text { Differential } \\
\text { Extractions }\end{array}$ & - & + & + & - & + & + & + & - & \pm & $\begin{array}{l}\text { - Specifically enrich for NPC, lamina or integral INM proteins } \\
\text { - Ability to detect a large part of the complete NE proteome } \\
\text { - No organelle specific markers necessary }\end{array}$ \\
\hline \multirow{3}{*}{ 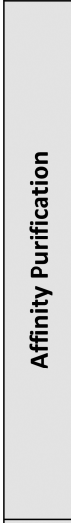 } & $\begin{array}{l}\text { Classic } \\
\text { Immuno- } \\
\text { precipitation }\end{array}$ & - & + & + & + & + & + & + & - & \pm & $\begin{array}{l}\text { - Specifically enrich for interactors of protein of interest } \\
\text { - Possibility to gradually elute interactors, which provides information } \\
\text { on protein complex composition } \\
\text { - Influence of mutations on protein interactions can be determined } \\
\text { - IP possible for specific posttranslational modifications }\end{array}$ \\
\hline & $\begin{array}{l}\text { Immuno- } \\
\text { precipitation } \\
\text { with bait }\end{array}$ & - & + & - & + & + & + & + & - & \pm & $\begin{array}{l}\text { - Specifically enrich for interactors of protein of interest } \\
\text { - Possibility to gradually elute interactors, which provides information } \\
\text { on protein complex composition } \\
\text { - Efficient and specific precipitation, because bait is translated in vitro } \\
\text { - Influence of mutations on protein interactions can be determined }\end{array}$ \\
\hline & $\begin{array}{l}\text { OneSTrEP } \\
\text { Pulldown }\end{array}$ & - & + & - & + & + & + & + & + & \pm & $\begin{array}{l}\text { - Specifically enrich for interactors of protein of interest } \\
\text { - Protein complexes can be fully solubilized intact } \\
\text { - AB-free high affinity purification } \\
\text { - Influence of mutations on protein interactions can be easily determined } \\
\text { - Interactions dependent on chromatin, as well as weakly attached NE } \\
\text { proteins detected. }\end{array}$ \\
\hline 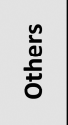 & $\begin{array}{c}\text { Yeast Two- } \\
\text { Hybrid }\end{array}$ & - & + & - & + & + & - & + & + & + & $\begin{array}{l}\text { - Interactions can be easily mapped to protein domains } \\
\text { - Influence of mutations on interactions can be easily determined } \\
\text { - Tissue specific libraries can be used to identify tissue specific } \\
\text { interactors }\end{array}$ \\
\hline
\end{tabular}

Legend: No (-), endogenously expressed, but genetically modified ( \pm ), Yes $(+)$. For 'false positives'-column: low $(-)$, moderate $( \pm)$, high $(+) ; A B=A n t i b o d y ;{ }^{*}$ Data not analyzed for subnuclear structures yet. 


\begin{tabular}{|c|c|}
\hline Disadvantages & References \\
\hline $\begin{array}{l}\text { - No enrichment for NE proteins } \\
\text { - Co-staining for organelle specific markers necessary } \\
\text { - Weakly expressed endogenous proteins potentially not visualized } \\
\text { - Protein overexpression can result in mislocalization } \\
\text { - Tag can influence protein localization }\end{array}$ & $\begin{array}{c}(11,14-16,20, \\
22,67)\end{array}$ \\
\hline $\begin{array}{l}\text { - No clear cut off values for different organelle fractions } \\
\text { - Organelle specific markers necessary } \\
\text { - Difficult to compare different samples } \\
\text { - Influence of mutations on protein interactions difficult to determine }\end{array}$ & (25) \\
\hline $\begin{array}{l}\text { - Background due to co-isolation of non-NE membranal fractions. } \\
\text { - No information about protein interactions and complexes composition } \\
\text { provided } \\
\text { - Needs optimization per tissue type } \\
\text { - Interactions dependent on chromatin, as well as weakly attached NE } \\
\text { proteins less well detected. } \\
\text { - Influence of mutations on protein interactions difficult to determine }\end{array}$ & $(27-28,68-70)$ \\
\hline $\begin{array}{l}\text { - Difficult to fully solubilize intact protein complexes } \\
\text { - No direct information provided on (multiple) localization(s) protein of } \\
\text { interest } \\
\text { - Quality highly dependent on } A B\end{array}$ & $\begin{array}{l}(5,12-13,33 \\
36,38,40-45 \\
62,71-74)\end{array}$ \\
\hline $\begin{array}{l}\text { - Difficult to fully solubilize intact protein complexes } \\
\text { - No direct information provided on (multiple) localization(s) protein of } \\
\text { interest } \\
\text { - Quality highly dependent on AB } \\
\text { - IP not possible for specific posttranslational modifications } \\
\text { - Protein interactions are possible not native }\end{array}$ & $\begin{array}{c}(33,40,44-45 \\
72)\end{array}$ \\
\hline $\begin{array}{l}\text { - No direct information provided on (multiple) localization(s) protein of } \\
\text { interest } \\
\text { - Gradual elution of interactors not possible due to cross-linking. } \\
\text { Therefore no extra information on protein complex composition } \\
\text { - Pulldown not possible for specific posttranslational modifications }\end{array}$ & $(6,46)$ \\
\hline $\begin{array}{l}\text { - Found interactions do not necessarily occur in vivo } \\
\text { - No information provided on protein complex composition or subcellular } \\
\text { localization } \\
\text { - Inability to study posttranslational modifications }\end{array}$ & $\begin{array}{c}(6,13,37,45 \\
50,72,75)\end{array}$ \\
\hline
\end{tabular}


Table 2: Solubilization conditions to identify protein interactions at the nuclear lamina

\begin{tabular}{|c|c|c|c|c|c|c|c|c|c|c|c|c|c|c|}
\hline \multirow{3}{*}{ 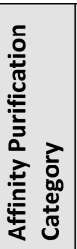 } & \multirow[b]{3}{*}{ Interactions } & \multirow{3}{*}{ 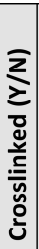 } & \multirow{3}{*}{ 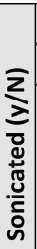 } & \multicolumn{10}{|c|}{ Lysis buffer } & \multirow[b]{3}{*}{ 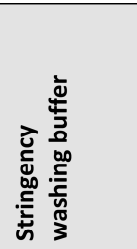 } \\
\hline & & & & \multicolumn{4}{|c|}{ Detergents } & \multicolumn{2}{|c|}{ Salts } & \multicolumn{4}{|c|}{ Others } & \\
\hline & & & & $\frac{\bar{o}}{\tilde{n}}$ & $\begin{array}{l}\text { হ } \\
\text { o } \\
\text { d } \\
\frac{1}{2}\end{array}$ & 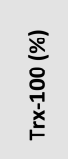 & 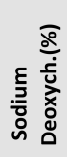 & $\begin{array}{l}\sum_{\underline{E}} \\
\overline{\bar{J}} \\
\text { z }\end{array}$ & $\begin{array}{l}\underset{\sum}{\underline{\xi}} \\
\overline{\bar{y}}\end{array}$ & $\begin{array}{l}\frac{\overline{0}}{\overline{0}} \\
\frac{0}{0} \\
\frac{\bar{v}}{0}\end{array}$ & $\frac{\sum_{E}^{E}}{E}$ & 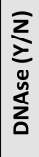 & 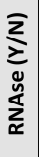 & \\
\hline \multirow{12}{*}{ 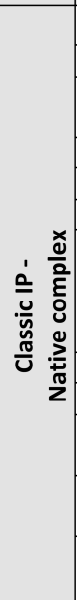 } & Man1/SMAD2 & $\mathrm{N}$ & $Y$ & & 0.1 & & & & & & 1 & $\mathrm{~N}$ & $\mathrm{~N}$ & Similar \\
\hline & LMNA\&C/SMAD2/ PP2A/Rb & $\mathrm{N}$ & $\mathrm{Y}$ & & 0.1 & & & 250 & & & & $\mathrm{~N}$ & $\mathrm{~N}$ & Similar \\
\hline & $\begin{array}{l}\text { LMNA\&C/Progerin/Ubc9/hnR } \\
\text { NPE1/EGF1 }\end{array}$ & $N$ & $\mathrm{~N}$ & & & 1.0 & & & & & & $\mathrm{~N}$ & $\mathrm{~N}$ & Similar \\
\hline & LMNA/Sun1 & $\mathrm{N}$ & $\mathrm{N}$ & & & 1.0 & & 50 & & & & $\mathrm{~N}$ & $\mathrm{~N}$ & Similar \\
\hline & preLMNA/ SREBP1 & $\mathrm{N}$ & $\mathrm{N}$ & & 1.0 & & & 100 & & & & $\mathrm{~N}$ & $\mathrm{~N}$ & Similar \\
\hline & LMNA/SREBP1 & $\mathrm{N}$ & $\mathrm{N}$ & & & 1.0 & & 150 & & & & $\mathrm{~N}$ & $\mathrm{~N}$ & Similar \\
\hline & LAP2B/LMNA/LMNB1/LMNB2 & $\mathrm{N}$ & $\mathrm{N}$ & & & 1.0 & & 150 & & & & $\mathrm{~N}$ & $\mathrm{~N}$ & $\begin{array}{c}150-300 \mathrm{mM} \\
\mathrm{NaCl}\end{array}$ \\
\hline & LMNA/cFOS & $\mathrm{N}$ & $\mathrm{N}$ & & 1.0 & & & 150 & & 10 & & & & $500 \mathrm{mM} \mathrm{NaCl}$ \\
\hline & Emerin/Nesprin2 & $\mathrm{N}$ & $\mathrm{N}$ & & & 1.0 & 0.50 & 150 & & & & $\mathrm{~N}$ & $\mathrm{~N}$ & Similar \\
\hline & Nup153/p250/p62 & $\mathrm{N}$ & $\mathrm{N}$ & & & 0.5 & & 500 & & & 1 & $\mathrm{~N}$ & $\mathrm{~N}$ & $\begin{array}{l}0-0.1 \\
\% \text { SDS }\end{array}$ \\
\hline & Nup50/MC2R & $N$ & $N$ & & $0.1^{*}$ & & & 420 & $\begin{array}{c}1500 \\
*\end{array}$ & 25 & 0.5 & $N$ & $\mathrm{~N}$ & Similar \\
\hline & Nup96,107,120,133 & $\mathrm{N}$ & $\mathrm{N}$ & & & 2.0 & & & 150 & & 1 & $Y$ & Y & Similar \\
\hline \multirow{4}{*}{ 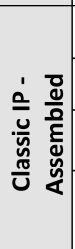 } & BAF/Various Interactors & $\mathrm{N}$ & $N$ & & & $\begin{array}{c}0.30 \downarrow \\
0.15\end{array}$ & & $\begin{array}{c}300 \downarrow \\
150\end{array}$ & & & 2 & Y & $\mathrm{N}$ & Similar \\
\hline & $E(Y) 2 / S A G A$ & $\mathrm{~N}$ & $\mathrm{~N}$ & & $\begin{array}{c}0.3 \downarrow \\
0.1\end{array}$ & & & $\begin{array}{c}420 \downarrow \\
140\end{array}$ & & 10 & $\begin{array}{c}1.0 \downarrow \\
0.3 \\
\end{array}$ & Y & $\mathrm{Y}$ & $500 \mathrm{mM} \mathrm{KCl}$ \\
\hline & Emerin/Various Interactors & $\mathrm{N}$ & $\mathrm{N}$ & & & $\begin{array}{c}1.0 \downarrow \\
0.1 \\
\end{array}$ & & $\begin{array}{l}1000 \\
\downarrow 100 \\
\end{array}$ & & & & $\mathrm{~N}$ & $\mathrm{~N}$ & Similar \\
\hline & LAP1/LMNB & $\mathrm{N}$ & $\mathrm{N}$ & & & $\begin{array}{c}2.0 \downarrow \\
1.0\end{array}$ & & $\begin{array}{c}300 \downarrow \\
150\end{array}$ & & & $\begin{array}{c}5.0 \downarrow \\
2.5\end{array}$ & $\mathrm{~N}$ & $\mathrm{~N}$ & Similar \\
\hline \multirow{5}{*}{$\begin{array}{l}\frac{ \pm}{\pi} \\
8 \\
5 \\
\frac{1}{3} \\
0\end{array}$} & $\mathrm{BAF}^{\text {Vitro }} /$ Various Interactors & $\mathrm{N}$ & $\mathrm{N}$ & & $0.5^{*}$ & 0.1 & & 150 & & & 2 & $\mathrm{Y}$ & $\mathrm{N}$ & Similar \\
\hline & Emerin $^{\text {Vitro }} /$ Various Interactors & $\mathrm{N}$ & $\mathrm{N}$ & & & 0.2 & & & 20 & 25 & 0.5 & $\mathrm{~N}$ & $\mathrm{~N}$ & $\begin{array}{c}250 \mathrm{mM} \mathrm{NaCl} ; \\
0.1 \% \mathrm{Trx}-100\end{array}$ \\
\hline & LMNC $^{\text {Vitro } / M O K 2 ~}$ & $\mathrm{~N}$ & $\mathrm{~N}$ & & 0.1 & & & $\begin{array}{c}420 \downarrow \\
150\end{array}$ & & 25 & 0.5 & $\mathrm{~N}$ & $\mathrm{~N}$ & Similar \\
\hline & $\begin{array}{c}\text { XLAP2 }^{\text {Vitro }} / \text { LMNA/LMNB1/ } \\
\text { LMNB2 }\end{array}$ & $\mathrm{N}$ & $\mathrm{N}$ & & & 1.0 & & 150 & & & & $\mathrm{~N}$ & $\mathrm{~N}$ & Similar \\
\hline & Nesprin2 ${ }^{\text {Vitro }} /$ LMNA/LMNC & $\mathrm{N}$ & $\mathrm{N}$ & & & 1.0 & & 150 & & & & $\mathrm{~N}$ & $\mathrm{~N}$ & Similar \\
\hline \multirow{2}{*}{ 占 $\frac{\frac{c}{3}}{\bar{z}}$} & LMNA/RBBP4 & $\mathrm{Y}$ & $\mathrm{Y}$ & $\begin{array}{c}1.0 \downarrow \\
0.1\end{array}$ & & 1.0 & & 150 & & & & $\mathrm{~N}$ & $\mathrm{~N}$ & $\begin{array}{l}2 \mathrm{M} \mathrm{NaCl} \\
2 \% \operatorname{Trx}-100\end{array}$ \\
\hline & $\begin{array}{l}\text { LMNA/Progerin/Various } \\
\text { Interactors }\end{array}$ & $\mathrm{Y}$ & $\mathrm{Y}$ & $\begin{array}{c}1.0 \downarrow \\
0.2\end{array}$ & & 1.0 & & 150 & & & & $\mathrm{~N}$ & $\mathrm{~N}$ & $\begin{array}{l}2 \mathrm{M} \mathrm{NaCl} \\
2 \% \operatorname{Trx}-100\end{array}$ \\
\hline
\end{tabular}




\begin{tabular}{|c|c|}
\hline Comments & 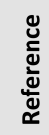 \\
\hline - Antiserum recognizes MAN1, LAP2 $\beta$ and unidentified NE epitopes. & (36) \\
\hline \multirow[t]{2}{*}{ - LMNA antibodies preferentially precipitates LMNA compared to LMNC } & $(12)$ \\
\hline & $(72)$ \\
\hline \multirow[t]{2}{*}{ - LMNA AB also (reduced) affinity for preLMNA } & (38) \\
\hline & $(5)$ \\
\hline \multirow[t]{2}{*}{$\begin{array}{l}\text { - Washes up to } 300 \mathrm{mM} \mathrm{NaCl} \text { resulted in complex dissociation; } \\
\text { - Protein domains were expressed and used for IP as well } \\
\text { - LAP2 } \beta \text { for } 90 \% \text { solubilized, LMNA, LMNB1,2 for app. 50\% } \\
\text { - Certain antibodies not usable as they disrupt Lamin-LAP2 } \beta \text { interactions }\end{array}$} & $(40)$ \\
\hline & $(13)$ \\
\hline - Very small part of solubilized Nesprin2 was precipitated & (45) \\
\hline $\begin{array}{l}-0.1 \% \text { SDS wash fully disrupts p250 and p62 interaction and leaves appr. a } \\
1 / 3^{\text {rd }} \text { of the Nup153 interaction intact }\end{array}$ & $(41)$ \\
\hline $\begin{array}{l}\text { - *Cells were pre-extracted by } 0.1 \% \text { NP-40 and } 1.5 \mathrm{M} \mathrm{KCl} \text {, insoluble pellet } \\
\text { was lysed in described lysis buffer (without NP40 and KCl) }\end{array}$ & (43) \\
\hline \multirow{3}{*}{$\begin{array}{l}\text { - Further increasing } \mathrm{NaCl} \text { up to } 0.5 \mathrm{M} \text { did not drastically improve NPC } \\
\text { solubilization. } \\
\text { - Increasing Trx-100 from } 1 \% \text { to } 2 \% \text { increased NPC solubility from } 50 \text { to } 80 \% \\
\end{array}$} & $(42)$ \\
\hline & (44) \\
\hline & $(62)$ \\
\hline - Novel interactors identified by MS & (33) \\
\hline $\begin{array}{l}\text { - } 10 \% \text { sucrose was included as well in lysis buffer } \\
\text { - Lysing directly in lysis buffer with } 1 \% \text { Trx-100, } 150 \mathrm{mM} \mathrm{NaCl} \text { resulted in } \\
\text { worse solubilization of membrane proteins. }\end{array}$ & (71) \\
\hline $\begin{array}{l}\text { - }{ }^{*} \text { Cells were pre-extracted by } 0.5 \% \text { NP40 } \\
\text { - Novel interactors identified by MS }\end{array}$ & (44) \\
\hline $\begin{array}{l}\text { - Novel interactors identified by MS } \\
\text { - Only residues 1-122 of emerin were translated }\end{array}$ & (33) \\
\hline $\begin{array}{l}\text { - Protein complex re-assembled due to dialysis of lysis buffer. Listed within } \\
\text { this category because LMNC was translated in vitro }\end{array}$ & $(72)$ \\
\hline \multirow[t]{3}{*}{ - Protein domains were in vitro translated and used for IP as well } & $(40)$ \\
\hline & $(45)$ \\
\hline & (6) \\
\hline - Novel interactors identified by MS & (46) \\
\hline
\end{tabular}


Table 3: Techniques to identify chromatin interactions at the nuclear lamina

\begin{tabular}{|c|c|c|c|c|c|c|c|c|}
\hline \multirow{2}{*}{ 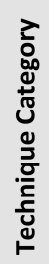 } & \multirow[b]{2}{*}{ Technique } & \multicolumn{2}{|c|}{ System } & \multicolumn{2}{|c|}{$\begin{array}{l}\text { Studied } \\
\text { proteins }\end{array}$} & \multicolumn{2}{|c|}{ Captures } & \multirow[b]{2}{*}{ Advantages } \\
\hline & & $\underset{\mathfrak{s}}{\stackrel{\rho}{\mathfrak{s}}}$ & $\begin{array}{l}\stackrel{0}{0} \\
\sum_{0}^{0} \\
\frac{0}{\delta} \\
0 \\
\frac{0}{0} \\
0\end{array}$ & 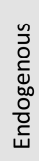 & 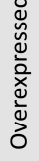 & $\begin{array}{l}\frac{\mathrm{o}}{\mathrm{n}} \\
\frac{0}{0} \\
\text { जั }\end{array}$ & 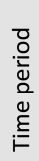 & \\
\hline \multirow{3}{*}{ 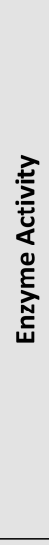 } & DamID & + & + & - & + & - & + & $\begin{array}{l}\text { - No need for solubilization of protein/DNA complexes } \\
\text { - No formaldehyde fixation required }\end{array}$ \\
\hline & $\begin{array}{l}\text { In vivo ChEC } \\
\text { (Chromatin Endogenous } \\
\text { Cleavage) }\end{array}$ & + & + & - & + & \pm & \pm & $\begin{array}{l}\text { - No need for solubilization of protein/DNA complexes } \\
\text { - No formaldehyde fixation required } \\
\text { - Southern blot analysis provides extra information } \\
\text { about hypersensitive sights }\end{array}$ \\
\hline & $\begin{array}{c}\text { ChlC } \\
\text { (Chromatin } \\
\text { Immunocleavage) }\end{array}$ & + & + & + & + & + & - & $\begin{array}{l}\text { - No need for solubilization of protein/DNA complexes } \\
\text { - Southern blot analysis provides extra information } \\
\text { about hypersensitive sights } \\
\text { - Possible to study rapid changes of interaction } \\
\text { - Endogenous enzymatic activity impossible } \\
\text { - Antibody allows to study posttranslational } \\
\text { modifications }\end{array}$ \\
\hline \multirow{2}{*}{ Dूd } & $\begin{array}{c}\text { Chromatin } \\
\text { Immunoprecipitation }\end{array}$ & + & + & + & + & + & - & $\begin{array}{l}\text { - Antibody allows to study posttranslational } \\
\text { modifications } \\
\text { - Possible to study rapid changes of interaction }\end{array}$ \\
\hline & OneSTrEP Pulldown & + & + & - & + & + & - & $\begin{array}{l}\text { - High affinity precipitation without need for aAntibody } \\
\text { - Near complete solubilization of protein/DNA } \\
\text { complexes possible }\end{array}$ \\
\hline
\end{tabular}

Legend: No (-), Moderate time period ( \pm ), Yes (+) 


\begin{tabular}{|c|c|c|}
\hline Disadvantages & Extra Information & References \\
\hline $\begin{array}{l}\text { - Relative low resolution } \\
\text { - Target protein needs to be expressed at very low } \\
\text { levels } \\
\text { - Not possible to study posttranslational } \\
\text { modifications }\end{array}$ & $\begin{array}{l}\text { - Tag is active under } \\
\text { physiological } \\
\text { conditions } \\
\text { - Resolution of } 1000 \\
\text { bp } \\
\text { - Dam-tag } \sim 32 \mathrm{kDa} \\
\end{array}$ & $(59,61)$ \\
\hline $\begin{array}{l}\text { - Dependent on the presence of DNA restriction sites } \\
\text { - Target protein cannot be expressed in high levels } \\
\text { - Not possible to study posttranslational } \\
\text { modifications }\end{array}$ & $\begin{array}{l}\text { - Tag is inactive under } \\
\text { physiological } \\
\text { conditions } \\
\text { - Resolution of 100bp } \\
\text { - MN-tag 18kDa } \\
\end{array}$ & $(64-65)$ \\
\hline $\begin{array}{l}\text { - Dependent on the presence of DNA restriction sites } \\
\text { - Interacting DNA that cannot be reached by tag } \\
\text { enzymatic activity remains undetected } \\
\text { - Antibodies may need separate optimization } \\
\text { - Formaldehyde fixation required }\end{array}$ & $\begin{array}{l}\text { - Tag is inactive under } \\
\text { physiological } \\
\text { conditions } \\
\text { - Resolution of } 100 \mathrm{bp}\end{array}$ & $(64-65)$ \\
\hline $\begin{array}{l}\text { - Dependent on antibody quality } \\
\text { - Formaldehyde fixation required } \\
\text { - Solubilization of protein/DNA complexes required }\end{array}$ & - Resolution of $\sim 500 \mathrm{bp}$ & $\begin{array}{l}(54,57,76- \\
79)\end{array}$ \\
\hline $\begin{array}{l}\text { - Formaldehyde fixation required } \\
\text { - Not possible to study posttranslational } \\
\text { modifications }\end{array}$ & $\begin{array}{l}\text { - Resolution of } \sim 500 \mathrm{bp} \\
\text { - OST-tag } \sim 3 \mathrm{kDa}\end{array}$ & (58) \\
\hline
\end{tabular}




\section{References}

1. Foisner R. Dynamic Connections of Nuclear Envelope Proteins to Chromatin and the Nuclear Matrix. In: Collas P, ed. Nuclear Envelope Dynamics in Embryos and Somatic Cells, 2003.

2. Mattout-Drubezki A, Gruenbaum Y. Dynamic interactions of nuclear lamina proteins with chromatin and transcriptional machinery. Cell Mol Life Sci 2003; 60:2053-63.

3. Broers JL, Hutchison CJ, Ramaekers FC. Laminopathies. J Pathol 2004; 204:478-88.

4. Broers JL, Ramaekers FC, Bonne G, Yaou RB, Hutchison CJ. Nuclear lamins: laminopathies and their role in premature ageing. Physiol Rev 2006; 86:967-1008.

5. Lloyd DJ, Trembath RC, Shackleton S. A novel interaction between lamin A and SREBP1: implications for parial lipodystrophy and other laminopathies. Human Molecular Genetics 2002; 11:769-77.

6. Pegoraro G, Kubben N, Wickert U, Gohler H, Hoffmann K, Misteli T. Ageing-related chromatin defects through loss of the NURD complex. Nat Cell Biol 2009; 11:1261-7.

7. Taimen P, Pfleghaar K, Shimi T, Moller D, Ben-Harush K, Erdos MR, et al. A progeria mutation reveals functions for lamin $A$ in nuclear assembly, architecture, and chromosome organization. Proc Natl Acad Sci U S A 2009; 106:20788-93.

8. $\mathrm{Wu} \mathrm{CC}$, Yates JR, 3rd. The application of mass spectrometry to membrane proteomics. Nat Biotechnol 2003; 21:262-7.

9. Yates JR, Ruse $\mathrm{Cl}$, Nakorchevsky A. Proteomics by mass spectrometry: approaches, advances, and applications. Annu Rev Biomed Eng 2009; 11:49-79.

10. Werner T. Next generation sequencing in functional genomics. Brief Bioinform 2010.

11. Sutherland HG, Mumford GK, Newton K, Ford LV, Farrall R, Dellaire G, et al. Largescale identification of mammalian proteins localized to nuclear sub-compartments. Hum Mol Genet 2001; 10:1995-2011.

12. Van Berlo JH, Voncken JW, Kubben N, Broers JL, Duisters R, van Leeuwen RE, et al. Atype lamins are essential for TGF-beta1 induced PP2A to dephosphorylate transcription factors. Hum Mol Genet 2005; 14:2839-49.

13. Ivorra C, Kubicek M, Gonzalez JM, Sanz-Gonzalez SM, Alvarez-Barrientos A, O'Connor $\mathrm{JE}$, et al. A mechanism of AP-1 suppression through interaction of c-Fos with lamin A/C. Genes Dev 2006; 20:307-20.

14. Ross-Macdonald P, Coelho PS, Roemer T, Agarwal S, Kumar A, Jansen R, et al. Largescale analysis of the yeast genome by transposon tagging and gene disruption. Nature 1999; 402:413-8.

15. Kumar A, Agarwal S, Heyman JA, Matson S, Heidtman M, Piccirillo S, et al. Subcellular localization of the yeast proteome. Genes Dev 2002; 16:707-19.

16. Simpson JC, Wellenreuther R, Poustka A, Pepperkok R, Wiemann S. Systematic subcellular localization of novel proteins identified by large-scale cDNA sequencing. EMBO Rep 2000; 1:287-92.

17. Simpson JC, Pepperkok R. Localizing the proteome. Genome Biol 2003; 4:240.

18. Kahsay RY, Gao G, Liao L. An improved hidden Markov model for transmembrane protein detection and topology prediction and its applications to complete genomes. Bioinformatics 2005; 21:1853-8.

19. Shen HB, Chou KC. Nuc-PLoc: a new web-server for predicting protein subnuclear localization by fusing PseAA composition and PsePSSM. Protein Eng Des Sel 2007; 20:561-7. 
20. Rolls MM, Stein PA, Taylor SS, Ha E, McKeon F, Rapoport TA. A visual screen of a GFPfusion library identifies a new type of nuclear envelope membrane protein. J Cell Biol 1999; 146:29-44.

21. Hofemeister $\mathrm{H}, \mathrm{O}$ 'Hare P. Analysis of the localization and topology of nurim, a polytopic protein tightly associated with the inner nuclear membrane. J Biol Chem 2005; 280:2512-21.

22. Huh WK, Falvo JV, Gerke LC, Carroll AS, Howson RW, Weissman JS, et al. Global analysis of protein localization in budding yeast. Nature 2003; 425:686-91.

23. Thirkettle HJ, Mills IG, Whitaker HC, Neal DE. Nuclear LYRIC/AEG-1 interacts with PLZF and relieves PLZF-mediated repression. Oncogene 2009; 28:3663-70.

24. Wu CC, MacCoss MJ, Howell KE, Yates JR, 3rd. A method for the comprehensive proteomic analysis of membrane proteins. Nat Biotechnol 2003; 21:532-8.

25. Foster $\mathrm{L}$, de Hoog $\mathrm{CL}$, Zhang $\mathrm{Y}$, Zhang $\mathrm{Y}$, Xie X, Mootha VK, et al. A mammalian organelle map by protein correlation profiling. Cell 2006; 125:187-99.

26. Clawson GA, Moody DE, James J, Smuckler EA. Nuclear envelope alterations accompanying thioacetamide-related enlargement of the nucleus. Cancer Res 1981; 41:519-26.

27. Cronshaw JM, Krutchinsky AN, Zhang W, Chait BT, Matunis MJ. Proteomic analysis of the mammalian nuclear pore complex. J Cell Biol 2002; 158:915-27.

28. Dreger M, Bengtsson L, Schoneberg T, Otto H, Hucho F. Nuclear envelope proteomics: novel integral membrane proteins of the inner nuclear membrane. Proc Natl Acad Sci U S A 2001; 98:11943-8.

29. Korfali N, Fairley EA, Swanson SK, Florens L, Schirmer EC. Use of sequential chemical extractions to purify nuclear membrane proteins for proteomics identification. Methods Mol Biol 2009; 528:201-25.

30. Sutherland HG, Lam YW, Briers S, Lamond AI, Bickmore WA. 3D3/lyric: a novel transmembrane protein of the endoplasmic reticulum and nuclear envelope, which is also present in the nucleolus. Exp Cell Res 2004; 294:94-105.

31. Siniossoglou S, Wimmer C, Rieger M, Doye V, Tekotte $H$, Weise $C$, et al. A novel complex of nucleoporins, which includes Sec13p and a Sec13p homolog, is essential for normal nuclear pores. Cell 1996; 84:265-75.

32. Goodchild RE, Dauer WT. Mislocalization to the nuclear envelope: an effect of the dystonia-causing torsinA mutation. Proc Natl Acad Sci U S A 2004; 101:847-52.

33. Holaska JM, Wilson KL. An emerin "proteome": purification of distinct emerincontaining complexes from HeLa cells suggests molecular basis for diverse roles including gene regulation, mRNA splicing, signaling, mechanosensing, and nuclear architecture. Biochemistry 2007; 46:8897-908.

34. Nikolakaki E, Simos G, Georgatos SD, Giannakouros T. A nuclear envelope-associated kinase phosphorylates arginine-serine motifs and modulates interactions between the lamin B receptor and other nuclear proteins. J Biol Chem 1996; 271:8365-72.

35. Muralikrishna B, Thanumalayan S, Jagatheesan G, Rangaraj N, Karande AA, Parnaik VK. Immunolocalization of detergent-susceptible nucleoplasmic lamin A/C foci by a novel monoclonal antibody. J Cell Biochem 2004; 91:730-9.

36. Lin F, Morrison JM, Wu W, Worman HJ. MAN1, an integral protein of the inner nuclear membrane, binds Smad2 and Smad3 and antagonizes transforming growth factor-beta signaling. Hum Mol Genet 2005; 14:437-45.

37. Zhong N, Radu G, Ju W, Brown WT. Novel progerin-interactive partner proteins hnRNP E1, EGF, Mel 18, and UBC9 interact with lamin A/C. Biochem Biophys Res Commun 2005; 338:855-61. 
38. Capanni C, Mattioli E, Columbaro M, Lucarelli E, Parnaik VK, Novelli G, et al. Altered pre-lamin A processing is a common mechanism leading to lipodystrophy. Hum Mol Genet 2005; 14:1489-502.

39. Farnum M, Zukoski C. Effect of glycerol on the interactions and solubility of bovine pancreatic trypsin inhibitor. Biophys J 1999; 76:2716-26.

40. Lang C, Krohne G. Lamina-associated polypeptide 2beta (LAP2beta) is contained in a protein complex together with A- and B-type lamins. Eur J Cell Biol 2003; 82:143-53.

41. Grandi P, Dang T, Pane N, Shevchenko A, Mann M, Forbes D, et al. Nup93, a vertebrate homologue of yeast Nic96p, forms a complex with a novel 205-kDa protein and is required for correct nuclear pore assembly. Mol Biol Cell 1997; 8:201738.

42. Zhang SX, Garcia-Gras E, Wycuff DR, Marriot SJ, Kadeer N, Yu W, et al. Identification of direct serum-response factor gene targets during Me2SO-induced P19 cardiac cell differentiation. J Biol Chem 2005; 280:19115-26.

43. Doufexis M, Storr HL, King PJ, Clark AJ. Interaction of the melanocortin 2 receptor with nucleoporin 50: evidence for a novel pathway between a G-protein-coupled receptor and the nucleus. FASEB J 2007; 21:4095-100.

44. Montes de Oca R, Shoemaker CJ, Gucek M, Cole RNW, K.L. Barrier-to-Autointegration Factor Proteome Reveals Chromatin-Regulatory Partners. PLoS One 2009; 4:e7050.

45. Libotte T, Zaim H, Abraham S, Padmakumar VC, Schneider M, Lu W, et al. Lamin A/Cdependent localization of Nesprin-2, a giant scaffolder at the nuclear envelope. Mol Biol Cell 2005; 16:3411-24.

46. Kubben N, Voncken J, Demmers JA, Calis C, van Almen G, Misteli T, et al. In preparation: Novel distinct protein interactions of lamin A and progerin., 2010.

47. Junttila MR, Saarinen S, Schmidt T, Kast J, Westermarck J. Single-step Strep-tag purification for the isolation and identification of protein complexes from mammalian cells. Proteomics 2005; 5:1199-203.

48. Witte CP, Noel LD, Gielbert J, Parker JE, Romeis T. Rapid one-step protein purification from plant material using the eight-amino acid Strepll epitope. Plant Mol Biol 2004; 55:135-47.

49. Sakaki M, Koike H, Takahashi N, Sasagawa N, Tomioka S, Arahata K, et al. Interaction between emerin and nuclear lamins. J Biochem 2001; 129:321-7.

50. Goldberg M, Lu H, Stuurman N, Ashery-Padan R, Weiss AM, Yu J, et al. Interactions among Drosophila nuclear envelope proteins lamin, otefin, and YA. Mol Cell Biol 1998; 18:4315-23.

51. Maraldi NM, Lattanzi G, Capanni C, Columbaro M, Mattioli E, Sabatelli $P$, et al. Laminopathies: a chromatin affair. Adv Enzyme Regul 2006; 46:33-49.

52. Ruault $M$, Dubarry $M$, Taddei $A$. Re-positioning genes to the nuclear envelope in mammalian cells: impact on transcription. Trends Genet 2008; 24:574-81.

53. Schneider R, Grosschedl R. Dynamics and interplay of nuclear architecture, genome organization, and gene expression. Genes Dev 2007; 21:3027-43.

54. Casolari JM, Brown CR, Komili S, West J, Hieronymus H, Silver PA. Genome-wide localization of the nuclear transport machinery couples transcriptional status and nuclear organization. Cell 2004; 117:427-39.

55. Partridge JF, Borgstrom B, Allshire RC. Distinct protein interaction domains and protein spreading in a complex centromere. Genes Dev 2000; 14:783-91.

56. Grund SE, Fischer T, Cabal GG, Antunez O, Perez-Ortin JE, Hurt E. The inner nuclear membrane protein Src1 associates with subtelomeric genes and alters their regulated gene expression. J Cell Biol 2008; 182:897-910. 
57. Brown CR, Kennedy CJ, Delmar VA, Forbes DJ, Silver PA. Global histone acetylation induces functional genomic reorganization at mammalian nuclear pore complexes. Genes Dev 2008; 22:627-39.

58. Kubben N, Adriaens M, Voncken J, Pinto $\mathrm{Y}$, Misteli T. In preparation: Specific interactions of genome regions with lamin $A$ and progerin., 2010.

59. Pickersgill H, Kalverda B, de Wit E, Talhout W, Fornerod M, van Steensel B. Characterization of the Drosophila melanogaster genome at the nuclear lamina. Nat Genet 2006; 38:1005-14.

60. Guelen L, Pagie L, Brasset E, Meuleman W, Faza MB, Talhout W, et al. Domain organization of human chromosomes revealed by mapping of nuclear lamina interactions. Nature 2008; 453:948-51.

61. Vogel MJ, Peric-Hupkes D, van Steensel B. Detection of in vivo protein-DNA interactions using DamID in mammalian cells. Nat Protoc 2007; 2:1467-78.

62. Kurshakova MM, Krasnov AN, Kopytova DV, Shidlovskii YV, Nikolenko JV, Nabirochkina EN, et al. SAGA and a novel Drosophila export complex anchor efficient transcription and mRNA export to NPC. EMBO J 2007; 26:4956-65.

63. Scaffidi $P$, Misteli T. Reversal of the cellular phenotype in the premature aging disease Hutchinson-Gilford progeria syndrome. Nat Med 2005; 11:440-5.

64. Schmid M, Arib G, Laemmli C, Nishikawa J, Durussel T, Laemmli UK. Nup-PI: the nucleopore-promoter interaction of genes in yeast. Mol Cell 2006; 21:379-91.

65. Schmid M, Durussel T, Laemmli UK. ChIC and ChEC; genomic mapping of chromatin proteins. Mol Cell 2004; 16:147-57.

66. Schirmer EC, Gerace L. The nuclear membrane proteome: extending the envelope. Trends Biochem Sci 2005.

67. Fink JL, Karunaratne S, Mittal A, Gardiner DM, Hamilton N, Mahony D, et al. Towards defining the nuclear proteome. Genome Biol 2008; 9:R15.

68. Miller BR, Forbes DJ. Purification of the vertebrate nuclear pore complex by biochemical criteria. Traffic 2000; 1:941-51.

69. Schirmer EC, Florens L, Guan T, Yates JR, 3rd, Gerace L. Nuclear membrane proteins with potential disease links found by subtractive proteomics. Science 2003; 301:13802.

70. Batrakou DG, Kerr AR, Schirmer EC. Comparative proteomic analyses of the nuclear envelope and pore complex suggests a wide range of heretofore unexpected functions. J Proteomics 2009; 72:56-70.

71. Maison C, Pyrpasopoulou A, Theodoropoulos PA, Georgatos SD. The inner nuclear membrane protein LAP1 forms a native complex with B-type lamins and partitions with spindle-associated mitotic vesicles. The EMBO Journal 1997; 16:4839-50.

72. Dreuillet $C$, Tillit J, Kress $M$, Ernoult-Lange $M$. In vivo and in vitro interaction between human transcription factor MOK2 and nuclear lamin A/C. Nucleic Acids Res 2002; 30:4634-42.

73. Wagner N, Weber D, Seitz S, Krohne G. The lamin B receptor of Drosophila melanogaster. J Cell Sci 2004; 117:2015-28.

74. Rasala BA, Orjalo AV, Shen Z, Briggs S, Forbes DJ. ELYS is a dual nucleoporin/kinetochore protein required for nuclear pore assembly and proper cell division. Proc Natl Acad Sci U S A 2006; 103:17801-6.

75. Apel ED, Lewis RM, Grady RM, Sanes JR. Syne-1, a dystrophin- and Klarsicht-related protein associated with synaptic nuclei at the neuromuscular junction. J Biol Chem 2000; 275:31986-95. 
76. Menon BB, Sarma NJ, Pasula S, Deminoff SJ, Willis KA, Barbara KE, et al. Reverse recruitment: the Nup84 nuclear pore subcomplex mediates Rap1/Gcr1/Gcr2 transcriptional activation. Proc Natl Acad Sci U S A 2005; 102:5749-54.

77. Casolari JM, Brown CR, Drubin DA, Rando OJ, Silver PA. Developmentally induced changes in transcriptional program alter spatial organization across chromosomes. Genes Dev 2005; 19:1188-98.

78. Cabal GG, Genovesio A, Rodriguez-Navarro S, Zimmer C, Gadal O, Lesne A, et al. SAGA interacting factors confine sub-diffusion of transcribed genes to the nuclear envelope. Nature 2006; 441:770-3.

79. Schirmer EC. The epigenetics of nuclear envelope organization and disease. Mutat Res 2008; 647:112-21. 


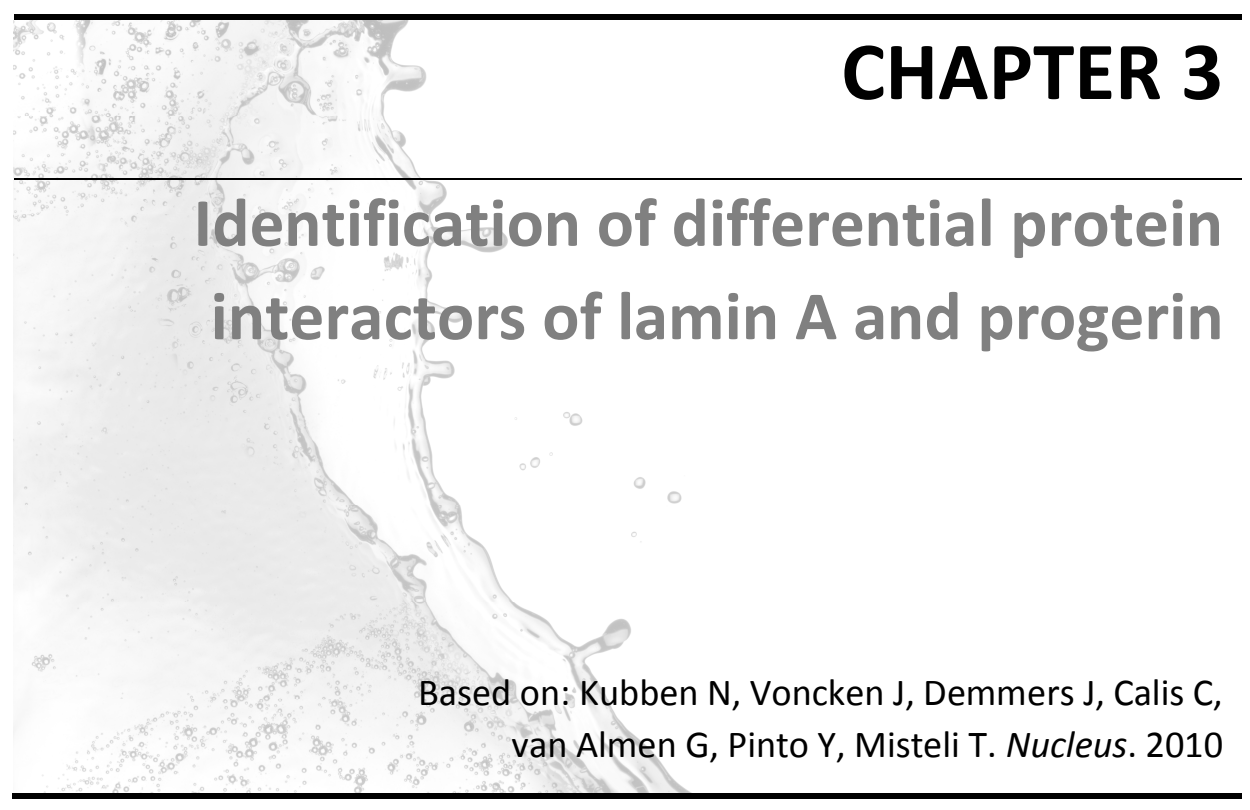




\begin{abstract}
The nuclear lamina is an interconnected meshwork of intermediate filament proteins underlying the nuclear envelope. The lamina is an important regulator of nuclear structural integrity as well as various nuclear processes, including transcription, DNA replication and chromatin remodeling. The major components of the lamina are A-type and B-type lamins. Mutations in lamins impair lamina functions and cause a set of highly tissue-specific diseases collectively referred to as laminopathies. The phenotypic diversity amongst laminopathies is hypothesized to be caused by mutations affecting specific protein interactions, possibly in a tissue-specific manner. Current technologies to identify interaction partners of lamin A and its mutants are hampered by the insoluble nature of lamin components. To overcome the limitations of current technologies, we developed and applied a novel, unbiased approach to identify lamin A-interacting proteins. This approach involves expression of the high-affinity OneSTrEP-tag, precipitation of laminprotein complexes after reversible protein cross-linking and subsequent protein identification by mass spectrometry. We used this approach to identify in mouse embryonic fibroblasts and cardiac myocyte NkITAg cell lines proteins that interact with lamin A and its mutant isoform progerin, which causes the premature aging disorder Hutchinson-Gilford Progeria Syndrome (HGPS). We identified a total of 313 lamina-interacting proteins, including several novel lamin A interactors, and we characterize a set of 35 proteins which preferentially interact with lamin A or progerin.
\end{abstract}




\section{Introduction}

The nuclear lamina lines the nuclear envelope (NE), which is composed of an outer and inner nuclear membrane (ONM, INM,) interrupted by nuclear pore complexes (NPC). ${ }^{1}$ The lamina is a proteinaceous structure consisting of the intermediate filament A-type (somatic: lamin $A, A \Delta 10, C$; germ cell specific: C2) and B-type lamins (somatic: lamin B1, B2; germ cell specific: $B 3)$, integral membrane proteins anchoring the lamina to the $N E$ including LAP2, Emerin, Lamin B receptor, MAN1, and proteins involved in a variety of nuclear processes including DNA replication, chromatin remodeling, cell cycle progression and signal transduction. ${ }^{2}$ Mutations in lamins or other lamina proteins can impair specific functions or interfere with the structural integrity of the nucleus. ${ }^{3}$ Mutations in lamin components cause various human diseases referred to as laminopathies and include several types of muscular dystrophies, lipodystrophies, cardiomyopathies, neurological disorders and premature aging syndromes. ${ }^{2}$

One model for the tissue-specificity and phenotypic diversity among laminopathies is the notion that mutations which cause laminopathies lead to differential interactions of mutant lamins with their associated proteins. ${ }^{2}$ In support, several mutations in the carboxy-terminus of lamin A that lead to Emery-Dreifuss muscular dystrophy (EDMD) or dilated cardiomyopathy $1 \mathrm{~A}$ (CMD1A) impair a direct binding of lamin $A$ to emerin. ${ }^{4}$ In addition, progerin, the lamin $A$ isoform that causes the premature aging disease Hutchinson-Gilford Progeria Syndrome (HGPS), is unable to interact with several members of the NURD chromatin remodeling complex, thereby contributing to HGPS characteristic chromatin defects. ${ }^{5}$ Although these observations suggest a role for differential protein interactions in the tissuespecificity of laminopathies, they are circumstantial. Systematic mapping of the complete spectrum of wild-type and mutant lamin A interacting proteins is required to test this disease model. Such approaches will also clarify the function of lamin A in biological processes and may contribute to the identification of targetable pathways relevant to specific laminopathies.

Experimental approaches to identify lamin interacting proteins are severely hampered by the unique biochemical properties of the nuclear lamina, which is classically defined as a non-ionic detergent, salt and nuclease 
insoluble peripheral nuclear structure. ${ }^{1}$ Solubilization of the lamina by ultrasonication and high concentrations of ionic- or chaotropic detergents inevitably results in disruption of intact lamin-protein complexes, impeding analysis by precipitation methods. Various alternative strategies have been applied to circumvent these technical problems, including immunoprecipitation (IP) with partial solubilization under mild conditions, IP using harsh solubilization conditions followed by in vitro protein complex reassembly under mild conditions, mass spectrometry (MS) analysis of differential extractions from isolated whole nuclear membrane fractions, and yeast two-hybrid, GST-pull-downs and other techniques which do not need protein complex solubilization. ${ }^{6-12}$ Each technology has its specific drawbacks including low recovery, high false positive rates and nonphysiological conditions.

Here we have developed and applied a novel method to identify wild-type and mutant lamin A interacting proteins. This approach is unbiased and overcomes many of the limitations of current methods to identify lamin Ainteracting proteins. Our approach consists of expression of lamin A tagged with a OneSTrEP biotin analogue tag followed by isolation of intact laminprotein complexes after protein cross-linking for 5 minutes using 1\% formaldehyde. Direct and indirect interaction partners are identified by sensitive MS after a highly specific streptactin matrix precipitation. Using this approach we identify 313 lamina-interacting proteins including numerous novel candidates, and proteins that appear to associate preferentially with either wildtype lamin A (8 candidates, including Nup153, Nup98 and Translocated promoter region) or progerin (27 candidates, including Paired related homeobox 1, Proliferating cell nuclear antigen and Pinin).

\section{Materials \& Methods}

\section{Cell lines and culture}

A lentiviral expression plasmid for $\mathrm{N}$-terminal OneSTrEP tagged progerin (OST-P) was created by PCR amplification of lamin A, omitting amino acids 609-658 using the previously described PCDH MCSNard OST-A plasmid as template. ${ }^{5}$ Forward and reverse primers, containing a BamHI and EcoRI 
restriction site, were used for subsequent ligation of the PCR product in BamHI/EcoRI digested pCDH MCSNard OST-A plasmid. For the cloning of pCDH MCSNard OST-Lamin C (OST-C), lamin C was PCR amplified from the previously described pBabe purox HA-Lamin $C$ plasmid, adding an in frame 5' Sall and a 3' EcoRI site, which were used for ligation into equally digested pCDH MCSNard OST-A plasmid. ${ }^{13}$ Lentivirus was produced in $293 \mathrm{FT}$ cells (Invitrogen, Carlsbad, USA) co-transfected with pCDHblast MCSNard OST-A, OST-P or OST-C in combination with PSPAX and PMD2.G vectors (Trono Lab). The mouse cardiac myocyte cell line NkITAg, human osteosarcoma cell (U2OS), as well as mouse embryonic fibroblasts (MEFs) of wildtype $\left(\mathrm{LMNA}^{+/+}\right)$and LMNA knock-out $\left(\mathrm{LMNA}^{-/}\right)$embryos were infected with various concentrations of lentivirus and 48 hours post infection selected with blasticidin. ${ }^{14-15}$ Infected cells with OST-tagged lamin protein amounts comparable to endogenous lamin protein levels were further cultured and grown in Dulbecco's modified Eagle's/F-12 media (for NkITAg cells) or Dulbecco's modified eagle medium (for U2OS and MEFs) both supplemented with $2 \mathrm{mM}$ L-glutamine, $10 \mathrm{mM}$ non-essential amino acids, $110 \mathrm{mg} / \mathrm{l}$ pyruvate, $10 \%$ fetal bovine serum and antibiotics in a humidified atmosphere at $37^{\circ} \mathrm{C}$ and $5 \% \mathrm{CO}_{2}$. NkITAg cells were plated on $12.5 \mathrm{\mu g} / \mathrm{ml}$ fibronectin and $0.1 \%$ gelatin coated plates.

\section{Immunofluorescence}

For immunofluorescence microscopy LMNA ${ }^{-/}$MEFs infected with control, OST-A or OST-P lentivirus were grown on $0.1 \%$ gelatin/PBS solution coated multi-wells glass slides (MP Biomedicals). After fixation (4\% paraformaldehyde/PBS; $15 \mathrm{~min})$, cells were permeabilized (0.5\% Triton X100/PBS; $5 \mathrm{~min}$ ), blocked (1\% BSA, 1\% sucrose in milliQ; 1 hour) and incubated for one hour with appropriate primary antibodies ( $\alpha$-LMNA/C, Sc7292, Mouse, Santa Cruz, detects OST-tagged and endogenous human, but not mouse, lamin A, Lamin C and Progerin; $\alpha$-emerin, Cl.4G5, mouse, Leica; $\alpha-H P 1 \gamma$, mab3450, Chemicon, Temecula, USA ; $\alpha$-LAP2, kind gift from K. Wilson, raised against residues 1-187 of human LAP2, and suitable for detection of all LAP2 isoforms). Primary antibodies were detected with an Alexa Fluor 488 chromophore labeled secondary antibody (Invitrogen). Cells were mounted in Vectashield containing $10 \mathrm{~g} / \mathrm{ml}$ DAPI and observed on a Nikon E800 microscope. Quantitative microscopy measurements were 
performed as previously described. ${ }^{16-17}$ Exposure times and acquisition settings were established at the beginning of the experiment and kept constant for all samples. The average nuclear intensity of total LAP2 (all isoforms included) and HP1Y minus average background signal was measured for at least 200 cells per sample in duplicate using Metamorph software (Molecular Devices). The criteria to define cells with reduced levels of chromatin proteins were previously described. ${ }^{16-17}$

\section{OneSTrEP-Iamin A farnesylation and proliferative effects}

To determine whether OST-A was correctly farnesylated U2OS cells expressing OST-A and $C$ at endogenous levels were treated with indicated concentrations of Atorvastatin (10 mM stock dissolved in DMSO was further diluted in cell culture medium) for 48 hours. Samples were harvested in Laemmle Sample Buffer and further analyzed by western blotting. Proliferative effects of OST-tagged lamin A/C were determined with the MTS assay, as described elsewhere. ${ }^{13} \mathrm{LMNA}^{-/-} \mathrm{MEF}$ infected with empty control or OST-A and C were seeded at 1000 cells per well in 96 wells plates. ${ }^{15} 62$ hours post attachment $20 \mathrm{ml} 2 \mathrm{mg} / \mathrm{ml} \mathrm{3-(4,5-dimethylthiazol-2-yl)-5-(3-}$ carboxymethoxyphenyl)-2-(4-sulfophenyl)-2H-tetrazolium

(Promega CellTiter 96w AQueous MTS) and $1 \mathrm{ml} 0.92 \mathrm{mg} / \mathrm{ml}$ phenazine methosulfate (Merck) was added for 3 hours. The tetrazolium is reduced by the living cells into a colored formazan product, which absorbance was measured at 490 $\mathrm{nm}$ and is directional proportional to the cell number. Statistical analysis was performed by Student's t-test.

\section{OneSTrEP pull-down assay and mass spectrometry}

U2OS, NkITAg and MEF cell lines were grown until confluency in $150 \mathrm{~mm}$ dishes. Plates were left for several minutes at ambient temperature before protein complexes were mildly cross-linked by direct addition of formaldehyde to the culturing medium to a final concentration of $1 \%$ (10 minutes, ambient temperature). The cross-linking reaction was stopped by addition of glycine ( $125 \mu \mathrm{M}$ final conc.) and gentle shaking for 5 minutes at ambient temperature. Fixed cells were scraped in PBS, pelleted, dissolved in ice-cold SDS lysis buffer (1\% SDS, $10 \mathrm{mM}$ EDTA, $50 \mathrm{mM}$ Tris pH 8.1), snap frozen in dry ice once, thawed, left for 30 minutes on ice and finally sonicated for 2 times 30 seconds at $10 \mu \mathrm{m}$ amplitude using a tip sonicator. 
Endogenous biotinylated proteins were blocked by addition of avidin (10 $\mu \mathrm{g} / \mathrm{ml})$. As the affinity of avidin for biotin is much higher then for the OneSTrEP epitope, OST-tagged lamin pull-downs will not be impaired by the addition of avidin. ${ }^{18} \mathrm{~A}$ small sample aliquot was taken, de-cross-linked for 6 hours at $65^{\circ} \mathrm{C}$ in the presence of $200 \mathrm{mM} \mathrm{NaCl}$ and $30 \mu \mathrm{g} / \mathrm{ml}$ RNAase A, after which the genomic DNA concentration was determined with a Qiagen PCR clean-up kit (Qiagen). Next, protein samples were diluted in SDS lysis buffer

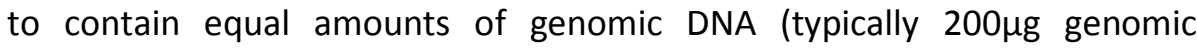
DNA/pull-down for MS purposes) and subsequently diluted in ChIP dilution buffer (0.1\% SDS, 1.1\% Triton X-100, 1.2 mM EDTA, 17 mM Tris pH 8.1, 170 $\mathrm{mM} \mathrm{NaCl}$ ) to reach a final concentration of $0.2 \%$ SDS, $0.1 \%$ Triton X-100, $2 \mathrm{mM}$ EDTA, $20 \mathrm{mM}$ Tris. $\mathrm{HCl}$ pH 8.1, $150 \mathrm{mM} \mathrm{NaCl}$. Both SDS lysis buffer and ChIP dilution buffer were supplemented with $2 \mathrm{mM}$ sodiumortohovanadate and protease inhibitors (Boehringer MannHeim). Undissolved fractions were discarded after a 10 minute centrifugation step $(16,000 \mathrm{~g})$ at 4 degrees and a small sample of the supernatant (i.e. input fraction) was stored for further analysis. ChIP dilution buffer pre-washed Strep-Tactin Matrix (IBATagnology) was added and OST-lamin/protein complexes were bound by overnight endover-end rotation. Strep-Tactin matrix was pelleted by centrifugation and the supernatant (i.e. depleted fraction) was stored, while the pellet was washed four times with OST stringent wash buffer ( $2 \mathrm{M} \mathrm{NaCl}, 2 \% \operatorname{Trx}-100$, $500 \mathrm{mM} \mathrm{LiCl}, 0.1 \%$ SDS, 1\% Sodium Deoxycholate, $20 \mathrm{mM}$ Tris $\mathrm{pH} 8.1,2 \mathrm{mM}$ EDTA) and twice with ChIP dilution buffer. $\mathrm{LiCl}$ was included to improve the effective removal of non-specific interactors by the presence of different types of salts. Pelleted protein-chromatin complexes (pull-down fraction), input and depleted fractions were de-cross-linked by boiling for 20 minutes at $95^{\circ} \mathrm{C}$ in Laemmli Sample Buffer.

For pull-down verification purposes samples were analyzed by western blot (see below). For mass spectrometry (MS) pull-down fractions were separated on NuPage Novex Bis-Tris 4-20\% gradient gels (Invitrogen) and stained with Coomassie Brilliant Blue (CBB). For CBB staining gels were first fixed for minimally 2 hours in solution 1 (50\% v/v ethanol, $2 \% \mathrm{v} / \mathrm{v}$ phosphoric acid), washed $3 \times 30$ minutes in distilled water, incubated for 1 hour in solution 2 ( $34 \% \mathrm{v} / \mathrm{v}$ methanol, $17 \% \mathrm{w} / \mathrm{v}$ ammonium sulphate, $2 \% \mathrm{v} / \mathrm{v}$ phosphoric acid) and finally stained with CBB-G $(0.66 \% \mathrm{w} / \mathrm{v}$ in distilled water) until desired staining intensity was reached (1-3 days). Gels were 
stored in distilled water and transported for subsequent MS analysis at the Erasmus MC Proteomics Center (Rotterdam, The Netherlands). Gel lanes were cut and subjected to in-gel digestion with trypsin (Promega), essentially as described previously. ${ }^{19}$ Polypeptides were identified on an LQT Orbitrap hybrid mass spectrometer (ThermoFischer), coupled to an 1100 series capillary liquid chromatography system (Agilent Technologies) as described elsewhere. ${ }^{20}$ Data analysis was performed using a Mascot based search algorithm (version 2.2; MatrixScience). ${ }^{20}$

\section{Mass spectrometry data analysis}

In order to compare MS results of various samples, all retrieved GI and IPI protein identifiers were converted to Mus Musculus and Homo Sapiens Entrez identifiers, using the Database for Annotation, Visualization, and Integrated Discovery (DAVID), BioDBNet (http://biodbnet.abcc.ncifcrf.gov/db/db2db.php), Clone/Gene ID converter and g:profiler databases. ${ }^{21-24}$ For identifiers that could not be converted to Entrez identifier with these databases, the top hit with matching Entrez ID of a protein blast search (http://blast.ncbi.nlm.nih.gov) using a PSI-Blast algorithm was used. This created one list of all MS identified proteins in our study, which was used for alignment of individual MS sample results using excel software with a DigDB plugin (http://www.digdb.com/). To select true lamin interactors, several selection criteria were sequentially applied. First, proteins needed to be detected at high levels, i.e. average OST-A mascot score above 100, with the average calculated only in OST-A samples in which the protein was detected. Second, proteins were required to be detected at levels minimally 3-fold less in background pull-downs (based on average mascot scores, calculated as described for previous criterion). Third, keratin proteins and proteins with gene ontology (GO) codes for mitochondria and ribosome, which are all considered non-lamin $A$ interacting proteins, were excluded. In order to compare OST-A and OST-P pull-down results in $\mathrm{LMNA}^{-/}$and $\mathrm{LMNA}^{+/+} \mathrm{MEFs}$ these selection criteria were first applied to identify lamina interactors, independent of cell line and OST-A or OST-P construct. Amongst this protein set of lamina interacting proteins, differential interactors were selected by several additional criteria. Proteins were only included if in either cell line the overall average mascot score (including samples in which 
no protein fragment was detected for calculation of the average) of OST-A or OST-P was above 100 and more than 3-fold larger than background pulldown overall average mascot scores. Next, proteins were required to have a ratio of OST-P over OST-A overall average mascot scores above 3.0 or below 0.33 in either $\mathrm{LMNA}^{-/}$or LMNA ${ }^{+/+}$MEFs; . The final inclusion criterion was that this ratio was similar directed (both above or below 1.0) in both cell lines. Mascot scores were considered semi-quantitative only, and were used to distinguish proteins that might either have higher affinity for lamin A or progerin (or associated proteins) or were expressed more abundantly in OST-A versus OST-P cells. Whether these candidates bound lamins directly or indirectly was not revealed by this method.

GO analysis for indicated proteins subsets were performed separately for GO categories "cellular components level 5" (CC), "biological process all" (BP) and "molecular function all" (MF) using Mus Musculus Entrez Identifiers as input values and standard DAVID settings for functional annotation clustering, listing significant clusters by most significant $\mathrm{GO}$ term. ${ }^{22-23} \mathrm{GO}$ analysis was performed against a random protein background consisting of all known Mus Musculus Entrez identifiers, or a set of previously identified nuclear envelope proteins. ${ }^{6,10}$ To identify enrichment of protein complexes a clustering analysis was performed with DAVID against the reactome database, using Homo Sapiens Entrez gene identifiers.

\section{Plasmid transfection}

For verification of differential interactors U2OS Ctrl, OST-A and OST-P expressing cells were plated at $80 \%$ confluency in $10 \mathrm{~mm}$-dishes and FuGENE transfected with previously described expression plasmids for Myc-Nup98, Myc-Nup153, GFP-Nup107, GFP-Tpr and Myc-Prx1 (Kindly provided by respectively Günter Blobel, Brian Burke, Thomas Schwartz, Larry Gerace and Peter Lloyd Jones). ${ }^{25-29} 32$ hours after transfection cells were harvested for a OST pull-down assay.

\section{Western blot}

Western blots were performed essentially as described elsewhere. ${ }^{5}$ SDSPAGE Laemmli sample buffer was added to protein samples from various stages of the OST-pull-down (input, depleted, pull-down) and heated for 20 
minutes at $95^{\circ} \mathrm{C}$ to de-cross-link and denature proteins. Equal amounts of protein extract were loaded and run on NuPage Novex Bis-Tris 4-20\% gradient gels (Invitrogen, Carlsbad, USA), blotted on immobilon-PVDF membrane (Millipore, Billerica, USA), blocked 1 hour in $5 \%$ bovine serum albumin /TBS-T (20 mM Tris- $\mathrm{HCl} \mathrm{pH} \mathrm{7.5,} 150 \mathrm{mM} \mathrm{NaCl}$ and 0.1\% Tween 20). The blocked membrane was incubated at $4^{\circ} \mathrm{C}$ overnight in block buffer diluted primary antibodies. The following antibodies were used: $\alpha$-LaminA/C (Sc6215, Goat, Santa Cruz; detects endogenous Lamin A and C, OST-A, OST-C and OST-P), $\alpha$-LaminA/C (Sc7293, mouse, Santa Cruz; detects only endogenous Lamin A and C, and not OST-A, OST-C or OST-P), $\alpha$-LAP2 (Y-20, Sc19783, Goat, Santa Cruz; detects all LAP2 isoforms), $\alpha$-GFP (Ab6673, Goat, Abcam), $\alpha$-Onestrep (2-1507-001, mouse, IBATagnology), $\alpha$-c-Myc (MS-139$\mathrm{P}$, mouse, Labvision), $\alpha$-Emerin ( $\mathrm{Cl} .4 \mathrm{G} 5$, mouse, Leica), $\alpha$-Beta-Actin (Cl.AC15, A-5441, mouse, Sigma), $\alpha$-Trim28 (A300-767A, rabbit, Bethyl Laboratories), $\alpha$-Ku70 (Ab10878, rabbit, Abcam). After incubation with appropriate secondary HRP-labeled secondary antibodies immunoluminescence was detected with an ECL Western Blotting detection system from Amersham. Intensity of signals were quantified using ImageJ software and statistically analyzed with a Student's t-test.

\section{Results}

\section{Characterization of OneSTrEP tagged A-type lamins}

To ensure protein purification with high affinity and specificity, we made use of a modified version of the well-characterized streptavidin/biotin system. ${ }^{30-}$ ${ }^{31}$ A-type lamins were tagged with the OneSTrEP (OST) tag (Figure S1), and expressed at endogenous levels in human osteosarcoma (U2OS), mouse myocyte NkITAg or mouse embryonic fibroblasts (MEFs) from $\mathrm{LMNA}^{+/+}$or LMNA $^{-1-}$ cells (Figure $1 \mathrm{~A}$ ). ${ }^{14}$ The OST-tag is a biotin analogue, but offers the advantage of binding with high specificity and affinity $\left(K_{d}=1 \mu M\right)$ to the streptavidin analogue Strep-Tactin matrix. ${ }^{18}$ In addition, the OST-tag is relatively small $(3 \mathrm{kDa})$ compared to other epitope tags typically used for MS purposes, thus reducing the likelihood of interference with protein function. To test for physiological functionality of OST-tagged A-type lamins, we first 
A
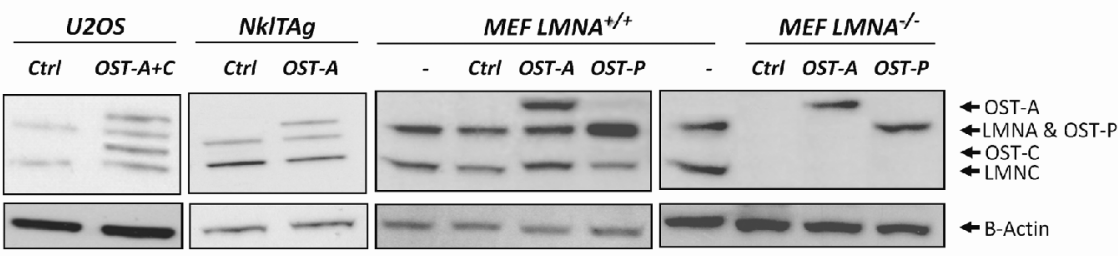

B

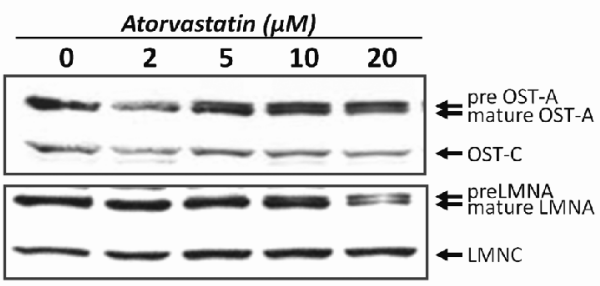

C

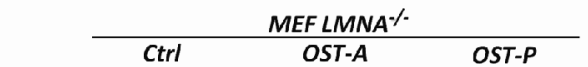

D
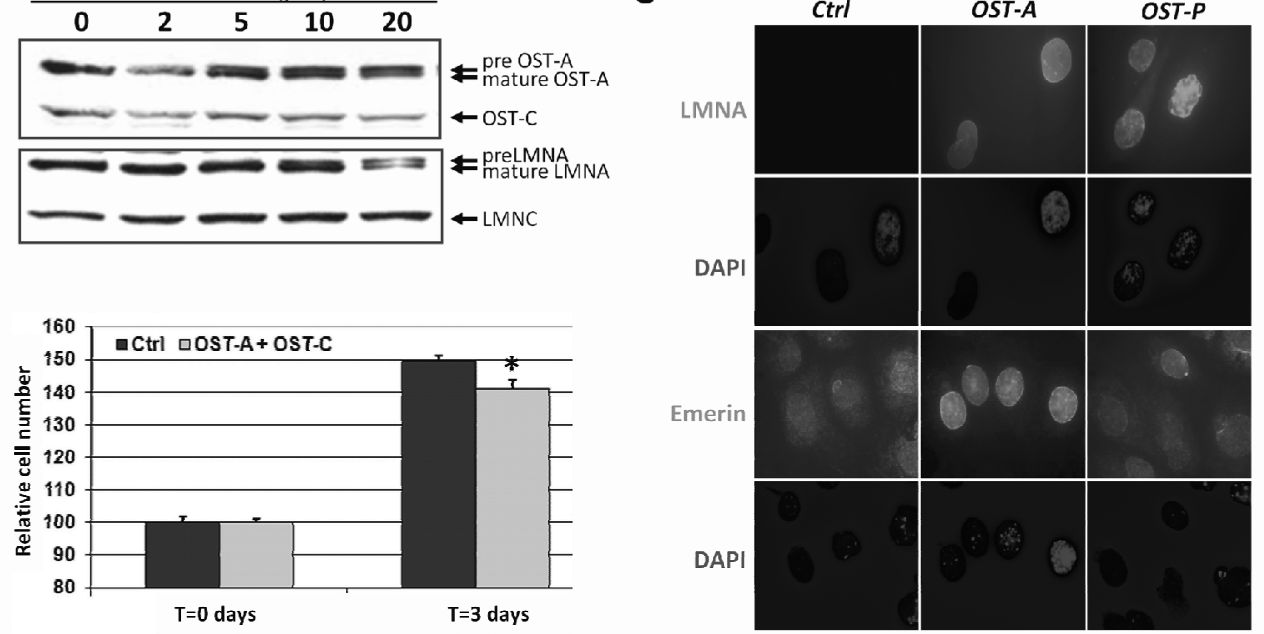

Figure 1. Characterization of OneSTrEP tagged A-type lamins. (A) Western blot of endogenous and tagged A-type lamin in OneSTrEP-tagged lamin A (OST-A), lamin C (OST-C), progerin (OST-P) or control virus-infected mouse cardiac NkITAg myocytes, human osteosarcoma U2OS cells, and mouse embryonic fibroblasts (MEFs) from wildtype (LMNA+/+) or LMNA knock-out (LMNA-/-) embryos. The following antibodies were used: $\alpha$-LaminA/C (Sc6215, Goat, Santa Cruz; detects endogenous Lamin A and C, OST-A, OST-C and OST-P) and $\alpha$-Beta-Actin (Cl.AC15, A-5441, mouse, Sigma). (B) Western blot of endogenous and OST-tagged lamin A, lamin C and prelamin A in U2OS cells infected with OST-A or OST-C and treated for 48 hours with indicated concentrations of atorvastatin, an inhibitor of lamin A farnesylation. The following antibodies were used: $\alpha$-LaminA/C (Sc7293, mouse, Santa Cruz; detects only endogenous Lamin A and C, and not OST-A, OST-C or OST-P) and $\alpha$-Onestrep (2-1507-001, mouse, IBATagnology). (C) Immunofluorescence staining of OST-A, OST-P and control infected LMNA/- MEFs for A-type lamins (LMNA) and emerin. DNA was stained with DAPI. The following antibodies were used: $\alpha$-LMNA/C (Sc-7292, Mouse, Santa Cruz, detects OST-tagged human Lamin A and Progerin, but no endogenous murine lamins), $\alpha$-emerin (Cl.4G5, mouse, Leica). (D) MTS ((3-(4,5dimethylthiazol-2-yl)-5-(3-carboxymethoxyphenyl)-2-(4-sulfophenyl)-2H-tetrazolium)) assay to quantify relative cell number 62 hours after attachment of equal cell numbers of control or OST-A/C infected LMNA-/- MEFs. An asterisk indicates a significant difference $(p<0.05)$ between control and OST-A/C infected cells. 
determined whether OST-tagged lamin A (OST-A) was farnesylated correctly in U2OS cells. Normally mature lamin A is formed after cleavage of the farnesylated C-terminal Caax motif in prelamin by Zmpste24. ${ }^{32}$ Atorvastatin inhibits the synthesis of farnesyl pyrophosphate and thereby impairs farnesylation and processing of prelamin $A$ to mature lamin $A .{ }^{33}$ The farnesylation of both OST-A and endogenous lamin A in U2OS cells was inhibited similarly by increasing concentrations of atorvastatin (Figure 1B). OST-tagged and wildtype lamin $C$ do not contain a Caax motif and remained unaffected by atorvastatin (Figure 1B).

Next, we visualized the localization of OST-A and OST-tagged progerin (OST$\mathrm{P}$ ) in A-type lamin knock-out (LMNA ${ }^{--}$) MEFs (Figure 1C). ${ }^{15}$ As expected, both OST-A and OST-P were detected at the nuclear rim. In contrast to OST-A, OST-P generated distortions in of the nuclear lamina identical to those seen in HGPS patient cells (Figure 1C). ${ }^{16}$ Furthermore, expression of OST-P leads to a decrease of total LAP2 (all isoforms included) and HP1 $p$ protein levels in the nucleus (Figure S2) as observed as well in HGPS and progerin-expressing fibroblast. ${ }^{17}$ OST-A expression further restores correct localization of endogenous emerin in LMNA ${ }^{-/-}$MEFs to the NE (Figure $1 C$ ). ${ }^{34}$ This capacity was reduced after 3 days in culture for OST-P (Figure 1C). Finally, combined expression of OST-A and OST-C inhibited cell proliferation, in line with previously reported endogenous lamin knock-down and HA-tagged lamin A and $C$ overexpression studies (Figure 1D). ${ }^{13}$ Taken together, these data demonstrate that OST-tagged A-type lamins recapitulate endogenous lamin farnesylation, subcellular localization and dominant functions.

\section{OneSTrEP pull-down assay}

To isolate lamin A-associated proteins we used mild reversible cross-linking ( 5 minutes, $1 \%$ formaldehyde) to capture protein/protein and protein/DNA complexes (Figure 2A). Cross-linking enabled us to extensively solubilize Atype lamins by using vigorous sonication in combination with high percentage ionic detergent ( $1 \% \mathrm{SDS}$; Figure 2B). Solubilized protein complexes were diluted to comparable amounts of starting material, by correcting sample volumes to reach equal genomic DNA concentrations, (Figure 2A) and OST-tagged lamins were precipitated using Strep-Tactin matrix, a high-affinity streptavidin analogue. ${ }^{30-31}$ Precipitated lamin-protein 
A

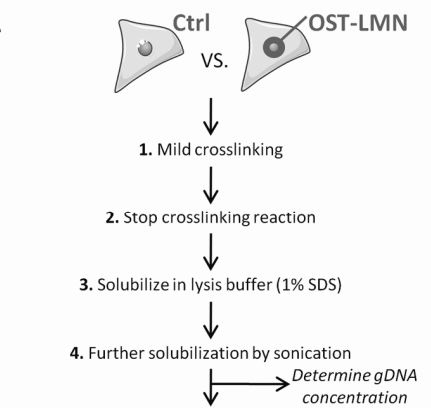

5. Dilute to equal amounts of sample (by EDNA) and $0.1 \%$ SDS final concentration

6. Rotate with Streptactin matrix 7. Stringent washes removing unbound proteins (PULL-DOWN)

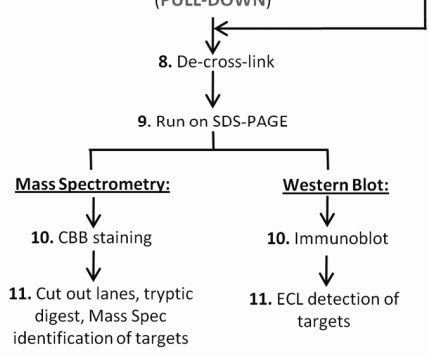

B

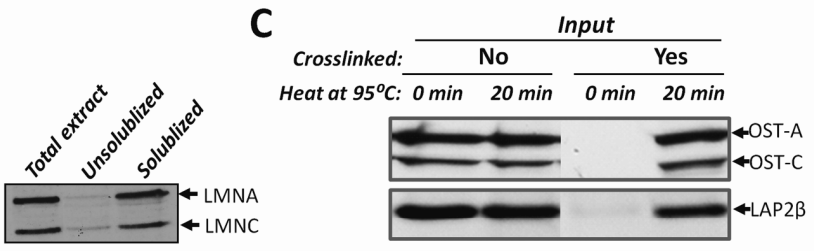

D

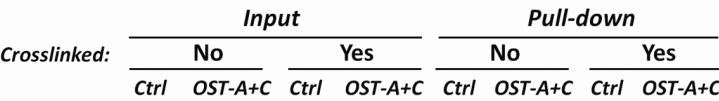

E

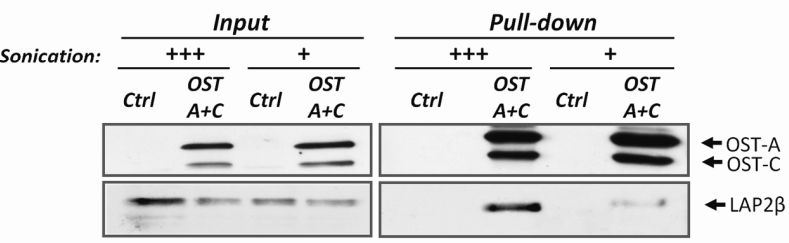

Figure 2. OneSTrEP pull-down assay. (A) Schematic overview of the OST pull-down protocol for cells infected with control and OST-tagged lamin, with the input, depleted and pull-down fraction indicated in bold. Depending on the purpose of the experiment proteins separated on SDS-Page gels were stained with Coomassie Brilliant Blue (CBB) (for mass spectrometry), or immunoblotted for verification of individual candidates. (B) U2OS cells were fixed in 1\% formaldehyde, sonicated in SDS lysis buffer and further diluted in ChIP dilution buffer as described in Materials and Methods. Total protein extracts were spun down, separating the solubilized fraction (i.e. supernatant) and the insoluble fraction (i.e. pellet, twice washed with PBS). Total, unsolubilized and solubilized fractions were compared for endogenous lamin A/C protein levels by western blot. The following antibody was used: $\alpha$-LaminA/C (Sc6215, Goat, Santa Cruz; detects endogenous Lamin A and C). (C) Western blot of U2OS OST-A/C cell extracts ( $1 \%$ formaldehyde vs. no fixation) dissolved in Laemmli sample buffer (no heating vs. 20 minutes at $95^{\circ} \mathrm{C}$ ) before loading on SDS-PAGE gels. The following antibodies were used: $\alpha$-LAP2 (Y-20, Sc19783, Goat, Santa Cruz; LAP2 $\beta$ isoform depicted) and $\alpha$-Onestrep (2-1507-001, mouse, IBATagnology). (D) Western blot of endogenous lamin A/C and Lap2 $\beta$ after cross-linking with $1 \%$ formaldehyde in U2OS cells infected with control or OST-A/C virus. The following antibodies were used: $\alpha$-LAP2 (Y-20, Sc19783, Goat, Santa Cruz; LAP2 $\beta$ isoform depicted), $\alpha$-LaminA/C (Sc7293, mouse, Santa Cruz; detects only endogenous Lamin A and C, and not OST-A or OST-C) and $\alpha$-Onestrep (2-1507001, mouse, IBATagnology). (E) Western blot of OST-pull-down for lamin-A/protein complexes solubilized in SDS lysis buffer with increasing sonication strength (from $12 \times 1 \mathrm{sec}$ at $5 \mu \mathrm{m}$ amplitude, +; to $6 \times 5 \mathrm{sec}$ at $10 \mu \mathrm{m}$ amplitude, +++ ). The following antibodies were used: $\alpha$-LAP2 (Y-20, Sc19783, Goat, Santa Cruz; LAP2 $\beta$ isoform depicted) and $\alpha$-Onestrep (2-1507-001, mouse, IBATagnology). 
epitope masking on western blot without detectable protein degradation (Figure 2C). Cross-linking was crucial for co-precipitation of lamin complexes were eluted and de-crosslinked under conditions $\left(20\right.$ minutes at $95^{\circ} \mathrm{C}$ in Laemmli Sample Buffer) that fully reversed crosslink-induced interactors, as omitting this step resulted in loss of the known OST-A/C interaction partners wildtype lamin A/C and LAP2 $\beta$ in purified protein complexes (Figure 2D). ${ }^{35-36}$ Increasing sonication strength drastically improved retrieval of the lamin $A$ interacting partner LAP2 $\beta$ (Figure 2E). These findings demonstrate that the OST-tag/Strep-Tactin pull-down approach is able to recover lamin A protein complexes after temporal cross-linking and solubilization.

\section{Identification of lamin A interacting proteins}

To identify and characterize lamin A-interacting proteins OST pull-downs were performed on murine cardiac myocyte NkITAg cells expressing OST-A at endogenous levels or a control virus lacking the lamin A transgene. Copurified proteins were size-separated and retrieved from SDS-PAGE gels and analyzed by MS (Figure 3A). In total 5,162 unique peptide fragments corresponding to 649 individual proteins were detected in two independent experiments (Figure $3 \mathrm{~A}$ ). We reduced the list of potential interactors by stringent restrictive analysis (i.e. mascot score cut-off, minimum fold over background and filtering out non-essential peptides; see Materials and Methods; Figure $3 A$ ) to 60 proteins. The set of identified proteins contains several known lamin A interactors including Lamin B, Lap2B ,Emerin, MAN1, Sun1 and 2, as well as the muscle specific interactor Syne-2 (Figure 3A, Table S1). ${ }^{37}$ About half of our candidate lamin A interactors were identified in two previous NE proteome studies which had identified proteins in an INM/lumen/ONM ${ }^{6}$ fraction or an INM/lamina/nucleoplasmic fraction ${ }^{10}$, respectively, in other cell types. ${ }^{6,10}$

We identified 29 novel lamin A interactors (Table S1). These include proteins implicated in muscle differentiation (Luc1-like, Serpine1 mRNA binding protein), transcription factors (Strap, Similar to calponin 3), DNA repair (Ku70) and heterochromatin maintenance (Trim28). ${ }^{38-43}$ Several known (Emerin, Lap26) as well as previously unidentified lamin A interactors (Trim28, Ku70) were chosen for validation of the MS data by targeted coprecipitation. Trim 28 and $K u 70$ were specifically chosen as they represented 
A

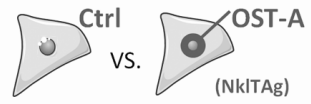

649 proteins detected $(100 \%)$

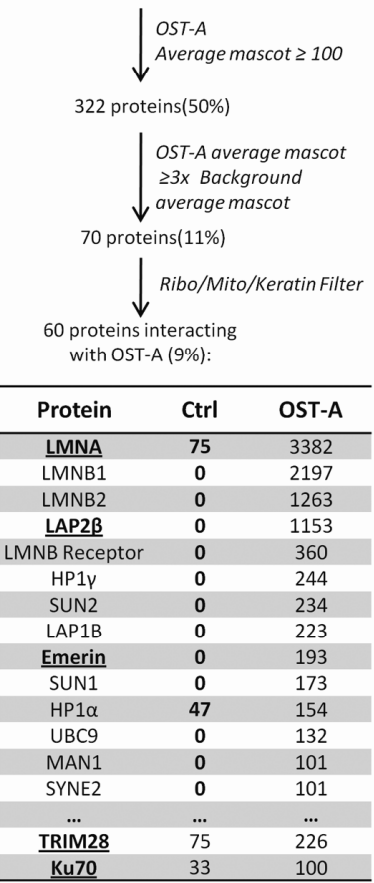

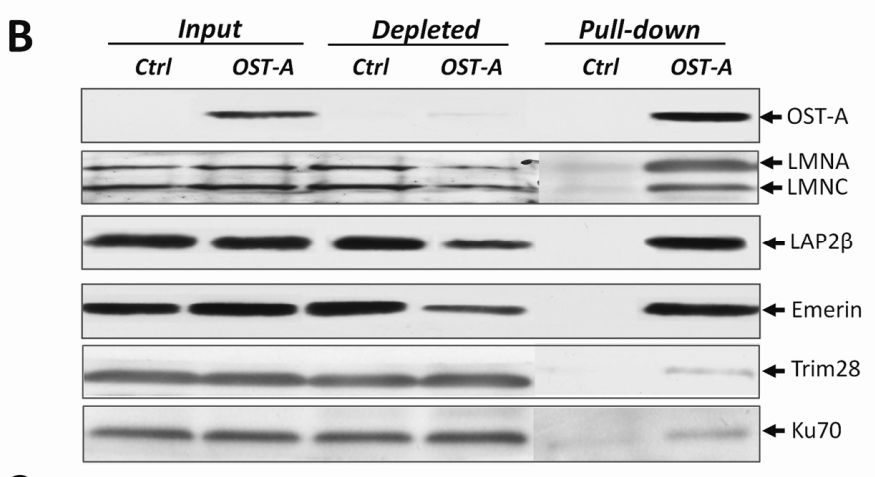

C

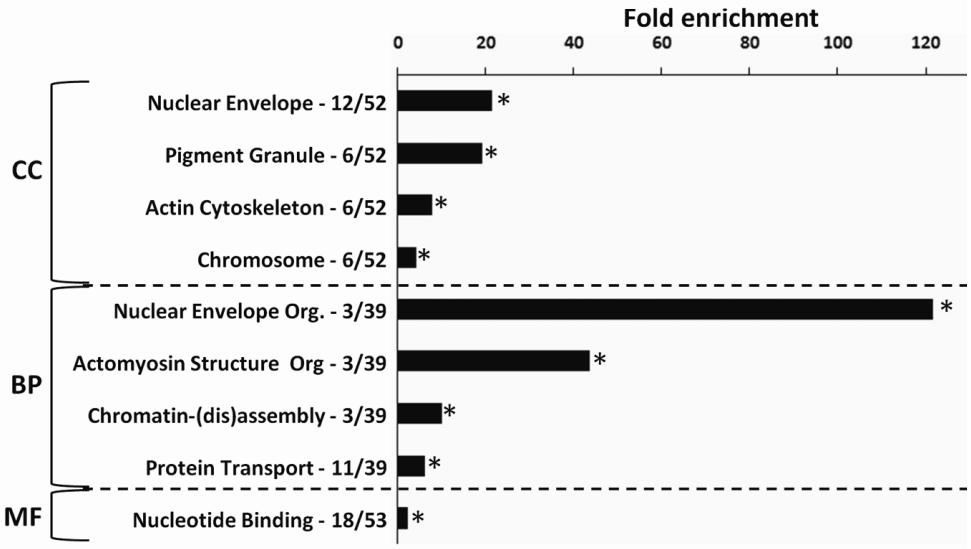

Figure 3. Identification of lamin A interacting proteins. (A) Overview of the selection criteria for identification of lamin A interacting proteins from raw mass spectrometry data. Control and OST-A pulldown average mascot scores for several lamin A interactors identified in NkITAg cells are depicted (Underlined proteins are verified in figure 3B). (B) Western blot indicating protein levels of mass spectrometry identified lamin A-interactors (endogenous lamin A/C, Lap2B, Emerin, Trim28 and Ku70) precipitated in NkITAg cells infected with control or OST-A virus. The following antibodies were used: $\alpha$ Onestrep (2-1507-001, mouse, IBATagnology), $\alpha$-LaminA/C (Sc7293, mouse, Santa Cruz; detects only endogenous Lamin A and C, and not OST-A), $\alpha$-LAP2 (Y-20, Sc19783, Goat, Santa Cruz; LAP2 $\beta$ isoform depicted), $\alpha$-Emerin (Cl.4G5, mouse, Leica), $\alpha$-Trim28 (A300-767A, rabbit, Bethyl Laboratories) and $\alpha$-Ku70 (Ab10878, rabbit, Abcam). (C) GO analysis of identified lamin A interactors. GO analysis was performed separately for the GO categories "cellular components" (CC), "biological process" (BP), "molecular function" (MF) and compared against a random protein set. ${ }^{22-23}$ The number of lamin A interacting proteins identified within a specific $\mathrm{GO}$ category versus the total amount of annotated lamin $\mathrm{A}$ interacting proteins is indicated next to the significantly enriched GO categories $\left(p<1 \times 10^{-3}\right.$ indicated by asterisk; statistical tests include using a kappa statistic measurement and is further described elsewhere). ${ }^{71}$ 
the two proteins that were closest to the selection cut-off and therefore were most likely to represent false positives. OST-A co-precipitated considerable amounts of endogenous Lamin A/C, Lap2B, Emerin, as well as Trim 28 and $K u 70$ (Figure $3 B$ ). In contrast no interactions were observed in control pull-downs from cells only expressing OST (Figure 3B). In comparison to Lap2B and Emerin, Ku70 and Trim28 appear to be weaker interactors of OST-A (Figure $3 B$ ). In addition to Lap2B we identified interactions of lamin A with Lap $2 \alpha, \gamma, \delta$ and $\varepsilon$ isoforms in U2OS cells by OST pull-down and immunoblotting (Figure S3). Taken together these data demonstrate that the OST pull-down/MS assay and bioinformatical selection criteria can be used for identification of lamin A interacting proteins.

\section{Functional annotation of lamin A interacting proteins}

To globally characterize the identified lamin A interactors, we performed a $\mathrm{GO}$ analysis to describe the subcellular localization (CC, cellular component), biological processes (BP) and molecular functions (MF) of identified interactors (Figure $3 \mathrm{C}$ ). As expected, compared to random proteins lamin A interactors were 22-fold enriched for 'nuclear envelope' components $\left(p=5.0 \times 10^{-12}\right.$; Figure 3C, TableS2), and to a lesser extent for other subcellular compartments. The interactors are enriched in the functional groups of NE organization' $\left(p=2.5 \times 10^{-4}\right)$, actomyosin structure organization $\left(p=9.9 \times 10^{-3}\right)$, and protein transport $\left(p=5.6 \times 10^{-6}\right)$, with a preferential molecular function of 'nucleotide binding' $\left(p=5.5 \times 10^{-4}\right)$. The GO analysis had high similarity to the previously identified proteins in the NE proteome (Table S2). ${ }^{6,10}$

\section{Identification of preferential lamin A or progerin interactors}

We next applied the OST pull-down method to identify proteins that bind exclusively to lamin A or progerin as well as interactors that bind to both proteins with different affinity. To this end, we expressed control virus, OSTA or OST-P in $\mathrm{LMNA}^{-/}$or $\mathrm{LMNA}^{+/+}$MEFs at endogenous levels (Figure 1A). OST pull-downs were performed in duplicate in all cell lines. In total we detected 54,939 unique peptide fragments in all OST and control pulldowns, corresponding to 2804 different proteins (Figure 4A). Using similar selection criteria as validated for the NkITAg OST-A pull-down (mascot score 
A

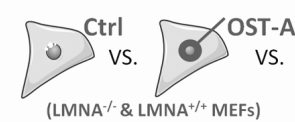

2804 proteins detected $(100 \%)$

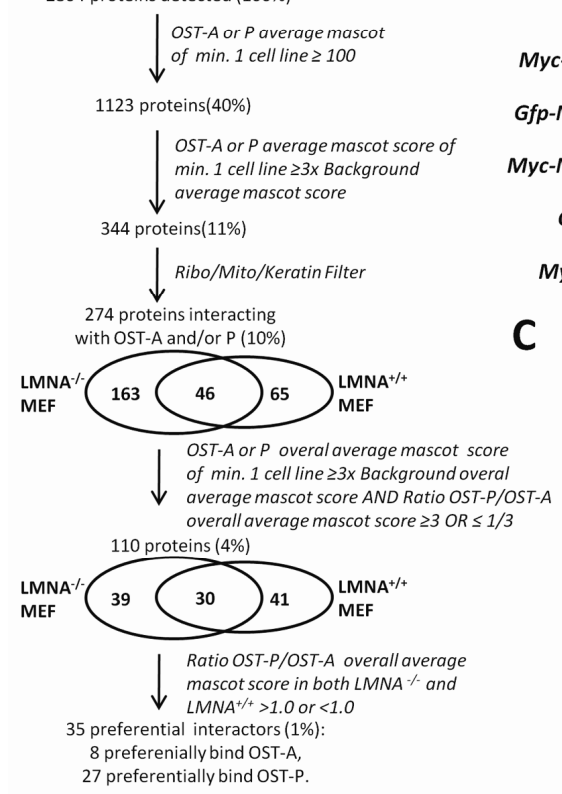

B

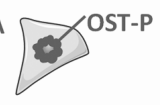

C
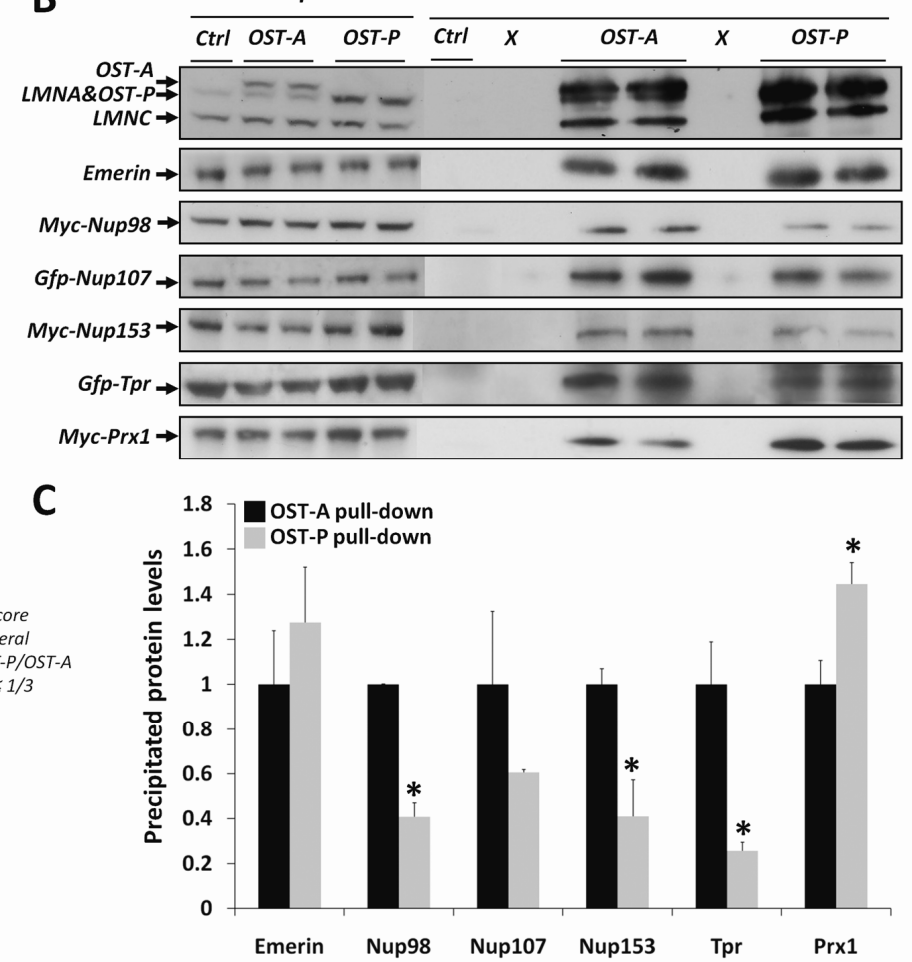

Figure 4. Identification of preferential lamin A/progerin interacting proteins. (A) Overview of the selection criteria applied to identify preferential laminA/progerin interacting proteins (See also Material and Methods). (B) Verification of lamin A and progerin preferential interactors in U2OS cells transfected with GFP-Nup107, GFP-Tpr, Myc-Nup98, Myc-Nup153 or Myc-Prx1. OST pull-down was performed in U2OS cells infected with control, OST-A or OST-P virus. The following antibodies were used: $\alpha$-LaminA/C (Sc6215, Goat, Santa Cruz; detects endogenous Lamin A and C, OST-A, and OST-P), $\alpha$-Emerin (Cl.4G5, mouse, Leica), $\alpha-C$ Myc (MS-139-P, mouse, Labvision) and $\alpha$-GFP (Ab6673, Goat, Abcam). (C) Quantification of western blot protein levels in OST-A and OST-P pull-down fractions (average \pm standard deviation) corrected for input signal intensity. Relative protein levels for OST-A pull-down fractions were set arbitrarily at 1 . Protein levels identified by western blot in OST-P pull-down fractions (Figure 4B) are indicated. Asterisks indicate a significant ( $p<0.05$; Student's T test) difference between OST-A and OST-P pull-down fraction. 
cut-off, minimum fold over background and filtering out non-essential peptides; see Materials and Methods) we defined 274 lamina-interacting proteins, 209 in $\mathrm{LMNA}^{-/-}$MEFs and 111 in $\mathrm{LMNA}^{+/+}$MEFs, of which 46 proteins were identified as interactors in both cell lines. Of these 274 interactors 150 proteins interacted with lamin A and 170 with progerin (Table S5). The relatively low number of interactors identified in both $\mathrm{LMNA}^{+/+}$and ${ }^{\%}$ cells may be due to the very high stringency used in identifying interactors or may be due to differential effects of lamin A expression in a wild-type or null background. As many of the identified lamin A interactors were also detected by MS at levels just below inclusion criteria in progerin pull-down samples and vice versa, we used additional criteria to identify preferential interactors (minimal fold difference between OST-A and OST-P mascot scores and similarity of results in $\mathrm{MEFs}^{-1-}$ and $\mathrm{MEFs}^{+/+}$; see Materials and Methods; Figure 4A). 110 proteins interacted with both lamin $A$ and progerin and applying these criteria resulted in a final selection of 35 proteins candidates that preferentially interact with either wildtype lamin A (8 candidates, including Nup153, Nup98 and Translocated promoter region) or progerin (27 candidates, including Paired related homeobox 1 (Prx1), Proliferating cell nuclear antigen (Pcna) and Pinin).

To validate preferential interactors we performed OST pull-downs in U2OS cells expressing control virus, OST-A or OST-P at endogenous levels (Figure $4 B$ ) and transiently expressed several potential differential interactors (Myctagged Nup98, Nup153, Prx1 and GFP-tagged Tpr). In support of the pulldown results, we found weaker interaction of OST-P with Nup98, Nup153 and $\operatorname{Tpr}$ (all $\mathrm{p}<0.05$, Figure 4B and C). The NPC protein Nup107 also showed reduced, albeit statistically not significant, binding to OST-P. Prx1 was confirmed to have increased, but not unique, affinity for OST-P $(p<0.05$, Figure $4 \mathrm{~B}$ and $\mathrm{C}$ ). Finally, immunoblot detection confirmed the predicted interaction of endogenous emerin with OST-tagged lamin $A$ and progerin ( $p>0.05$, Figure $4 B$ and $C$ ). These data show that lamin $A$ and progerin have distinct protein-interactions that can be identified by OST pull-down assays.

\section{Functional analysis of preferential lamin A or progerin interactors}

A GO-signature comparison of 150 identified lamin A-associated proteins and 170 progerin-associated proteins, shows that both protein sets were 
Table 1. Overview of preferential lamin A/progerin interactors.

\begin{tabular}{|c|c|c|c|c|c|c|c|c|c|c|}
\hline & $\begin{array}{l}\text { Entrez } \\
\text { M.M. }\end{array}$ & Abbrevation & Name & 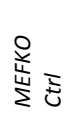 & 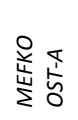 & 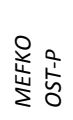 & 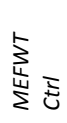 & $\sum_{\substack{L \\
\sum}}^{\frac{T}{n}}$ & $\begin{array}{l}5 \\
\text { 离京 } \\
\sum\end{array}$ & 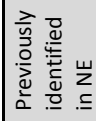 \\
\hline \multirow{27}{*}{ 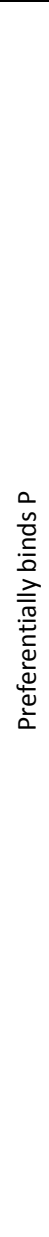 } & 21825 & Thbs1 & Thrombospondin 1\# & 133 & 0 & 627 & 0 & 0 & 21 & No \\
\hline & 22004 & Tpm2 & Tropomyosin 2, beta & 0 & 0 & 219 & 249 & 199 & 290 & No \\
\hline & 634386 & FTL2 & Ferritin light chain 2\# & 0 & 0 & 158 & 0 & 0 & 35 & No \\
\hline & 14156 & Fen1 & $\begin{array}{l}\text { Flap structure specific } \\
\text { endonuclease } 1\end{array}$ & 0 & 0 & 156 & 57 & 118 & 172 & No \\
\hline & 19072 & Prep & Prolyl endopeptidase & 32 & 0 & 130 & 99 & 58 & 140 & No \\
\hline & 665766 & PSME1 & Proteasome 28 subunit, alpha & 0 & 0 & 123 & 20 & 0 & 92 & No \\
\hline & 17524 & Mpp1 & Membrane protein, palmitoylated & 0 & 0 & 111 & 0 & 0 & 29 & No \\
\hline & 241490 & Rbm45 & RNA binding motif protein 45 & 55 & 0 & 62 & 0 & 0 & 122 & No \\
\hline & 67543 & Pabpc3 & $\begin{array}{l}\text { Poly(A) binding protein, } \\
\text { cytoplasmic } 3\end{array}$ & 0 & 0 & 51 & 0 & 0 & 276 & No \\
\hline & 18949 & Pnn & Pinin & 299 & 0 & 49 & 0 & 0 & 105 & Yes $^{10}$ \\
\hline & 18933 & Prx1 & Paired related homeobox 1 & 0 & 0 & 28 & 0 & 0 & 135 & No \\
\hline & 108123 & Napg & $\begin{array}{l}\mathrm{N} \text {-ethylmaleimide sensitive fusion } \\
\text { protein attachment protein } \\
\text { gamma }\end{array}$ & 0 & 15 & 145 & 0 & 0 & 23 & No \\
\hline & 18950 & Pnp & $\begin{array}{c}\text { Similar to purine nucleoside } \\
\text { phosphorylase; purine-nucleoside } \\
\text { phosphorylase } 1\end{array}$ & 18 & 20 & 168 & 0 & 0 & 57 & No \\
\hline & 433862 & Ppid & Peptidylprolyl isomerase D & 61 & 26 & 189 & 0 & 96 & 153 & Yes $^{10}$ \\
\hline & 74122 & Tmem43 & Transmembrane protein 43 & 0 & 27 & 181 & 0 & 165 & 172 & Yes $^{6}$ \\
\hline & 110960 & Tars & Threonyl-tRNA synthetase & 18 & 61 & 295 & 16 & 0 & 99 & No \\
\hline & 15525 & Hspa4 & Heat shock protein 4 & 100 & 92 & 334 & 16 & 49 & 60 & No \\
\hline & 14300 & Frg1 & FSHD region gene 1 & 46 & 41 & 138 & 0 & 62 & 64 & No \\
\hline & 18538 & Pcna & Proliferating cell nuclear antigen & 0 & 58 & 189 & 0 & 54 & 101 & No \\
\hline & 28114 & Nsun2 & Sun domain family member 2 & 56 & 57 & 182 & 99 & 77 & 174 & No \\
\hline & 13722 & Scye1 & $\begin{array}{l}\text { Aminoacyl tRNA synthetase } \\
\text { complex-interacting } \\
\text { multifunctional protein } 1\end{array}$ & 0 & 105 & 199 & 24 & 29 & 152 & No \\
\hline & 70804 & Pgrmc2 & $\begin{array}{l}\text { Progesterone receptor membrane } \\
\text { component } 2\end{array}$ & 0 & 320 & 603 & 0 & 0 & 125 & Yes $^{6}$ \\
\hline & 12903 & Crabp1 & $\begin{array}{l}\text { Cellular retinoic acid binding } \\
\text { protein I }\end{array}$ & 128 & 198 & 361 & 68 & 35 & 279 & No \\
\hline & 14683 & Gnas & $\begin{array}{l}\text { Guanine nucleotide binding } \\
\text { protein, alpha stimulating }\end{array}$ & 19 & 106 & 182 & 0 & 0 & 100 & No \\
\hline & 19344 & Rab5b & RAB5B & 0 & 289 & 350 & 0 & 0 & 133 & Yes $^{10}$ \\
\hline & 620016 & LOC620016 & Glutaredoxin 3 & 19 & 41 & 44 & 19 & 28 & 130 & No \\
\hline & 65114 & Vps35 & Vacuolar protein sorting 35 & 239 & 432 & 457 & 142 & 131 & 447 & No \\
\hline \multirow{8}{*}{ 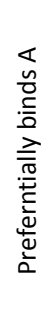 } & 56758 & Mbnl1 & Muscleblind-like 1 & 0 & 38 & 17 & 0 & 104 & 0 & No \\
\hline & 218210 & Nup153 & Nucleoporin 153 & 0 & 302 & 101 & 0 & 218 & 141 & Yes ${ }^{6,10}$ \\
\hline & 109168 & 5730596K20Rik & Atlastin GTPase 3 & 104 & 360 & 89 & 0 & 75 & 50 & No \\
\hline & 226747 & Ahctf1 & $\begin{array}{l}\text { AT hook containing transcription } \\
\text { factor } 1\end{array}$ & 0 & 143 & 32 & 0 & 47 & 26 & No \\
\hline & 108989 & Tpr & Translocated promoter Region & 0 & 234 & 42 & 0 & 268 & 133 & Yes $^{10}$ \\
\hline & 270192 & Rab6b & RAB6B & 0 & 101 & 0 & 0 & 180 & 0 & No \\
\hline & 269966 & Nup98 & Nucleoporin 98 & 0 & 178 & 0 & 0 & 227 & 125 & Yes $^{6,10}$ \\
\hline & 14681 & Gnao1 & $\begin{array}{c}\text { Guanine nucleotide binding } \\
\text { protein, alpha } \mathrm{O}\end{array}$ & 0 & 192 & 0 & 0 & 43 & 0 & No \\
\hline
\end{tabular}

Preferential lamin A/progerin interacting proteins were identified amongst all lamin A and progerin interactors in $\mathrm{LMNA}^{-1-}$ and $\mathrm{LMNA}^{+++} \mathrm{MEFs}$ according to standard selection criteria (see Materials and Methods). Overall average mascot scores for individual pull-downs are indicated, as well as whether proteins were identified in two previous NE proteome studies (see grey shaded column) which had identified proteins in an INM/lumen/ONM ${ }^{6}$ fraction or an INM/lamina/nucleoplasmic fraction ${ }^{10}$. Hashes indicate proteins GO-annotated as 'Extracellular region'. 
over 50-fold enriched for INM and nuclear lamina proteins, are preferentially involved in NE organization, mRNA and protein transport and enriched for proteins with GTP binding capacity (Figure S4, Table S3). Lamin A interactors were preferentially enriched $\left(p<1 \times 10^{-4}\right)$ in NPC-related nucleocytoplasmic and protein transport proteins and to a lesser extent $\left(1 \times 10^{-2}<p>1 \times 10^{-3}\right)$ in proteins of the NE, the cytoskeleton and ECM ('sarcomere' and 'PDGF binding' GO-terms). Progerin interactors showed enrichment for endoplasmatic reticulum (ER) membrane proteins involved in protein folding and processing ('cell redox homeostasis', 'intramolecular oxidoreductase' and 'signal peptidase processing' GO-terms) as well as proteins involved in chromatin organization (mainly histone 1 and $2 \mathrm{~A}$ variants). ER-localized A-type lamin interacting proteins were preferentially membrane-associated (43 out of 46) and not lumenal localized ( 3 out of 46). To further assess the functional context of lamin $A$ and progerin interactors we used the Reactome protein interaction database, which contains maps of known protein complexes, as a reference. ${ }^{44}$ This analysis showed that 10 proteins of the $26 \mathrm{~S}$ proteasome complex were identified as progerin interactors (Figure S4, Table S3). These GO and Reactome profiles for lamin $A$ and progerin interactors resemble those found in the previously identified NE proteome (Table S3).

GO-analysis of the 35 preferential lamin A/progerin interactors showed enrichment of NE proteins, proteins involved in RNA metabolism ('ncRNA metabolic transport' and 'RNA metabolic process' GO terms), general protein transport components, including members of the NPC ('Ran binding protein 2' Reactome term) and proteins with GTPase activity (All $p<1 \times 10^{-2}$; Figure 5A, 5B, Table S2). ${ }^{44}$ NPC lamin-interacting proteins were preferentially located at the nucleoplasmic site of the NPC (Figure 5C), of which a vast majority showed an overall diminished interaction with progerin in both $\mathrm{LMNA}^{-/}$and $\mathrm{LMNA}^{+/+} \mathrm{MEFs}$, including 3 proteins identified in our assay as preferential lamin A interactors (Tpr, Nup98, Nup153; Figure 5C, Table 1). 


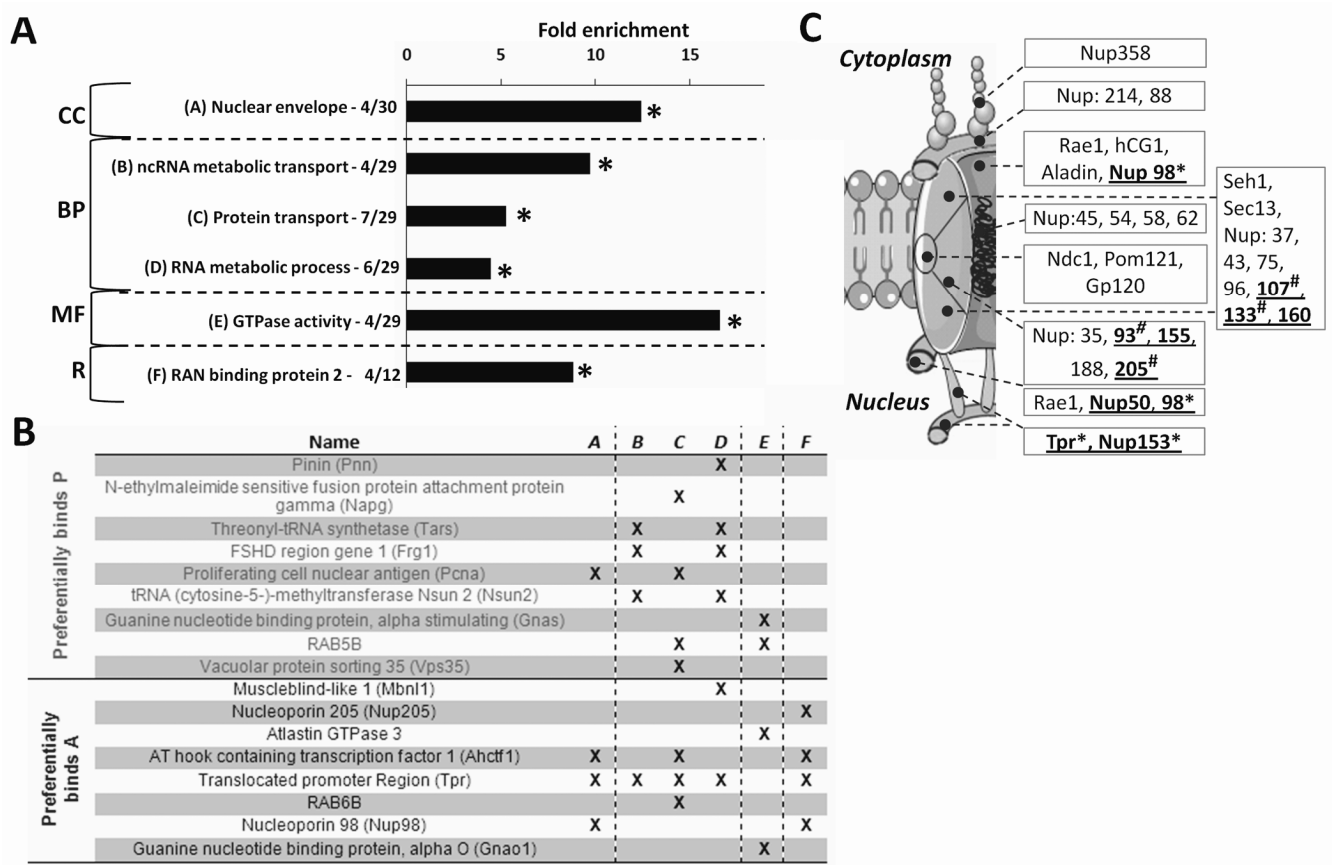

Figure 5. Functional annotation of preferential lamin A/progerin interactors. (A) GO profile of preferential interactors specified for "cellular components" (CC), "biological process" (BP), "molecular function" (MF) GO categories. Representation of protein complexes was analyzed using the Reactome database $(R)^{44}$. Significant categories based on comparision are indicated by asterisks $\left(p<1 \times 10^{-3}\right.$; random proteins used as background; statistical tests include a kappa statistic measurement as described elsewhere ${ }^{71}$ ), as well as the number of proteins within the specific category out of the total number of annotated preferential interactors. (B) Overview of $\mathrm{GO}$ annotated lamin A/progerin preferential interactors in significant categories. Individual categories are marked $A-F$ and correspond to figure 5A. (C) Schematic illustration of the nuclear pore complex (NPC) indicating the location of lamin A-interacting NPC proteins identified by mass spectrometry analysis (underlined). ${ }^{72}$ Identified interactors preferentially localize in the nuclear basket (Nup153, Tpr), nuclear ring (Nup50, Nup98) or scaffold (Nup-93, $107,-133,-155,-160$ and -205$)$. Nup98 has been described to localize in both the nuclear basket as well as the NPC central channel. Asterisks indicate proteins identified to preferentially interact with lamin A. Hashes indicate proteins with MS data indicative of diminished interaction with progerin as well (similar direction change for ratio OST-P/OST-A overall average mascot ratio's in both MEF LMNA ${ }^{-/}$and MEF $\operatorname{LMNA}^{+/+}$). 


\section{Discussion}

The systematic mapping of lamin A interaction partners is currently hampered by the insoluble nature of lamina proteins. We have developed and applied a novel pull-down approach based on the One-STrEP tag to identify in vivo A-type lamin interactors, of which a subset interacts preferentially with lamin A compared to its mutant isoform progerin. To isolate lamin-associated protein complexes we took advantage of the OneSTrEP-tag/Strep-Tactin matrix system, an engineered version of the strongest non-covalent biological interaction known (biotin/streptavidin). ${ }^{18}$, ${ }^{45}$ We first verified that the addition of a $3 \mathrm{kDa}$ small OST-tag to lamin A recapitulated endogenous lamin $A$ as judged by farnesylation, subnuclear localization, emerin NE localization and cell proliferative effects. Lamin interactors were identified, by subsequent solubilization of the majority of A-type lamins, high affinity precipitation of OST-tagged lamins, high stringency washes of precipitated protein complexes, important as lamins have a propensity to non-specifically interact with other proteins, and a highly restrictive selection based on MS results. ${ }^{46}$ We identify a large number of known direct A-type lamin interactors (including Emerin, Ubc9, $L A P 2 \alpha, L A P 16, L M N B 1, R b b p 7)$, as well as known indirect interactors (including Nesprin/Syne-1, Nesprin/Syne-2; HP1 $/$ /CBX3, HP1 $\alpha / C B X 5, M A N 1$ ), therefore directly validating our approach. ${ }^{5,12,47-52}$ Our approach is able to identify physical associations which occur via multiple hierarchical interactions as indicated by the identification TorsinA, which is indirectly connected to the lamina via Nesprins and Sun proteins. ${ }^{52}$ The identification and verification of two previously unidentified lamin A interactors (Trim28 and $K u 70$ ) that met the inclusion criteria justifies the applied selection threshold. Comparative analysis with two published NE proteomes further validates the OST approach. ${ }^{6,10}$ Although these studies differ in their starting materials with one using isolated nuclear envelopes and the other isolated lamina preparations, they identify a significant part of the NE proteome, including laminar proteins, and therefore are considered of value for a cross-comparison. About half of the OST pull-down identified lamin A interactors in NkITAg cells were identified in these previous studies and the GO signature of our hits highly resembles that of NE proteins identified in these analyses. 6,10 
Our OST pull-down method differs from previously published approaches. First, in comparison to approaches that biochemically fractionate nuclear membranes or the lamina, we specifically precipitate lamin A interactors, regardless of their subcellular localization. Secondly, the inclusion of a crosslinking step preserves short-lived lamin-protein interactions and thereby contributes to detection of identified transient and indirect interactors. Thirdly, we use a highly sensitive and stringent MS analysis approach. Our validation indicates a low level of false positives. However, we cannot formally rule out that a small percentage of identified hits reflect nonspecific interactions. Especially various extracellular matrix (ECM) identified interactors and proteins involved in Golgi related vesicle trafficking might reflect non-specific interactions. ER proteins form a group of identified interactors that have been considered background in previous NE proteomic studies. ${ }^{10}$ Although this exclusion is correct for lumenal ER proteins, which are not directly accessible to lamins, membrane-associated ER proteins could reflect bona fide interactors of OST-tagged lamins involved in protein folding and processing during their synthesis and post-translational modification or diffuse from the ER to the continuous INM and anchor to the lamina. ${ }^{53}$ In accordance with this, our data indicate that almost all identified A-type lamin interactors residing in the ER are membraneassociated and not lumenal.

Our method combines beneficial aspects of several methods used to identify protein-content of and interactions at the nuclear lamina. IP studies have the advantage of enriching for the interactome of a protein of interest and the ability to analyze the impact of mutations on protein interactions, but are hampered by the need to solubilize intact NE protein complexes. ${ }^{54}$ In contrast, differential extraction from isolated whole nuclear membrane fractions permits disruption of protein complexes prior to MS analysis, but identifies general protein composition at a subnuclear level based on similarity of proteins in biochemical characteristics. ${ }^{6,8}{ }^{8} 10$ Yeast two-hybrid screens allow comparison of protein interactions for wild-type and mutated protein fragments without solubilization, but have the drawback of high false positive-rates and non-physiological conditions. ${ }^{55-57}$ The OST pull-down approach combines advantages of identifying and comparing the interactome for a wild-type and mutated protein of interest with a low false positive rate under physiological conditions, with complete extraction of 
intact lamina protein complexes. These unique characteristics complement current technology to identify lamin A interactors.

We identified both interactors of lamin A and of progerin and compared their GO profiles. Differences in profiles indicate a loss of interactions of progerin with the cytoskeleton in comparison to lamin A, in agreement with reported disruption of nuclear-cytoskeletal coupling by progerin. ${ }^{58}$ Furthermore, GO analysis suggests a preferential interaction of progerin with protein folding and processing enzymes as well as proteasome complex members. This observation is intriguing in the light of the reported degradation in HGPS patient cells of a large set of nuclear proteins, particularly chromatin-associated proteins., 16 To further investigate differences between lamin A- and progerin-interacting proteins we identified preferential interactors by comparing OST pull-downs in both LMNA $^{-/-}$MEFs, in the absence of endogenous lamin A/C which could mask distinct interactions due to dimerization with progerin, as well as biological more relevant $\mathrm{LMNA}^{+/+}$MEFs. ${ }^{36}$ Several identified distinct lamin A/progerin interactors preferentially possessed GTPase activity and others were involved in RNA metabolism or nucleocytoplasmic transport.

We found reduced interaction of Nup153 with progerin compared to wildtype lamin A. Nup153 anchors NPCs to the nuclear lamina and directly interacts with $T p r$, which is involved in formation of NPC-associated heterochromatin exclusion zones. ${ }^{59-61}$ Interestingly, HGPS is associated with peripheral loss of heterochromatin and clustering of Nup153 and other NPC proteins. ${ }^{62-63} \mathrm{~A}$ diminished interaction between OST-P and Nup153 could explain a loss of NPC anchoring to the nuclear lamina, as well as clustering and defective NPC-mediated nuclear import which have all been implicated in HGPS. ${ }^{62-63}$

We found an increased interaction of progerin with paired related homeobox 1 (Prx1 or Prrx1), a transcriptional co-activator involved in skeletogenesis and vasculogenesis. ${ }^{28,64}$ Loss of Prx1 results in lethal vertebrae and craniofacial skeletal defects, while overexpression induces vascular smooth muscle cell (VSMC) proliferation and ECM remodeling by direct upregulation of tenascin $C$ expression. ${ }^{28,}{ }^{64}$ These phenotypes highly resemble HGPS etiology as Zmpste24 knock-out mice, which serve as a 
model system for HGPS, suffer from osteogenesis imperfecta, manifested in vertebrae and craniofacial bone fractures. ${ }^{65}$ Furthermore progerin drastically downregulates tenascin $C$ expression and progerin transgenic mice suffer from a progressive loss of VSMCs and changes in ECM composition. ${ }^{66-67}$ An increased interaction of progerin with Prx1 could impair transcriptional activation of tenascin $C$ and cause consecutive HGPS characteristic ECM, VSCM and bone related disorders.

A number of differential interactors link lamin A and progerin to previously reported abnormal cell biology in the context of $L M N A$ mutations. We find an increased interaction of Proliferating cellular nuclear antigen (Pcna) with progerin. Pcna, as a replication fork processivity factor, regulates genomic replication and has been reported to redistribute upon introduction of progerin. ${ }^{68}$. Several studies report abnormal proliferation in LMNA mutant cells. ${ }^{69}$ Pcna is thought to become trapped in intranuclear mutant lamin aggregates, thereby ultimately blocking DNA replication and causing HGPSassociated DNA damage. ${ }^{63,68}$ Of relevance in this context is also the identification of Trim28 and Ku70 as interaction partners of lamin A. Both are associated with DNA damage response and/or repair and Trim28 is a reported interaction partner of heterochromatin protein $1 .^{70}$ While the contribution of these preferential interactions will need to be elucidated in targeted studies, the global identification of novel lamin A and progerin interactors using a novel pull-down method has generated several leads for studies of specific interaction partners of lamin A and its mutants with the goal of uncovering the molecular mechanisms of tissue-specific laminopathies.

\section{Acknowledgements}

We thank Günter Blobel, Brian Burke, Thomas Schwartz, Larry Gerace and Peter Lloyd Jones for kindly sharing expression plasmids. Thijs Konsten is thanked for his help in optimization of the OST pull-down assay. This research was supported in part by the Intramural Research Program of the National Institutes of Health $(\mathrm{NIH}), \mathrm{NCl}$, Center for Cancer Research, grants from the Dutch Heart Foundation, ZonMW and the EU-KP7 grant 'Inheritance'. 


\section{References}

1. Foisner R. Dynamic Connections of Nuclear Envelope Proteins to Chromatin and the Nuclear Matrix. In: Collas P, ed. Nuclear Envelope Dynamics in Embryos and Somatic Cells, 2003.

2. Broers JL, Ramaekers FC, Bonne G, Yaou RB, Hutchison CJ. Nuclear lamins: laminopathies and their role in premature ageing. Physiol Rev 2006; 86:967-1008.

3. Chi YH, Chen ZJ, Jeang KT. The nuclear envelopathies and human diseases. J Biomed Sci 2009; 16:96.

4. Holt I, Ostlund C, Stewart CL, Man N, Worman HJ, Morris GE. Effect of pathogenic mis-sense mutations in lamin A on its interaction with emerin in vivo. J Cell Sci 2003; 116:3027-35.

5. Pegoraro G, Kubben N, Wickert U, Gohler H, Hoffmann K, Misteli T. Ageing-related chromatin defects through loss of the NURD complex. Nat Cell Biol 2009; 11:1261-7.

6. Dreger M, Bengtsson L, Schoneberg T, Otto H, Hucho F. Nuclear envelope proteomics: novel integral membrane proteins of the inner nuclear membrane. Proc Natl Acad Sci U S A 2001; 98:11943-8.

7. Holaska JM, Wilson KL. An emerin "proteome": purification of distinct emerincontaining complexes from HeLa cells suggests molecular basis for diverse roles including gene regulation, mRNA splicing, signaling, mechanosensing, and nuclear architecture. Biochemistry 2007; 46:8897-908.

8. Korfali N, Fairley EA, Swanson SK, Florens L, Schirmer EC. Use of sequential chemical extractions to purify nuclear membrane proteins for proteomics identification. Methods Mol Biol 2009; 528:201-25.

9. Lin F, Morrison JM, Wu W, Worman HJ. MAN1, an integral protein of the inner nuclear membrane, binds Smad2 and Smad3 and antagonizes transforming growth factor-beta signaling. Hum Mol Genet 2005; 14:437-45.

10. Schirmer EC, Florens L, Guan T, Yates JR, 3rd, Gerace L. Nuclear membrane proteins with potential disease links found by subtractive proteomics. Science 2003; 301:13802.

11. Wilkinson FL, Holaska JM, Zhang Z, Sharma A, Manilal S, Holt I, et al. Emerin interacts in vitro with the splicing-associated factor, YT521-B. Eur J Biochem 2003; 270:245966.

12. Zhong N, Radu G, Ju W, Brown WT. Novel progerin-interactive partner proteins hnRNP E1, EGF, Mel 18, and UBC9 interact with lamin A/C. Biochem Biophys Res Commun 2005; 338:855-61.

13. Van Berlo JH, Voncken JW, Kubben N, Broers JL, Duisters R, van Leeuwen RE, et al. Atype lamins are essential for TGF-beta1 induced PP2A to dephosphorylate transcription factors. Hum Mol Genet 2005; 14:2839-49.

14. Rybkin, II, Markham DW, Yan Z, Bassel-Duby R, Williams RS, Olson EN. Conditional expression of SV40 T-antigen in mouse cardiomyocytes facilitates an inducible switch from proliferation to differentiation. J Biol Chem 2003; 278:15927-34.

15. Sullivan T, Escalante-Alcalde D, Bhatt H, Anver M, Bhat N, Nagashima K, et al. Loss of A-type lamin expression compromises nuclear envelope integrity leading to muscular dystrophy. J Cell Biol 1999; 147:913-20.

16. Scaffidi $P$, Misteli T. Reversal of the cellular phenotype in the premature aging disease Hutchinson-Gilford progeria syndrome. Nature medicine 2005; 11:440-5.

17. Scaffidi P, Misteli T. Lamin A-dependent nuclear defects in human aging. Science (New York, NY 2006; 312:1059-63. 
18. IBA bioTAGnology. Expression and purification of proteins using Strep-tag and/or 6xHistidine-tag. A comprehensive Manual. 2005.

19. Sanchez C, Sanchez I, Demmers JA, Rodriguez P, Strouboulis J, Vidal M. Proteomics analysis of Ring1B/Rnf2 interactors identifies a novel complex with the $\mathrm{Fbxl10/Jhdm1B} \mathrm{histone} \mathrm{demethylase} \mathrm{and} \mathrm{the} \mathrm{Bcl6} \mathrm{interacting} \mathrm{corepressor.} \mathrm{Mol} \mathrm{Cell}$ Proteomics 2007; 6:820-34.

20. Wilm M, Shevchenko A, Houthaeve $T$, Breit $S$, Schweigerer $L$, Fotsis $T$, et al. Femtomole sequencing of proteins from polyacrylamide gels by nano-electrospray mass spectrometry. Nature 1996; 379:466-9.

21. Alibes A, Yankilevich P, Canada A, Diaz-Uriarte R. IDconverter and IDClight: conversion and annotation of gene and protein IDs. BMC Bioinformatics 2007; 8:9.

22. Dennis G, Jr., Sherman BT, Hosack DA, Yang J, Gao W, Lane HC, et al. DAVID: Database for Annotation, Visualization, and Integrated Discovery. Genome Biol 2003; 4:P3.

23. Huang da W, Sherman BT, Lempicki RA. Systematic and integrative analysis of large gene lists using DAVID bioinformatics resources. Nat Protoc 2009; 4:44-57.

24. Reimand J, Kull M, Peterson H, Hansen J, Vilo J. g:Profiler--a web-based toolset for functional profiling of gene lists from large-scale experiments. Nucleic Acids Res 2007; 35:W193-200.

25. Boehmer T, Jeudy S, Berke IC, Schwartz TU. Structural and functional studies of Nup107/Nup133 interaction and its implications for the architecture of the nuclear pore complex. Mol Cell 2008; 30:721-31.

26. Fontoura BM, Blobel G, Matunis MJ. A conserved biogenesis pathway for nucleoporins: proteolytic processing of a 186-kilodalton precursor generates Nup98 and the novel nucleoporin, Nup96. J Cell Biol 1999; 144:1097-112.

27. Frosst P, Guan T, Subauste C, Hahn K, Gerace L. Tpr is localized within the nuclear basket of the pore complex and has a role in nuclear protein export. J Cell Biol 2002; 156:617-30.

28. Jones FS, Meech R, Edelman DB, Oakey RJ, Jones PL. Prx1 controls vascular smooth muscle cell proliferation and tenascin-C expression and is upregulated with Prx2 in pulmonary vascular disease. Circ Res 2001; 89:131-8.

29. Nakielny S, Shaikh S, Burke B, Dreyfuss G. Nup153 is an M9-containing mobile nucleoporin with a novel Ran-binding domain. EMBO J 1999; 18:1982-95.

30. Junttila MR, Saarinen S, Schmidt T, Kast J, Westermarck J. Single-step Strep-tag purification for the isolation and identification of protein complexes from mammalian cells. Proteomics 2005; 5:1199-203.

31. Witte CP, Noel LD, Gielbert J, Parker JE, Romeis T. Rapid one-step protein purification from plant material using the eight-amino acid Strepll epitope. Plant Mol Biol 2004; 55:135-47.

32. Young SG, Fong LG, Michaelis S. Prelamin A, Zmpste24, misshapen cell nuclei, and progeria--new evidence suggesting that protein farnesylation could be important for disease pathogenesis. J Lipid Res 2005; 46:2531-58.

33. Gao J, Liao J, Yang GY. CAAX-box protein, prenylation process and carcinogenesis. Am J Transl Res 2009; 1:312-25.

34. Vaughan A, Alvarez-Reyes M, Bridger JM, Broers JL, Ramaekers FC, Wehnert M, et al. Both emerin and lamin $\mathrm{C}$ depend on lamin A for localization at the nuclear envelope. J Cell Sci 2001; 114:2577-90.

35. Lang C, Krohne G. Lamina-associated polypeptide 2beta (LAP2beta) is contained in a protein complex together with A- and B-type lamins. Eur J Cell Biol 2003; 82:143-53. 
36. Strelkov SV, Schumacher J, Burkhard P, Aebi U, Herrmann H. Crystal structure of the human lamin A coil 2B dimer: implications for the head-to-tail association of nuclear lamins. J Mol Biol 2004; 343:1067-80.

37. Grady RM, Starr DA, Ackerman GL, Sanes JR, Han M. Syne proteins anchor muscle nuclei at the neuromuscular junction. Proc Natl Acad Sci U S A 2005; 102:4359-64.

38. Haag J, Aigner T. Identification of calponin 3 as a novel Smad-binding modulator of BMP signaling expressed in cartilage. Exp Cell Res 2007; 313:3386-94.

39. Kimura E, Hidaka K, Kida Y, Morisaki H, Shirai M, Araki K, et al. Serine-arginine-rich nuclear protein Luc7l regulates myogenesis in mice. Gene 2004; 341:41-7.

40. Noon AT, Shibata A, Rief N, Lobrich M, Stewart GS, Jeggo PA, et al. 53BP1-dependent robust localized KAP-1 phosphorylation is essential for heterochromatic DNA doublestrand break repair. Nat Cell Biol 2010; 12:177-84.

41. Song $K$, Jung $Y$, Jung $D$, Lee I. Human Ku70 interacts with heterochromatin protein 1alpha. J Biol Chem 2001; 276:8321-7.

42. Swingler TE, Kevorkian L, Culley KL, IIlman SA, Young DA, Parker AE, et al. MMP28 gene expression is regulated by Sp1 transcription factor acetylation. Biochem J 2010; 427:391-400.

43. Zhang L, Kanda Y, Roberts DJ, Ecker JL, Losel R, Wehling M, et al. Expression of progesterone receptor membrane component 1 and its partner serpine 1 mRNA binding protein in uterine and placental tissues of the mouse and human. Mol Cell Endocrinol 2008; 287:81-9.

44. Joshi-Tope G, Gillespie M, Vastrik I, D'Eustachio P, Schmidt E, de Bono B, et al. Reactome: a knowledgebase of biological pathways. Nucleic Acids Res 2005; 33:D42832.

45. Holmberg A, Blomstergren A, Nord O, Lukacs M, Lundeberg J, Uhlen M. The biotinstreptavidin interaction can be reversibly broken using water at elevated temperatures. Electrophoresis 2005; 26:501-10.

46. Yoon E, Beom S, Cheong H, Kim S, Oak M, Cho D, et al. Differential regulation of phospholipase Cgamma subtypes through FcepsilonRI, high affinity IgE receptor. Biochem Biophys Res Commun 2004; 325:117-23.

47. Dechat T, Korbei B, Vaughan OA, Vlcek S, Hutchison CJ, Foisner R. Lamina-associated polypeptide 2a binds intranuclear A-type lamins. Journal of Cell Science 2000:347384.

48. Lobov IB, Tsutui K, Mitchell AR, Podgornaya OI. Specifcity of SAF-A and Lamin B Binding In Vitro Correlates With the Satellite DNA Bending State. Journal of Cellular Biochemistry 2001; 83:218-29.

49. Yang L, Guan T, Gerace L. Lamin-binding Fragment of LAP2 Inhibits Increase in Nuclear Volume during the Cell Cycle and Progression into S Phase. The Journal of Cell Biology 1997; 139:1077-87.

50. Ye Q, Worman HJ. Protein-Protein interactions between Human Nuclear Lamins Expressed in Yeast. Experimental Cell Research 1995; 219:292-98.

51. Mansharamani M, Wilson KL. Direct binding of nuclear membrane protein MAN1 to emerin in vitro and two modes of binding to barrier-to-autointegration factor. J Biol Chem 2005; 280:13863-70.

52. Razafsky D, Hodzic D. Bringing KASH under the SUN: the many faces of nucleocytoskeletal connections. J Cell Biol 2009; 186:461-72.

53. Ostlund C, Sullivan T, Stewart CL, Worman HJ. Dependence of diffusional mobility of integral inner nuclear membrane proteins on A-type lamins. Biochemistry 2006; 45:1374-82. 
54. Zhang SX, Garcia-Gras E, Wycuff DR, Marriot SJ, Kadeer N, Yu W, et al. Identification of direct serum-response factor gene targets during Me2SO-induced P19 cardiac cell differentiation. J Biol Chem 2005; 280:19115-26.

55. Sakaki M, Koike H, Takahashi N, Sasagawa N, Tomioka S, Arahata K, et al. Interaction between emerin and nuclear lamins. J Biochem 2001; 129:321-7.

56. Goldberg M, Lu H, Stuurman N, Ashery-Padan R, Weiss AM, Yu J, et al. Interactions among Drosophila nuclear envelope proteins lamin, otefin, and YA. Mol Cell Biol 1998; 18:4315-23.

57. Libotte T, Zaim H, Abraham S, Padmakumar VC, Schneider M, Lu W, et al. Lamin A/Cdependent localization of Nesprin-2, a giant scaffolder at the nuclear envelope. Mol Biol Cell 2005; 16:3411-24.

58. Dahl KN, Ribeiro AJ, Lammerding J. Nuclear shape, mechanics, and mechanotransduction. Circ Res 2008; 102:1307-18.

59. Krull S, Dorries J, Boysen B, Reidenbach S, Magnius L, Norder H, et al. Protein Tpr is required for establishing nuclear pore-associated zones of heterochromatin exclusion. EMBO J 2010.

60. Smythe C, Jenkins HE, Hutchison CJ. Incorporation of the nuclear pore basket protein nup153 into nuclear pore structures is dependent upon lamina assembly: evidence from cell-free extracts of Xenopus eggs. EMBO J 2000; 19:3918-31.

61. Walther TC, Fornerod M, Pickersgill H, Goldberg M, Allen TD, Mattaj IW. The nucleoporin Nup153 is required for nuclear pore basket formation, nuclear pore complex anchoring and import of a subset of nuclear proteins. EMBO J 2001; 20:5703-14.

62. Busch A, Kiel T, Heupel WM, Wehnert M, Hubner S. Nuclear protein import is reduced in cells expressing nuclear envelopathy-causing lamin A mutants. Exp Cell Res 2009; 315:2373-85.

63. Goldman RD, Shumaker DK, Erdos MR, Eriksson M, Goldman AE, Gordon LB, et al. Accumulation of mutant lamin $A$ causes progressive changes in nuclear architecture in Hutchinson-Gilford progeria syndrome. Proc Natl Acad Sci U S A 2004; 101:8963-8.

64. ten Berge D, Brouwer A, Korving J, Martin JF, Meijlink F. Prx1 and Prx2 in skeletogenesis: roles in the craniofacial region, inner ear and limbs. Development 1998; 125:3831-42.

65. Bergo MO, Gavino B, Ross J, Schmidt WK, Hong C, Kendall LV, et al. Zmpste24 deficiency in mice causes spontaneous bone fractures, muscle weakness, and a prelamin A processing defect. Proc Natl Acad Sci U S A 2002; 99:13049-54.

66. Csoka AB, English SB, Simkevich CP, Ginzinger DG, Butte AJ, Schatten GP, et al. Genome-scale expression profiling of Hutchinson-Gilford progeria syndrome reveals widespread transcriptional misregulation leading to mesodermal/mesenchymal defects and accelerated atherosclerosis. Aging Cell 2004; 3:235-43.

67. Varga R, Eriksson M, Erdos MR, Olive M, Harten I, Kolodgie F, et al. Progressive vascular smooth muscle cell defects in a mouse model of Hutchinson-Gilford progeria syndrome. Proc Natl Acad Sci U S A 2006; 103:3250-5.

68. Musich PR, Zou Z. Genomic instability and DNA damage responses in progeria arising from defective maturation of prelamin A. Aging (Albany NY) 2009; 1:28-37.

69. Nitta RT, Jameson SA, Kudlow BA, Conlan LA, Kennedy BK. Stabilization of the retinoblastoma protein by A-type nuclear lamins is required for INK4A-mediated cell cycle arrest. Mol Cell Biol 2006; 26:5360-72.

70. Maraldi NM, Lattanzi G, Capanni C, Columbaro M, Mattioli E, Sabatelli P, et al. Laminopathies: a chromatin affair. Adv Enzyme Regul 2006; 46:33-49. 
72 | Chapter 3

71. Huang da W, Sherman BT, Tan Q, Kir J, Liu D, Bryant D, et al. DAVID Bioinformatics Resources: expanded annotation database and novel algorithms to better extract biology from large gene lists. Nucleic Acids Res 2007; 35:W169-75.

72. D'Angelo MA, Hetzer MW. Structure, dynamics and function of nuclear pore complexes. Trends Cell Biol 2008; 18:456-66. 


\section{Supplemental Figures and Tables}

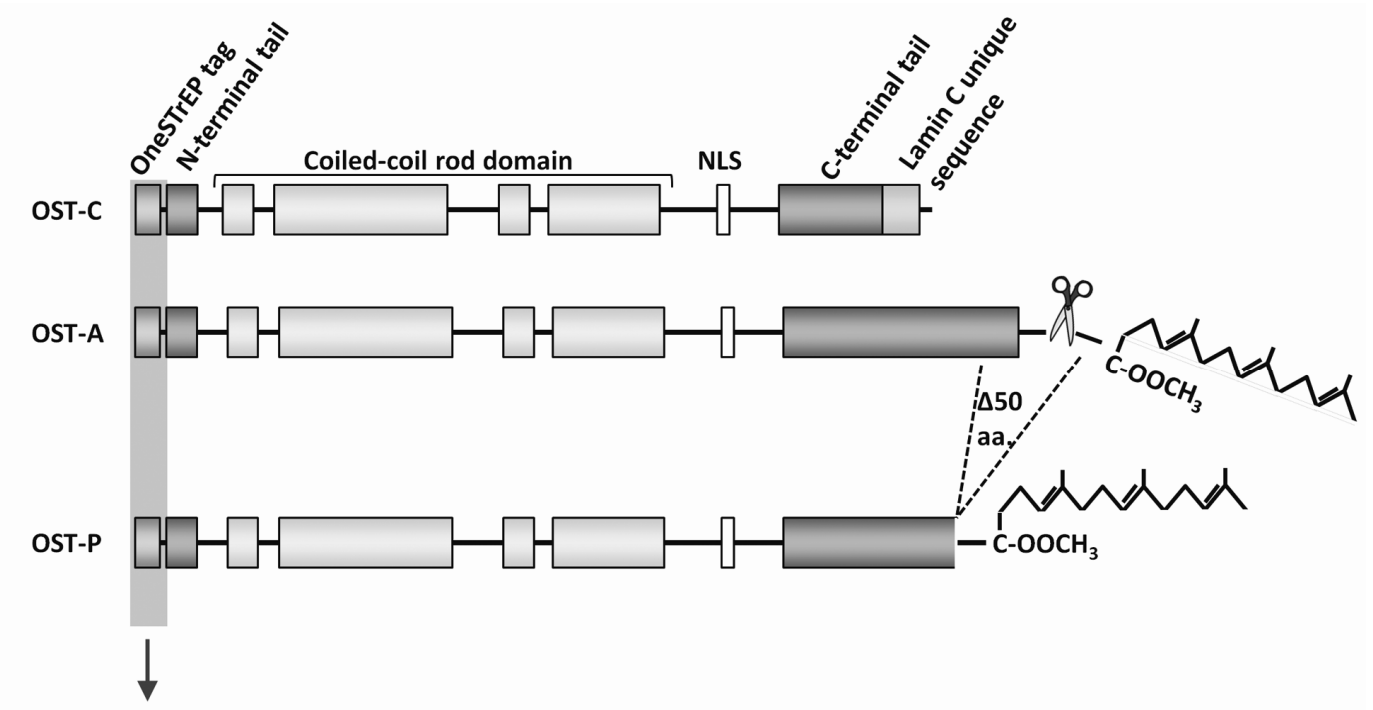

SAWSHPQFEKGGGSGGGSGGSAWSHPQFEK- Lamin Protein

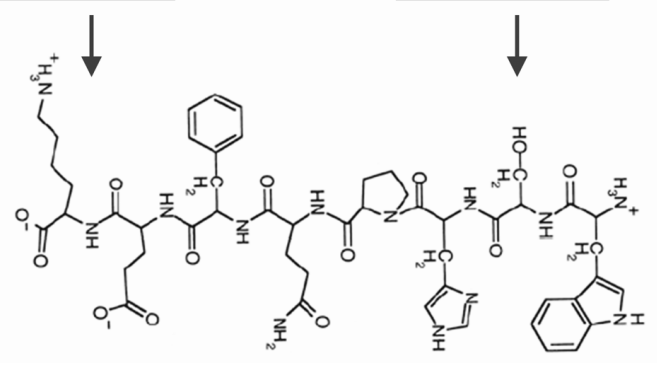

Figure S1. Schematic overview of OneSTrEP-tagged lamin constructs. Lamin A, C and progerin were tagged at their N-terminus by a OneSTrEP-tag, consisting of two Strep-tag II epitopes (underlined), which interact with high affinity to Streptactin matrix, separated by a serine and alanine linker region. The chemical structure of a Strep-tag II epitope is furher depicted. Mature OneSTrEP-tagged lamin A (OST-A) is released after farnesylation of the Cterminal Caax-motif, cleavage of the -aax amino acids and subsequent carboxymethyation of the C-terminus, followed by an upstream cleavage by Zmpste24 in mice cells (indicated by scissor; Face-1 for human cell lines). Due to a loss of 50 amino acids (indicated as $\Delta 50$ aa.), including the Zmpste24 restriction site, OneSTrEP-tagged progerin (OST-P) is only processed by farnesylation of the C-terminal Caax-motif, subsequent cleavage of the -aax amino acids and carboxymethyation of the C-terminus, and cannot be further cleaved by Zmpste24. OneSTrEP-tagged lamin C (OST-C), contains a Lamin C-specific terminus, as well as the A-type lamins common coiled-coil rod domain and nuclear localization signal (NLS). 


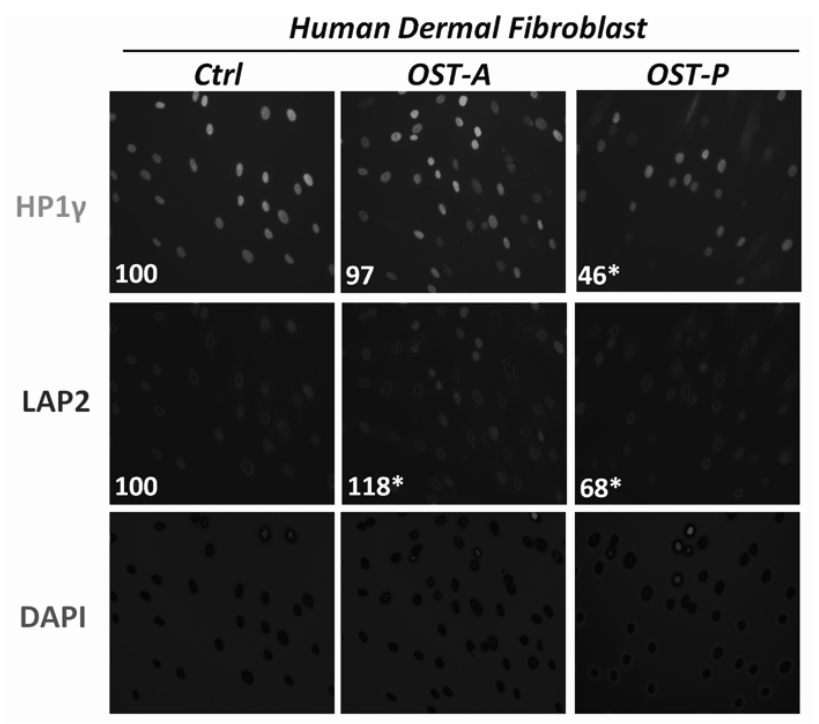

Figure S2. Progerin dependent loss of HP1Y and LAP2 protein. Fluorescent detection of HP1Y, LAP2 and DNA (DAPI) in human dermal fibroblasts (HDF) expressing an empty vector, OST-A or OST-P at endogenous lamin A levels. The nuclear intensity of total LAP2 (all isoforms included) and HP1 $\gamma$ was determined as described in the Material and Methods, and average expression values are depicted in numbers. Distribution curves were tested to be different or comparable using a Kolmogorov-Smirnov statistical test. An asterisk indicates a significant $(p<0.01)$ difference between HDFs expressing OST-A or OST-P in comparison to empty vector (Ctrl) infected HDFs. The following antibodies were used: $\alpha-\mathrm{HP} 1 \gamma$, mab3450, Chemicon, Temecula, USA ; $\alpha$-LAP2, kind gift from K. Wilson, raised against residues 1-187 of human LAP2, and suitable for detection of all LAP2 isoforms).

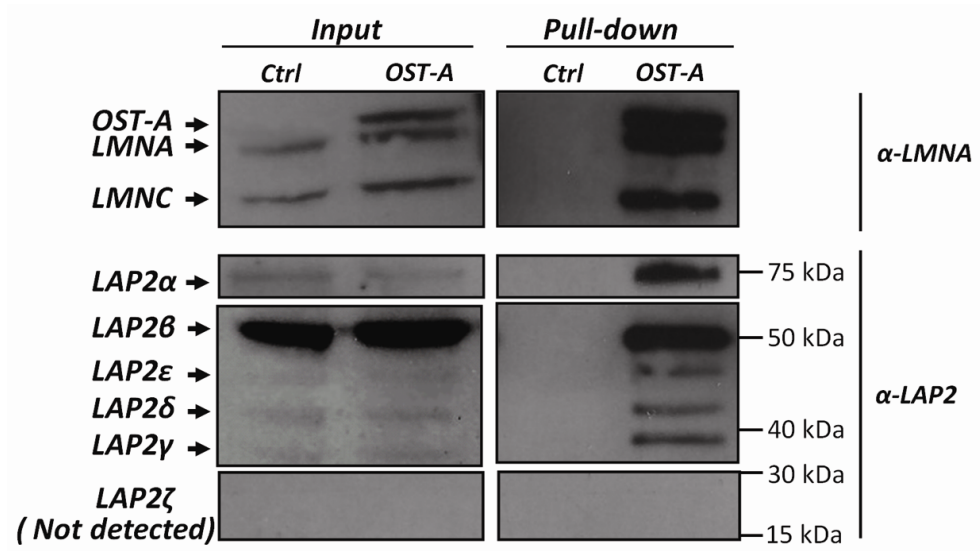

Figure S3. Interactions between Lamin A and LAP2 isoforms. Western blot of a OneSTrEP pull-down performed on U2OS cells infected with OST-A and control virus. Input and pulldown fractions were immunoblotted with $\alpha$-LaminA/C (Sc6215, Goat, Santa Cruz; detects endogenous Lamin A and C as well as OST-A) and $\alpha$-LAP2 (Y-20, Sc19783, Goat, Santa Cruz; suitable for LAP $2 \alpha, \beta, \gamma, \delta, \varepsilon$ and $\zeta$ detection). With the exception of LAP $2 \zeta$, which could not be detected, all other isoforms were immuoblotted at their respective molecular weights. 


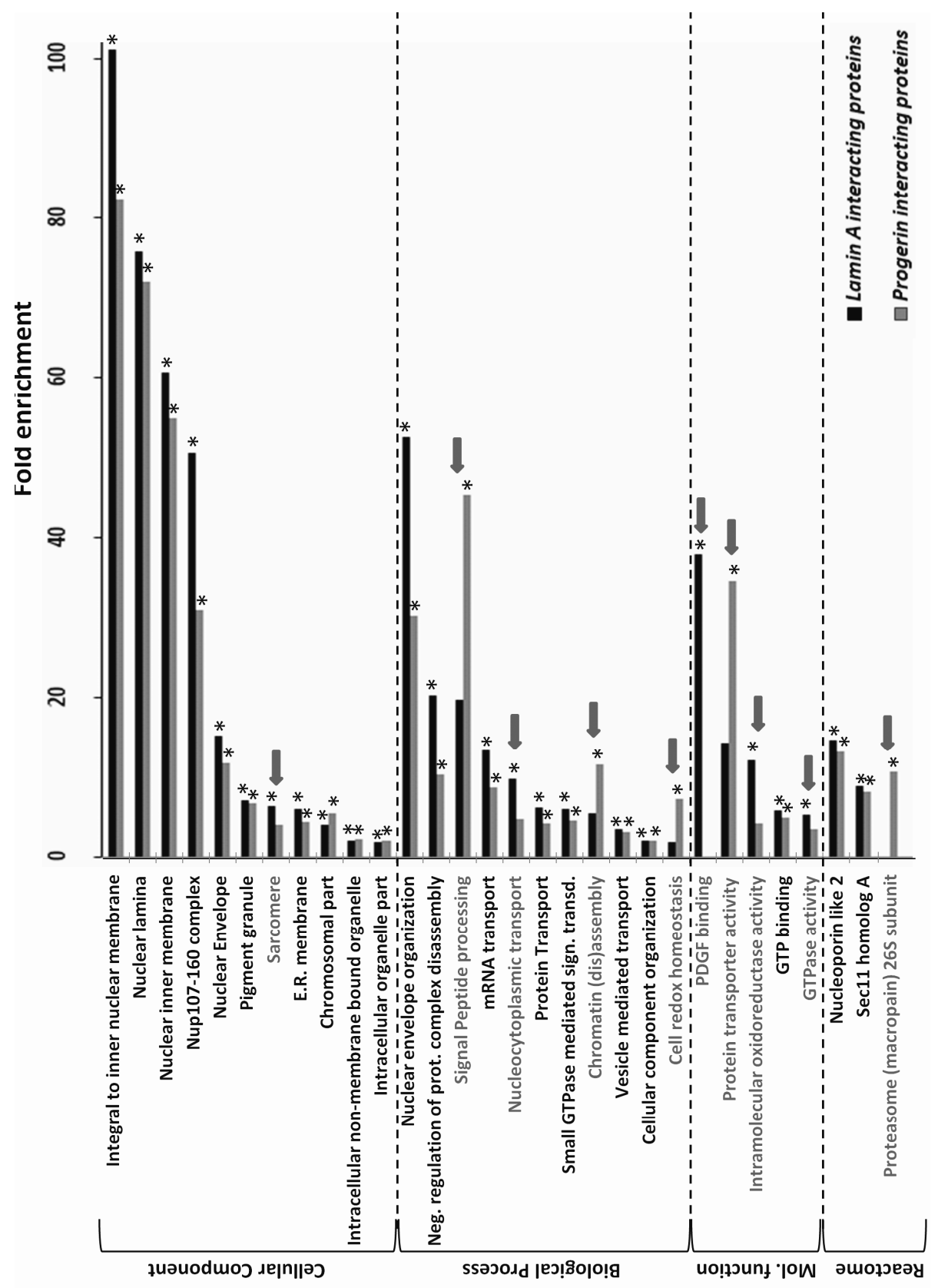

Figure S4. GO analysis of lamin A and progerin interacting proteins identified in MEFs. Identified lamin A and progerin interacting proteins were analyzed separately for the GO categories "cellular components" (CC), "biological process" (BP), "molecular function" (MF), as well as the reactome (R) database. Significant categories were determined by standard statistical algorithms incorporated into the DAVID database (see Material \& Methods) and are indicated by asterisks $\left(p<1 \times 10^{-3}\right.$; random proteins used as background). Categories enriched for by either OST-A or OST-P are indicated by arrows. 
Table S1. Mass spectrometry data for identified lamin A interacting proteins in NkITAg cells.

\begin{tabular}{|c|c|c|c|c|c|c|}
\hline Gl code & $\begin{array}{l}\text { Entrez } \\
\text { M.M. }\end{array}$ & Name & 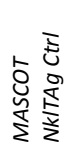 & 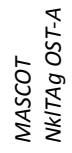 & 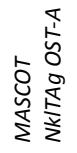 & $\begin{array}{c}\text { Previously } \\
\text { identified } \\
\text { in NE? }\end{array}$ \\
\hline 74219697 & 16905 & Lmna & 75 & 3534 & 3230 & $Y_{e s}^{6,10}$ \\
\hline 52869 & 16906 & Lmnb1 & & 2272 & 2122 & $Y e s^{6,10}$ \\
\hline 27503473 & 16907 & Lmnb2 & & 1230 & 1296 & $Y_{e s}^{6,10}$ \\
\hline 74202572 & 21917 & Tmpo & & 1154 & 1152 & $Y_{e s}^{6,10}$ \\
\hline 9845253 & 56258 & Hnrph2 & & 379 & 370 & $\mathrm{Yes}^{10}$ \\
\hline 9790069 & 53817 & Bat1a & & 350 & & $Y_{e s}{ }^{10}$ \\
\hline 28192672 & 98386 & Lbr & & 342 & 378 & $Y_{e s}^{6,10}$ \\
\hline 103484305 & 208263 & Tor1aip1 & & 327 & 118 & Yes $^{10}$ \\
\hline 114612484 & 628648 & HP1g & & 302 & 185 & $Y_{e s}{ }^{10}$ \\
\hline 50953786 & 223697 & Unc84b & & 302 & 166 & $Y_{e s}{ }^{10}$ \\
\hline 12852531 & 77053 & Unc84a & & 241 & 104 & $\operatorname{Yes}^{6,10}$ \\
\hline 20379977 & 232989 & Hnrpul1 & 63 & 234 & & $Y_{e s}{ }^{10}$ \\
\hline 74180977 & 17886 & Myh9 & & 220 & 39 & $Y_{e s}^{6,10}$ \\
\hline 3065929 & 22628 & Ywhag & & 198 & 204 & $Y_{e s}{ }^{10}$ \\
\hline 12832758 & 100046125 & RAB5A & & 179 & & Yes $^{10}$ \\
\hline 6679641 & 13726 & Emd & & 174 & 211 & $Y_{e s}^{6,10}$ \\
\hline 554262 & 26900 & Ddx3y & & 172 & 119 & $\operatorname{Yes}^{10}$ \\
\hline 21704124 & 433739 & RBM10 & & 168 & & Yes $^{10}$ \\
\hline 7106546 & 383901 & Mme1 & & 163 & & $Y_{e s}{ }^{10}$ \\
\hline 1389682 & 16480 & Jup & & 147 & & $Y_{e s}{ }^{10}$ \\
\hline 77812697 & 22138 & Ttn & & 136 & & $Y_{e s}{ }^{10}$ \\
\hline 6919955 & 24010 & $\mathrm{Ik}$ & & 136 & 68 & $Y_{e s}{ }^{10}$ \\
\hline 4507785 & 100043681 & UBC9 & & 132 & & Yes $^{10}$ \\
\hline 21312784 & 100047806 & PPIL1 & 32 & 127 & & $Y_{e s}{ }^{10}$ \\
\hline 267150 & 21973 & Top2a & & 123 & & $Y^{10}{ }^{10}$ \\
\hline 10946940 & 59021 & Rab2 & & 117 & 172 & Yes $^{10}$ \\
\hline 6755863 & 22027 & Hsp90b1 & & 113 & & $Y_{e s}{ }^{10}$ \\
\hline 145864461 & 380664 & Lemd3 & & 113 & 89 & $Y_{e s}^{6,10}$ \\
\hline 26325884 & 624311 & IPO2 & & 111 & & $Y_{e s}{ }^{10}$ \\
\hline 145699091 & 319565 & Syne2 & & 95 & 106 & $Y_{e s}{ }^{10}$ \\
\hline 148670589 & 19345 & Rab5c & & & 313 & Yes $^{10}$ \\
\hline 4501887 & 667260 & actG & & 677 & & No \\
\hline 34147513 & 19349 & Rab7 & 93 & 387 & 297 & No \\
\hline 13430890 & 50709 & Hist1h1e & & 244 & 226 & No \\
\hline 5902786 & 100045085 & ANX3 & & 242 & 81 & No \\
\hline 9055370 & 54709 & Eif3i & & 232 & 44 & No \\
\hline 109735018 & 239673 & 4732456N10Rik & & 226 & & No \\
\hline 7710086 & 19325 & Rab10 & & 220 & 219 & No \\
\hline 51092301 & 406223 & EG406223 & & 196 & & No \\
\hline 12857003 & 66978 & Luc7l & & 192 & & No \\
\hline 6671694 & 12419 & Cbx5 & 47 & 179 & 129 & No \\
\hline 12229677 & 11867 & Arpc1b & & 178 & & No \\
\hline 30424655 & 270192 & Rab6b & & 178 & & No \\
\hline 21703344 & 20224 & Sar1a & & 172 & 179 & No \\
\hline 109081395 & 22003 & Tpm1 & & 162 & & No \\
\hline 9790159 & 56305 & Pitpnb & & 158 & & No \\
\hline 1621617 & 21849 & Trim28 & 75 & 153 & 298 & No \\
\hline 12845035 & 675981 & eif2a & & 148 & & No \\
\hline 6755448 & 20333 & $\operatorname{Sec} 22 b$ & & 143 & 62 & No \\
\hline
\end{tabular}




\begin{tabular}{ccccc|c}
\hline 12836024 & 66870 & Serbp1 & 129 & No \\
\hline 4063383 & 20901 & Strap & 33 & 118 & No \\
\hline 45544618 & 66902 & Mtap & 117 & No \\
\hline 27369836 & 217980 & Larp5 & 115 & No \\
\hline 21312564 & 218501 & cnn3 & 106 & No \\
\hline 149272300 & 100039086 & Gm15032 & 105 & No \\
\hline 6755204 & 19173 & Psmb5 & 105 & No \\
\hline 21595163 & 234407 & Glt25d1 & 104 & No \\
\hline 12846304 & 13885 & Esd & 104 & 100 & No \\
\hline 198637 & 14375 & Ku70 & 33 & 131 & No \\
\hline 124486993 & 433923 & EG433923 & & No
\end{tabular}

Mascot scores for identified lamin A interacting proteins in NkITAg cells are listed. Furthermore it is indicated whether identified lamin A interactors were identified in two previous NE proteome studies (see grey shaded column) which had identified proteins in an INM/lumen/ONM6 fraction or an INM/lamina/nucleoplasmic fraction10. Hashes indicate proteins GO-annotated as 'Extracellular region'.

Table S2. GO analysis for identified lamin A (NkITAg) and preferential lamin A/progerin (MEF) interactors.

\begin{tabular}{|c|c|c|c|c|c|c|c|}
\hline Protein Set & Database & Database Category & $\begin{array}{l}\text { Protein in } \\
\text { category/ } \\
\text { Total } \\
\text { annotated } \\
\text { Proteins }\end{array}$ & $\begin{array}{l}\text { Enrichment } \\
\text { Factor vs. } \\
\text { Random } \\
\text { Protein } \\
\text { Background }\end{array}$ & P-Value & $\begin{array}{l}\text { Enrichment } \\
\text { Factor vs. } \\
\text { NE Protein } \\
\text { Background }\end{array}$ & $P$-value \\
\hline NKLTAG all & GO-CC & GO:0005635 nuclear envelope & $12 \mid 52$ & 21.5 & $5.0 \mathrm{E}-12$ & 6.3 & $1.0 \mathrm{E}-07$ \\
\hline NKLTAG all & GO-CC & GO:0048770 pigment granule & $6 \mid 52$ & 19.3 & $1.3 \mathrm{E}-05$ & 6.2 & $2.2 \mathrm{E}-02$ \\
\hline NKLTAG all & GO-CC & GO:0015629 actin cytoskeleton & $6 \mid 52$ & 8.0 & 8.1E-04 & 2.8 & $2.8 \mathrm{E}-01$ \\
\hline NKLTAG all & GO-CC & GO:0005694 chromosome & $6 \mid 52$ & 4.3 & 9.7E-03 & 2.0 & $2.3 \mathrm{E}-01$ \\
\hline NKLTAG all & GO-BP & $\begin{array}{l}\text { GO:0006998 nuclear envelope } \\
\text { organization }\end{array}$ & $3 \mid 39$ & 121.5 & $2.5 \mathrm{E}-04$ & 27.5 & 2.1E-04 \\
\hline NKLTAG all & GO-BP & $\begin{array}{l}\text { GO:0031032 actomyosin structure } \\
\text { organization }\end{array}$ & $3 \mid 39$ & 43.8 & 2.0E-03 & 4.8 & $1.0 \mathrm{E}+00$ \\
\hline NKLTAG all & GO-BP & $\begin{array}{l}\text { GO:0006333 chromatin assembly or } \\
\text { disassembly }\end{array}$ & $3 \mid 39$ & 10.0 & 9.9E-03 & 1.2 & $1.0 \mathrm{E}+00$ \\
\hline NKLTAG all & GO-BP & GO:0015031 protein transport & $11 \mid 39$ & 6.2 & 5.6E-06 & 2.8 & $4.5 \mathrm{E}-02$ \\
\hline NKLTAG all & GO-MF & GO:0000166 nucleotide binding & $18 \mid 53$ & 2.4 & $5.5 \mathrm{E}-04$ & 1.5 & $1.2 \mathrm{E}-01$ \\
\hline MEF diff & GO-CC & GO:0005635 nuclear envelope & $4 \mid 30$ & 12.4 & $3.6 \mathrm{E}-03$ & & \\
\hline MEF diff & GO-BP & $\begin{array}{l}\text { GO:0034660 ncRNA metabolic } \\
\text { process }\end{array}$ & $4 \mid 29$ & 9.7 & 7.1E-03 & & \\
\hline MEF diff & GO-BP & GO:0015031 protein transport & $7 \mid 29$ & 5.3 & $1.4 \mathrm{E}-03$ & & \\
\hline MEF diff & GO-BP & GO:0016070 RNA metabolic process & $6 \mid 29$ & 4.4 & 8.7E-03 & & \\
\hline MEF diff & GO-MF & GO:0003924 GTPase activity & $4 \mid 29$ & 16.6 & $1.6 \mathrm{E}-03$ & & \\
\hline MEF diff & Reactome & 823753:RAN binding protein 2 & $4 \mid 12$ & 8.8 & $6.9 \mathrm{E}-03$ & & \\
\hline
\end{tabular}


Table S3. GO analysis for identified lamin A- and progerin-interacting proteins in MEFs.

\begin{tabular}{|c|c|c|c|c|c|c|c|c|c|c|}
\hline \multirow[b]{2}{*}{ Database } & \multirow[b]{2}{*}{ Database Category } & \multicolumn{3}{|c|}{$\begin{array}{c}\text { MEF OST-A } \\
\text { interacting proteins }\end{array}$} & \multicolumn{3}{|c|}{$\begin{array}{c}\text { MEF OST-P } \\
\text { interacting proteins }\end{array}$} & \multicolumn{3}{|c|}{$\begin{array}{l}\text { Previously identified } \\
\text { NE proteins }\end{array}$} \\
\hline & & 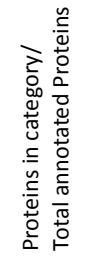 & 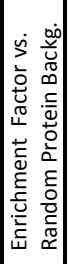 & $\frac{\stackrel{0}{\frac{2}{n}}}{\frac{1}{1}}$ & 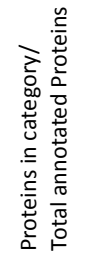 & 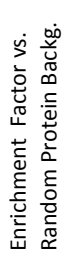 & $\frac{\stackrel{0}{\frac{2}{n}}}{\frac{1}{2}}$ & 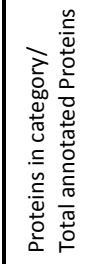 & 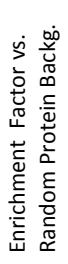 & $\frac{\frac{n}{2}}{\frac{2}{n}}$ \\
\hline GO-CC & $\begin{array}{l}\text { GO:0005639 integral to nuclear inner } \\
\text { membrane }\end{array}$ & $3 \mid 141$ & 101.1 & $2.9 \mathrm{E}-04$ & $3 \mid 173$ & 82.4 & $4.3 \mathrm{E}-04$ & $3 \mid 959$ & 14.9 & $1.3 \mathrm{E}-02$ \\
\hline GO-CC & GO:0005652 nuclear lamina & $6 \mid 141$ & 75.8 & 4.7E-09 & $7 \mid 173$ & 72.1 & $7.8 \mathrm{E}-11$ & $6 \mid 959$ & 11.1 & $6.4 \mathrm{E}-05$ \\
\hline GO-CC & GO:0005637 nuclear inner membrane & $9 \mid 141$ & 60.6 & $4.3 \mathrm{E}-13$ & $10 \mid 173$ & 54.9 & $2.1 \mathrm{E}-14$ & $9 \mid 959$ & 8.9 & $1.7 \mathrm{E}-06$ \\
\hline GO-CC & GO:0031080 Nup107-160 complex & $4 \mid 141$ & 50.5 & $5.0 \mathrm{E}-05$ & $3 \mid 173$ & 30.9 & $3.9 \mathrm{E}-03$ & $7 \mid 959$ & 13.0 & $2.3 \mathrm{E}-06$ \\
\hline GO-CC & GO:0005635 nuclear envelope & $23 \mid 141$ & 15.2 & $8.5 \mathrm{E}-20$ & $22 \mid 173$ & 11.8 & $1.3 \mathrm{E}-16$ & $63 \mid 959$ & 6.1 & $3.8 \mathrm{E}-33$ \\
\hline GO-CC & GO:0048770 pigment granule & $6 \mid 141$ & 7.1 & $1.5 \mathrm{E}-03$ & $7 \mid 173$ & 6.8 & $5.6 \mathrm{E}-04$ & $18 \mid 959$ & 3.1 & $4.2 \mathrm{E}-05$ \\
\hline GO-CC & GO:0030017 sarcomere & $5 \mid 141$ & 6.3 & $7.9 \mathrm{E}-03$ & $4 \mid 173$ & 4.1 & $7.2 \mathrm{E}-02$ & $16 \mid 959$ & 3.0 & $2.5 \mathrm{E}-04$ \\
\hline GO-CC & $\begin{array}{l}\text { GO:0005789 endoplasmic reticulum } \\
\text { membrane }\end{array}$ & $9 \mid 141$ & 6.0 & $1.3 \mathrm{E}-04$ & $8 \mid 173$ & 4.3 & $2.5 \mathrm{E}-03$ & $18 \mid 959$ & 1.8 & $2.7 \mathrm{E}-02$ \\
\hline GO-CC & GO:0044427 chromosomal part & $13 \mid 141$ & 4.1 & \begin{tabular}{|l|}
$7.0 \mathrm{E}-05$ \\
\end{tabular} & $21 \mid 173$ & 5.4 & $1.6 \mathrm{E}-09$ & $58 \mid 959$ & 2.7 & 7.0E-12 \\
\hline GO-CC & $\begin{array}{l}\text { GO:0043232 intracellular non-membrane- } \\
\text { bounded organelle }\end{array}$ & $40 \mid 141$ & 2.1 & $5.0 \mathrm{E}-06$ & $53 \mid 173$ & 2.3 & $7.2 \mathrm{E}-09$ & $279 \mid 959$ & 2.2 & $1.3 \mathrm{E}-39$ \\
\hline GO-CC & GO:0044446 intracellular organelle part & $60 \mid 141$ & 1.9 & $1.3 \mathrm{E}-07$ & $78 \mid 173$ & 2.0 & $5.4 \mathrm{E}-11$ & $495 \mid 959$ & 2.3 & $1.4 \mathrm{E}-93$ \\
\hline GO-BP & $\begin{array}{l}\text { GO:0006998 nuclear envelope } \\
\text { organization }\end{array}$ & $4 \mid 120$ & 52.7 & 4.6E-05 & $3 \mid 157$ & 30.2 & $4.1 \mathrm{E}-03$ & $6 \mid 876$ & 10.8 & 8.9E-05 \\
\hline GO-BP & $\begin{array}{l}\text { GO:0043242 negative regulation of } \\
\text { protein complex disassembly }\end{array}$ & $6 \mid 120$ & 20.3 & $1.0 \mathrm{E}-05$ & $4 \mid 157$ & 10.4 & $6.6 \mathrm{E}-03$ & $7 \mid 876$ & 3.3 & $9.1 \mathrm{E}-03$ \\
\hline GO-BP & GO:0006465 signal peptide processing & $1 \mid 120$ & 19.7 & $1.0 \mathrm{E}+00$ & $3 \mid 157$ & 45.3 & $1.7 \mathrm{E}-03$ & $3 \mid 876$ & 8.1 & $4.8 \mathrm{E}-02$ \\
\hline GO-BP & GO:0051028 mRNA transport & $7 \mid 120$ & 13.4 & $1.3 \mathrm{E}-05$ & $6 \mid 157$ & 8.8 & $5.8 \mathrm{E}-04$ & $43 \mid 876$ & 11.3 & $1.5 \mathrm{E}-36$ \\
\hline GO-BP & GO:0006913 nucleocytoplasmic transport & $8 \mid 120$ & 9.9 & $1.6 \mathrm{E}-05$ & $5 \mid 157$ & 4.7 & $2.1 \mathrm{E}-02$ & $31 \mid 876$ & 5.2 & 4.0E-14 \\
\hline GO-BP & GO:0015031 protein transport & $34 \mid 120$ & 6.2 & $1.6 \mathrm{E}-17$ & $30 \mid 157$ & 4.2 & $9.0 \mathrm{E}-11$ & $87 \mid 876$ & 2.2 & $9.1 \mathrm{E}-12$ \\
\hline GO-BP & $\begin{array}{l}\text { GO:0007264 small GTPase mediated signal } \\
\text { transduction }\end{array}$ & $13 \mid 120$ & 6.0 & $1.7 \mathrm{E}-06$ & $13 \mid 157$ & 4.6 & $2.7 \mathrm{E}-05$ & $13 \mid 876$ & 0.8 & $\underline{8.8 \mathrm{E}-01}$ \\
\hline GO-BP & $\begin{array}{l}\text { GO:0006333 chromatin assembly or } \\
\text { disassembly }\end{array}$ & $5 \mid 120$ & 5.4 & $\underline{1.3 \mathrm{E}-02}$ & $14 \mid 157$ & 11.6 & $1.9 \mathrm{E}-10$ & $27 \mid 876$ & 4.0 & $1.5 \mathrm{E}-09$ \\
\hline GO-BP & GO:0016192 vesicle-mediated transport & $14 \mid 120$ & 3.6 & $1.4 \mathrm{E}-04$ & $16 \mid 157$ & 3.1 & $1.9 \mathrm{E}-04$ & $31 \mid 876$ & 1.1 & 4.3E-01 \\
\hline GO-BP & $\begin{array}{l}\text { GO:0016043 cellular component } \\
\text { organization }\end{array}$ & $33 \mid 120$ & 2.1 & 5.7E-05 & $43 \mid 157$ & 2.1 & $4.1 \mathrm{E}-06$ & $192 \mid 876$ & 1.6 & $5.2 \mathrm{E}-13$ \\
\hline GO-BP & GO:0045454 cell redox homeostasis & $1 \mid 120$ & 1.9 & $1.0 \mathrm{E}+00$ & $5 \mid 157$ & 7.3 & 4.7E-03 & $0 \mid 876$ & 0.0 & \\
\hline GO-MF & $\begin{array}{l}\text { GO:0048407 platelet-derived growth } \\
\text { factor binding }\end{array}$ & $3 \mid 135$ & 38.0 & $2.6 \mathrm{E}-03$ & $0 \mid 167$ & 0.0 & & $3 \mid 926$ & 5.5 & $\underline{9.8 \mathrm{E}-02}$ \\
\hline GO-MF & $\begin{array}{l}\text { GO:0016864 intramolecular oxido- } \\
\text { reductase activity, transposing S-S bonds }\end{array}$ & $1 \mid 135$ & 14.3 & $\underline{1.0 \mathrm{E}+00}$ & $3 \mid 167$ & 34.6 & $3.1 \mathrm{E}-03$ & 이926 & 0.0 & \\
\hline GO-MF & GO:0008565 protein transporter activity & $7 \mid 135$ & 12.1 & $2.3 \mathrm{E}-05$ & $3 \mid 167$ & 4.2 & $1.6 \mathrm{E}-01$ & $14 \mid 926$ & 3.5 & $1.3 \mathrm{E}-04$ \\
\hline GO-MF & GO:0005525 GTP binding & $18 \mid 135$ & 5.8 & $1.2 \mathrm{E}-08$ & $19 \mid 167$ & 5.0 & $5.2 \mathrm{E}-08$ & $32 \mid 926$ & 1.5 & $\underline{2.3 \mathrm{E}-02}$ \\
\hline GO-MF & GO:0003924 GTPase activity & $6 \mid 135$ & 5.3 & $5.2 \mathrm{E}-03$ & $5 \mid 167$ & 3.6 & $5.0 \mathrm{E}-02$ & $22 \mid 926$ & 2.9 & $2.4 \mathrm{E}-05$ \\
\hline Reactome & 810245:nucleoporin like 2 & $10 \mid 56$ & 14.8 & \begin{tabular}{|l|l}
$7.2 \mathrm{E}-09$ \\
\end{tabular} & $10 \mid 62$ & 13.3 & $1.9 \mathrm{E}-08$ & $28 \mid 240$ & 9.6 & $2.1 \mathrm{E}-24$ \\
\hline Reactome & 783524:SEC11 homolog A (S. cerevisiae) & $4 \mid 56$ & 9.0 & $8.7 \mathrm{E}-03$ & $4 \mid 62$ & 8.1 & $9.8 \mathrm{E}-03$ & $4 \mid 240$ & 2.1 & $\underline{2.9 \mathrm{E}-01}$ \\
\hline Reactome & $\begin{array}{l}\text { 799755: proteasome (prosome, macropain) } \\
26 \text { S subunit, ATPase, } 2\end{array}$ & $0 \mid 56$ & 0.0 & & $10 \mid 62$ & 10.7 & -07 & 이240 & 0.0 & \\
\hline
\end{tabular}

Identified lamin A and progerin interacting proteins in MEFs, as well as previously identified NE proteins were analyzed separately for the GO categories "cellular components" (GO-CC), "biological process" (GO-BP), "molecular function" (GO-MF), as well as the reactome (R) database." ${ }^{6,10}$ Categories are listed together with the amount of proteins represented in the category versus to the total amount of annotated proteins, the enrichment factor compared to random protein sets ${ }^{21-22}$ and significance level. Significance $p$-values $(p<1 \times 10-3$; statistic testing includes kappa statistic measurement and is further described elsewhere ${ }^{66}$ ) are indicated; non-significant values are underlined or blank in case no proteins were found within the respective GO-term. 


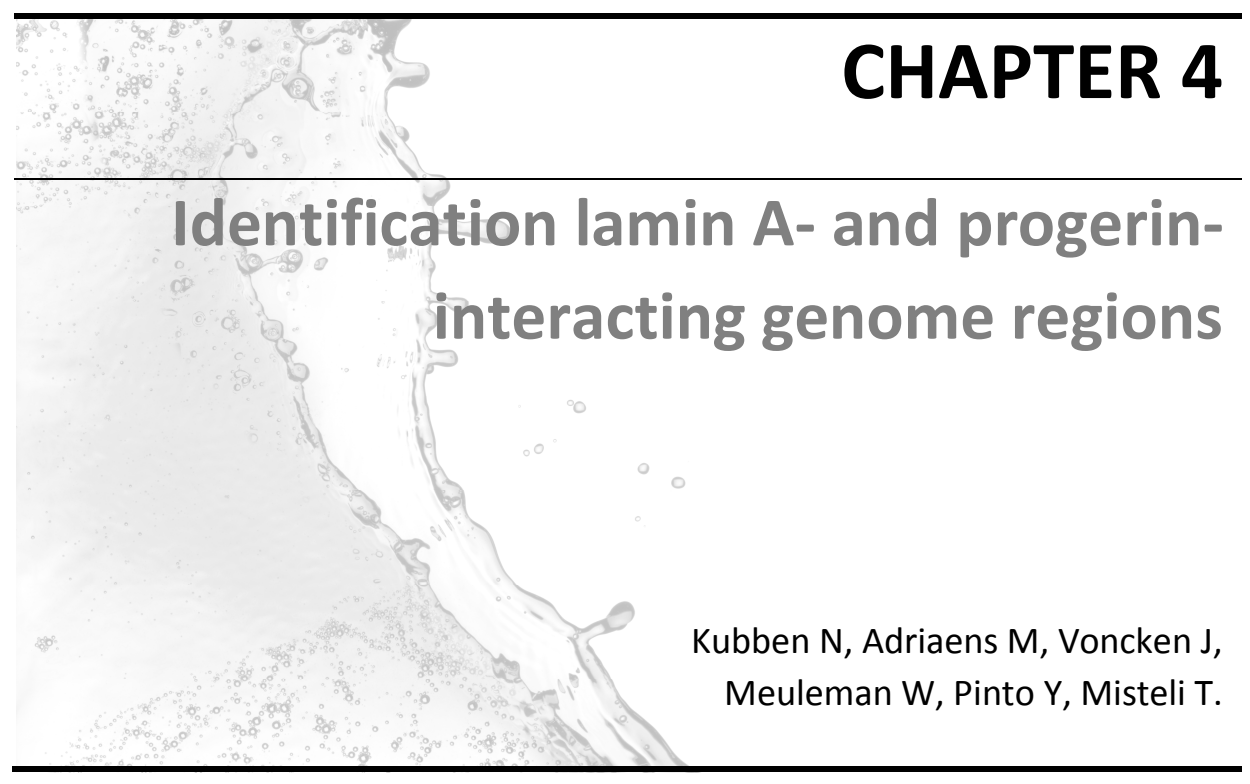




\begin{abstract}
Mutations in the A-type lamins $\mathrm{A}$ and $\mathrm{C}$, both major components of the nuclear lamina, cause phenotypically diverse diseases collectively referred to as laminopathies. These diseases often involve defects in chromatin organization, however, it is unclear whether aberrant chromatin-lamin interactions contributes to disease. Here we have used an unbiased approach to comparatively map genome-wide interactions of gene promoters with lamin A and progerin, the mutated lamin A isoform in the premature aging disorder Hutchinson-Gilford Progeria Syndrome (HGPS). We find that lamin A-associated genes are predominantly silent or expressed at low levels. We identify multiple classes of common or uniquely lamin A- or progerin-associated genes. Loss of lamin-association is not sufficient for gene activation and does not determine peripheral gene localization. Progerin induces global changes in chromatin organization and leads to relocation of peripherally localized genes, but not necessarily to their activation. These observations provide insight into the role of lamin A in chromatin organization and demonstrate that progerin affect specific chromatin-lamina interactions.
\end{abstract}




\section{Introduction}

The nuclear envelope (NE) defines the boundary between the nucleus and the cytoplasm and is composed of an outer and inner nuclear membrane (ONM, INM, respectively), interrupted by nuclear pore complexes (NPC) ${ }^{1}$. The NE is lined by a proteinaceous network made up of lamin proteins. The nuclear lamins are intermediate filament proteins and are categorized in Atype lamins (lamin $A, A \Delta 10, C$ and $C 2$ ), encoded by the LMNA gene, and $B-$ type lamins (lamin B1, B2 and B3), encoded by the LMNB1 and LMNB2 genes, respectively ${ }^{2}$.

Mutations in A-type lamins cause a group of phenotypically diverse diseases, collectively referred to as laminopathies ${ }^{3}$. They include several types of muscular dystrophies, lipodystrophies, cardiomyopathies, neurological disorders and premature ageing syndromes. A-type lamins are crucial in maintaining higher-order chromatin organization as loss of lamin $\mathrm{A} / \mathrm{C}$ results in reduction or loss of heterochromatin at discrete regions close to the INM ${ }^{4}$. Laminopathy-associated point mutations in lamin $A / C$ often deregulate chromatin structure and organization, most dramatically in the premature aging disease Hutchinson-Gilford Progeria Syndrome (HGPS). TheHGPS-associated mutant lamin A isoform progerin causes loss of heterochromatin and reduced mono- and tri-methylation of lysines 9 and 20 on histone $3^{5-6}$. Another progeria-related lamin A mutation, E145K, leads to alterations in pericentric chromatin, abnormal clustering of centromeres and mislocalization of telomeres ${ }^{7}$. Loss of heterochromatin and mislocalization of HP1 $\beta$ also occurs in mandibuloacral dysplasia (MAD) ${ }^{8-9}$.

Several lines of evidence suggest that A-type lamins interact directly with chromatin in vivo and that these interactions are affected by LMNA mutations. First, structurally related B-type lamins directly bind histones $\mathrm{H} 2 \mathrm{~A}$ and $\mathrm{H} 2 \mathrm{~B}$ in vitro ${ }^{10}$ and lamin $\mathrm{B}$ interacts with chromatin in vivo at discrete lamin-associated domains (LADs) which are characterized by low gene activity and demarcated by insulators ${ }^{11-13}$. Second, amino acids 396430 of the human lamin $A / C$ tail bind core histones in vitro ${ }^{14}$. Third, the immunoglobulin domain of lamin A/C covalently binds $30 \mathrm{bp}$ dimerized DNA fragments in vitro and the familial partial lipodystrophy (FPLD)-associated $\mathrm{R} 483 \mathrm{Q}$ and $-\mathrm{W}$ mutations lower this affinity ${ }^{15}$. Based on these findings it 
was hypothesized that direct interaction of A-type lamins with chromatin is important for chromatin organization and gene regulation ${ }^{16}$. However to date it is unknown which chromatin regions directly interact with A-type lamins in vivo, and how loss or mutation of lamin A/C affects chromatin organization and gene expression.

To probe the role of the lamina in genome organization and gene expression we have conducted an unbiased and genome-wide mapping of gene promoters that interact with lamin A and/or progerin. We find that lamin A preferentially binds silent or lowly expressed genes. This association facilitates, but does not determine, peripheral localization and loss of the interaction is not sufficient for gene activation. Progerin differentially interacts with many of the lamin A-associated genes and the basal expression level of those and common interactors correlates with their relative subnuclear position. These data demonstrate a direct and distinct effect of lamin A and progerin on chromatin interaction and organization at the lamina.

\section{Materials \& Methods}

\section{Cell lines and culture}

The lentiviral expression plasmid for $\mathrm{N}$-terminal One-STrEP tagged lamin A (OST-A) is described ${ }^{17}$. An N-terminal One-STrEP tagged progerin (OST-P) lentiviral expression plasmid was created by lamin A PCR amplification omitting amino acids $609-658$ using the PCDH MCSNard OST-A plasmid as a template and consequent ligation of the PCR product back into BamHI and EcoRI restriction sites. A lentiviral vector expressing shRNA directed against mouse lamin A/C (pSIHpuro-shRNA-mouseLMNA) was created by annealing 5'gatccGAGCTTGACTTCCAGAAGAACATttcaagagaATGTTCTTCTGGAAGTCAAG CTCtttttg $3^{\prime}$ and 5'aattcaaaaaGAGCTTGACTTCCAGAAGAACATtctcttgaaATGTTCTTCTGGAAGTC AAGCTCg 3' oligos and consequent ligation into BamHI and EcoRI restricted pSIH H1 plasmid (System Biosciences, Mountain View, USA). Lentivirus was produced by co-transfecting 293 FT cells (Invitrogen, Carlsbad, USA) with pSIHpuro-shRNA-mouseLMNA, pCDHblast MCSNard OST-A or OST-P in combination with PSPAX and PMD2.G vectors (a gift from D. Trono, 
Lausanne, Switzerland). The mouse cardiac myocyte cell line NkITAg ${ }^{18}$, as well as mouse embryonic fibroblasts of wild-type (WT) MEFs and LMNA knock-out (LMNA ${ }^{\mathrm{KO}-1}$ ) embryos ${ }^{4}$ were infected with various concentrations of lentivirus and 48 hours post infection selected with blasticidin. Infected cells with OST-A or OST-P protein expression levels comparable to endogenous lamin A were further cultured and grown in Dulbecco's modified Eagle's/F-12 media (for NkITAg cells) or Dulbecco's modified eagle medium (for MEFs) both supplemented with $2 \mathrm{mM}$ L-glutamine, $10 \mathrm{mM}$ nonessential amino acids (NEAA), $110 \mathrm{mg} / \mathrm{l}$ pyruvate, $10 \%$ fetal bovine serum and antibiotics in a humidified atmosphere at $37^{\circ} \mathrm{C}$ at $5 \% \mathrm{CO}_{2}$. NkITAg cells were plated on $12.5 \mu \mathrm{g} / \mathrm{ml}$ fibronectin and $0.1 \%$ gelatin coated plates.

\section{Western blot}

Western blots were performed essentially as described elsewhere ${ }^{17}$. Cells were lysed in SDS-PAGE Laemmli loading buffer and further denatured by heating for $5 \mathrm{~min}$ at $95^{\circ} \mathrm{C}$. Equal amounts of cell lysates were loaded and run on NuPage Novex Bis-Tris 4-20\% gradient gels (Invitrogen, Carlsbad, USA), blotted on immobilon-PVDF membrane (Millipore, Billerica, USA), blocked 1 hour in $5 \%$ bovine serum albumin /TBS-T (20 mM Tris-HCl pH 7.5, $150 \mathrm{mM}$ $\mathrm{NaCl}$ and $0.1 \%$ Tween 20). The blocked membrane was incubated at $4{ }^{\circ} \mathrm{C}$ overnight with primary antibodies ( $\alpha$-Lamin A/C, Sc6215, StCruz, Santa Cruz, USA; $\alpha$-Beta Actin, A-5441, Sigma, St Louis, USA) in blocking buffer diluted. After incubation with appropriate secondary HRP-labeled secondary antibodies, immunoluminescence was detected with an ECL Western Blotting detection system (Amersham, Roosendaal, Netherlands). The intensity of signals was quantified using ImageJ software.

\section{Immunofluorescence}

For immunofluorescence microscopy, cells were grown on $0.1 \%$ gelatin/PBS solution coated multi-wells glass slides (MP Biomedicals, Solon, USA), fixed for $15 \mathrm{~min}$ in $4 \%$ paraformaldehyde/PBS, permeabilized with $0.5 \%$ Triton X100 in PBS for 5 min, blocked for 30 minutes in block buffer (1\% BSA, 1\% sucrose in milliQ) and incubated for one hour at room temperature in appropriate primary antibodies ( $\alpha$-LMNA/C, Sc-7292, StCruz, Santa Cruz, USA) and for one hour with secondary antibodies labeled with the Alexa Fluor 488 or 568 chromophores (Invitrogen, Carlsbad, USA). Cells were 
mounted using Vectashield containing $10 \mathrm{~g} / \mathrm{ml}$ DAPI and observed on a Nikon E800 microscope.

\section{Chromatin Immunoprecipitation (ChIP)}

NkITAg and MEF cell lines were grown in $150 \mathrm{~mm}$ dishes, formaldehyde fixed and lysed in SDS lysis buffer (1\% SDS, $10 \mathrm{mM}$ EDTA, $50 \mathrm{mM}$ Tris $\mathrm{pH}$ 8.1). After sonication the SDS concentration was lowered 10 -fold by adding ChIP dilution buffer (0.01\% SDS, 1.1\% Triton X-100, 1.2 mM EDTA, $17 \mathrm{mM}$ Tris $\mathrm{pH}$ 8.1, $170 \mathrm{mM} \mathrm{NaCl}$ ). Both SDS lysis buffer and ChIP dilution buffer were supplemented with $2 \mathrm{mM}$ ortohovanadate and protease inhibitors (Boehringer MannHeim, Indianapolis, USA). Undissolved fractions were discarded after a 10 minute 16,000 g centrifugation step at 4 degrees. ChIP dilution buffer pre-washed and herring sperm DNA coated Strep-Tactin Matrix (IBA BioTagnology, Göttingen, Germany) was added and samples were end-over-end rotated overnight at 4 degrees. The next day pelleted Strep-Tactin matrix was washed consequently with low salt buffer $(0.1 \%$ SDS, 1\% Triton X-100, 2 mM EDTA, 20 mM Tris pH 8.1, $150 \mathrm{mM} \mathrm{NaCl),} \mathrm{high}$ salt buffer (0.1\% SDS, 1\% Triton X-100, 2 mM EDTA, $20 \mathrm{mM}$ Tris pH 8,1, 500 $\mathrm{mM} \mathrm{NaCl}$ ), LiCl buffer (0.25 M LiCl, 1\% Igepal-CA630, 1\% deoxycholic acid, 1 $\mathrm{mM}$ EDTA, $10 \mathrm{mM}$ Tris pH 8.1), OST stringent wash buffer (2M NaCl, 2\% Trx100, $500 \mathrm{mM} \mathrm{LiCl,} 0.1 \%$ SDS, 1\% Sodium Deoxycholate, $20 \mathrm{mM}$ Tris pH 8.1, $2 \mathrm{mM}$ EDTA) and TE buffer (10 mM Tris, 1mM EDTA pH 8.0). Precipitated protein-chromatin complexes were eluted at room temperature in fresh elution buffer (1\% SDS and $0.1 \mathrm{M} \mathrm{NaHCO}$ ). After de-cross-linking for 6 hours at $65^{\circ} \mathrm{C}$ in the presence of $200 \mathrm{mM} \mathrm{NaCl}$ and $30 \mu \mathrm{g} / \mathrm{ml}$ RNAase A, DNA was purified using a Qiagen PCR clean-up kit (Qiagen, Hilden, Germany) and amplified according to Whole Genome Amplification kit instructions (Sigma, St. Louis, USA) for further analysis on promoter arrays.

\section{ChIP-on-chip analysis}

Amplified DNA fragments were hybridized to NimbleGen MM8 385K Refseq promoter arrays, tiling a region of $2000 \mathrm{bp}$ downstream to $500 \mathrm{bp}$ upstream for transcription start sites of $\sim 19,000$ Refseq genes, excluding chromosomes 20 to 22. NkITAg OST-A targets were identified based the combined data from 4 independent experiments. Two arrays were hybridized with Cy-3 labeled input DNA and Cy-5 labeled ChIPed DNA from 
NkITAg cells. To correct for non-specific enrichment of DNA fragments ChIPs on cells transfected with an empty vector ( $\mathrm{Cy}-3$ labeled) served as a reference for ChIPs on OST-A expressing NkITAg cells (Cy-5 labeled) on two additional arrays. To identify differences in lamin $A$ and progerin promoter association, data of 4 independent experiments were combined. Lamin A and progerin targets were identified independently in WT and LMNA ${ }^{\mathrm{KO}-}-$ MEFs ${ }^{4}$ by hybridization of OST-A ChIPed DNA (Cy-5 labeled) against ChIPed DNA from empty vector infected cells (Cy-3 labeled), and hybridization of OST-P ChIPed DNA (Cy-5 labeled) against OST-A ChIPed DNA (Cy-3 labeled). Hybridization and data acquisition were performed in-house by NimbleGen according to standard procedures (Roche NimbleGen, Madison, USA).

To identify binding targets in the microarray data, we applied a betweenarray analysis approach in the statistical programming language $R$. This method compares all replicates and any negative controls directly at probe level, which is thereby expected to produce a statistically more robust set of potential targets compared to NimbleGen's standard within-array analysis method. Our approach is fully described elsewhere ${ }^{19}$. In short, a log-ratio between experimental and control samples are calculated separately for each array. Next, all probes are ordered according to genomic location and dichotomized using a threshold around twice the estimated standard deviation of the log-ratio. Probes with a log-ratio above this threshold are considered positive, those below negative. Next, for each array a sliding window of a variable number of base pairs is moved over all probes, calculating a detection $p$-value for the probes in each probe-window using a Yates corrected Chi-square test, to assess whether there are significantly more positive probes present than expected by chance. A promoter is significantly enriched when at least one probe-window shows significant enrichment in all experimental arrays for a particular condition and in none of any available arrays suitable as negative controls for that condition. To minimize false positives a detection $p$-value of 0.05 was used as the cut-off per array. OSTA- and OST-P associated gene promoters needed to be recognized as such independently in both WT and $\mathrm{LMNA}^{\mathrm{KO}-/-} \mathrm{MEFs}$, and are collectively referred to as MEF OST-A and OST-P ChIP targets.

Cluster analysis of identified lamin targets was essentially performed as described previously ${ }^{13}$. To test whether lamin targets were clustered, we 
defined clusters as two or more adjacent lamin-associated genes not interrupted by non-target genes. Next, unclustered (cluster size $=1$ gene) and clustered (cluster size>1 gene) lamin targets for all chromosomes were compared by Fisher's exact test to randomly expected occurrences of gene clusters, determined in 10,000 simulations.

Gene ontology (GO) analysis was performed with the Database for Annotation, Visualization, and Integrated Discovery (DAVID) using standard settings for functional annotation clustering, listing significant clusters by most significant GO Biological Processes class ${ }^{20-21}$. Average conservation plots were generated with Cis-regulatory Element Annotation System (CEAS) (http://ceas.cbi.pku.edu.cn/submit.htm) ${ }^{22}$, showing the level of conservation of the ChIP regions compared to the genomic background. MatInspector software and the Genomatix transcription factor motif database (www.genomatix.de) were used to identify enriched Transcription Factor Motifs (TFM) in target sites and target promoters ${ }^{23}$.

\section{Fluorescent In Situ Immunohybridization (FISH)}

To produce probes for DNA FISH, bacterial artificial chromosomes (BACs, BACPAC Resources Center, Oakland, USA) were labeled by nick translations with dUTP conjugated with biotin or dioxygenin (Roche, Madison, USA) using the mouse BAC clones (Acpp, RP24-383K20; Sp100, RP24-235A6; OLFR681, RP24-324M2; OLFR1471, RP24-346K11; Eif2b, RP24-285P5; Fanca, RP24-157M4; Eef1a2, RP24-163K20; Kcnip2, RP24-337I2; Scn5a, RP23103G4; Sdro, RP24-244F19; Сyp3a25, rp-23-332c3; Olfr804, rp24-167G2; Abpd, rp24-238l13; Defcr5, rp24-243l16; Daf2, RP24-154P21; Tcf21, RP2393MI0; Trim30, RP23-312K22; Abcb10, RP24-361K8) as described ${ }^{24-25}$. Probes consisted of 150-300 ng of digoxigenin- and/or biotin-labeled probe DNA, $3 \mu \mathrm{g}$ mouse COT1 DNA (Roche, Madison, USA) and $20 \mu \mathrm{g}$ tRNA (Sigma, St Louis, USA) resuspended in $7 \mu$ l of hybridization mix (10\% dextran sulfate, $50 \%$ formamide/2xSSC, and $1 \%$ Tween 20 ). Cells grown on glass slides were fixed in $4 \%$ PFA/PBS for $10 \mathrm{~min}$, permeabilized for $20 \mathrm{~min}$ in $0.5 \%$ saponin/0.5\% Triton X-100/PBS and incubated in $0.1 \mathrm{~N} \mathrm{HCl}$ for 15 min with PBS washes between each step. After a $2 x$ SSC wash, cells were equilibrated in $50 \%$ formamide/ $2 \times$ SSC. Nuclei and probes were denatured together at $85^{\circ} \mathrm{C}$ for $5 \mathrm{~min}$ and left to hybridize at $37^{\circ} \mathrm{C}$ overnight in a humidified 
chamber. The next day, slides were washed three times with $50 \%$ formamide/2xSSC at $45^{\circ} \mathrm{C}$ for 5 min each and thrice with $1 \times$ SSC at $60^{\circ} \mathrm{C}$ for 5 min each. Next, the cells were placed in $0.05 \%$ Tween-20/4xSSC at room temperature to cool and blocked for $20 \mathrm{~min}$ in 3\% BSA/0.05\% Tween20/4xSSC. For immunofluorescence of LMNB1 slides were incubated at room temperature for 45 minutes with 1:50 in block buffer diluted LMNB antibody (Sc-6217, Santa Cruz Biotechnology, Santa Cruz, USA). Next, detection antibodies (anti-dioxigenin-rhodamine; Roche, Madison, USA), fluorescein avidin DN (Vector Laboratories, Burlingame, USA) and Alexa Fluor 647 Donkey-anti-goat IgG (Invitrogen, Carlsbad, USA)) diluted 1:200 in blocking solution were incubated with cells for one hour at $37^{\circ} \mathrm{C}$. Slides were mounted in DAPI-containing Vectashield mounting medium after three 5min washes in $0.05 \%$ Tween-20/4xSSC at $42^{\circ} \mathrm{C}$.

\section{FISH image acquisition and analysis}

Cells were imaged with an IX70 microscope (Olympus) controlled by a Deltavision System (Applied Precision) with SoftWoRx 3.7.0 Release 13EL (Applied Precision) and fitted with a charge-coupled device camera (CoolSnap; Photometrics) using a 60x 1.4 oil objective lens (Olympus) and an auxiliary magnification of 1.5 using an optical step size of $0.2 \mu \mathrm{m}$. Typically, 25 focal sections were imaged covering the entire nucleus. Using SoftWoRx 3.7.0 Release 13EL (Applied Precision) FISH signals were detected and analyzed in the $\mathrm{z}$ section with the brightest signal intensity. The two brightest FISH signals were used for quantification and frequency of cells with minimally one FISH signal within $500 \mathrm{~nm}$ distance of (NkITAg experiments) or overlapping (MEF experiments) with LMNB staining were quantified. Minimally 150 nuclei were analyzed in duplicate per probe per cell line. Per probe quantification results were statistically tested between different cell lines using the chi-square test. To test whether the distribution of observed frequencies of cells with FISH signals at the NE for OST-Auniqe, common OST-A\&P and OST-Punique targets differ for different cell lines a Kruskal-Wallis One way analysis of variance by ranks was applied, using Dunn's method for pair-wise multiple comparisons. SigmaStat 3.1 software was used for statistical analysis. For all tests P-values $<0.05$ were considered significant. 


\section{RNA isolation and expression microarray}

RNA was isolated using the RNAeasy minikit (Qiagen, Hilden, Germany) from MEF, cardiac myocyte NkITAg cells and cardiac left ventricles of a gene trap LMNA null mouse model (LMNA ${ }^{\text {GT- }- \text { ) }}$ (Kubben et al., in preparation). For whole transcriptome analyses RNA from 5 days old $W T$ and $L M N A^{G T-/}$ mice $(\mathrm{N}=2$ each) and from WT MEFs infected with a OST-A, OST-P or empty vector lentivirus ( $\mathrm{N}=2$ each) were isolated and hybridized to Nugo Mouse Affymetrix expression arrays and Affymetrix Mouse Gene 1.0 ST arrays, respectively. Intensity values after hybridization were normalized to the median signal intensity of the array. For individual genes differences in expression levels were statistically tested by a one way ANOVA. Expression heatmaps were generated with DNA Arraystar 3.0 Software (DNAStar, Madison, USA). Expression profiles between different cell lines were statistically analyzed by first testing for normality of distribution by a Kolmogorov-Smirnov test. P-values tested were all significant $(p<0.05)$ hence expression values were not considered to be normally distributed. Consequently differences in expression values were tested with the nonparametric Mann-Whitney Rank Sum test between 2 groups, and the Kruskall-Wallis one-way analysis of variance for more than 2 groups. Pvalues below 0.05 were considered significant. Verification of microarray expression data by PCR cDNA was synthesized with the iScript ${ }^{\mathrm{TM}}$ CDNA synthesis kit (BioRad, Hercules, USA). SYBR Green real-time quantitative PCR analysis was performed in experimental duplos with primers for Beta-Actin (FW 5'TGgagCCTGtgtTCTATCCA3', Rev 5'TGgagCCTGtgtTCTATCCA3'), PCNA (FW 5'AGGGTTGGTAGTTGTCGCTGTAG3', Rev 5'GGTCCCCCGATTCACGAT3'), Sp100 (FW 5'AACGACGCAGAAAAAGAGGT3', Rev 5'GtGAATAACCGCCCTGTCTT3'), CdkI4 (FW 5'ACCAGGGCACCTCTAATCAC3', Rev 5'CTCTGGTGAAACTGGAAGCA3'), OLFR686 (FW 5'TGGATATCCCTTCCCTTCTG3', Rev 5'TGGAGCCTGTGTTCTATCCA3'), OLFR826 (FW 5'ACTCGGGCTTAGGAAGATGA3', Rev 5'GGATCATGGCATACACAAGC3'), OLFR1098 (FW 5'TGtCCTACATCGTTGGAGCTAT3', Rev 5'TGCAGAAATCCAGTGTGCTT3'), Defb28 (FW 5'TAGCTGTCCTTGTGGTCCTG3', Rev 5'CCAAGCCATAAAAGCAGGTT3'), Acpp (FW 5'CTGGGTCTTTTTCCCTGTGT3', Rev 5'ACTCGGGCTTAGGAAGATGA3') and HPRT (FW 5'GCGTCGTGATTAGCGATGATGAAC3', Rev 5' CCTCCCATCTCCTTCATGACATCT $3^{\prime}$ ). Differences in real-time PCR quantification were determined applying a student T-test with 0.05 significance level. 
A

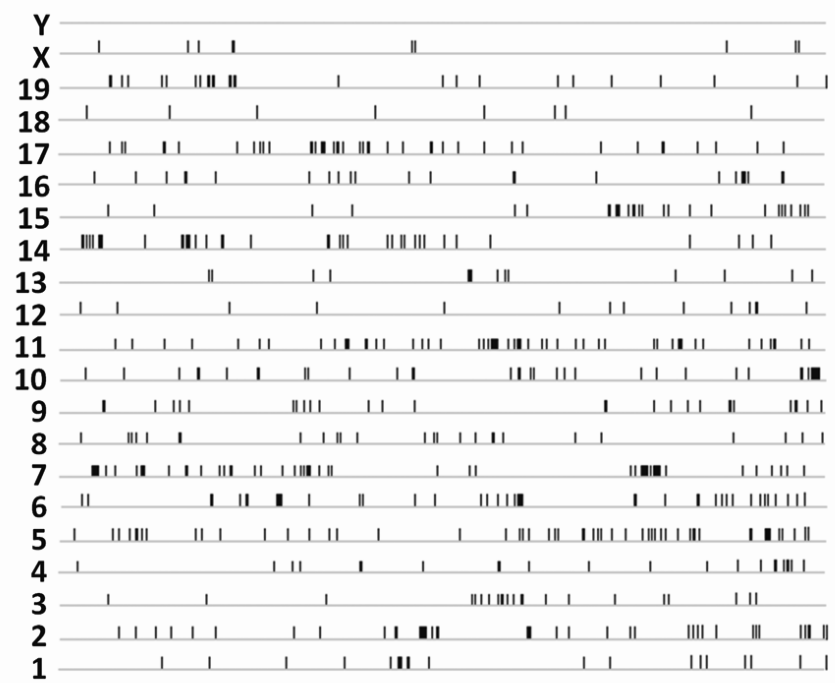

B

\begin{tabular}{lccc} 
Go Category & \multicolumn{3}{c}{$\begin{array}{c}\mathbf{N} / \text { All CC } \\
\text { targets for } \\
\mathbf{p} / \text { All }\end{array}$} \\
\hline \hline Gprotein coupled receptor signaling & $74 / 292$ & $30 / 292$ & P value \\
Locomotion & $11 / 292$ & $10 / 292$ & $1.0 \times 10^{-5}$ \\
Regulation of Blood Coagulation & $3 / 292$ & $2 / 292$ & $3.5 \times 10^{-2}$ \\
\hline \hline
\end{tabular}

Figure 1. Genomic localization and characteristics of lamin-associated genes. (A) Identified ChIP-chip targets of OST-LMNA in NkITAg myocytes are plotted according to their genomic location. Chromosomes are depicted in a relative scale, which corrects for size differences between individual chromosomes. (B) Summary of a gene ontology (GO) analysis on identified lamin A-targets. Per GO category the amount of lamin A-associated genes, out of a total of 292 targets, enriched for is indicated, as well as the minimal amount of genes needed to reach a significance level of 0.05 , based on statistical algorithms of the DAVID database described elsewhere 39.

\section{Results}

\section{Identification of lamin A-associated genes}

To map lamin A interacting chromatin regions genome-wide we stably expressed One-STrEP-tagged lamin A (OST-A) at endogenous levels in a murine myocyte NkITAg ${ }^{18}$ cell line ${ }^{26}$. The One-STrEP (OST) tag is a modified version of the biotin-related 8 amino acid Strepll epitope, which binds with high affinity $(\mathrm{Kd}=1 \mu \mathrm{M})$ to Strep-Tactin Matrix, an engineered streptavidin analogue ${ }^{27}$. OST-A has previously been characterized in detail and the OSTtag does not interfere with correct localization or function of lamin $A$ at the 
nuclear lamina ( ${ }^{26}$ also Figure S1A). To identify lamin A-associated genome regions, we performed chromatin immunoprecipitation (ChIP) using the Strep-Tactin matrix for highly selective binding to the OST tag ${ }^{28-29}$ (Materials and methods; Figure S2A). Recovered chromatin was hybridized to NimbleGen promoter tiling arrays covering 2,500 bp promoter regions flanking the transcriptional start site of $~ 19,000$ annotated Refseq genes. Samples were run in duplicates and ChIPed DNA from empty vector infected cells, as well as input DNA from OST-A expressing cells, were used as

A

Target at nuclear lamina

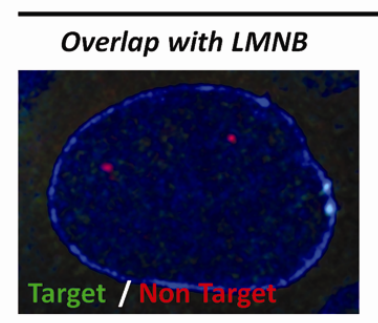

$<500 \mathrm{~nm}$ from LMNB

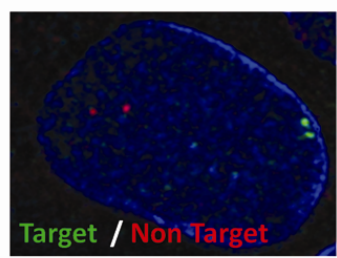

Target not at nuclear lamina

$>500 \mathrm{~nm}$ from LMNB

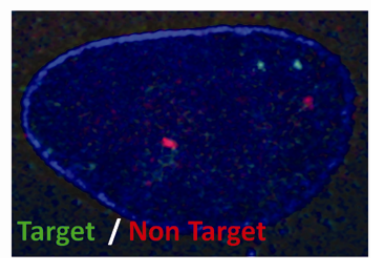

B

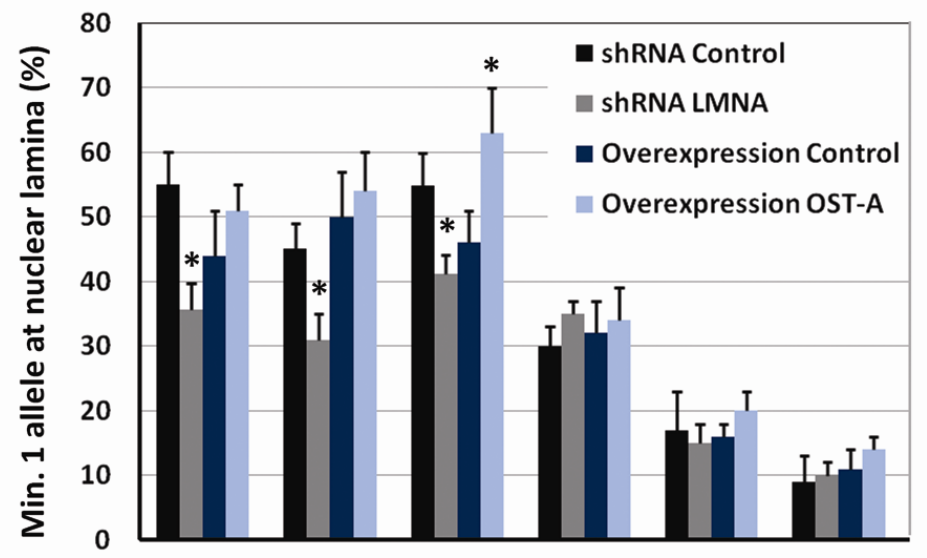

Acpp Sp100 Olfr681 Olfr1471 Eif2b Fanca

OST-A Targets

Non Targets

Figure 2. Subnuclear localization of lamin A-associated genes. (A) FISH on 4 lamin A targets (Acpp, Sp100, Olfr681, Olfr1471) and 2 non-targets (Eif2b, Fanca). The percentage of cells with at least one FISH signals near the nuclear lamina (overlapping or within $500 \mathrm{~nm}$ of lamin B) was measured. Typical examples of each scoring category are depicted for the lamin A target Sp100 (green), and the non-target Fanca (red). (B) Quantification of FISH staining for lamin A targets and non-targets in NkITAg cells infected with control shRNA, lamin A/C shRNA, OST-A or a control vector. Asterisks indicate a significant $(p<0.05 ; \chi 2$ test) change in peripheral positioning in comparison to control infected cells. 
controls (Figure S2A). 292 lamin A-associated genes were identified, based on enrichment in four independent experiments and stringent statistical criteria ${ }^{19}$ (Material and methods).

The 292 OST-A target genes localize to all chromosomes with the exception of the $\mathrm{Y}$ chromosome, which is absent in the female NkITAg cell line (Figure $1 \mathrm{~A}$ and S3). $27 \%$ of the 292 lamin A targets localize in gene clusters (defined

A

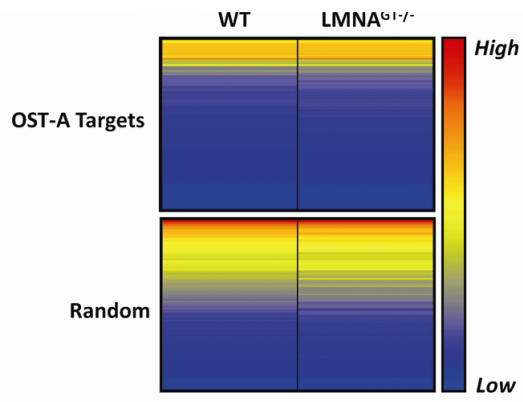

B

\begin{tabular}{|c|c|}
\cline { 2 - 2 } \multicolumn{1}{c|}{ B } & $\begin{array}{c}\text { Average Expression FC } \\
\text { LMNAGT- }- \text { vs. WT( } \pm \text { SD })\end{array}$ \\
\hline OST-A Targets & $1.03( \pm 0.12)$ \\
Random & $1.01( \pm 0.16)$ \\
\hline
\end{tabular}

C

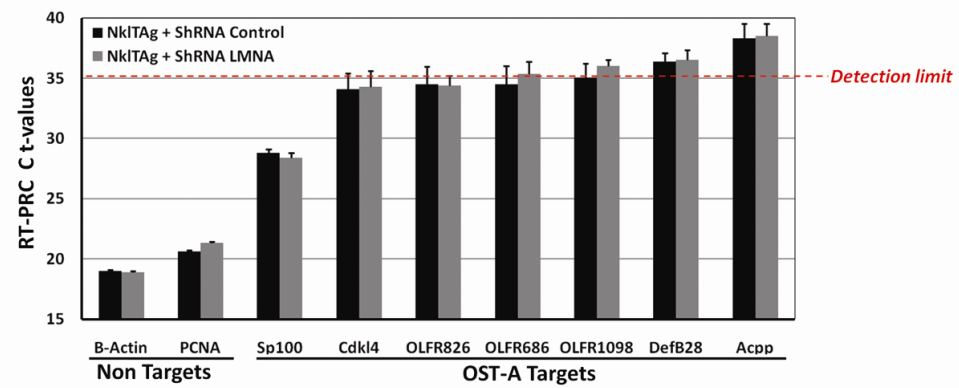

Figure 3. Expression profiles of lamin A-associated genes. (A) A genome-wide heatmap of RNA expression levels in cardiac left ventricle of wild-type (WT) and LMNA ${ }^{\mathrm{GT} /-}$ mice, which are functional knock-out for A-type lamins ${ }^{40}$. (B) Average fold changes ( $\pm s d$ ) of absolute linear RNA expression levels for lamin A targets and random gene sets upon loss of lamin $A / C$ (WT vs. LMNA ${ }^{\mathrm{GT}-\mathrm{F}_{-}}$mice). Observed fold changes did not differ significantly from 1.0 ( $p>0.05$; Mann-Whitney U test). (C) Real-time quantitative PCR on 7 lamin A targets (Sp100, Cdk/4, Olfr826, olfr686, olfr1098, Defb28 and Acpp) and 2 non-targets (B-Actin and Pcna) in the presence and absence of lamin A/C shRNA in NkITAg myocytes. Ct values ( $\pm s d$ ) are indicated, and reversely correlate to RNA expression levels (approximately a 2-fold decrease in expression levels per increased $\mathrm{Ct}$ value of 1 ). Ct values were all corrected for the housekeeping gene GAPDH expression levels and determined in hexaplo, using equal amounts of starting material (100ng cDNA) for each reaction. The detection limit ${ }^{41}$ of quantitative PCR (Ct value of $\sim 35$ ) is indicated with a dotted line. Ct values did not differ significantly ( $p>0.05$; Students T-test) for all targets between NkITAg cells infected with control and LMNA shRNA respectively. 
as two or more adjacent genes), which is 5 -fold higher ( $p<1.0 \times 10^{-4}$; Fisher's exact test) than expected for random gene association (Figure S4A and B). Gene ontology (GO) analysis identified 74 of the 292 target genes as involved in ' $G$ protein coupled receptor signaling' pathways (Figure 1B), with the vast majority $(\mathrm{N}=55)$ belonging to the $\mathrm{GO}$ subcategory 'perception of smell' including 52 olfactory receptors (OLFR) and 3 vomeronasal receptors (VMNR). This frequency is significantly higher $\left(p<1.0 \times 10^{-5}\right)$ than would be expected based on random association, which would predict less than 30 of the 292 lamin A-associated genes to be involved in this GO category. Other significantly enriched GO categories included 'locomotion' ( $N=11 / 292$, $\left.\mathrm{p}=1.3 \times 10^{-2}\right)$ and 'regulation of blood coagulation' $\left(\mathrm{N}=3 / 292, \mathrm{p}=3.5 \times 10^{-2}\right.$; Figure 1B).

Conservation plots using the Cis-regulatory Element Annotation System (CEAS) database ${ }^{22}$ did not reveal significant sequence conservation of precipitated DNA fragments (Figure S5), arguing against the existence of a specific lamin A-binding sequence. Analysis of known transcription factor binding motifs (TFM) by MatInspector software ${ }^{23}$ revealed 42 of the 170 known TFM to be significantly enriched in OST-A-associated gene promoters in comparison torandom promoter sequences $(p<0.05)$. The most highly enriched TFMs include those for the bromodomain and plant homeo domain transcription factor (BPTF, $p<1.0 \times 10^{-4}$ ) family, the three-amino acidloop extension (TALE, $\mathrm{p}<1.0 \times 10^{-4}$ ) and auto-immune regulator (AIRE, $p<1.0 \times 10^{-4}$ ) TF families. These data demonstrate that A-type lamin interacting gene promoters preferentially localize in genomic clusters, lack unique consensus lamin A binding sequences but are enriched for several transcription factor binding motifs.

\section{Nuclear localization of lamin A-associated genes}

To assess the subnuclear localization of lamin A-associated genes and to validate the ChIP technique, the location of 4 lamin A targets and 2 nontargets in NkITAg cells were probed by DNA FISH. We measured the percentage of cells with at least one FISH signal within $500 \mathrm{~nm}$ of the nuclear periphery identified by lamin B (material and methods; Figure $2 \mathrm{~A}$ ) ${ }^{30}$. 
For the randomly selected lamin A targets Acpp, Sp100, Olfr681 and Olfr1471 located on chromosomes 9, 1, 7, and 19, respectively, at least one allele localized to the periphery in $55,45,55$ and $30 \%$, respectively, of cells compared to 17 and 9\% for the non-targets Eif2b and Fanca (Figure 2B). The difference between peripheral localization of lamin $A$ targets and nontargets is statistically significant at $p<0.001$ in a $\chi^{2}$ test.Next we set out to probe the effects of loss of $A$-type lamins on the subnuclear localization of lamin $A$ associated-genes by knocking-down lamin $A / C$ using shRNA in NkITAg cells. Expression of lamin A/C shRNA resulted in over $90 \%$ knockdown for both lamin $A$ and $C$ proteins (Figure S1B). The peripheral localization of the lamin A target loci Acpp, Sp100 and OLFR681 decreased significantly to 35,31 and $25 \%$ ( $p<0.01, \chi^{2}$ test; Figure $2 B$ ). In contrast, the lamin-associated OLFR1471 locus and the non-targets Eif2b and Fanca retained their subnuclear position ( $p>0.05, \chi^{2}$ test; Figure $2 B$ ). In contrast, overexpression of OST-A at endogenous levels did not affect localization of lamin A-associated genes, with the exception of the lamin A target OLFR681, which showed increased localization to the nuclear lamina upon introduction of OST-A (from $46 \%$ to $63 \%, p<0.05, \chi^{2}$ test). These findings support a role for lamin $A$ in recruiting gene promoters to the nuclear periphery.

\section{Expression profile of lamin A-associated genes}

To obtain insights into the effect of lamin A/C and its loss on lamin A target genes in NkITAg cardiac myocytes, we analyzed the expression profiles of lamin $A$ interacting genes using microarray expression profiles of neonatal cardiac tissues of WT and LMNA ${ }^{\text {GT- }-}$ mice, a LMNA null model based on gene trap technology (Kubben et al., in preparation). Basal expression levels of lamin A-interacting genes are significantly lower compared to random gene sets $(\Delta \log 2-1.28, p<0.001$, Mann-Whitney $U$ test; Figure $3 A)$. Amongst the 292 lamin A-associated target genes, 238 genes were not expressed or expressed at very low levels (absolute linear expression value $<25$, which is 650 -fold lower than the housekeeping gene GAPDH), 18 were expressed at low levels (linear expression value 25-100) and 36 had roughly average expression levels (linear expression value $>100$ ). 
Loss of A-type lamins in LMNA ${ }^{\text {GT-F }}$ cardiac tissue significantly changed the transcriptional profile of 1136 genes (Kubben et al., in preparation). The average expression fold change (FC) of the 292 lamin Aassociated genes was similar to the FC observed in the total genome, and indicated no preferential effect of lamin A loss on the lamin A-associated genes (OST-A targets FC 1.03 $\pm 0.12, p=0.604$; Random genes FC 1.01 $\pm 0.16, P=0.478$, MannWhitney $U$ test; Figure $3 \mathrm{~B})$. As these genes lose their association with the lamina (Figure 2B), absence of any effect on gene transcription argues that re-activation/expression isprobably not dependent on interaction with lamin A or the lamina.

Microarray data were confirmed by quantitative real-time PCR on seven lamin A targets (Sp100, Cdk14, Olfr826, Olfr686, Olfr1098, Defb28 and Acpp) and two non-targets (B-actin, Pcna) in NkITAg cardiac myocytes. All lamin A targets were expressed at low levels near the detection limit of the RT-PCR (Ct values in the range of 33-38), with the exception of $\operatorname{Sp100}\left(C_{t}\right.$ values of 29 ), in contrast to the non-targets $B$-actin and Pcna ( $C_{t}$ values of 18-21; Figure $3 C$ ). Knock-down of lamin $A / C$ did not result in significant changes in expression levels for the tested genes ( $p>0.05$, Student's T-test) in accordance with the expression microarrays results. Combined, these findings support a role for lamin A in nuclear organization of chromatin through association with transcriptionally silent genes. Our data also suggest that loss of association of genes with A-type lamins is not sufficient for gene activation.

\section{Identification of lamin-A and progerin associated genome regions}

We next sought to identify genome regions which interact uniquely with lamin $A$, uniquely with progerin or with both. To this end OST tagged lamin A (OST-A) or progerin (OST-P) were expressed at endogenous levels in wildtype (WT) or LMNA knock-out (LMNA ${ }^{\mathrm{KO} / /}$ ) MEFs. As previously shown, and in contrast to OST-A, OST-P expression leads to distortions of the nuclear lamina (Figure S1A) and global loss of LAP2 and HP1Y nuclear levels ${ }^{26}$ as observed in HGPS patient cells ${ }^{5}$. Lamin A-associated gene promoters were first identified by hybridizing ChIPed chromatin of WT or LMNA ${ }^{\mathrm{KO}-{ }_{-}}$MEFs expressing OST-A against that of empty vector infected cells (Figure S2B). 
A

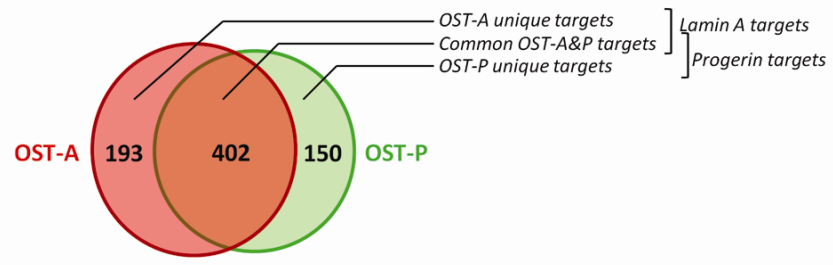

B

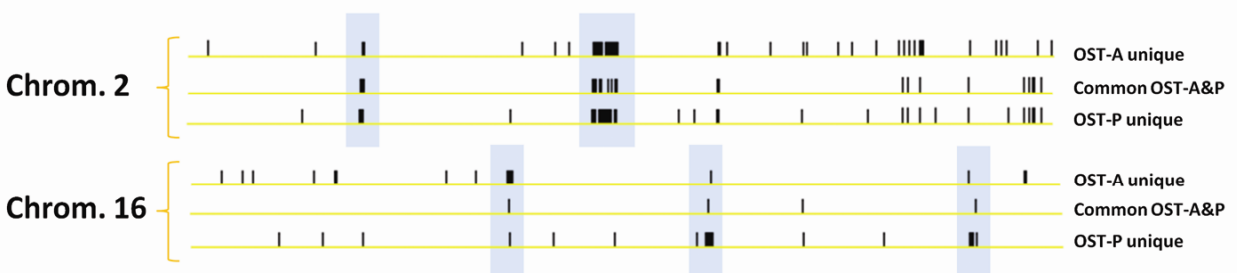

\begin{tabular}{|c|c|c|c|c|}
\hline $\begin{array}{l}\text { CC Target } \\
\text { Genes }\end{array}$ & GO Category & $\begin{array}{c}\text { N / All } \\
\text { CCtargets }\end{array}$ & $\begin{array}{c}N / \text { All CC } \\
\text { targets for } \\
p=0.05\end{array}$ & $P$ value \\
\hline \multirow{4}{*}{ OST-A unique } & G protein coupled receptor signaling & $27 / 193$ & $21 / 193$ & $1.7 \times 10^{-2}$ \\
\hline & Cation Transport & $16 / 193$ & 9/193 & $7.4 \times 10^{-5}$ \\
\hline & Response to cytokine stimulus & $5 / 193$ & 2/193 & $1.8 \times 10^{-4}$ \\
\hline & Regulation of hormone levels & 7/193 & $4 / 193$ & $1.2 \times 10^{-3}$ \\
\hline \multirow{3}{*}{$\begin{array}{l}\text { Common } \\
\text { OSTA\&P }\end{array}$} & G protein coupled receptor signaling & $157 / 402$ & $42 / 402$ & $7.8 \times 10^{-71}$ \\
\hline & Defense response to bacteria & $12 / 402$ & $6 / 402$ & $7.2 \times 10^{-5}$ \\
\hline & Response to pheromone & $11 / 402$ & $5 / 402$ & $2.6 \times 10^{-5}$ \\
\hline \multirow{2}{*}{ OST-P unique } & G protein coupled receptor signaling & $27 / 150$ & $16 / 150$ & $9.7 \times 10^{-4}$ \\
\hline & Regulation of translation & $4 / 150$ & $3 / 150$ & $3.9 \times 10^{-2}$ \\
\hline
\end{tabular}

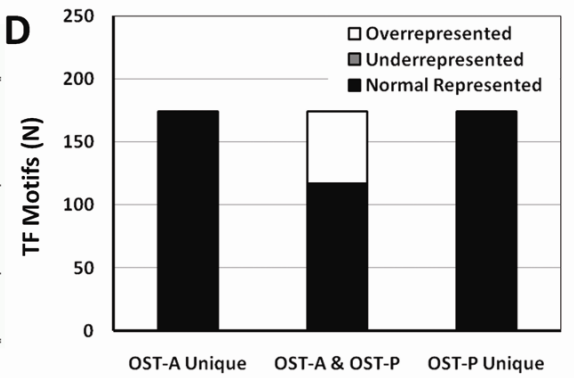

Figure 4. Genomic localization and characteristics of common and differential lamin A- and progerinassociated genes. (A) Venn-diagram of lamin A- and progerin-interacting genes determined by ChIP-chip in MEF cells (See Material \& Methods). Overlapping targets are referred to as common OST-A\&P targets, while genes only interacting with lamin A or progerin, are named OST-A unique and OST-P unique targets respectively. (B) A genomic plot of OST-A unique, OST-P unique and common OST-A\&P subsets of targets on chromosome 2 and 16. Boxes highlight examples of genomic areas containing gene targets from all 3 subsets (on chromosome 2), and regions that preferentially represent gene targets from one subclass (on chromosome 16). (C) Summary of gene ontology (GO) analysis on gene targets of all 3 subsets, indicating the GO class, the amount of genes $(\mathrm{N})$ per total ChIP target genes in that GO class, the minimal amount of targets needed for a significance level of 0.05 and the $p$-value, based on statistical testing fully described previously ${ }^{39}$. (D) ChIPed DNA fragments of all 3 subclasses were analyzed for the occurrence of 170 transcription factor motifs (TFM) (See Material \& Methods). Common OST-A\&P targets were shown to be enriched for 58 TFM, while all 170 TFM were normally represented in OST-Aunique and OST-P unique targets subsets. 
Progerin-interacting gene promoters, as well as differences in lamin A and progerin-gene promoter interaction profiles, were then determined by comparing OST-P ChIPed chromatin with OST-A ChIPed chromatin as a control (Material and methods; Figure S2B). We identified 595 lamin Aassociated gene promoters (defined as promoters interacting with OST-A in both WT and LMNA ${ }^{\mathrm{KO}-/-} \mathrm{MEFs}$ ) and 552 progerin-associated gene promoters (defined as promoters interacting with OST-P in both WT and LMNA ${ }^{\mathrm{KO}-/}$ MEFs) (Figure 4A, S2B). A significant overlap was present for lamin Ainteracting genes $(n=595)$ between WT $(N=1686)$ and $L_{M N A}{ }^{K O-}$ MEFs $(\mathrm{N}=840)\left(\mathrm{p}<1 \times 10^{-3}, \chi^{2}\right.$ test), as well as for progerin-interacting genes $(\mathrm{n}=552)$ in WT $(\mathrm{N}=1214)$ and $\mathrm{LMNA}^{\mathrm{KO}-/}$ MEFs $(\mathrm{N}=1642)\left(\mathrm{p}<1 \times 10^{-3}, \chi^{2}\right.$ test) . Laminassociated genes identified in MEFs and NkITAG cells significantly overlapped as $23 \%$ of the 595 lamin A target genes, and $23 \%$ of the 552 progerin target genes identified in MEFs were also identified at the nuclear lamina in at least one ChIP-on-chip experiment in NkITAg cells as well $\left(p<1 \times 10^{-3}, \chi^{2}\right.$ test $)$.

Over two-thirds ( $\mathrm{N}=402)$ of the identified lamin-associated genes in MEFs interacted with both lamin $A$ and progerin, and will further be referred to as common OST-A\&P targets (Figure 4A). 193 lamin A target genes interacted uniquely with lamin A (OST-A unique targets) and 150 target genes interacted specifically with progerin only and were not enriched in OST-A ChIP experiments in the absence of progerin (OST-P unique targets). Closer examination of the genomic localization reveals that $38 \%$ of the lamin A- as well as the progerin-associated genes occurs in clusters (varying in size from 2 to 11 adjacent genes), which is 5 -fold more frequent than expected for random gene sets $\left(p<1 \times 10^{-4} ;\right.$ Fisher's exact test), and both sets of interactors overlap significantly $\left(p<1 \times 10^{-10}\right.$; Figure S4 C-F). Despite this genomic colocalization of lamin A- and progerin-associated genes, occasional genomic regions that preferentially bind to progerin or lamin $A$ could be found, for instance on chromosome 16 (Figure 4B).

GO analysis identified 157 of the 402 common lamin A and progerin targets involved in ' $G$ protein coupled receptor signaling' pathways (Figure $4 C$ ). This frequency is significantly higher $\left(p<1.0 \times 10^{-5}\right)$ than would be expected based on random association of genes, which predicts less than 42 of 402 associated genes to be involved in this GO category. Other GO categories 
that were significantly enriched, include 'Defense response to bacteria' $\left(12 / 402, p=7.2 \times 10^{-5}\right)$ and 'Response to pheromone' $\left(11 / 402, p=2.6 \times 10^{-5}\right)$. The former category mainly consists of defensin and defensin-related cryptdin proteins, and the latter of vomeronasal receptors, which are also represented in the ' $G$ protein coupled receptor signaling' $G O$ class. In comparison to common OSTA\&P targets, lamin A uniquely associated genes were significantly less enriched in the ' $G$ protein coupled receptor signaling' category $\left(27 / 193, p=1.7 \times 10^{-2}\right.$; Figure $\left.4 C\right)$, but instead were enriched in 'cation transport' $\left(16 / 193, p=7.4 \times 10^{-5}\right)$, 'response to cytokine stimulus' $\left(5 / 193, p=1.8 \times 10^{-4}\right)$ and 'regulation of hormone levels' $\left(7 / 193, p=1.2 \times 10^{-3}\right)$. OST-P unique targets were enriched for ' $G$ protein coupled receptor signaling' $\left(27 / 150, p=9.7 \times 10^{-4}\right)$ and 'regulation of translation' (4/150, $p=3.9 \times 10^{-2}$ ). The enrichment of different GO families amongst OST-A, OST-P and OSTA\&P targets suggests distinct preferential association of various gene groups with lamin A or progerin.

As for lamin A- and progerin-interacting genes, no consensus lamin binding sequence could be identified in any subclass of target genes (Figure S5). TFM analysis indicated enrichment of common lamin A/progerin-associated gene promoters for 58 TFMs ( $p<0.05$, Figure 4D, Table S2). Over half of these TFMs were also found enriched in lamin A targets within the NkITAg cardiac myocytes, including the 5 most significantly enriched TFs brain- 5 (BRN5), serum responsive factor (SRF), octamer transcription factor 1 (Oct1), bromodomain and plant homeo domain transcription factor (BPTF) and HOXC (all $p<1 \times 10-5$, Table S2). Lamin A unique and progerin unique targets contained TFM (170 analyzed) at similar frequencies as random promoter sequences $(p>0.05)$. Overall these data show that lamin $A$ and progerin interact differently with chromatin, and that common and uniquely lamin Aor progerin-associated genes possess distinct features including their biological function and TFMs.

\section{Subnuclear localization of common versus unique lamin A- and progerin-associated genes}

To assess the subnuclear localization of common and unique lamin A- and progerin-associated genes, four randomly selected target genes for each subset (OST-A unique: Eef1a2, Kcnip2, Scn5a, Sdro; common OST-A\&P: 
Cyp3a25, Olfr804, ABPD, Defcr5; OST-P unique: Daf2, Tcf21, Trim30, $A b c b 10)$ were analyzed by FISH. Peripheral localization was assigned to FISH signals when at least one allele per cell overlapped with the nuclear periphery, defined by lamin B staining (Figure 5A). In WT MEFs cells infected with an empty vector, the non-target $E 1 f 2 b$ only sporadically localized peripherally (1\%), while for unique lamin $A$ target genes the frequency of lamin B signal overlap varied from $4 \%$ (Eef1a2) to $29 \%$ (Scn5a), for progerin unique interacting genes from $2 \%(D a f 2)$ to $23 \%(A b c b 10)$ and for common targets frequencies were between 53\% (Cyp3a25) and 65\% (ABPD) (Figure $5 B)$. Overall, these data indicate that common lamin $A /$ progerin targets showed an overall significantly higher frequency of lamin B signal overlap (58\% on average) than OST-A unique (17\% average) and OST-P unique targets (16\% average) (Figure 5B, Table S4; Pvalue<0.001, $\chi^{2}$ test). This preferred peripheral localization of common lamin $A$ /progerin-associatedgenes was independent of the presence of endogenous A-type lamins as common interactors in empty vector infected $\mathrm{LMNA}^{\mathrm{KO}-\mathrm{-}}$ MEFs also more frequently overlapped with lamin B (47\% average, range $43-54 \%)$, in comparison to lamin A (19\% average, range $8-39 \%)$ and progerin $(11 \%$ average, range 5-15\%) uniquely associated genes (Table S4; Pvalue $<0.001, \chi^{2}$ test).

Expressing OST-A at endogenous levels in LMNA ${ }^{\mathrm{KO}-/}$ MEFs affected subnuclear positioning of unique lamin A targets, as Eef1a2, Kcnip2 and Scn5a, but not Sdro showed increased peripheral localization (OST-A unique targets; $p<0.05, \chi^{2}$ test; Figure $5 B$ ). In contrast all common lamin $A /$ progerin interactors (Cyp3a25, Olfr804, Abpd, Defcr5) as well as progerin unique interactors (Daf2, Tcf21, Trim30, Abcb10) examined, were insensitive to higher levels of OST-A and did not change subnuclear positioning (all $p>0.05$, $\chi^{2}$ test). In WT MEFs, introduction of OST-A significantly increased peripheral localization of the lamin A uniquely associated gene Kcnip2, but not of the other probed loci. These results suggest that the subnuclear positioning of uniquely lamin A-interacting genes is sensitive to the levels of lamin A, in contrast to genes which interact both with progerin or both lamin $A$ and progerin.

Expression of OST-P at endogenous levels in LMNA ${ }^{\mathrm{KO}-/}$ MEFs specifically influenced the peripheral positioning of the progerin uniquely-associated 
No overlap with $L M N B$
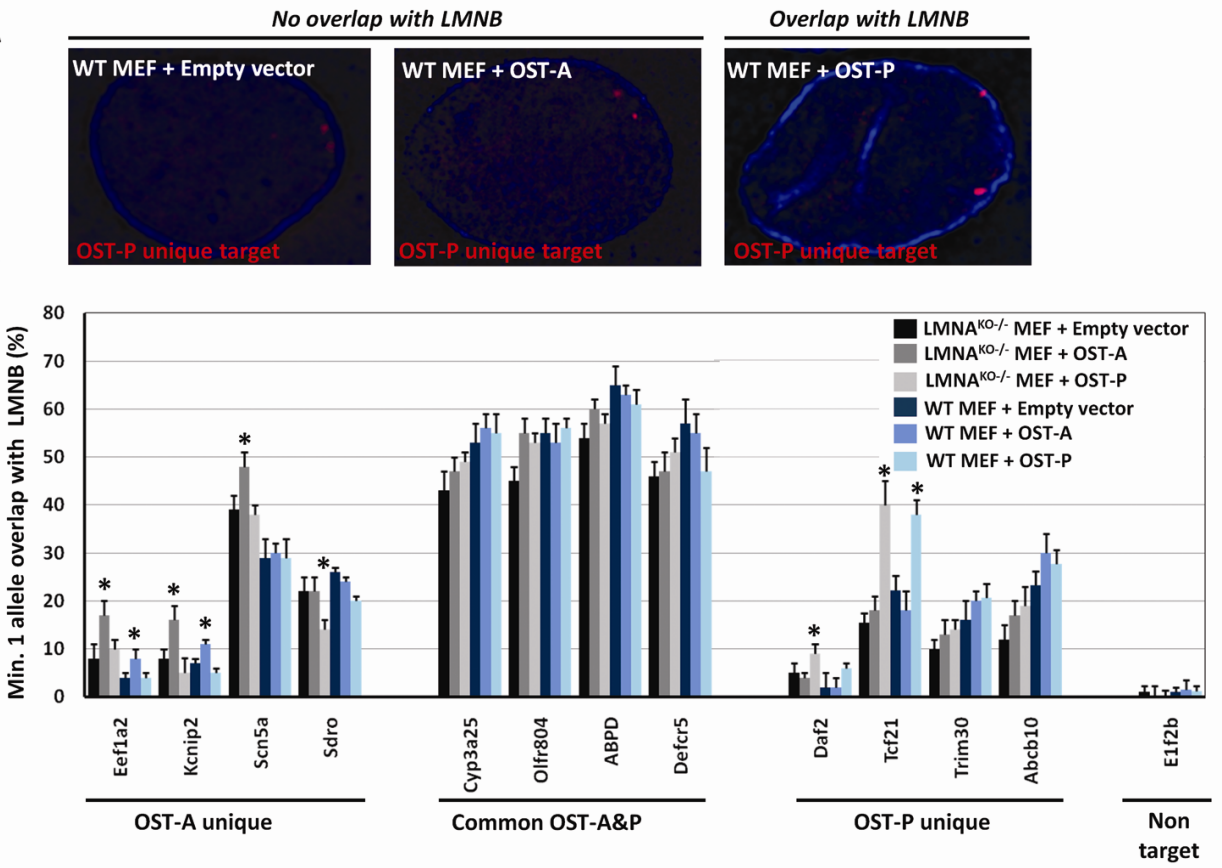

Figure 5. Subnuclear positioning of common and differential lamin A- and progerinassociated genes. (A) FISH was performed on 4 loci per subset of ChIP targets (OST-Aunique: Eef1a2, Kcnip2, Scn5a, Sdro; common OST-A\&P: Cyp3a25, OLFR804, ABPD, Defcr5; OSTPunique: Daf2, Tcf21, Trim30, Abcb10) as well as one non-target (E1f2b). An example is shown of the Tcf21 gene in wild-type (WT) MEFs expressing OST-A, OST-P or an empty control vector. Signals (red) were scored to overlap with lamin B (blue) at the nuclear rim or not. (B) FISH quantification of the frequency of cells with minimally one FISH signal overlapping with lamin B, indicated for all ChIP targets in A-type lamin knock-out (LMNAKO-/) MEFs and WT MEFs, expressing OST-A, OST-P or an empty control vector. Significant differences in localization with the corresponding empty vector control cells are indicated by an asterisk ( $p<0.05 ; \chi 2$ test).

genes, as Daf2 and $T c f 21$ became more frequently peripherally localized (OST-P unique targets; $P<0.05, \chi^{2}$ test; Figure $5 B$ ). In addition, an opposite effect was observed for the lamin A unique interactor Sdro, which significantly lowered its peripheral localization in the presence of progerin $\left(p<0.01, \chi^{2}\right.$ test; Figure $\left.5 B\right)$. All other investigated unique lamin $A-$ and common lamin $A$ /progerin-associated loci maintained their subuclear position. Interestingly, the introduction of OST-P in a WT MEF background, similar to the situation found in heterozygous HGPS patients, increased 
peripheral localization of the Tcf21 progerin uniquely interacting gene, but none of the other loci tested, suggesting that the presence of progerin in a wild-type background may have gene-specific effects, but does not dramatically alter the location of genes globally.

For the lamin A unique interactors expression of OSTA in LMNA ${ }^{\mathrm{KO}-{ }^{-}}$MEFs increased the relative frequency of lamin B signal overlap by $54 \%$ on average, which is significantly ( $p<0.05$, Student's T-test; Table S3) different from the $17 \%$ decrease observed upon the introduction of progerin. In WT MEFs these differences were less pronounced, $+38 \%$ upon lamin A and $-13 \%$ upon progerin expression ( $p=0.08$, Student;s T-test; Table S3). These results suggest that lamin A contributes significantly to the peripheral localization of lamin A-unique interactor sequences.

For uniquely progerin-associated genes, the increased peripheral localization upon the introduction of OST-A in LMNA ${ }^{\mathrm{KO}-1-}$ MEFs $(+18 \%$ on average), was significantly less pronounced than upon the expression of OST-P (+83\%; Table S4; $p<0.05$, Student's T-test). In WT MEFs these differences, $+10 \%$ and $+106 \%$ upon lamin $A$ or progerin expression, respectively, were borderline significant ( $p=0.09$, Student's T-test; Table S3).

Common targets on average did not change $(p>0.05)$ their peripheral positioning upon the addition of either OST-A or OST-P in either LMNA ${ }^{\mathrm{KO}-/}$ MEFs (+11\% OST-A; $+11 \%$ OST-P) or WT MEFs (-1\% OST-A; $-3 \%$ OST-P). Overall, these data demonstrate that common lamin A/progerin interacting genes differ from unique interactors by an increased peripheral positioning, which is relatively insensible to nuclear lamin A or progerin protein levels. In contrast, unique lamin $A$ and progerin targets can further be distinguished from each other in that they specifically increase their peripheral localization upon the expression of OST-A and OST-P respectively.

\section{Global gene expression profiles of common versus unique lamin A- or progerin-associated genes}

To examine the expression behavior of lamina-associated genes in response to overexpression of lamin A or progerin, we analyzed mRNA from WT MEFs transfected with OST-A, OST-P or an empty vector on expression arrays. As 
previously observed in other systems ${ }^{31}$, introduction of progerin changed the global transcriptional profile significantly by up-regulating 468 genes and downregulating 702 genes ( $p<0.05$; Figure 6A). OST-A expression affected 1142 genes (571 up and 517 down) of which 379 targets were also affected by expression of OST-P.

When compared to empty vector controls, lamin A, progerin and common targets generally show lower basal expression levels than random sets of genes ( $\log _{2}$ FC $-1.9, p<1 \times 10^{-3}$, Mann-Whitney U-test; Figure 6B). In good agreement with their localization at the lamina, common targets were most repressed compared to random genes $\left(\Delta \log _{2}-2.6, \mathrm{P}<1 \times 10^{-3}\right.$, Kruskall-Wallis test) followed by lamin $A$ unique targets $\left(\log _{2}-2.2 ; \mathrm{P}<1 \times 10^{-3}\right.$, Kruskal-Wallis test) and progerin unique targets $\left(\log _{2}-1.1 ; p<1 \times 10^{-3}\right.$, Kruskal-Wallis test). mRNA levels of all three individual classes differ significantly from each other ( $p<1 \times 10^{-3}$, Kruskal-Wallis test). These basal expression levels of the subclasses did not change significantly upon OST-A or OST-P expression in WT MEFs ( $p>0.05$, Kruskall-Wallis Test; Figure 6C), suggesting that expression of lamin A or progerin is not sufficient to induce a global change in gene activity of lamina-associated genes. Overall, these data indicate that, independent of the presence of progerin, common lamin A/ progerin targets have lower basal expression levels than lamin $A$ or progerin unique targets, which appear to partially escape the repressive effect of the NE.

\section{Progerin-induced changes in gene expression of lamina-associated genes}

To further probe the effect of progerin on lamina-associated genes, we analyzed individual ChIP targets whose expression were affected by the expression of progerin and not lamin A. 53 of 745 genes associated with lamin $A$ and/or progerin display a significantly altered expression (Table S5) upon introduction of OST-A or OST-P into WT MEFs. 28 of 53 genes respond only to progerin and not lamin A (relative expression changes $-27 \%$ to $+63 \%$ for OST-P; Table S5), 16 change expression upon lamin A but not progerin introduction (-11 to $+36 \%$ for OST-A; Table S5), and 9 genes respond to both progerin and lamin $A$, of which one (Serpin1ad1) shows an opposite response to both lamins (-13\% for OST-A; $+11 \%$ for OST-P), 3 are downregulated equally by both lamins (Fcer2a, Smarca5, Al747699; range $-4 \%$ to - 
$46 \%$ for OST-A or OST-P), 2 show decreased mRNA levels upon the expression of lamin A (Hoxc11- 4\%, Srp9 -17\% for OST-A) and further decreased levels upon the introduction of OST-P (-13 and $-26 \%$, respectively), and the remaining 3 are down-regulated by progerin (Cysltr1 1\%, Abpg $-6 \%$ and 5730494 M16Rik -9\% for OST-P) and more pronounced by

A

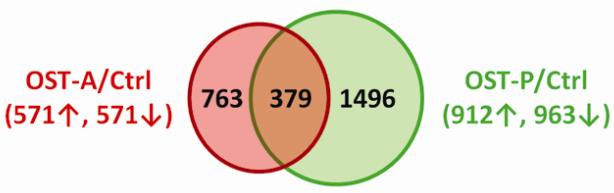

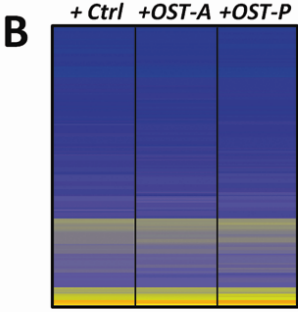

OST-A Unique

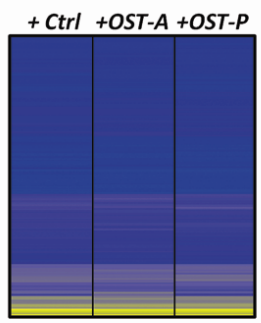

Common OST-A\&P

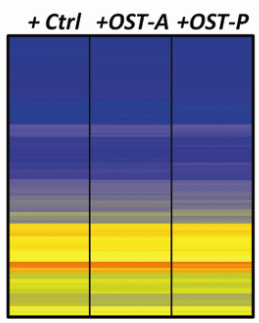

OST-P Unique

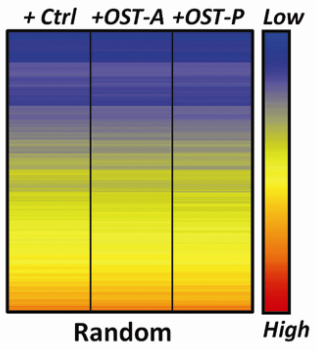

C

\begin{tabular}{|c|c|c|c|c|}
\hline & \multicolumn{3}{|c|}{ Average Expression FC ( \pm SD) } \\
\hline & & $\begin{array}{l}\text { MEF OST-A } \\
\text { vs. } \\
\text { MEF Empty }\end{array}$ & $\begin{array}{l}\text { MEF OST-P } \\
\text { vs. } \\
\text { MEF Empty }\end{array}$ & $\begin{array}{l}\text { MEF OST-P } \\
\text { vS. } \\
\text { MEF OST-A }\end{array}$ \\
\hline \multirow{4}{*}{ 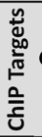 } & OST-A Unique & $1.00( \pm 0.10)$ & $1.01( \pm 0.10)$ & $1.00( \pm 0.11)$ \\
\hline & Common OST-A\&P & $1.01( \pm 0.09)$ & $0.98( \pm 0.07)$ & $0.98( \pm 0.09)$ \\
\hline & OST-P unique & $0.99( \pm 0.09)$ & $0.98( \pm 0.12)$ & $0.98( \pm 0.09)$ \\
\hline & Random & $1.00( \pm 0.09)$ & $0.99( \pm 0.12)$ & $0.99( \pm 0.07)$ \\
\hline
\end{tabular}

Figure 6. Expression profiles of common and differential lamin A- and progerin-associated genes. (A) A Venn diagram depicting the amount of genes which are deregulated $(p<0.05$, Anova; no minimal fold change cut-off used) upon introduction of respectively OST-A or OST$P$ in WT MEFs. The amount of genes up( $\uparrow)$ - and down $(\downarrow)$-regulated are indicated. (B) Expression heatmaps of lamin $\mathrm{A} /$ progerin unique and common targets and a random gene set. Expression levels are indicated for WT MEFs expressing OST-A, OST-P or an empty control vector. Common interactors have lower expression levels compared to lamin $A$ and progerin unique interactors $(\mathrm{P}<0.001$; Kruskall-Wallis test). Unique interactors have lower basal expression levels compared to random genes ( $p<0.001$; Kruskall-Wallis test). (C) Average expression fold changes (FC) ( \pm sd) are given for all 3 subsets of gene targets, comparing WT MEFs expressing OST-A or OST-P with empty vector cells and with each other. Fold changes are based on absolute linear expression values and all were insignificant ( $p>0.05$; Kruskall-Wallis test). 
lamin A (-5, -8 and $-15 \%$ respectively). Despite these significant changes, expression levels for 44 of 53 targets remain very low (absolute linear expression value 1-50, which is 325 -fold lower than the housekeeping gene GAPDH). Four genes have moderate expression levels (50-100), and increase their expression levels either by either progerin (B130050I23Rik $+20 \%$ and Krtap5-1 +27\% for OST-P), or lamin A (Pnpla $2+17 \%$ for OST-A) or decrease expression by both lamins (Srp9, -17\% for OST-A, -26\% for OST-P). 5 genes were more highly expressed $(>100)$, of which two change expression levels upon the expression of OST-P, but not OST-A ( $\mathrm{Cmtm} 3+19 \%$, Rpn $2+3 \%$ for OST-P), 5730494M16Rik changes expression upon the introduction of OST-A (-5\%), but not OST-P, and two genes are down-regulated by both lamins, of which Smarca5 responds equally to both lamins (-44\% for OST-A, $-33 \%$ for OST-P) and 2700069A02Rik is down-regulated more drastically by lamin A than progerin (-15\% for OST-A, $-9 \%$ for OST-P). Overall, these data identify novel lamina-associated genes whose expression is specifically deregulated by progerin, lamin A or both.

\section{Discussion}

The presence of chromatin defects in several laminopathies ${ }^{8}$ points to an important role for A-type lamins in chromatin organization. Recent findings ${ }^{16}$ indicate direct lamin A-chromatin interactions are critical for such chromatin organization and suggest that aberrant chromatin-lamin interactions may contribute to laminopathies. We here map genes which interact with lamin A or with the disease causing lamin $A$ isoform progerin in a genome-wide, unbiased fashion. We find that lamin A preferentially binds to peripherally localized, genomically clustered and silent genes, and that chromatin interactions for progerin and lamin A are substantially different. Furthermore, we demonstrate that loss or gain of interaction with lamin A or progerin changes the nuclear position of many interacting genes, but per $s e$ is not sufficient to change gene expression levels of the interacting genes. We speculate, however, that altered gene location may predispose genes for subsequent aberrant regulation.

Recent studies using the DamID method to map lamin-interacting genome regions ${ }^{12-13}$ have revealed the importance of lamina-chromatin interactions 
in chromatin organization and gene expression regulation by identifying sharply defined lamin-associated domains (LADs) across the genome ${ }^{11}$. LADs are preferentially localized in gene-poor, low expressed regions, yet they contain cell-type specifically silenced gene clusters ${ }^{32}$. In agreement, using an independent approach we find that lamin A-associated genes are generally silent or very lowly expressed. Lamin A target genes also show preferential localization at the nuclear periphery compared to non-targets and are genomically clustered. Consistent with this, $\mathrm{GO}$ analysis reveals that lamin A-associated genes are enriched in gene groups such as sensory perception of smell and bacterial response which are not functional in the fibroblasts or cardiomyocytes analyzed here, and are hence expected to be transcriptionally silent in these cells ${ }^{33-34}$. The presence of over 42 tissue and cell-type specific transcription factor binding motifs (TFMs) in lamin Aassociated gene promoters further supports this notion. Other enriched TFMs, like RP58, may contribute to the transcriptional repressive environment of the NE by helping maintain condensed chromatin ${ }^{35-36}$.

We identified targets which associate with lamin A or with progerin or with both. The former two classes of differential targets are distinguished from common targets by a number of characteristics: First, unique targets are less involved in cell-type specific biological processes and are not enriched for any of the 172 investigated TFM. Second, lamin A/progerin common target genes are more frequently co-localized with lamin $B$ at the nuclear lamina than lamin A or progerin unique targets. Correspondingly, we find that the peripheral position of common targets is significantly less affected by overexpression of lamin A or progerin than that of unique targets. Finally, genome-wide expression profile analysis shows that regardless of the presence of progerin, basal expression levels of common lamin A and progerin targets are lower than those of lamin A or progerin-unique targets. These data are in good agreement with our FISH analysis and suggest that transcriptional silencing of common targets at the nuclear lamina is sustained, whereas unique targets partially escape interaction with the lamina under normal conditions, and are likely less influenced by repressive capacities of the NE. The observation that in the absence of progerin, uniquely lamin A -associated genes show significantly lower expression than progerin-specific interacting targets, which in this state do not interact with the lamina, further corroborates this notion. 
To determine the importance of lamin-chromatin interactions in subnuclear positioning and regulation of gene expression, we applied FISH and expression array technology to lamin A loss- and gain-of-function cell models. RNAi-mediated knock-down of lamin A/C reveals substantial relocalization of lamin A target loci to the nuclear interior. Despite this relocalization, lamin A-associated genes remain overall more frequently localized at the NE compared to non-targets. The reverse experimental approach, in which we overexpressed lamin $\mathrm{A}$ in $\mathrm{LMNA} \mathrm{KO}-\%^{\mathrm{KO}} \mathrm{MEFs}$, lead to the movement of several lamin $A$ target genes to the nuclear periphery. Introduction of progerin in LMNA ${ }^{\mathrm{KO}-/-}$ MEFs increased the peripheral position of progerin unique targets and resulted in a repositioning of lamin $A$ targets towards the nuclear interior. Combined, these findings support a facilitating role for A-type lamins in recruitment of silenced genes to the nuclear periphery. Interactions with additional nuclear lamina proteins, like emerin and lamin B, which are known chromatin interactors ${ }^{11,}{ }^{13}$, likely contribute to this functional nuclear organization.

A key outcome of our analyses is the notion that while localization of (silent) genes to the periphery is dependent on lamin A, dissociation from the lamina does not necessarily lead to their activation. We speculate that loss of lamina-association is only one of multiple steps required for gene activation. Consistent with this, the relocation of the CFTR locus due to activation and consequent internalization of neighboring genes by itself is insufficient for CFTR activation ${ }^{37-38}$. Furthermore, for olfactory receptors, which were identifiedas the most prominent group of lamin A targets, many additional steps necessary for stochastic activation have been described as well ${ }^{34}$. It is possible that dissociation from the periphery renders the transcriptional status of these genes to "poised" and facilitates their activation by subsequent signals that may occur e.g. during differentiation. Extending these studies to differentiation models in which additional factors support gene activation of specific subsets of target genes are active will be important. The herein described identification of specific lamin A and/or progerin interacting genes provides a basis for further study of the role of lamina-chromatin dissociation in transcriptional activation and the etiology of laminopathies. 


\section{Acknowledgements}

We express our gratitude to K. Meaburn and T. Karpova for their help in setting up FISH protocols and imaging software. O. Hakim, E. Olson and L. de Windt are thanked for sharing cell lines and reagents. We thank $A$. van Erk and L. Eijssen for helping with analysis of expression microarrays and their help in creating software to plot the genomic localizations of ChIP targets. $R$. van Leeuwen, L. van Opstal and C. Calis for their help in optimization of the ChIP protocol. This research was supported in part by the Intramural Research Program of the National Institutes of Health (NIH), NCl, Center for Cancer Research, grants from the Dutch Heart Foundation, ZonMW and the EU-KP7 grant 'Inheritance'. 


\section{References}

1. Broers JL, Ramaekers FC, Bonne G, Yaou RB, Hutchison CJ. Nuclear lamins: laminopathies and their role in premature ageing. Physiol Rev 2006; 86:967-1008.

2. Hutchison CJ. Lamins: Building blocks or regulators of gene expression. Nature Reviews Molecular Cell Biology 2002; 3:848-58.

3. Broers JL, Hutchison CJ, Ramaekers FC. Laminopathies. J Pathol 2004; 204:478-88.

4. Sullivan T, Escalante-Alcalde D, Bhatt $H$, Anver M, Bhat N, Nagashima K, et al. Loss of A-type lamin expression compromises nuclear envelope integrity leading to muscular dystrophy. J Cell Biol 1999; 147:913-20.

5. Scaffidi P, Misteli T. Lamin A-dependent nuclear defects in human aging. Science 2006; 312:1059-63.

6. Goldman RD, Shumaker DK, Erdos MR, Eriksson M, Goldman AE, Gordon LB, et al. Accumulation of mutant lamin A causes progressive changes in nuclear architecture in Hutchinson-Gilford progeria syndrome. Proc Natl Acad Sci U S A 2004; 101:8963-8.

7. Taimen P, Pfleghaar K, Shimi T, Moller D, Ben-Harush K, Erdos MR, et al. A progeria mutation reveals functions for lamin $A$ in nuclear assembly, architecture, and chromosome organization. Proc Natl Acad Sci U S A 2009; 106:20788-93.

8. Maraldi NM, Lattanzi G, Capanni C, Columbaro M, Mattioli E, Sabatelli $P$, et al. Laminopathies: a chromatin affair. Adv Enzyme Regul 2006; 46:33-49.

9. Filesi I, Gullotta F, Lattanzi G, D'Apice MR, Capanni C, Nardone AM, et al. Alterations of nuclear envelope and chromatin organization in mandibuloacral dysplasia, a rare form of laminopathy. Physiol Genomics 2005; 23:150-8.

10. Goldberg M, Harel A, Brandeis M, Rechsteiner T, Richmond TJ, Weiss AM, et al. The tail domain of lamin Dm0 binds histones H2A and H2B. Proc Natl Acad Sci USA 1999; 96:2852-57.

11. de Wit E, van Steensel B. Chromatin domains in higher eukaryotes: insights from genome-wide mapping studies. Chromosoma 2009; 118:25-36.

12. Guelen L, Pagie L, Brasset E, Meuleman W, Faza MB, Talhout W, et al. Domain organization of human chromosomes revealed by mapping of nuclear lamina interactions. Nature 2008; 453:948-51.

13. Pickersgill $H$, Kalverda B, de Wit E, Talhout W, Fornerod $M$, van Steensel B. Characterization of the Drosophila melanogaster genome at the nuclear lamina. Nat Genet 2006; 38:1005-14.

14. Taniura H, Glass C, Gerace L. A Chromatin Binding Site in the Tail Domain of Nuclear Lamins That Interacts with Core Histones. The journal of Cell Biology 1995; 131:33-44.

15. Stierlé V, Couprie J, Östlund C, Krimm I, Zinn-Justin S, Hossenlopp P, et al. The Carboxyl-Terminal Region Common to Lamins $\mathrm{A}$ and $\mathrm{C}$ Contains a DNA Binding Domain. Biochemistry 2003; 42:4819-28.

16. Dechat T, Pfleghaar K, Sengupta K, Shimi T, Shumaker DK, Solimando L, et al. Nuclear lamins: major factors in the structural organization and function of the nucleus and chromatin. Genes Dev 2008; 22:832-53.

17. Pegoraro G, Kubben N, Wickert U, Gohler H, Hoffmann K, Misteli T. Ageing-related chromatin defects through loss of the NURD complex. Nat Cell Biol 2009; 11:1261-7.

18. Rybkin, II, Markham DW, Yan Z, Bassel-Duby R, Williams RS, Olson EN. Conditional expression of SV40 T-antigen in mouse cardiomyocytes facilitates an inducible switch from proliferation to differentiation. J Biol Chem 2003; 278:15927-34. 
19. Romano A, Adriaens M, Kuenen S, Delvoux B, Dunselman G, Evelo C, et al. Identification of novel ER-alpha target genes in breast cancer cells: gene- and cellselective co-regulator recruitment at target promoters determines the response to 17beta-estradiol and tamoxifen. Mol Cell Endocrinol 2010; 314:90-100.

20. Dennis G, Jr., Sherman BT, Hosack DA, Yang J, Gao W, Lane HC, et al. DAVID: Database for Annotation, Visualization, and Integrated Discovery. Genome Biol 2003; 4:P3.

21. Huang da W, Sherman BT, Lempicki RA. Systematic and integrative analysis of large gene lists using DAVID bioinformatics resources. Nat Protoc 2009; 4:44-57.

22. Ji X, Li W, Song J, Wei L, Liu XS. CEAS: cis-regulatory element annotation system. Nucleic Acids Res 2006; 34:W551-4.

23. Cartharius $K$, Frech $K$, Grote $K$, Klocke B, Haltmeier $M$, Klingenhoff $A$, et al. MatInspector and beyond: promoter analysis based on transcription factor binding sites. Bioinformatics 2005; 21:2933-42.

24. Meaburn KJ, Gudla PR, Khan S, Lockett SJ, Misteli T. Disease-specific gene repositioning in breast cancer. J Cell Biol 2009; 187:801-12.

25. Meaburn KJ, Misteli T. Locus-specific and activity-independent gene repositioning during early tumorigenesis. J Cell Biol 2008; 180:39-50.

26. Kubben N, Voncken J, Demmers JA, Calis C, van Almen G, Pinto Y, et al. Identification of differential protein interactors of lamin $A$ and progerin. Nucleus in press., 2010.

27. IBA bioTAGnology. Expression and purification of proteins using Strep-tag and/or 6xHistidine-tag. A comprehensive Manual. 2005.

28. Junttila MR, Saarinen S, Schmidt T, Kast J, Westermarck J. Single-step Strep-tag purification for the isolation and identification of protein complexes from mammalian cells. Proteomics 2005; 5:1199-203.

29. Witte CP, Noel LD, Gielbert J, Parker JE, Romeis T. Rapid one-step protein purification from plant material using the eight-amino acid Strepll epitope. Plant Mol Biol 2004; 55:135-47.

30. Schirmer EC, Guan T, Gerace L. Involvement of the Lamin Rod Domain in Heterotypic Lamin Interactions Important for Nuclear Organization. The Journal of Cell Biology 2001; 153:479-89.

31. Scaffidi P, Misteli T. Lamin A-dependent misregulation of adult stem cells associated with accelerated ageing. Nat Cell Biol 2008; 10:452-9.

32. Shevelyov YY, Lavrov SA, Mikhaylova LM, Nurminsky ID, Kulathinal RJ, Egorova KS, et al. The B-type lamin is required for somatic repression of testis-specific gene clusters. Proc Natl Acad Sci U S A 2009; 106:3282-7.

33. Lehmann J, Retz M, Harder J, Krams M, Kellner U, Hartmann J, et al. Expression of human beta-defensins 1 and 2 in kidneys with chronic bacterial infection. BMC Infect Dis 2002; 2:20.

34. Lomvardas S, Barnea G, Pisapia DJ, Mendelsohn M, Kirkland J, Axel R. Interchromosomal interactions and olfactory receptor choice. Cell 2006; 126:403-13.

35. Aoki K, Meng G, Suzuki K, Takashi T, Kameoka Y, Nakahara K, et al. RP58 associates with condensed chromatin and mediates a sequence-specific transcriptional repression. J Biol Chem 1998; 273:26698-704.

36. Meng G, Inazawa J, Ishida R, Tokura K, Nakahara K, Aoki K, et al. Structural analysis of the gene encoding RP58, a sequence-specific transrepressor associated with heterochromatin. Gene 2000; 242:59-64.

37. Zink D, Amaral MD, Englmann A, Lang S, Clarke LA, Rudolph C, et al. Transcriptiondependent spatial arrangements of CFTR and adjacent genes in human cell nuclei. J Cell Biol 2004; 166:815-25. 
38. Sadoni N, Targosz BS, Englmann A, Fesser S, Koch J, Schindelhauer D, et al. Transcription-dependent spatial arrangements of CFTR and conserved adjacent loci are not conserved in human and murine nuclei. Chromosoma 2008; 117:381-97.

39. Huang da W, Sherman BT, Tan Q, Kir J, Liu D, Bryant D, et al. DAVID Bioinformatics Resources: expanded annotation database and novel algorithms to better extract biology from large gene lists. Nucleic Acids Res 2007; 35:W169-75.

40. Kubben $\mathrm{N}$, Konings $\mathrm{G}$, Voncken J, Houten S, Gijbels $\mathrm{M}$, van Erk A, et al. In preparation: Post-natal myogenic and adipogenic development defects and metabolic impairment upon loss of A-type lamins., 2010.

41. Akhunova A, Arbieva Z, Grove D, Kubista M, Shipley G. Real-Time PCR Tech Guide. A troubleshooting guide: Experts guive their advice on how to conduct real-time PCR. In: Curtin C, ed., 2009. 


\section{Supplemental Figures and Tables}

A
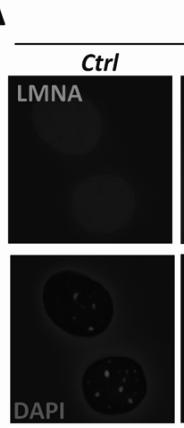

LMNA ${ }^{K O-\%}$ MEF

OST-A
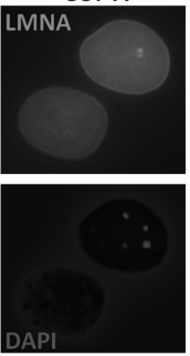

OST-P

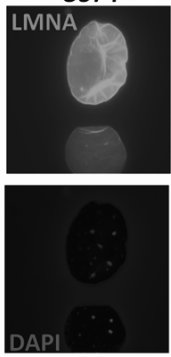

B $\frac{\text { NkITAg }}{\text { CtrI LMNA }}$

ShRNA SHRNA

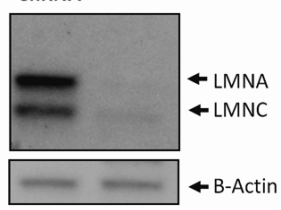

Figure S1. Characteristics of cell lines expressing One-STrEP tagged lamin A and progerin. (A) Immunofluorescent signal of lamin A/C indicating a preferred localization of OST-A and OST-P at the nuclear rim in LMNA ${ }^{\mathrm{KO}-{ }_{-}^{-}}$MEFs. DNA is stained with DAPI. (B) Western blot for Bactin and endogenous lamin $\mathrm{A} / \mathrm{C}$ in NkITAg cells infected with control and LMNA shRNA. 
A

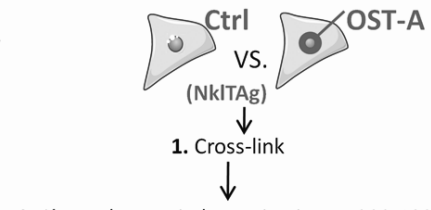

2. Shear chromatin by sonication to $200-1000 \mathrm{bp}$

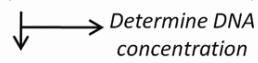

3. Dilute samples to equal concentration of DNA

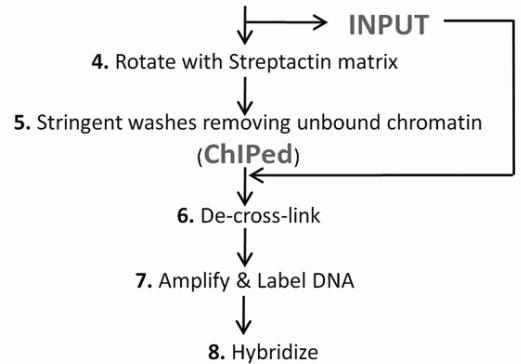

a. ChIPed NkITAg Ctrl|cy-3 / ChIPed NkITAg OSTACy-5

b. ChIPed NkITAg Ctrlly-3 / ChIPed NkITAg OSTACY-5

c. Input NkITAg OSTACy-3 / ChIPed NkITAg OSTACY-5

d. Input NkITAg OSTA ${ }^{\text {Cy-3 }} /$ ChIPed NkITAg OSTA ${ }^{\text {Cy-5 }}$

$\downarrow$

8. In-between array biostatistic analysis

$\downarrow$

9. OST-A interacting gene promoters (identified in $8 a, 8 b, 8 c$ and $8 d$ )
B

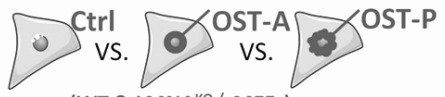

(WT \& LMNA ${ }^{\mathrm{KO}-\%}$ MEFS)

1. Cross-link

$\downarrow$

2. Shear chromatin by sonication to $200-1000 \mathrm{bp}$

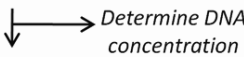

3. Dilute samples to equal concentration of DNA

4. Rotate with Streptactin matrix

$\downarrow$

5. Stringent washes removing unbound chromatin

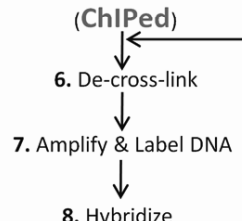

8. Hybridize

a. ChIPed WT MEF Ctrl|cy-3 / ChIPed WT MEF OSTACY-5

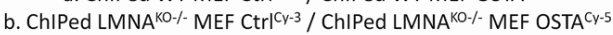

c. ChIPed WT MEF OSTACY-3 / ChIPed WT MEF OST-PCY-5

d. ChIPed LMNA ${ }^{\text {KO }-\%}$ MEF OSTA ${ }^{\text {CY-3 }}$ / ChIPed LMNA ${ }^{\text {KO }-\%}$ MEF OST-PCY-5<smiles>[AlH][V]</smiles>

8. In-between array biostatistic analysis

$\downarrow$

9. OST-A (identified in 8a and 8b) and OST-P (identified in $8 \mathrm{c}$ and 8d) interacting gene promoters

Figure S2. Experimental workflow of lamin A and progerin One-STrEP pulldown. (A) Overview of the experimental procedures applied to determine lamin A interacting genes in NkITAG cells. The chromatin of formaldehyde cross-linked control vector and OST-A expressing NkITAg cells was sheared by sonication to an average length of 200-1000 base pairs. After determining the DNA concentration, samples were further diluted to contain equal concentrations of DNA. Strep-Tactin matrix was added to these input samples for overnight precipitation of OST-A. After removing unbound chromatin by high stringency washes, the pull-down fraction, as well as aliquots of input samples were de-cross-linked, and chromatin was isolated for subsequent amplification and labeling with $\mathrm{Cy}-3$ and $\mathrm{Cy}-5$. Indicated samples were hybridized on genome-wide promoter arrays, and an in-between array biostatistic analysis (See material \& methods) was applied to identify lamin A associated gene promoters. (B) For the identification of lamin $A$ and progerin associated gene promoters a similar technical procedure was applied on OST-A, OST-P and control vectors infected WT and LMNA ${ }^{\mathrm{KO}-\gamma_{-}}$MEFs. OSTA- and OST-P associated gene promoters needed to be recognized as such independently in al arrays in both WT and LMNA ${ }^{\mathrm{KO}-\%} \mathrm{MEFs}$, and are collectively referred to as MEF OST-A and OST-P ChIP targets in this paper. 


\section{Y}

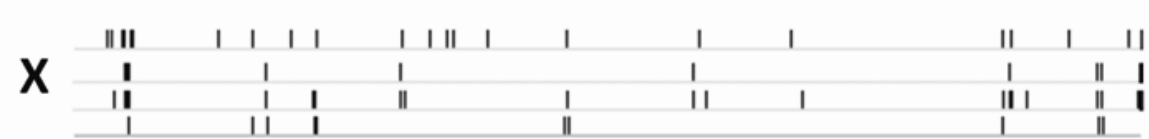

19

\begin{tabular}{|c|c|c|c|c|c|c|c|}
\hline 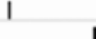 & III I & I & & 11 & 1 & 1 & $\|$ \\
\hline 11 & 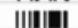 & 1 & i & 1 & & & \\
\hline $1 \|$ & \|\|$\|$ & 1 & 111 & 11 & 1 & 1 & \\
\hline
\end{tabular}

18

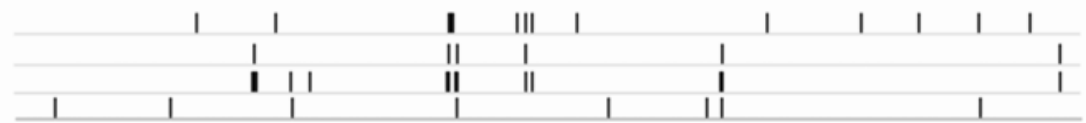

17
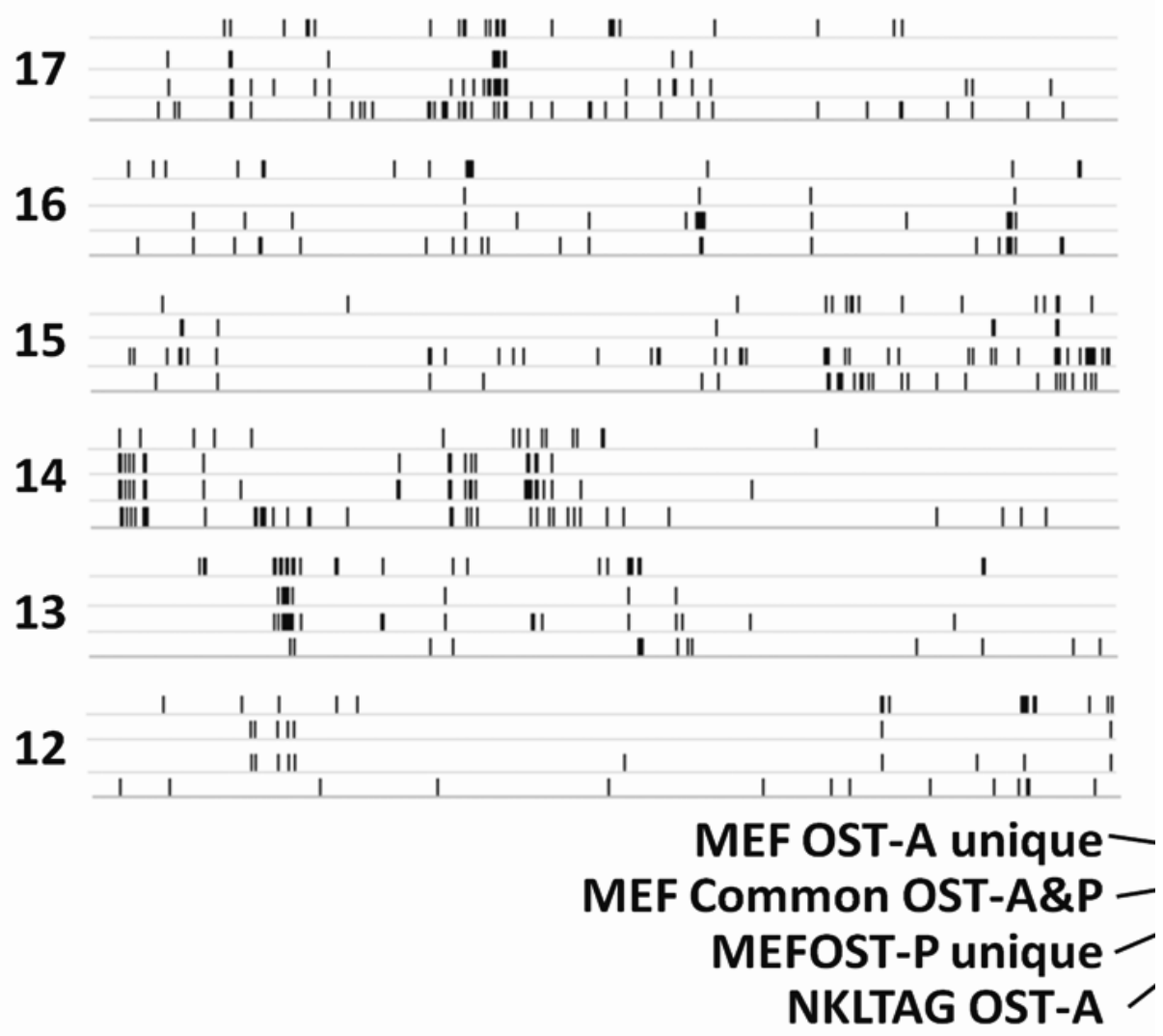

Figure S3. Genomic localization plots of all identified lamin A- and progerin-interacting genes. For each chromosome identified lamin A targets in NkITAg cells (bottom line) and lamin A unique interactors, progerin unique interactors and common interactors in MEFs (upper three lines) are plotted according to their genomic localization. A correction for absolute chromosome size has been applied, creating plots of equal length. 

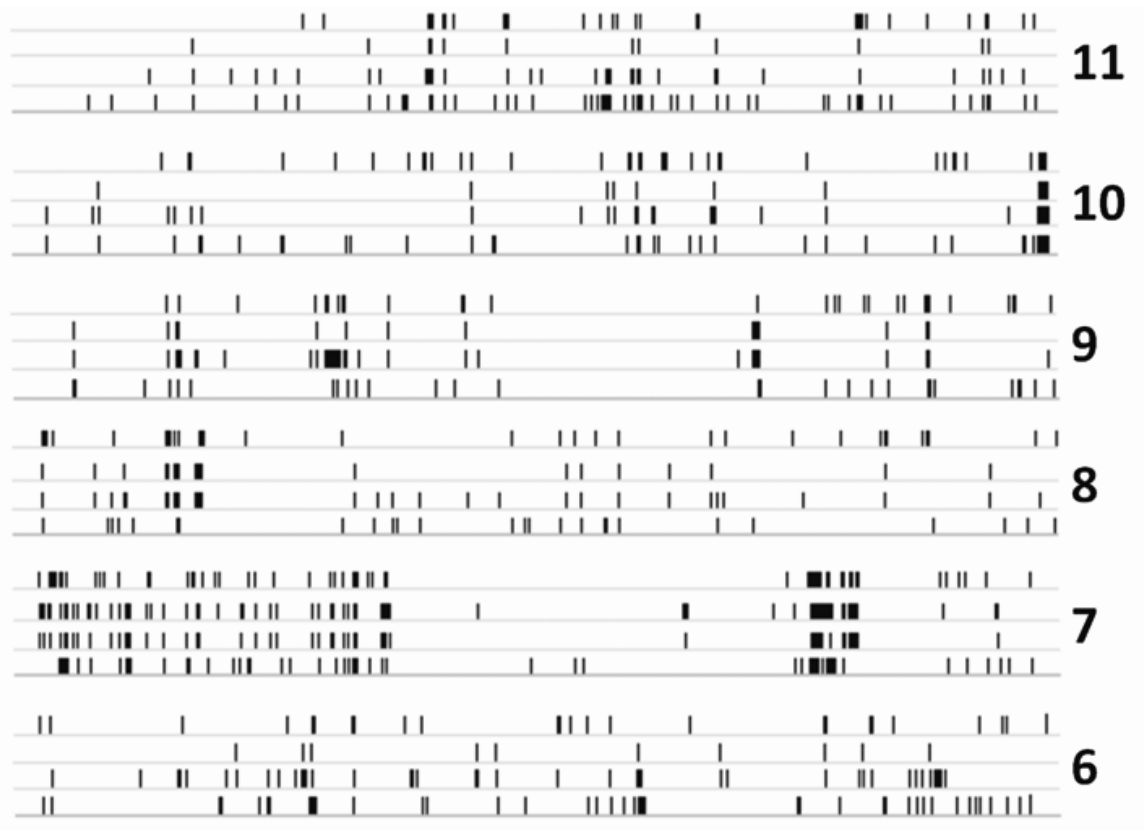

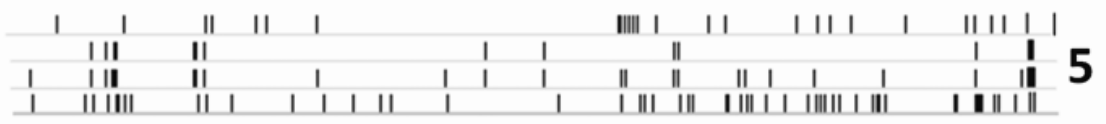
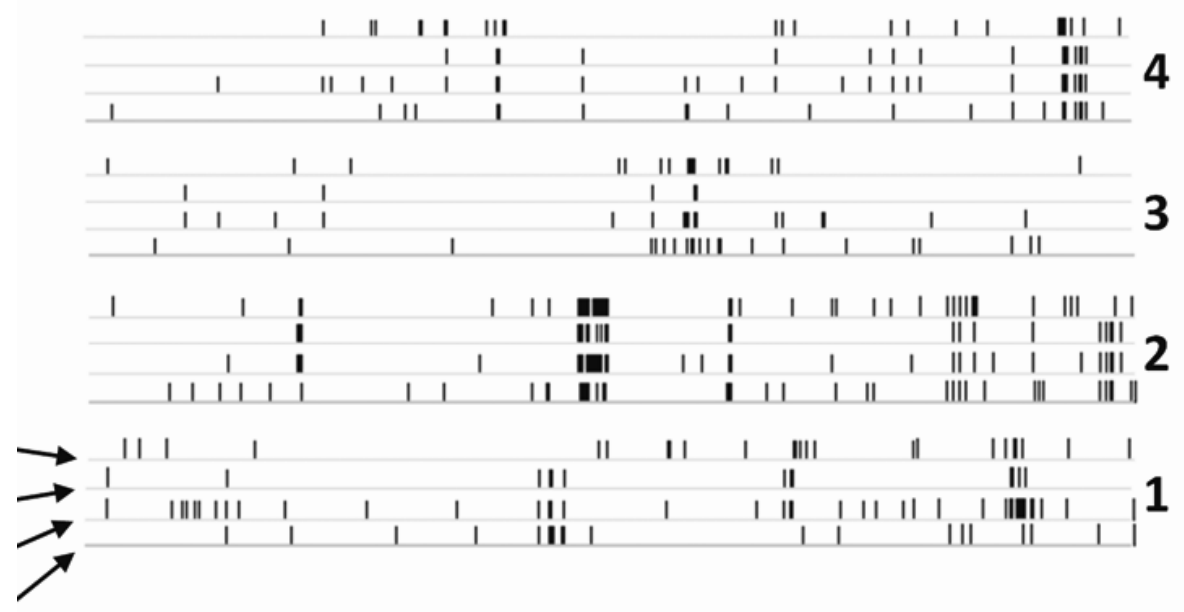

Figure S3 (Continued). 
A

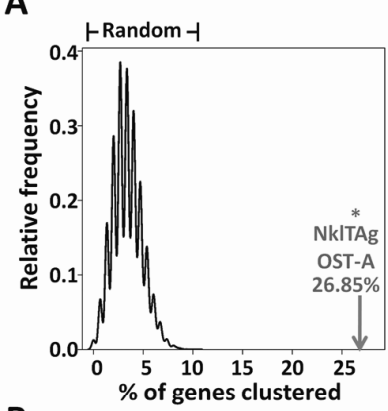

B

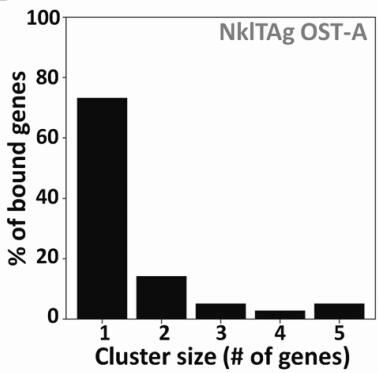

C

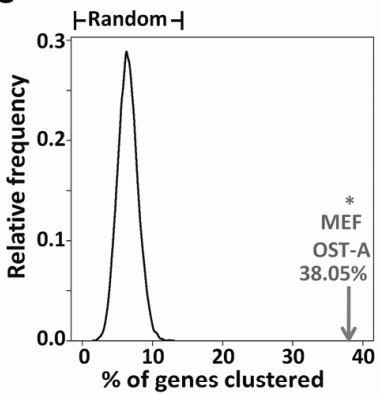

D

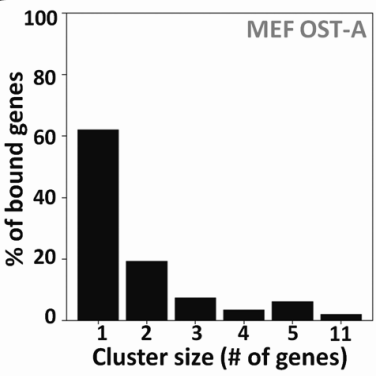

$E$

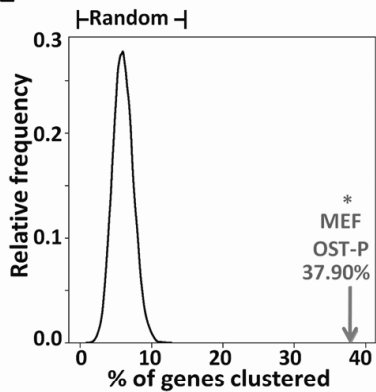

$\mathbf{F}$

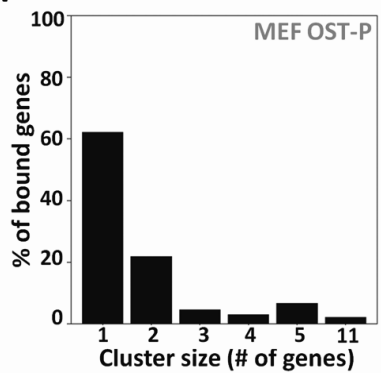

Figure S4. Cluster analysis of lamin A- and progerin-interacting genes. (A) Lamin A-associated genes in NkITAg cells were analyzed for their occurrence in gene clusters, defined as two or more lamin $A$ targets being genomically located adjacent to each other without interruption of a non targets. The percentage of lamin Aassociated targets representated in clusters is indicated, and compared by Fisher's exact test to randomly expected occurrences of gene clusters (asterisk $p<1 \times 10^{-4}$ ). (B) Sizes of identified gene clusters for lamin Aassociated genes in NkITAg are indicated by relative frequency. Targets can be divided in unclustered (cluster size $=1$ gene) and clustered (cluster size $>1$ gene). Comparable analysis was performed for lamin A-targets $(\mathbf{C}, \mathbf{D})$ and progerin-associated genes $(\mathbf{E}, \mathbf{F})$ in MEFs.
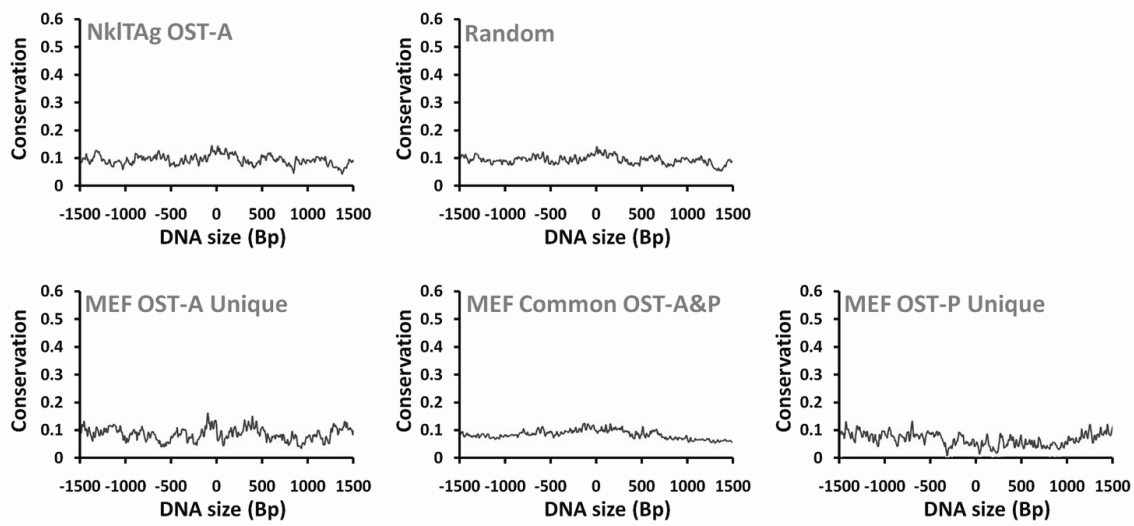

Figure S5. Genomic conservation plots of lamin A and/or progerin interactors. The CEAS database was used to create genomic conservation plots of ChIPed DNA fragments elongated at both ends to a total size of $3 \mathrm{~kb}$, for lamin A unique, progerin unique and common interactors in MEFs as well as lamin A interactors in NkITAg. All conservation levels fluctuated around 0.1 and were comparable to random promoter sequences. 
Table S2. Transcription Factor Motif (TFM) analysis of lamin A- and progerin-associated genes.

\begin{tabular}{|c|c|c|c|c|c|c|c|c|}
\hline \multirow[b]{2}{*}{ TF Family } & \multirow[b]{2}{*}{$P$-value } & \multicolumn{2}{|c|}{ MEF OST-A unique } & \multirow{2}{*}{$\begin{array}{c}\text { MEF OST-A\&P } \\
\text { Common } \\
N\end{array}$} & \multicolumn{2}{|c|}{ MEF OST-P unique } & \multicolumn{2}{|c|}{ NKLTAG OST-A } \\
\hline & & $N$ & $P$-value & & P-value & $N$ & P-value & $N$ \\
\hline V\$BRN5 & $1.0 \mathrm{E}+00$ & 99 & $1.1 \mathrm{E}-11$ & 317 & $3.8 \mathrm{E}-01$ & 97 & $2.4 \mathrm{E}-03$ & 207 \\
\hline V\$SRFF & 7.8E-01 & 92 & $2.8 \mathrm{E}-11$ & 268 & 9.7E-01 & 64 & 3.0E-03 & 170 \\
\hline V\$OCT1 & 9.7E-01 & 148 & $9.8 \mathrm{E}-11$ & 374 & $3.4 \mathrm{E}-01$ & 125 & $5.9 \mathrm{E}-03$ & 254 \\
\hline V\$BPTF & 8.3E-01 & 32 & 2.7E-09 & 126 & 7.5E-02 & 36 & 9.5E-06 & 86 \\
\hline V\$HOXC & $1.0 \mathrm{E}+00$ & 84 & $9.4 \mathrm{E}-09$ & 291 & 8.9E-01 & 81 & 2.1E-02 & 188 \\
\hline V\$RUSH & $9.8 \mathrm{E}-01$ & 106 & 3.9E-08 & 302 & 4.9E-01 & 94 & $8.1 \mathrm{E}-02$ & 193 \\
\hline V\$GFI1 & $1.0 \mathrm{E}+00$ & 46 & $6.3 \mathrm{E}-08$ & 200 & $8.6 \mathrm{E}-01$ & 49 & 5.7E-03 & 128 \\
\hline V\$BRNF & 9.7E-01 & 128 & $9.9 \mathrm{E}-08$ & 336 & $4.2 \mathrm{E}-01$ & 110 & $1.3 \mathrm{E}-03$ & 233 \\
\hline V\$AIRE & $7.4 \mathrm{E}-01$ & 34 & 3.9E-06 & 115 & $6.0 \mathrm{E}-01$ & 28 & $7.8 \mathrm{E}-05$ & 83 \\
\hline V\$STALE & 5.9E-01 & 97 & $5.8 \mathrm{E}-06$ & 249 & 9.7E-01 & 65 & 5.9E-05 & 181 \\
\hline V\$PIT1 & $1.0 \mathrm{E}+00$ & 43 & $1.0 \mathrm{E}-05$ & 172 & $8.1 \mathrm{E}-01$ & 44 & $1.0 \mathrm{E}-01$ & 105 \\
\hline V\$CLOX & $9.5 \mathrm{E}-01$ & 110 & 3.3E-05 & 290 & 5.1E-01 & 94 & 3.5E-02 & 197 \\
\hline V\$TEAF & $8.5 \mathrm{E}-01$ & 62 & 4.1E-05 & 182 & 2.3E-01 & 58 & $2.4 \mathrm{E}-02$ & 120 \\
\hline V\$ZZFTR & $9.4 \mathrm{E}-01$ & 54 & 5.1E-05 & 170 & 7.9E-01 & 45 & 2.9E-04 & 124 \\
\hline V\$EVI1 & 9.7E-01 & 143 & $6.8 \mathrm{E}-05$ & 350 & 7.7E-01 & 116 & 2.6E-01 & 236 \\
\hline V\$GATA & 9.7E-01 & 132 & $1.4 \mathrm{E}-04$ & 331 & $7.2 \mathrm{E}-01$ & 109 & $8.8 \mathrm{E}-02$ & 227 \\
\hline V\$PDX1 & 9.9E-01 & 65 & $2.2 \mathrm{E}-04$ & 206 & $5.6 \mathrm{E}-01$ & 63 & $1.4 \mathrm{E}-03$ & 149 \\
\hline V\$DMRT & 9.9E-01 & 109 & 3.7E-04 & 290 & 7.7E-01 & 92 & $3.8 \mathrm{E}-03$ & 208 \\
\hline V\$LLHXF & 9.9E-01 & 110 & $4.8 \mathrm{E}-04$ & 295 & $9.5 \mathrm{E}-01$ & 89 & $1.6 \mathrm{E}-02$ & 208 \\
\hline O\$PTBP & $1.0 \mathrm{E}+00$ & 40 & 4.9E-04 & 151 & $8.2 \mathrm{E}-01$ & 40 & 9.9E-02 & 97 \\
\hline V\$HBOX & $9.9 \mathrm{E}-01$ & 116 & 5.4E-04 & 306 & 7.1E-01 & 100 & 2.4E-02 & 215 \\
\hline V\$NNEUR & $9.0 \mathrm{E}-01$ & 91 & 7.0E-04 & 240 & $1.0 \mathrm{E}+00$ & 62 & 7.4E-01 & 145 \\
\hline V\$IIRXF & $8.5 \mathrm{E}-01$ & 46 & 7.0E-04 & 138 & 7.0E-01 & 38 & 2.0E-03 & 101 \\
\hline V\$HNF6 & $9.8 \mathrm{E}-01$ & 71 & 7.4E-04 & 208 & 9.0E-01 & 58 & $2.5 \mathrm{E}-02$ & 144 \\
\hline V\$HMTB & 7.6E-01 & 64 & 7.6E-04 & 174 & 7.7E-02 & 62 & $1.2 \mathrm{E}-02$ & 122 \\
\hline V\$CART & $1.0 \mathrm{E}+00$ & 100 & 8.3E-04 & 289 & 7.9E-01 & 92 & 1.7E-01 & 195 \\
\hline V\$FAST & $1.0 \mathrm{E}+00$ & 66 & $9.0 \mathrm{E}-04$ & 207 & $3.5 \mathrm{E}-01$ & 68 & $1.3 \mathrm{E}-02$ & 146 \\
\hline V\$DLXF & $1.0 \mathrm{E}+00$ & 60 & $1.3 \mathrm{E}-03$ & 201 & $8.4 \mathrm{E}-01$ & 58 & 2.6E-01 & 129 \\
\hline V\$SIXF & 9.9E-01 & 48 & $1.5 \mathrm{E}-03$ & 161 & $1.0 \mathrm{E}+00$ & 35 & 1.6E-01 & 104 \\
\hline V\$AP1F & $9.3 \mathrm{E}-01$ & 55 & $1.6 \mathrm{E}-03$ & 163 & 6.0E-01 & 49 & 5.7E-03 & 118 \\
\hline V\$MEF2 & $1.0 \mathrm{E}+00$ & 60 & $1.9 \mathrm{E}-03$ & 219 & 5.0E-01 & 71 & $2.1 \mathrm{E}-01$ & 144 \\
\hline V\$BCL6 & $1.0 \mathrm{E}+00$ & 67 & $2.0 \mathrm{E}-03$ & 205 & $6.8 \mathrm{E}-01$ & 63 & $3.8 \mathrm{E}-01$ & 130 \\
\hline V\$PCBE & 3.6E-01 & 43 & $2.2 \mathrm{E}-03$ & 109 & $9.2 \mathrm{E}-01$ & 25 & 3.1E-01 & 65 \\
\hline V\$SKKHD & $1.0 \mathrm{E}+00$ & 135 & $2.3 \mathrm{E}-03$ & 346 & $2.0 \mathrm{E}-01$ & 125 & $8.0 \mathrm{E}-03$ & 250 \\
\hline V\$BTBF & 6.0E-01 & 30 & 2.9E-03 & 86 & 9.9E-01 & 15 & $6.9 \mathrm{E}-03$ & 63 \\
\hline O\$YTBP & 9.9E-01 & 19 & 2.9E-03 & 86 & $7.8 \mathrm{E}-01$ & 21 & 5.9E-02 & 57 \\
\hline V\$SF1F & $9.2 \mathrm{E}-01$ & 58 & $3.1 \mathrm{E}-03$ & 166 & $9.8 \mathrm{E}-01$ & 40 & 1.3E-01 & 110 \\
\hline V\$PARF & $9.8 \mathrm{E}-01$ & 118 & 3.3E-03 & 298 & 7.0E-01 & 99 & $5.3 \mathrm{E}-02$ & 210 \\
\hline V\$HOXF & $1.0 \mathrm{E}+00$ & 132 & $4.0 \mathrm{E}-03$ & 333 & 4.4E-01 & 117 & $2.9 \mathrm{E}-03$ & 244 \\
\hline V\$PBXC & $5.4 \mathrm{E}-01$ & 86 & $4.2 \mathrm{E}-03$ & 207 & $9.9 \mathrm{E}-01$ & 53 & $1.5 \mathrm{E}-02$ & 149 \\
\hline V\$\$LEFF & 9.9E-01 & 77 & $4.5 \mathrm{E}-03$ & 223 & 9.7E-01 & 62 & $1.3 \mathrm{E}-01$ & 152 \\
\hline V\$OVOL & $9.9 \mathrm{E}-01$ & 63 & $4.8 \mathrm{E}-03$ & 191 & 8.7E-01 & 55 & $1.3 \mathrm{E}-01$ & 129 \\
\hline V\$SPAR & $9.9 \mathrm{E}-01$ & 19 & $5.4 \mathrm{E}-03$ & 83 & 8.7E-01 & 19 & $2.2 \mathrm{E}-01$ & 51 \\
\hline V\$NBRE & $1.0 \mathrm{E}+00$ & 25 & $7.8 \mathrm{E}-03$ & 111 & 8.3E-01 & 29 & 7.7E-03 & 83 \\
\hline O\$INRE & 8.9E-01 & 65 & 9.5E-03 & 175 & 9.9E-01 & 44 & 1.0E-04 & 141 \\
\hline V\$RP58 & 6.0E-01 & 37 & $9.6 \mathrm{E}-03$ & 99 & 2.7E-01 & 33 & 4.7E-03 & 76 \\
\hline V\$NKXH & $1.0 \mathrm{E}+00$ & 129 & $1.2 \mathrm{E}-02$ & 327 & $5.8 \mathrm{E}-01$ & 114 & $1.5 \mathrm{E}-01$ & 230 \\
\hline V\$NF1F & $9.8 \mathrm{E}-01$ & 62 & $1.2 \mathrm{E}-02$ & 181 & $9.6 \mathrm{E}-01$ & 49 & 4.4E-01 & 116 \\
\hline V\$ABDB & $1.0 \mathrm{E}+00$ & 131 & $1.3 \mathrm{E}-02$ & 328 & $6.2 \mathrm{E}-01$ & 114 & 2.3E-01 & 229 \\
\hline V\$PERO & $9.3 \mathrm{E}-01$ & 87 & $1.4 \mathrm{E}-02$ & 224 & $9.8 \mathrm{E}-01$ & 63 & $2.8 \mathrm{E}-01$ & 151 \\
\hline O\$VTBP & 9.9E-01 & 127 & 1.7E-02 & 313 & 8.6E-01 & 104 & 1.4E-02 & 229 \\
\hline V\$GREF & 9.9E-01 & 105 & 1.7E-02 & 272 & $1.0 \mathrm{E}+00$ & 76 & 2.7E-01 & 187 \\
\hline
\end{tabular}




\begin{tabular}{|c|c|c|c|c|c|c|c|c|}
\hline V\$NKX6 & $1.0 \mathrm{E}+00$ & 73 & 1.7E-02 & 230 & $6.9 \mathrm{E}-01$ & 75 & 4.7E-03 & 173 \\
\hline V\$CHRF & $1.0 \mathrm{E}+00$ & 39 & $1.9 \mathrm{E}-02$ & 154 & $9.5 \mathrm{E}-01$ & 41 & $3.3 \mathrm{E}-02$ & 112 \\
\hline V\$CDXF & $1.0 \mathrm{E}+00$ & 86 & $2.2 \mathrm{E}-02$ & 237 & $8.0 \mathrm{E}-01$ & 76 & $3.1 \mathrm{E}-01$ & 161 \\
\hline V\$THAP & $9.0 \mathrm{E}-01$ & 22 & $2.2 \mathrm{E}-02$ & 73 & $9.3 \mathrm{E}-01$ & 16 & $3.9 \mathrm{E}-01$ & 44 \\
\hline V\$AARF & $1.0 \mathrm{E}+00$ & 6 & $2.5 \mathrm{E}-02$ & 52 & $6.1 \mathrm{E}-02$ & 21 & $6.5 \mathrm{E}-01$ & 27 \\
\hline V\$HAND & $1.0 \mathrm{E}+00$ & 134 & 4.4E-02 & 330 & $1.0 \mathrm{E}+00$ & 95 & $7.8 \mathrm{E}-01$ & 223 \\
\hline V\$NKX1 & $1.0 \mathrm{E}+00$ & 30 & $7.1 \mathrm{E}-02$ & 112 & $9.6 \mathrm{E}-01$ & 28 & $7.8 \mathrm{E}-01$ & 66 \\
\hline$\ldots$. & $\ldots$. & $\ldots$ & $\ldots$. & $\ldots$ & $\ldots$. & $\ldots$. & $\ldots$ & $\ldots$ \\
\hline V\$̦ZF5F & $1.0 \mathrm{E}+00$ & 14 & $1.0 \mathrm{E}+00$ & 11 & $1.0 \mathrm{E}+00$ & 5 & $1.0 \mathrm{E}+00$ & 15 \\
\hline
\end{tabular}

Results of the TFM analysis using MatInspector software and the Genomatix transcription factor motif database (www.genomatix.de) show the amount of TFM (N) identified in ChIPed DNA fragments and corresponding significance levels for 170 transcription factor families. Significant results are indicated in bold $\left(p<0.05\right.$; Statistical analysis described previously $\left.{ }^{23}\right)$. Extra note: This list was shortened for publication purposes.

Table S3. Absolute and relative subnuclear positioning of common and unique lamin A/progerinassociated genes

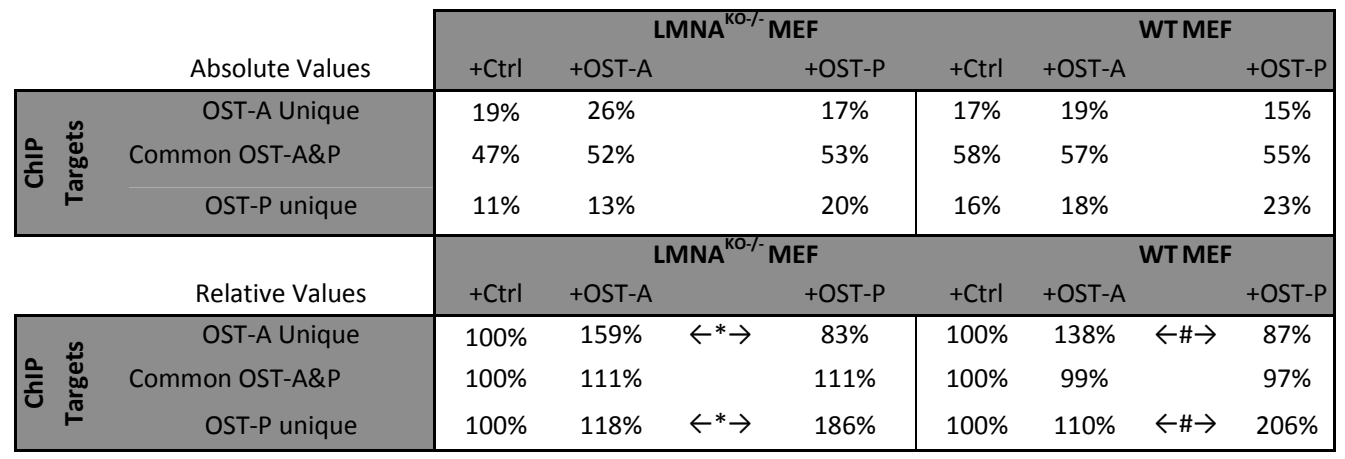

Legend: FISH quantifications (Figure 5B) of OST-Aunique (Eef1a2, Kcnip2, Scn5a, Sdro), common OSTA\&P (Cyp3a25, OLFR804, ABPD, Defcr5) and OST-Punique targets (Daf2, Tcf21, Trim30, Abcb10) were averaged per subclass, to obtain absolute frequencies on lamin B signal overlap. Relative values were calculated by setting the frequency for each individual locus in empty virus infected cells to $100 \%$ and adjusting quantification results in the other cell lines accordingly. Asterisks and hashed indicate respectively significant $(p<0.05$, Student's t-test) and bordeline significant $(0.05<p<0.10$, Student's ttest) differences in lamin B overlap between OST-A and OST-P expressing cells. 
Table S4. Overview of lamin A-associated genes in NkITAg myocytes that change expression levels.

\begin{tabular}{|c|c|c|c|c|c|c|}
\hline $\begin{array}{l}\text { Entrez Gene } \\
\text { ID }\end{array}$ & $\begin{array}{c}\text { Gene } \\
\text { Abbreviatio } \\
\mathrm{n}\end{array}$ & Target Type & $\begin{array}{c}\text { Expression } \\
\text { WT }\end{array}$ & $\begin{array}{l}\text { Expression } \\
\text { LMNA }^{\text {GT- }-}\end{array}$ & $\begin{array}{c}\text { FC } \\
\text { LMNA }^{\text {GT-/- }} \\
\text { vs. WT }\end{array}$ & P-value \\
\hline 13195 & Ddc & NkITAg OST- & 603.2 & 868.3 & 1.44 & 0.01 \\
\hline 258627 & Olfr1504 & NkITAg OST- & 13.3 & 15.5 & 1.17 & 0.01 \\
\hline 53318 & Pdlim3 & NkITAg OST- & 23.0 & 26.3 & 1.14 & 0.01 \\
\hline 13602 & Sparcl1 & NkITAg OST- & 3464.8 & 3939.0 & 1.14 & 0.05 \\
\hline 93674 & $\mathrm{Cml3}$ & NkITAg OST- & 6.1 & 6.3 & 1.03 & 0.04 \\
\hline 68172 & Rpl39l & NkITAg OST- & 8.0 & 8.1 & 1.02 & 0.02 \\
\hline 56640 & Klk4 & NkITAg OST- & 5.5 & 5.6 & 1.02 & 0.05 \\
\hline 239368 & BC030476 & NkITAg OST- & 6.9 & 7.0 & 1.02 & 0.04 \\
\hline 56448 & Cyp2d22 & NkITAg OST- & 10.1 & 8.9 & 0.89 & 0.02 \\
\hline 70101 & Сур4f16 & NkITAg OST- & 15.3 & 11.1 & 0.72 & 0.04 \\
\hline
\end{tabular}

Genes that were identified by ChIP technology to interact with OST-A in NkITAg cardiac myocytes and significantly ( $p<0.05$, no fold-change criterion used; Student's T-test) changed their expression level upon loss of lamin A/C in cardiac tissue (WT vs. LMNAGT-/-). Entrez gene ID, absolute linear expression values, expression fold changes and $p$-values are indicated.

Table S5. Overview of common and differential lamin A/progerin-associated genes in MEFs that change expression levels.

\begin{tabular}{|c|c|c|c|c|c|c|c|c|c|c|c|}
\hline $\begin{array}{l}\text { Entrez } \\
\text { Gene ID }\end{array}$ & $\begin{array}{c}\text { Gene } \\
\text { Abbreviation }\end{array}$ & Target Type & $\begin{array}{c}\text { Expression } \\
W T+C t r l\end{array}$ & $\begin{array}{l}\text { Expression } \\
\text { WT+OST-A }\end{array}$ & $\begin{array}{l}\text { Expression } \\
\text { WT+OST-P }\end{array}$ & $\mathrm{FC} \mathrm{A} / \mathrm{Ctrl}$ & $\begin{array}{c}\mathrm{FC} \\
\mathrm{P} / \mathrm{Ctrl}\end{array}$ & $\begin{array}{l}\mathrm{FC} \\
\mathrm{P} / \mathrm{A}\end{array}$ & $\begin{array}{l}\text { Pvalue } \\
\text { A/Ctrl }\end{array}$ & $\begin{array}{l}\text { Pvalue } \\
\text { P/Ctrl }\end{array}$ & $\begin{array}{c}\text { Pvalue } \\
\text { P/A }\end{array}$ \\
\hline 56229 & Thsd1 & OST-A & 28.1 & 26.0 & 25.5 & 0.92 & 0.91 & 0.98 & 0.05 & 0.48 & 0.89 \\
\hline 245308 & Zdhhc19 & OST-A & 14.4 & 13.5 & 13.8 & 0.94 & 0.96 & 1.02 & 0.11 & 0.02 & 0.49 \\
\hline 244653 & Hydin & OST-A & 9.5 & 9.2 & 9.1 & 0.98 & 0.96 & 0.98 & 0.16 & 0.02 & 0.26 \\
\hline 14128 & Fcer2a & OST-A & 9.2 & 8.9 & 9.0 & 0.96 & 0.97 & 1.01 & 0.03 & 0.02 & 0.14 \\
\hline 14939 & Gzmb & OST-A & 12.8 & 13.8 & 12.8 & 1.08 & 1.00 & 0.92 & 0.01 & 0.88 & 0.06 \\
\hline 70073 & Zdhhc25 & OST-A & 7.7 & 8.0 & 7.9 & 1.04 & 1.03 & 0.99 & 0.03 & 0.06 & 0.60 \\
\hline 209268 & Igsf1 & OST-A & 10.6 & 11.6 & 11.0 & 1.10 & 1.04 & 0.95 & 0.50 & 0.05 & 0.68 \\
\hline 258426 & Olfr995 & OST-A & 5.1 & 5.5 & 5.5 & 1.08 & 1.07 & 0.99 & 0.32 & 0.04 & 0.93 \\
\hline 15564 & $\mathrm{Htr} 5 \mathrm{~b}$ & OST-A & 10.4 & 11.3 & 11.3 & 1.09 & 1.08 & 0.99 & 0.26 & 0.03 & 0.91 \\
\hline 13532 & Dub2 & OST-A & 14.3 & 14.9 & 15.6 & 1.04 & 1.09 & 1.05 & 0.27 & 0.00 & 0.22 \\
\hline 20703 & Serpina1d & OST-A & 8.5 & 7.4 & 9.4 & 0.87 & 1.11 & 1.28 & 0.03 & 0.02 & 0.01 \\
\hline 76071 & Jakmip1 & OST-A & 17.7 & 16.1 & 19.7 & 0.91 & 1.12 & 1.22 & 0.00 & 0.10 & 0.03 \\
\hline 22639 & $\mathrm{Zfa}$ & OST-A & 5.9 & 6.4 & 6.9 & 1.09 & 1.18 & 1.08 & 0.10 & 0.01 & 0.09 \\
\hline 68119 & Cmtm3 & OST-A & 622.9 & 639.2 & 738.3 & 1.03 & 1.19 & 1.15 & 0.82 & 0.01 & 0.29 \\
\hline 213027 & B130050I23Rik & OST-A & 76.4 & 85.3 & 92.1 & 1.12 & 1.20 & 1.08 & 0.08 & 0.03 & 0.24 \\
\hline 625599 & LOC625599 & OST-A & 9.4 & 10.4 & 11.5 & 1.11 & 1.23 & 1.11 & 0.08 & 0.00 & 0.10 \\
\hline 50774 & Krtap5-1 & OST-A & 60.3 & 60.7 & 76.6 & 1.01 & 1.27 & 1.26 & 0.90 & 0.04 & 0.05 \\
\hline 627585 & LOC627585 & Common & 160.2 & 90.1 & 107.5 & 0.56 & 0.67 & 1.19 & 0.00 & 0.02 & 0.08 \\
\hline 381236 & Al747699 & Common & 16.4 & 13.2 & 11.9 & 0.81 & 0.72 & 0.90 & 0.00 & 0.04 & 0.25 \\
\hline 233164 & 4930549006 & Common & 16.6 & 22.5 & 12.2 & 1.36 & 0.73 & 0.54 & 0.24 & 0.01 & 0.08 \\
\hline 258016 & Olfr453 & Common & 11.6 & 10.3 & 9.8 & 0.89 & 0.84 & 0.94 & 0.26 & 0.05 & 0.46 \\
\hline 252829 & Obox5 & Common & 44.3 & 42.4 & 38.1 & 0.96 & 0.86 & 0.90 & 0.63 & 0.03 & 0.32 \\
\hline 258281 & Olfr780 & Common & 5.7 & 5.3 & 5.0 & 0.93 & 0.87 & 0.93 & 0.42 & 0.04 & 0.38 \\
\hline 233099 & Abpb & Common & 7.4 & 7.6 & 6.5 & 1.02 & 0.87 & 0.85 & 0.27 & 0.04 & 0.04 \\
\hline 259070 & Olfr829 & Common & 7.2 & 7.0 & 6.6 & 0.98 & 0.92 & 0.94 & 0.74 & 0.00 & 0.33 \\
\hline 16769 & Dsg4 & Common & 10.1 & 9.9 & 9.4 & 0.98 & 0.93 & 0.95 & 0.37 & 0.01 & 0.08 \\
\hline 258339 & Olfr1269 & Common & 6.5 & 6.3 & 6.0 & 0.97 & 0.93 & 0.96 & 0.47 & 0.02 & 0.38 \\
\hline
\end{tabular}


118 | Chapter 4

\begin{tabular}{|c|c|c|c|c|c|c|c|c|c|c|c|}
\hline 72925 & 39873 & Common & 10.3 & 9.6 & 9.7 & 0.93 & 0.94 & 1.01 & 0.01 & 0.02 & 0.33 \\
\hline 229214 & Gpr103 & Common & 13.4 & 12.9 & 12.6 & 0.97 & 0.94 & 0.97 & 0.00 & 0.56 & 0.78 \\
\hline 110187 & Abpg & Common & 5.5 & 5.1 & 5.2 & 0.92 & 0.94 & 1.02 & 0.00 & 0.01 & 0.03 \\
\hline 387131 & Ssxb9 & Common & 4.1 & 3.6 & 3.9 & 0.88 & 0.95 & 1.09 & 0.00 & 0.14 & 0.05 \\
\hline 258387 & Olfr720 & Common & 7.6 & 7.9 & 7.3 & 1.04 & 0.96 & 0.93 & 0.27 & 0.04 & 0.10 \\
\hline 259119 & Olfr578 & Common & 5.9 & 6.6 & 5.8 & 1.12 & 0.98 & 0.88 & 0.02 & 0.44 & 0.03 \\
\hline 20684 & Sp100 & Common & 17.6 & 16.1 & 17.7 & 0.91 & 1.01 & 1.10 & 0.04 & 0.79 & 0.05 \\
\hline 258464 & Olfr1384 & Common & 6.5 & 7.4 & 6.7 & 1.14 & 1.03 & 0.91 & 0.05 & 0.34 & 0.11 \\
\hline 258771 & Olfr473 & Common & 4.7 & 4.6 & 4.9 & 0.99 & 1.04 & 1.05 & 0.70 & 0.02 & 0.16 \\
\hline 258405 & Olfr1420 & Common & 10.7 & 11.4 & 11.2 & 1.06 & 1.04 & 0.98 & 0.53 & 0.04 & 0.83 \\
\hline 545047 & MGC118309 & Common & 5.7 & 6.0 & 5.9 & 1.06 & 1.05 & 0.99 & 0.24 & 0.01 & 0.80 \\
\hline 259058 & Olfr646 & Common & 9.1 & 10.3 & 9.6 & 1.13 & 1.06 & 0.94 & 0.04 & 0.28 & 0.29 \\
\hline 244187 & Olfr684 & Common & 6.6 & 7.5 & 7.0 & 1.13 & 1.06 & 0.94 & 0.01 & 0.06 & 0.03 \\
\hline 258970 & Olfr1242 & Common & 6.8 & 7.2 & 7.4 & 1.07 & 1.09 & 1.03 & 0.32 & 0.03 & 0.66 \\
\hline 258252 & Olfr813 & Common & 4.9 & 5.5 & 5.4 & 1.12 & 1.11 & 0.99 & 0.01 & 0.05 & 0.71 \\
\hline 27058 & Srp9 & OST-P & 99.1 & 81.9 & 73.4 & 0.83 & 0.74 & 0.90 & 0.03 & 0.00 & 0.05 \\
\hline 110115 & Cyp11b1 & OST-P & 23.7 & 22.6 & 18.9 & 0.96 & 0.80 & 0.83 & 0.55 & 0.05 & 0.08 \\
\hline 109663 & Hoxc11 & OST-P & 9.4 & 9.0 & 8.2 & 0.96 & 0.87 & 0.91 & 0.04 & 0.00 & 0.01 \\
\hline 75553 & 2700069A02Rik & OST-P & 427.7 & 362.9 & 387.7 & 0.85 & 0.91 & 1.07 & 0.01 & 0.00 & 0.05 \\
\hline 66648 & 5730494M16Rik & OST-P & 219.9 & 208.0 & 209.5 & 0.95 & 0.95 & 1.01 & 0.04 & 0.25 & 0.83 \\
\hline 69774 & Ms4a6b & OST-P & 6.6 & 7.2 & 6.5 & 1.08 & 0.98 & 0.91 & 0.04 & 0.30 & 0.03 \\
\hline 58861 & Cysltr1 & OST-P & 8.8 & 8.3 & 8.7 & 0.95 & 0.99 & 1.05 & 0.03 & 0.03 & 0.03 \\
\hline 20014 & Rpn2 & OST-P & 1107.9 & 1123.5 & 1141.0 & 1.01 & 1.03 & 1.02 & 0.53 & 0.04 & 0.48 \\
\hline 67484 & 2310005P05Rik & OST-P & 12.9 & 13.6 & 13.4 & 1.05 & 1.04 & 0.99 & 0.05 & 0.06 & 0.04 \\
\hline 66853 & Pnpla2 & OST-P & 81.5 & 95.0 & 93.4 & 1.17 & 1.15 & 0.98 & 0.01 & 0.10 & 0.75 \\
\hline 75284 & 4930556P03Rik & OST-P & 24.4 & 27.7 & 29.6 & 1.13 & 1.21 & 1.07 & 0.02 & 0.14 & 0.50 \\
\hline 20128 & Trim30 & OST-P & 19.2 & 22.2 & 31.3 & 1.16 & 1.63 & 1.41 & 0.16 & 0.02 & 0.06 \\
\hline
\end{tabular}

Note: Genes that were identified by ChIP technology to interact in MEFs with OST-A only, OST-P only or with both lamins and in addition significantly ( $p<0.05$, no fold-change criterion; Anova) changed their expression level either upon introduction of OST-A or OST-P in WT MEFs. Entrez gene ID, absolute linear expression values, fold changes and significance values between WT MEFs infected with empty virus (Ctrl), OST-A or OST-P are indicated. 


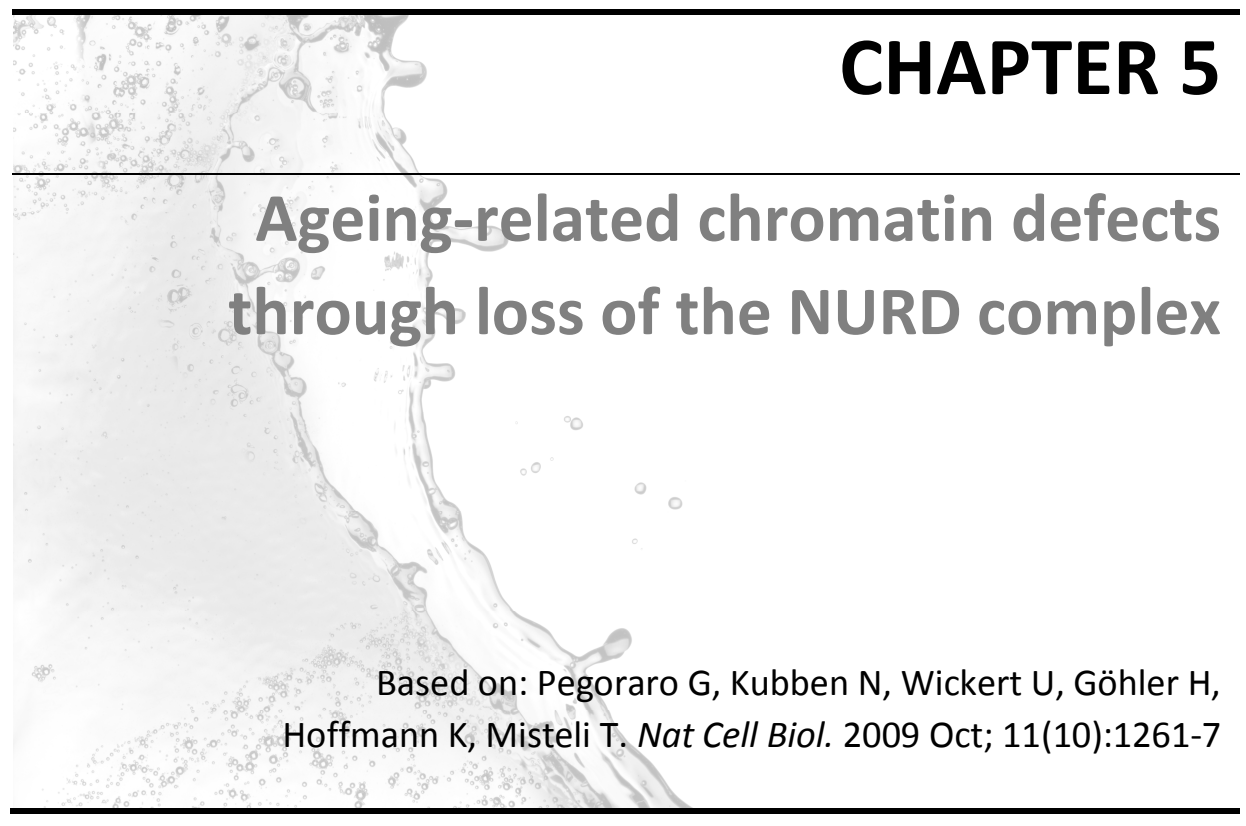




\begin{abstract}
Physiological and premature aging are characterized by multiple defects in chromatin structure and accumulation of persistent DNA damage. Here we identify the NURD remodeling complex as a key modulator of these agingassociated chromatin defects. We demonstrate loss of several NURD components during premature and normal aging and we find agingassociated reduction of HDAC1 activity. Silencing of individual NURD subunits recapitulates some chromatin defects associated with aging and we provide evidence that structural chromatin defects precede DNA damage accumulation. These results outline a molecular mechanism for chromatin defects during aging.
\end{abstract}


A hallmark of normal and premature aging are global changes in chromatin including loss of heterochromatin structure, altered patterns of histone modifications ${ }^{1-4}$, loss of key heterochromatin proteins ${ }^{2-3}$, and increased levels of persistent DNA damage. ${ }^{5-6}$ To begin to elucidate the molecular mechanisms leading to these aging-related chromatin defects, we have taken advantage of the premature aging disorder Hutchinson-Gilford Progeria Syndrome (HGPS). This early childhood disease is caused by a recurrent de-novo point mutation in exon 11 of the lamin $A / C$ gene (LMNA), which encodes for the intermediate filament proteins lamin $A$ and lamin $C$, two of the key architectural proteins of the cell nucleus. ${ }^{7}$ The diseasecausing mutation in $L M N A$ leads to the production of a mutant form of lamin A, referred to as progerin, which lacks 50 aa near the $\mathrm{C}$-terminus, and acts in a dominant negative fashion causing multiple cellular defects of physiological and accelerated-aging. ${ }^{1-2,}{ }^{8-9}$ In particular, HGPS cells exhibit several chromatin defects which are also characteristic for physiological aging including loss of heterochromatin structure, loss of methylation at $\mathrm{H} 3 \mathrm{~K} 9$ and H3K27, down-regulation of the heterochromatin protein HP1, and increased transcription of pericentromeric Satellite III repeats (SatIII). ${ }^{2,4}$ In addition, as normally aged cells, HGPS patient cells exhibit increased steadystate levels of DNA damage.,

To identify the molecular basis of aging-associated chromatin defects, we performed a yeast two hybrid screen using a C-terminal region of lamin $\mathrm{A}$ (aa 562-664) which overlaps with the region deleted in progerin (Fig. 1A). We identified the WD40 domain chromatin protein RBBP4 as a lamin A interactor in four independent experiments. None of the other lamin $A$ fragments tested (aa 1-118, aa 416-568, aa 604-657) interacted with RBBP4 by two-hybrid analysis (data not shown). The interaction between RBBP4 and the aa 562-664 fragment of lamin A was confirmed by GST-pull down (Supplementary Fig. 1A). RBBP4 and RBBP7 are evolutionary conserved histone binding proteins. ${ }^{10}$ They are shared subunits of several multi-protein complexes involved in the establishment of heterochromatin including the NUcleosome Remodeling and Deacetylase (NURD) complex ${ }^{11}$ and the Polycomb PRC2 complex. ${ }^{12}$ RBBP4 is also the p48 subunit of the CAF-1 complex, which assembles chromatin upon DNA replication and DNA damage repair. ${ }^{13}$ In support of a physical interaction between lamin $A$ and $\mathrm{RBBP} 4 / 7$, recombinant forms of both proteins bound in vitro to GST-lamin A 


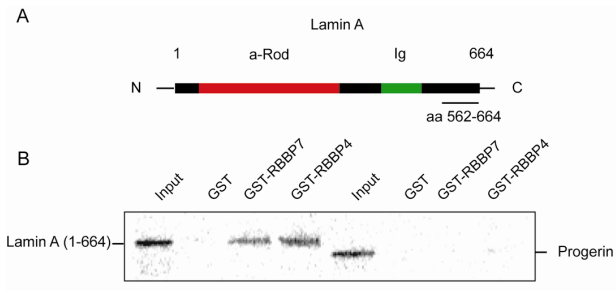

C

D

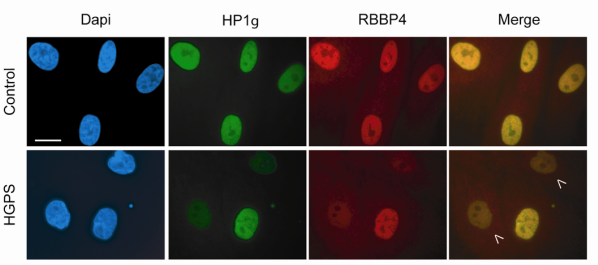

$\mathrm{F}$

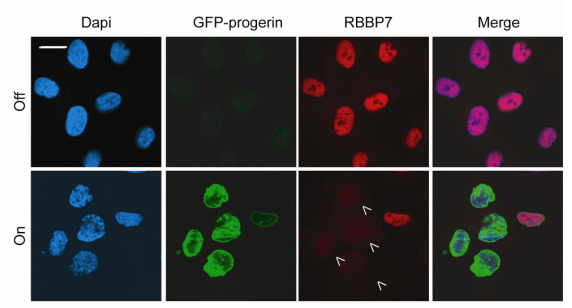

Dapi

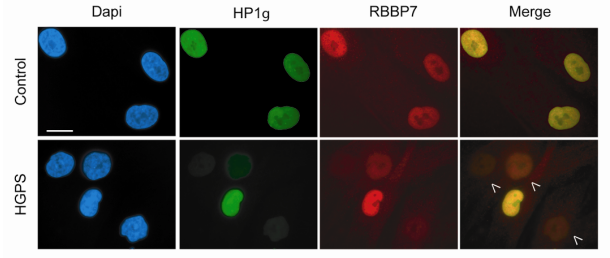

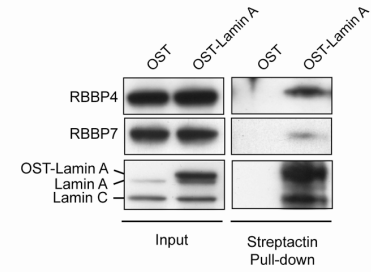
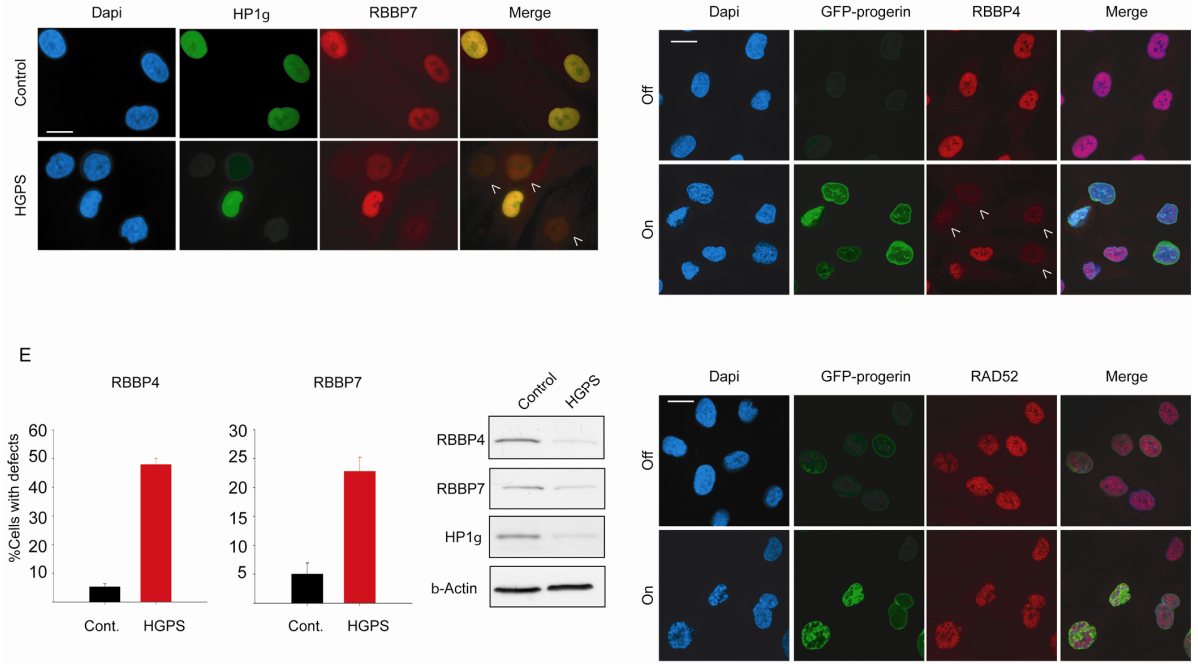

Fig. 1. Loss of RBBP4 and RBBP7 in progerin expressing cells. (A) Schematic representation of lamin A. The fragment used as a bait in the $\mathrm{Y} 2 \mathrm{H}$ assay (aa 562-664) is underlined. (B) GST pulldown assays with recombinant GST-RBBP4/7 and in vitro transcribed and translated lamin A (aa 1-664) or progerin. (C) Crosslinked protein lysates were prepared from U2OS cells expressing One-Strep (OST)-tagged lamin A and then incubated with Streptactin beads. Input and pulled down material was probed by Western blot with antibodies against the indicated endogenous proteins. (D) Immunofluorescence staining on control and HGPS primary dermal fibroblasts with the indicated antibodies. DAPI, 4', 6'-diamidino-2-phenylindole. Quantitative analysis of fluorescence intensity signal in control and HGPS patient cells. The percentages of cells with reduced protein level are indicated. $\mathrm{N}>200$; values represent averages \pm S.D from at least three experiments. Cells were analyzed around population doubling (PD) 25 in the RBBP4 experiments and around PD 20 in the RBBP7 experiments. Control cells were passage-matched to HGPS cells in each experiment. (E) Western blot of cell lysates prepared from control and HGPS dermal fibroblasts. (F) Immunofluorescence with indicated antibodies in wt fibroblasts that were either induced (on) or not induced (off) to express a GFP-tagged version of progerin. Arrowheads indicate cells with reduced levels of heterochromatin proteins. Scale bars: $10 \mu \mathrm{m}$. 
(1-664) and to a lesser extend to a C-terminal fragment (residues 390 - 646) (Fig. 1B; Supplementary Fig. 1B). Importantly, neither protein bound to GSTprogerin which lacks residues 607-657 of mature lamin A (Fig. 1B). The in vivo interaction of RBBP4, and more weakly of RBBP7, with lamin A was confirmed by immunoprecipitation of endogenous RBBP4/7 by overexpressed One-Strep (OST)-tagged lamin A (Fig. 1C). These results identify the two histone chaperone proteins RBBP4 and 7 as lamin A interactors.

To ask whether RBBP4 and RBBP7 are involved in aging-associated chromatin defects, we probed their status in HGPS cells. Strikingly, a substantial fraction $(48+/-2 \%)$ of HGPS patient cells showed reduced protein levels of RBBP4 when compared to passage- and age-matched controls from healthy donors by quantitative immunofluorescence microscopy as previously described (Fig. 1D, E).$^{2-3}$ The protein level of RBBP7 was similarly reduced in HGPS patient cells (Fig. 1D, E). As previously observed for other nuclear defects in HGPS cells, the extent of reduction was variable amongst cells in the population, but its extent was similar to that observed in HGPS for several other nuclear proteins including all isoforms of the heterochromatin protein HP1. Furthermore, loss of RBBP4/7 occurred in the same cells that exhibited lower levels of HP1 pointing to global chromatin defects in those cells (Fig. 1D). ${ }^{2}$ Reduction of RBBP4 and RBBP7 in HGPS skin fibroblasts relative to control cells was confirmed by Western blot analysis on total cell lysates (Fig 1E). Similar observations were made in several independent primary HGPS patient cell lines (data not shown) and, as observed for HP1 $\gamma$, the extent of RBBP4/7 reduction increased during cell passaging (data not shown; ref. ${ }^{3-4}$ ). RBBP4 and RBBP7 down-regulation occurred at the protein level since similar levels of mRNAs were present in HGPS and control fibroblasts as determined by RT-PCR (Supplementary Fig. 2A). Consistent with the reduction of RBBP4/7, the levels of the centromeric protein CENP-A was reduced in HGPS cells (Supplementary Fig. 2B, 2C), in agreement with earlier observations demonstrating loss of CENP-A after concomitant siRNA silencing of RBBP4 and RBBP7. ${ }^{14}$ 
The loss of RBBP4 and RBBP7 was dependent on progerin. Upon controlled induction of a GFP-tagged version of progerin in immortalized wild-type skin fibroblasts using a tetracycline-dependent expression system ${ }^{6}$, RBBP4 and RBBP7 were almost completely lost within 4 days when compared with the uninduced control (Fig 1F). The control protein RAD52 was not affected by GFP-progerin expression (Fig. 1F). Conversely, elimination of progerin premRNA from HGPS skin fibroblasts by a previously characterized RNA morpholino approach ${ }^{2}$ rescued the levels of RBBP4 when compared to the same cells treated with a scrambled control oligonucleotide (Supplementary Fig. 2D). In addition, staining of HGPS primary fibroblasts with specific antiprogerin antibodies revealed that expression of progerin inversely correlated with RBBP4 levels in these cells (Supplementary Fig. 2E). Collectively, these results demonstrate loss of the histone chaperones RBBP4 and RBBP7 in HGPS cells in a progerin-dependent manner.

Given the role of RBBP4 and RBBP7 in various chromatin remodeling and assembly complexes, we hypothesized that their loss contributes to the changes in chromatin structure and elevated levels of DNA damage that occur during premature and normal aging. To directly test whether the absence of RBBP4/7 is sufficient to cause these defects, we analyzed the status of pericentromeric heterochromatin after siRNA-mediated knockdown of RBBP4 and RBBP7, either alone or in combination (Fig. 2). Efficient knockdown was confirmed by Western blotting (Supplementary Fig. 3A). As expected, cells treated with non-targeting control siRNA oligonucleotides displayed several bright foci of $\mathrm{H} 3 \mathrm{~K} 9 \mathrm{me} 3$, corresponding to pericentromeric heterochromatin ${ }^{15}$, as confirmed by partial colocalization of these foci with CREST antibody which recognizes centromeric proteins (Fig. 2A). Upon simultaneous knock-down of RBBP4 and RBBP7 the focal accumulation of H3K9me3 was lost (Fig. 2A). Silencing of either RBBP4 or RBBP7 alone was not sufficient to cause heterochromatin alterations (Fig. $2 A)$, most likely reflecting the functional overlap between these two highly similar proteins. In line with previous observations ${ }^{14}$ concomitant silencing of RBBP4 and RBBP7 led to loss of the centromeric protein CENP-A (Supplementary Fig. 3B). To further test whether RBBP4 and RBBP7 are involved in mediating the chromatin defects observed in HGPS cells, we probed the effect of loss of RBBP4 and RBBP7 on DNA SatIll repeat transcription by semi-quantitative RT-PCR (Fig. 2B). 
SatIII repeats are normally maintained in a transcriptionally silent state but are activated in HGPS cells, most likely as a result of heterochromatin disruption. ${ }^{4,}{ }^{16}$ As observed in HGPS cells, simultaneous silencing of RBBP4 and RBBP7 caused a significant increase in Satlll transcription compared to control siRNA treated cells (Fig. 2B).

To test whether loss of RBBP4 and RBBP7 is also sufficient to induce the increased levels of DNA damage present in HGPS cells, we probed RBBP4 and RBBP7 depleted cells for the presence of phosphorylated histone H2AX, a marker of DNA damage. ${ }^{17}$ Similar to cells from HGPS patients and aged individuals, the percentage of cells containing multiple prominent phospho$\mathrm{H} 2 \mathrm{AX}$ foci increased from $5 \%$ in control cells to more than $60 \%$ in cells depleted of RBBP4 and RBBP7 (Fig. 2C, D). ${ }^{3}$ The increase in endogenous DNA damage upon RBBP4/7 silencing was mirrored by an increased proportion of cells in S-phase as detected by FACS analysis (Supplementary Fig. 3C, D; P < 0.01 for Hela cells, $\mathrm{P}<0.01$ for U2OS cells), in agreement with the accumulation in S-phase of late-passage HGPS cells (Supplementary Fig. 3E, $P<0.01$ ). While the percentage of cells with aberrant chromatin structure in RNAi-treated cells was significantly increased $72 \mathrm{~h}$ after RNAi knockdown when compared to the control (Supplementary Fig. 4A), DNA damage only became evident at $120 \mathrm{~h}$ (Supplementary Fig. 4B), suggesting the occurrence of structural chromatin defects prior to DNA damage. Similar results were obtained after reduction of RBBP4/7 levels by induced expression of progerin (Supplementary Fig. 5).

RBBP4 and RBBP7 are shared subunits of multiple protein complexes involved in chromatin metabolism, including the NURD complex and the PRC2 complex. ${ }^{11-12}$ In addition, RBBP4 is an integral subunit of the CAF-1 complex. ${ }^{13}$ To test which of these complexes mediates the aging-associated chromatin defects caused by RBBP4 and RBBP7 loss, we tested the status of other subunits of the NURD, CAF-1 and PRC2 complexes in HGPS cells (Fig. 3). Similar to RBBP4 and RBBP7, two additional subunits of the NURD complex, MTA3 and HDAC1, were largely lost from HGPS cells as judged by quantitative single-cell microscopy and Western blotting (Fig. 3A-C). Consistent with a possible regulatory role of lamin A on the NURD complex, 


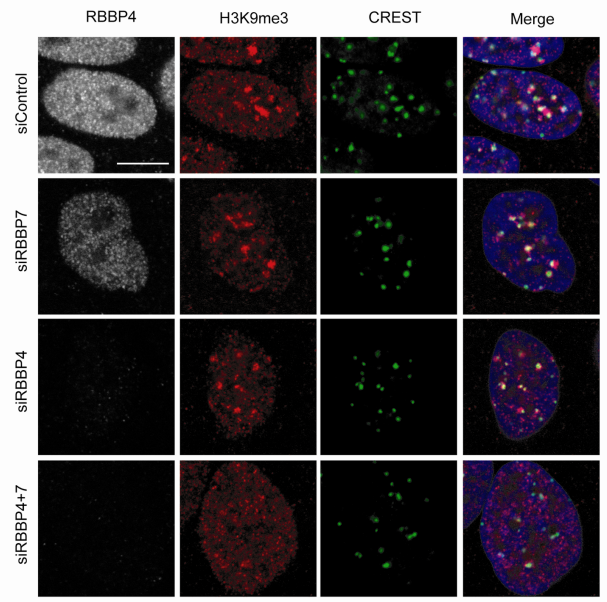

C

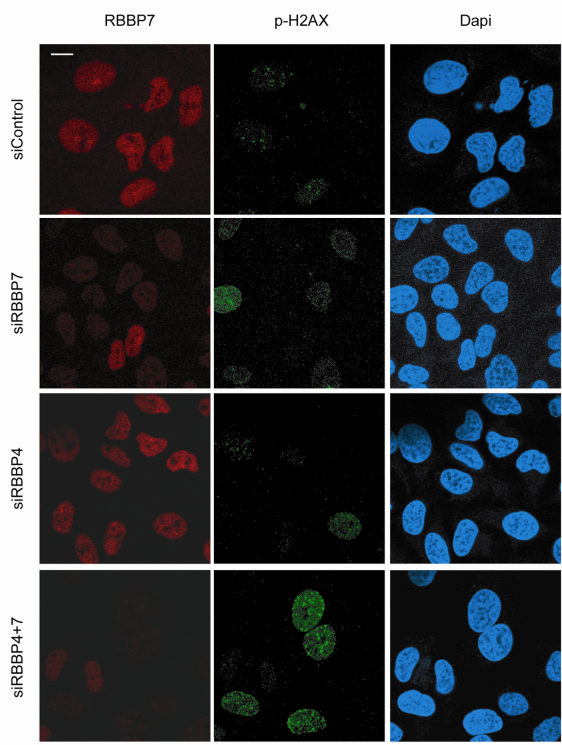

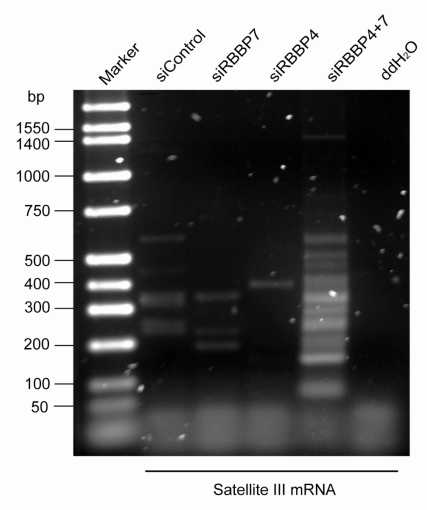

D

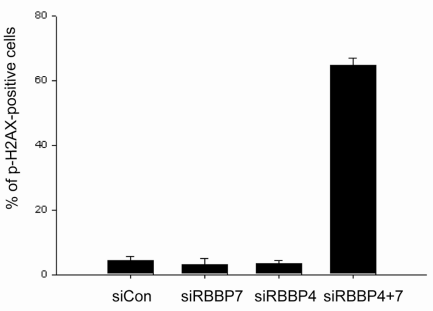

Fig. 2. Heterochromatin defects and increased DNA damage upon RBBP4 and RBBP7 silencing. (A) HeLa cells were transfected with the indicated combinations of siRNA for $144 \mathrm{hrs}$ and processed for immunofluorescence staining with the indicated antibodies. (B) Semi-quantitative, strand specific RT-PCR analysis of SatllI G-rich transcripts in cells knocked down for the indicated gene. (C) Detection of DNA damage in knock-down cells using $\alpha$-phospho-H2AX antibody. (D) Quantitative analysis of the percentage of $\mathrm{p}-\mathrm{H} 2 \mathrm{AX}$ positive cells (defined as containing more than 8 foci per cell).Values represent averages \pm S.D. from three experiments. $N>200$. Scale bars: $10 \mu \mathrm{m}$. 
A
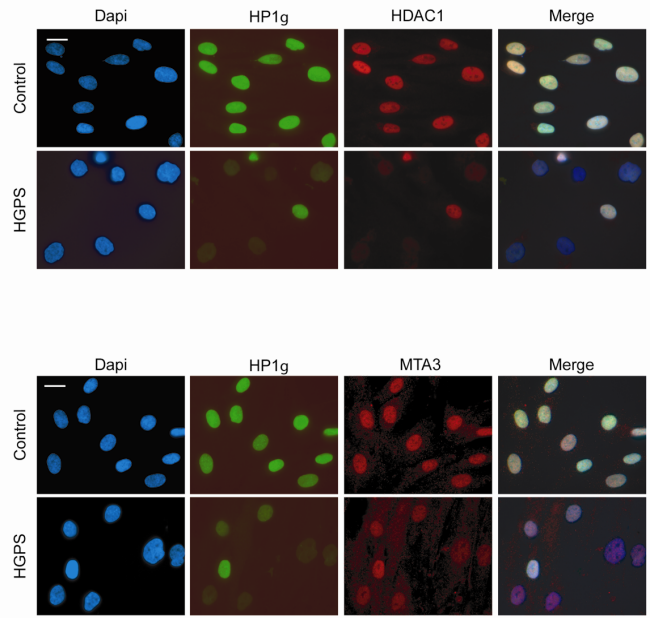

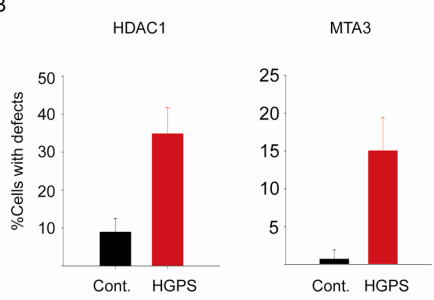

C

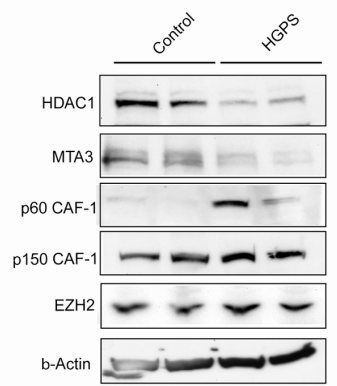

D
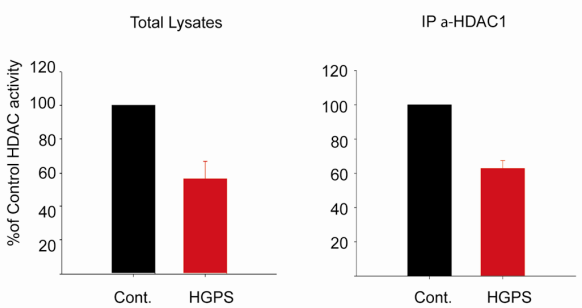

Fig 3. Loss of NURD subunits in HGPS cells. (A) Immunofluorescence staining of control and HGPS primary dermal fibroblasts with the indicated antibodies. (B) Quantitative analysis of the fluorescence intensity signal in control and HGPS cells. N>200; Values represent averages \pm S.D. from three experiments. (C) Western blot with the indicated antibodies on total cell lysates prepared from dermal fibroblast from two unaffected individuals and two HGPS patients. (D) HDAC activity measured in total lysates or in HDAC1 immunoprecipitates prepared from primary fibroblasts. Values represent averages \pm S.D. from three experiments. Scale bar: $10 \mu \mathrm{m}$. 
HDAC1 physically interacted with lamin $A$ in vivo as demonstrated by its immunoprecipitation with OST-lamin A (Supplementary Fig. 6A). In contrast, protein levels of $\mathrm{p} 150$ and $\mathrm{EZH} 2$, the catalytic subunit of the CAF-1 and the PRC2 complexes, respectively, were unaffected in HGPS cells (Fig. 3C). The p60 subunit of the CAF-1 complex was up-regulated in HGPS primary fibroblasts (Fig. 3 C), likely as a consequence of persistent DNA damage. ${ }^{18}$ We conclude the NURD complex is lost from HGPS patient cells.

The loss of HDAC1 protein in HGPS cells pointed to the possibility that the observed chromatin defects are caused by loss of cellular HDAC1 deacetylase activity. To test this hypothesis we measured HDAC1 activity in HGPS patient cells. HDAC1 activity in total cell extracts and in HDAC1 immunoprecipitates prepared from HGPS patient cells was reduced by $40 \%$ compared to matched control cells (Fig. 3D). To directly probe whether the NURD complex is responsible for the aging-associated chromatin defects, we individually knocked down the NURD subunits HDAC1, MTA3, CHD3 or CHD4 in Hela cells (Supplementary Fig. 6B). In a manner similar to RBBP4/7 knock down, silencing of any subunit increased the percentage of cells lacking H3K9me3 and HP1 $\gamma$ heterochromatin foci (Fig. 4A, B). Furthermore, shRNA mediated silencing of HDAC1, MTA3, CHD3 or CHD4 in primary human fibroblasts (Supplementary Fig. 6C, D) increased the percentage of cells containing phospho-H2AX positive foci to similar levels as observed in HGPS cells (Fig. 4C, D). These results demonstrate that HDAC1 activity is reduced in HGPS cells and that loss of NURD complex components is sufficient to recapitulate some aging-associated chromatin defects.

Since chromatin defects associated with HGPS also occur during physiological aging ${ }^{3}$, we finally probed the status of the NURD subunits in cells from normally aged individuals. Quantitative immunofluorescence microscopy analysis on cells obtained from two young donors ( 7 and 9 years, respectively) and passage-matched primary fibroblasts from two old donors (92 and 88 years, respectively), revealed significant differences in RBBP4, RBBP7, and HDAC1 protein levels (Fig 5A-C). Cell populations from old individuals consistently showed a 4-5 fold increase in the percentage of cells with reduced protein levels of RBBP4, RBBP7 and HDAC1 (Fig. 5D). At the single cell level, the extent of reduction of NURD components strictly 
A

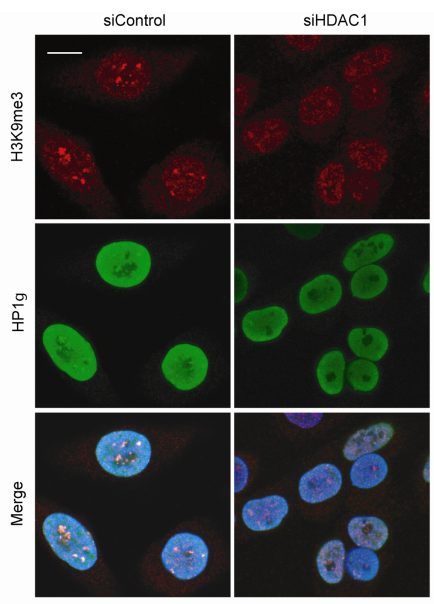

B

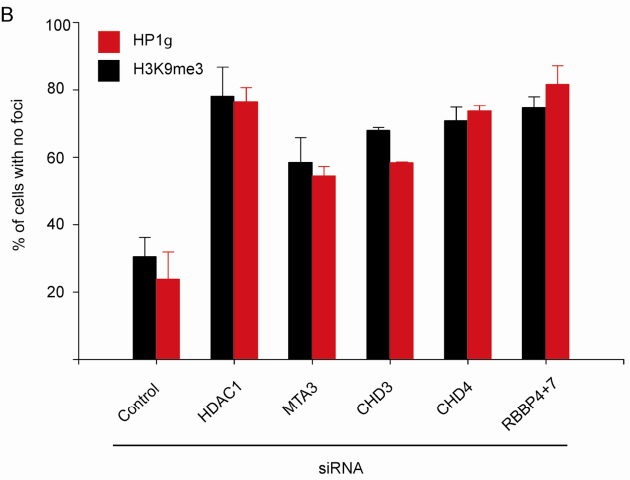

C

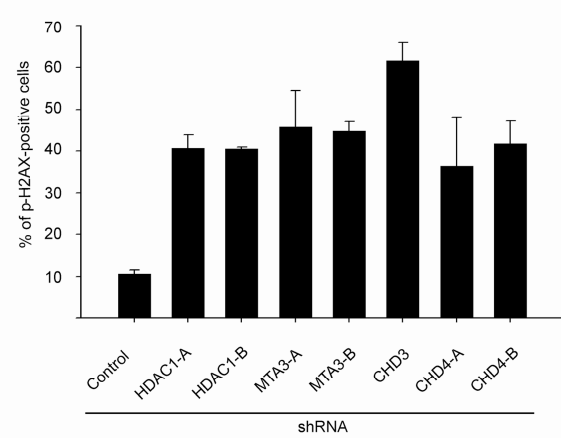

Fig. 4. Heterochromatin defects and increased DNA damage upon silencing of NURD subunits. (A) HeLa cells were transfected with the indicated siRNA for $96 \mathrm{hrs}$ and processed for immunofluorescence staining with the indicated antibodies. Scale bar: $10 \mu \mathrm{m}$. (B) Quantitation of the percentage of siRNA treated Hela cells in which $\mathrm{H} 3 \mathrm{~K} 9 \mathrm{me} 3$ or HP1 $\gamma$ foci are absent. Values represent averages \pm S.D. from two experiments. $N>200$. (C) Quantitation of the percentage of phospho-H2AX positive cells (defined as containing more than 8 foci per cell).Values represent averages \pm S.D. from three experiments. $N>200$. Scale bar: $10 \mu \mathrm{m}$. 
correlated with each other and with HP1 $\gamma$ levels, albeit less pronounced than that observed for HGPS cells as previously described ${ }^{3}$. In contrast to HGPS cells, no loss of CENP-A was detected in normally aged cells. We conclude that loss of the RBBP4, RBBP7, and HDAC1 subunits of NURD is not limited to premature aging but is also a feature of physiologically aged cells. Here we have identified the NURD chromatin remodeling complex as a mediator of aging-associated chromatin defects. We demonstrate reduction of several NURD subunits in cells from a progeroid syndrome and from normally aged individuals. Loss of any one of these components and reduction of HDAC1 activity is sufficient to recapitulate multiple agingassociated chromatin defects. The in vivo physical interaction of lamin $A$ with RBBP4, RBBP7 and HDAC1 observed here, and previously suggested by biochemical fractionation ${ }^{19}$, points towards a regulatory role for the nuclear lamina in the function of the NURD complex in normal cells.

We find that loss of RBBP4/7 is dependent on the presence of progerin and is an early event in the appearance of aging-associated chromatin defects. Upon induction of progerin or upon knock-down of RBBP4 and RBBP7, changes to heterochromatin structure occur and are followed by accumulation of DNA damage. We suggest that loss of critical chromatin components RBBP4/7 is an upstream event in the formation of agingassociated chromatin defects. Loss of RBBP4/7 compromises the establishment and maintenance of histone modifications and higher order chromatin structure, possibly making chromatin more susceptible to DNA damage. The increase in DNA damage might be a consequence of higher susceptibility of the affected chromatin to damage or alternatively due to impaired DNA replication. ${ }^{20}$ Interestingly, similar to the effects we observe upon silencing of RBBP4 and RBBP7, impairment of the H4K20 histone methyltransferase PR-Set7, which is necessary for proper H4K2O trimethylation associated with heterochromatin, interferes with DNA replication, arrests cells in S-phase and causes increased levels of DNA damage. ${ }^{21-22}$

Our finding that multiple NURD components are lost in HGPS and normally aged cells suggests that the integrity of the NURD complex is compromised and its components are down-regulated during premature and physiological aging. Whether loss of these proteins occurs by activation of a specific 
A
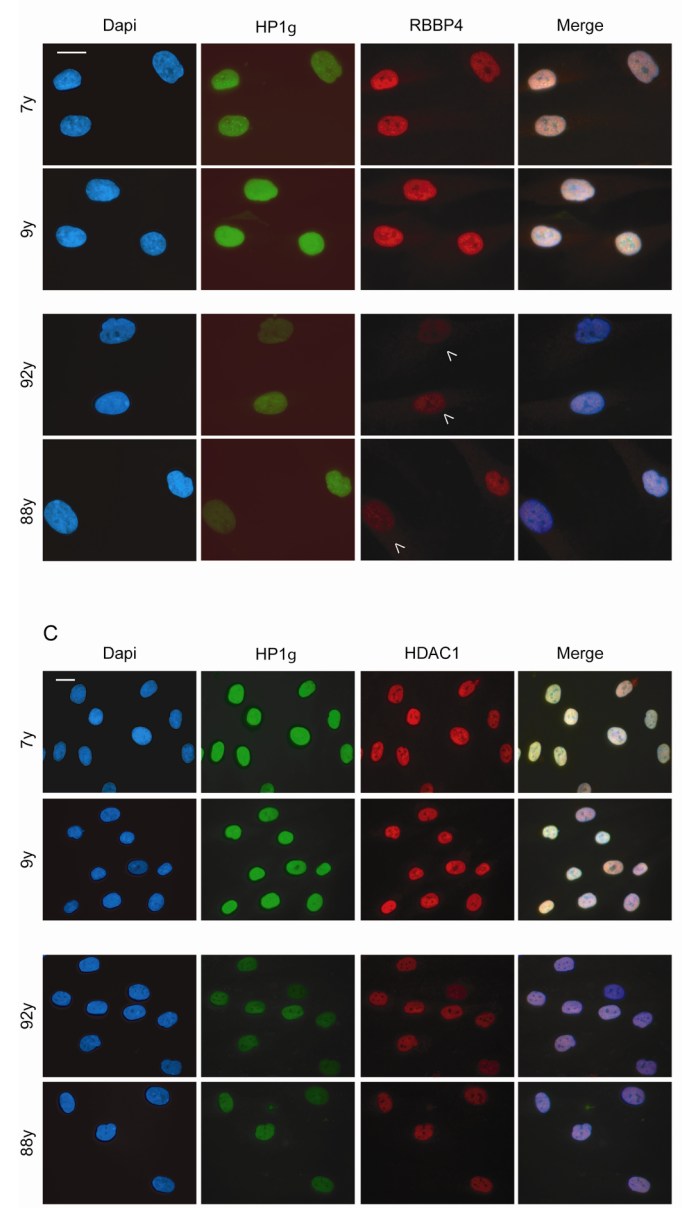

B
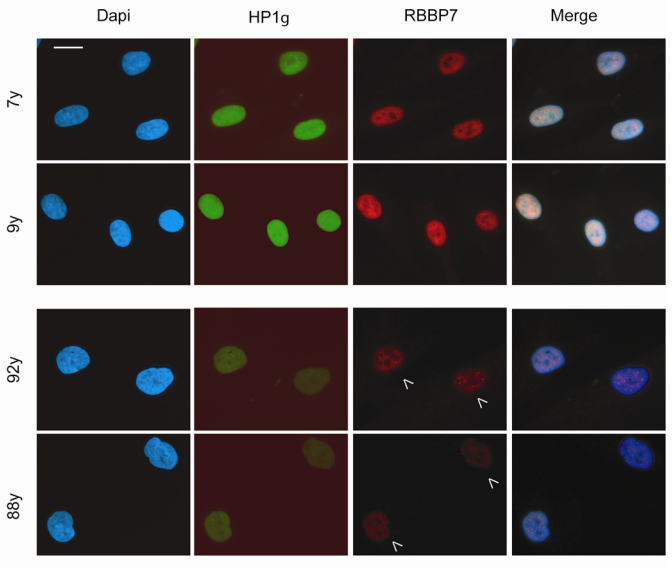

D

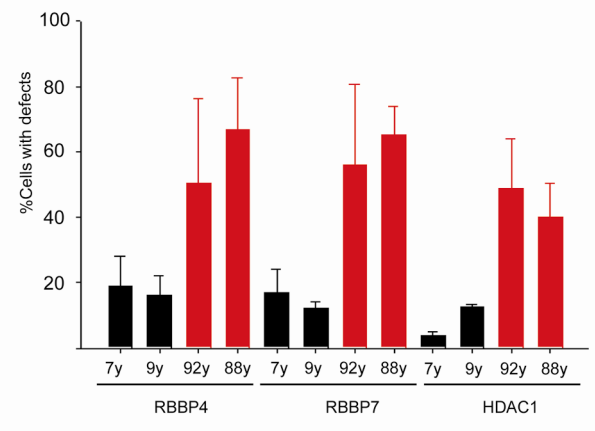

Fig 5. Loss of NURD subunits in physiological aging. (A), (B) and (C) Immunofluorescence microscopy of passage-matched primary dermal fibroblasts from healthy young ( $7 \mathrm{yrs}$ and $9 \mathrm{yrs}$ ) or old (92 yrs and 88 yrs) donors were fixed and stained with the indicated antibodies. (D) Quantitative analysis of the fluorescence intensity signal measured in dermal fibroblasts from young and old individuals. $N>200$; Values represent averages \pm S.D. from three experiments. Arrowheads indicate cells with reduced levels of heterochromatin proteins. Scale bars: $10 \mu \mathrm{m}$. 
protein degradation program or by interference with the normal homeostasis of the NURD protein complex remains to be determined. The involvement of a specific pathway is suggested by the fact that progerin affects the protein levels of several subunits of the NURD complex, but not of other RBBP4- or RBBP7-containing complexes, such as CAF-1 or PRC2. While we cannot rule out that the degradation mechanism of NURD components are distinct in HGPS cells and in normally aged cells, the fact that progerin is also expressed at low levels in cells from normally aged individuals is consistent with a conserved degradation mechanism. ${ }^{3}$ Overall, these results implicate declining NURD remodeling complex function in aging-associated chromatin defects and accumulation of DNA damage during aging.

\section{Materials and Methods}

\section{Cell culture}

Primary dermal fibroblast cell lines were obtained from the NIA and NIGMS collections of the Coriell Cell Repository (CCR), from the American Type Culture Collection (ATCC) or the Progeria Research Foundation (PRF). The cells from HGPS patients were AG01972 (Patient age 14 years, CCR, population doublings (PD) 20 at purchase), AG06297 (8 years, CCR, PD17), HGDAFN003 (2 years, PRF, PD7). Control cell lines were CRL-1474 (7 years, ATCC, PD19) GM00038 (9 years, CCR, PD17), AG05247 (88 years, CCR, PD15) and AG09602 (92 years, CCR, PD 12). Cells were used at PD's 19-27. WI-38 primary human fetal fibroblasts ( 3 month gestation fetus, ATCC, PD8)were used at PD's 10-18. All primary fibroblasts were grown in Minimum Essential Medium (MEM, Gibco) , 15\% FCS, 2mM L-glutamine, $100 \mathrm{U} / \mathrm{ml}$ penicillin and $100 \mu \mathrm{g} / \mathrm{ml}$ streptomycin at $37{ }^{\circ} \mathrm{C}$ in $5 \% \mathrm{CO}_{2}$. Growth and induction of immortalized, Tet-off human fibroblasts expressing GFP-Progerin was as previously described ${ }^{6}$. U2OS cells stably expressing One-Strep-Lamin A were maintained in McCoy's 5A medium (Gibco), 10\% FCS, 2mM L-glutamine, 10 $\mu \mathrm{g} / \mathrm{ml}$ blasticidin at $37{ }^{\circ} \mathrm{C}$ in $5 \% \mathrm{CO}_{2}$. 


\section{Plasmids}

Plasmids expressing GST -RBBP4/7 were a kind gift of B. Stillman (Cold Spring Harbor, NY ${ }^{10}$. Vectors for in vitro transcription and translation of lamin $A$ and progerin were constructed as follows: an EcoRI/BamHI fragment containing the entire CDS of lamin A (1-664) or progerin was excised from either pEGFP-Lamin A or pEGFP-Lamin $\Delta 50^{2}$, respectively, and cloned into the pCDNA3.1 (-) vector (Invitrogen) cut with the same enzymes. pGBKT7-lamin A (390-646) was a kind gift of T. Dittmer (NCI/NIH, Maryland). $\mathrm{N}$-terminal One-Strep tag (IBA BioTagnology, Göttingen, Germany) oligonucleotides (Sigma, Zwijndrecht, Netherlands) were cloned into BamHI and EcoRI sites of pBabe puro (Plasmid 1764, Addgene). The retroviral pBabe puro One-Strep-Lamin A plasmid was generated by inserting lamin $A$ from the pBabe puro HA-Lamin A vector ${ }^{23}$ into the Sall and EcoRI sites. In order to generate a lentiviral expression system a BamHI and EcoRI containing multiple cloning site was introduced in the pCDH1-MCS1-EF1Puro vector (System BioSciences, Mountainview, California, USA), the puromycin cassette was replaced by a blasticidin cassette from the pLenti6/V5-GW/LacZ vector (Invitrogen, Carlsbad, California, USA) and the One-STrEP-tagged lamin A from the $\mathrm{pBabe}$ puro One-Strep-Lamin A vector was ligated into the BamHI and EcoRI restriction sites, thereby generating the lentiviral pCDHblast MCSNard OST-Lamin A vector. The Yeast Two Hybrid screen was performed as described ${ }^{24-25}$

\section{SiRNA and shRNA constructs}

siGenome SMART pool siRNA oligos against human RBBP4 and RBBP7 were purchased from Dharmacon-Thermo Scientific and transfected at 0 and $72 \mathrm{~h}$ into Hela cells at $50 \mathrm{nM}$ using Oligofectamine (Invitrogen) or Dharmafect1 (Dharmacon-Thermo), following the manufacturer instructions. RBBP4:

$\begin{array}{ll}\text { Target 1: } & \text { 5'-GAUACUCGUUCAAACAAUA } \\ \text { Target2: } & \text { 5'-GAACUGCCUUUCUUUCAAU } \\ \text { Target3: } & \text { 5'-GGAUACUCGUUCAAACAAU } \\ \text { Target4: } & \text { 5'-AACAAUACUUCCAAACCAA } \\ \text { RBBP7: } & \\ \text { Target1: } & \text { 5'-GGAUAAGACCGUAGCUUUA } \\ \text { Target2: } & \text { 5'-CCACUGGUCUCCACAUAAU }\end{array}$


Target3: 5'-GGACACACUGCUAAGAUUU

Target4: 5'-AAGUAAACCGUGCUCGUUA

HDAC1:

Target1: 5'-CUAAUGAGCUUCCAUACAA

Target2: 5'-GAAAGUCUGUUACUACUAC

Target3: 5'-GGACAUCGCUGUGAAUUGG

Target4: 5'-CCGGUCAUGUCCAAAGUAA

MTA3:

Target1: 5'-GCAGAAACAUCAGUUGAAA

Target2: 5'-UGACUAGCAUCAUUGAAUA

Target3: 5'-CUUCAAUGACAUACGGCAA

Target4: 5'-GUGCAACAGAAACGUCUAA

CHD3:

Target1: 5'-CGUAUGAGCUGAUCACCAU

Target2: 5'-GAGGAGAAGUACUAUCGUU

Target3: 5'-GAGGCACCCUGCACAUGUA

Target4: 5'-CAAGAUAGAUCAUAAGUUG

CHD4:

Target1: 5'-CCAAGGACCUGAAUGAUGA

Target2: 5'-CAAAGGUGCUGCUGAUGUA

Target3: 5'-GAAAGAGGCAUCUGUGAAA

Target4: 5'-GAUGAUAUCCUCAAAUUUG

GIPZ lentiviral vectors expressing shRNAmir sequences targeted against human HDAC1, MTA3, CHD3 and CHD4 were purchased from Open Biosystems-Thermo Scientific (Waltham, MA) and virus produced in 293FT packaging cells (Invitrogen) usingFUGENEHD (Roche), following the manufacturer's instructions. Target W138 fibroblasts ( 50\% confluent) were incubated for $8 \mathrm{hrs}$ with a 1:1 mix of filtered viral supernatant and $\mathrm{MEM}+15 \% \mathrm{FBS}$ and in the presence of $6 \mu \mathrm{g} / \mathrm{ml}$ Polybrene (Sigma-Aldrich). A second round of infection with fresh lentiviral vector-containing supernatant was performed after $24 \mathrm{hrs}$. 48-72 hrs after the first round of infection, puromycin $(2 \mu \mathrm{g} / \mathrm{ml})$ was added to the medium to select shRNAmir expressing cells. Experiments were performed 6 days after the first round of infection. 
GIPZ non-targeting sequence: 5'-TCTCGCTTGGGCGAGAGTAAG

HDAC1-A: 5'-CCCGAATCCGCATGACTCATAA

HDAC1-B: 5'-AACCCATTCTTCCCGTTCTTAA

MTA3-A: 5'-CCCTAATATGCAGTGTAGATTA

MTA3-B: 5'-CCGGCCGTTTGTTGCTATTAAT

CHD3: 5'-AGGAATTACCACTATCTAGTAA

CHD4-A: 5'-CGCTGCTGACATCCTATGAATT

CHD4-B: 5'-CGCCCTCCAAGACAGAACTAAT

\section{Antibodies}

The following antibodies were used in immunofluorescence, Western blotting and immunoprecipitation: $\alpha-\mathrm{HP} 1 \gamma$ mouse monoclonal (MAB3450, Chemicon), $\alpha$ - H3K9me3 rabbit polyclonal (4861, kindly provided by $\mathrm{T}$. Jenuwein), $\alpha$-RBBP4 mouse monoclonal (11G10, Abcam), $\alpha$-RBBP4 rabbit polyclonal (ab1765, Abcam), $\alpha$-RBBP7 rabbit polyclonal (ab3535, Abcam), $\alpha$-RAD52 rabbit polyclonal (3425, Cell Signaling), $\alpha-p-H 2 A X$ mouse monoclonal (JBW301, Millipore), $\alpha-\mathrm{p}-\mathrm{H} 2 \mathrm{AX}$ mouse monoclonal (2F3, Abcam), $\alpha$-HDAC1 rabbit polyclonal (PA1-860, ABR), MTA3 rabbit polyclonal (A300-160A, Bethyl Laboratories), $\alpha-p 150$ CAF-1 mouse monoclonal SS1 and $\alpha$-p60 CAF- 1 mouse monoclonal SS53 (kind gifts of $B$. Stillman), $\alpha$-EZH2 rabbit polyclonal (39103, Active Motif), $\alpha$-CHD3 rabbit polyclonal (A301219A, Bethyl Laboratories), $\alpha$-CHD4 rabbit polyclonal (H-242, St. Cruz), $\alpha-$ CHD4 mouse monoclonal (91984, Genetex), $\alpha$-Lamin A goat polyclonal ( $N$ 18 , St. Cruz), $\alpha$-progerin mouse monoclonal 13A4, (Alexis Biochemicals), $\alpha$ centromeric proteins CREST human autoimmune serum (Antibodies Inc.), $\alpha$ CENP-A mouse monoclonal (3-19, Abcam).

\section{Immunofluorescence microscopy and image quantitation}

Indirect immunofluorescence microscopy was performed as previously described ${ }^{2}$. Quantitative microscopy measurements were performed as previously described ${ }^{2-3}$. For single cell analysis, at least 200 cells per sample were counted in triplicate and the error bars in the histograms represent the standard deviation. 


\section{Western blotting}

Cells were rinsed in PBS and lysed in either $1 \mathrm{X}$ Laemmli SDS-PAGE loading buffer or, alternatively, in a buffer containing $150 \mathrm{mM} \mathrm{NaCl}, 20 \mathrm{mM}$ Hepes pH 7.4, 0.5\% NP40, 1mM EDTA, $10 \mathrm{mM} \beta$-Glycero-phosphate, $1 \mathrm{mM} \mathrm{NaF}, 1$ $\mathrm{mM} \mathrm{Na} \mathrm{VO}_{4}$, Protease Inhibitors Cocktail SetIll (Calbiochem). Western blotting and immunodetection were performed as previously described ${ }^{2}$.

\section{Recombinant proteins expression and in vitro binding assays}

GST-fusion proteins were expressed in E. coli BL21(DE3)pLysS (Promega) and affinity purified on glutathione Sepharose beads (GE Healthcare). For in vitro binding assays, GST fusion proteins (1-5 $\mu \mathrm{g})$ immobilized on glutathione Sepharose beads were pre-washed twice in NETN150 $(20 \mathrm{mM}$ Tris- $\mathrm{HCl}$ pH 7.5, $150 \mathrm{mM} \mathrm{NaCl}, 1 \mathrm{mM}$ EDTA, 0.5\% NP40, $1 \mathrm{mM} \mathrm{DTT}$ ), incubated $30 \mathrm{~min}$ at $4^{\circ} \mathrm{C}$ with Benzonase, washed once in NETN1000, blocked for $30 \mathrm{~min}$ in NETN150, $1 \mathrm{mg} / \mathrm{ml}$ BSA and then washed twice in NETN150. $\left[{ }^{35} \mathrm{~S}\right]$ - labelled proteins were generated by in vitro transcription/translation using a T7TNTQuick-rabbit reticulocyte lysate system (Promega). Equal amounts of in vitro translated, $\left[{ }^{35} \mathrm{~S}\right]$-labelled proteins were incubated in a total volume of $50 \mu \mathrm{L}$ of NETN150 with either GST or the appropriate GST fusion protein for $1 \mathrm{hr}$ at $4^{\circ} \mathrm{C}$ in binding buffer. The beads were washed 6x with NETN150, boiled for $5 \mathrm{~min}$ in 1x SDS-PAGE Laemmli loading buffer, and resolved on a SDS-PAGE gel. The gel was dried and $\left[{ }^{35} \mathrm{~S}\right]$ - labelled proteins were visualized using a Storm 860 (Molecular Dynamics).

\section{RNA extraction and RT-PCR}

RNA was extracted from cells using the RNeasy Mini Kit (QIAGEN) following the manufacturer instructions. Residual contaminating genomic DNA was eliminated by treating the RNA with Turbo DNA-free DNAse (Ambion). RBBP4 and RBBP7 mRNA levels in control and HGPS cells were measured by retrotranscribing $1 \mu \mathrm{g}$ of RNA using random primers and the Mo-MulV RT enzyme included in the High Capacity Archive cDNA kit (Applied Biosystems) for $2 \mathrm{hr}$ at $42{ }^{\circ} \mathrm{C}$, after a denaturation step of $5 \mathrm{~min}$ at $65^{\circ} \mathrm{C}$. Equal volumes of cDNA were employed as template in a real-time Q-PCR reactions using iQ SYBR Green Supermix (Biorad) in a Biorad iCycler. Reaction conditions were: $3 \mathrm{~min}$ at $95{ }^{\circ} \mathrm{C}, 1 \mathrm{cycle} ; 20 \mathrm{sec}$ at $95{ }^{\circ} \mathrm{C}, 30 \mathrm{sec}$ at $56{ }^{\circ} \mathrm{C}, 41 \mathrm{cycles}$. Melting 
curves of the amplified product were generated to verify that a single amplicon was generated. Primers :

RBBP4-6-Fw (5'-TCTGTTTGGGTCAGTTGCTG-3')

RBBP4-6-Rw (5'-AACTGAGTGGCTTGGTTTGG -3')

RBBP7-6-Fw (5'-CTGGCCACTCAGCTGTTGTA -3')

RBBP7-6-Rw (5'-AGGTGGTATTGGACCTGGTG -3').

All the values were normalized to the internal control Cyclophilin A gene: Cycl-Fw (5'-GTCAACCCCACCGTGTTCTT-3') and Cycl-Rw (5'CTGCTGTCTTTGGGACCTTGT $-3^{\prime}$ ). The G-rich strand of Satlll transcripts was retrotranscribed and PCR amplified as previously described ${ }^{26}$.

\section{HDAC1 immunoprecipitation and HDAC assays}

CRL- 1474 and AG01972 primary fibroblasts were grown in $10 \mathrm{~cm}$ dishes to 70-90\% confluency. Cells were rinsed in PBS and resuspended in Lysis buffer (300 mM NaCl, 50 mM Hepes pH 7.4, 0.5\% NP40, 1 mM EDTA, 20\% glycerol, $10 \mathrm{mM} \beta$-Glycero-phosphate, $1 \mathrm{mM} \mathrm{NaF}, 1 \mathrm{mM} \mathrm{Na}_{3} \mathrm{VO}_{4}$, Protease Inhibitors Cocktail SetIII (Calbiochem)). The cell lysate was incubated for $30 \mathrm{~min}$ at $4{ }^{\circ} \mathrm{C}$ on a rotating wheel and then centrifuged for $12 \mathrm{~min}$ at $12000 \mathrm{~g}$. Total protein concentration of lysates was measured using the Bradford Assay reagent (Biorad). Equal quantities of protein lysate from different samples (1.5-2.0 $\mathrm{mg}$ ) were diluted to $1 \mathrm{ml}$ with Lysis buffer. Lysates were pre-cleared by incubation for $1 \mathrm{hr}$ at $4{ }^{\circ} \mathrm{C}$ with rabbit-preimmune serum and Protein $\mathrm{A} / \mathrm{G}+$ agarose (St. Cruz) and then incubated $\mathrm{o} / \mathrm{n}$ at $4{ }^{\circ} \mathrm{C}$ with a $\alpha-\mathrm{HDAC} 1$ rabbit polyclonal antibody. Immunocomplexes where captured by adding Protein A/G+ agarose. Beads were washed $5 \mathrm{X}$ in Lysis buffer and rinsed twice in HDAC assay buffer (see below). HDAC activity in total lysates and in HDAC1 immunoprecipitates was measured with the Fleur de Lys Fluorescent HDAC assay kit (Biomol) following the manufacturer instructions.

\section{OneSTrEP-Iamin A pulldown}

U2OS cells stably expressing OneSTrEP-lamin A were grown in $150 \mathrm{~mm}$ dishes, fixed in formhaldeyde and lysed in SDS lysis buffer (1\% SDS, $10 \mathrm{mM}$ EDTA, $50 \mathrm{mM}$ Tris $\mathrm{pH}$ 8.1). After extensive sonication, the SDS concentration in the lysate was lowered 10 -fold by addition of SDS dilution buffer $(0.01 \%$ SDS, 1.1 \% Triton X-100, $1.2 \mathrm{mM}$ EDTA, $17 \mathrm{mM}$ Tris $\mathrm{pH} 8.1,170 \mathrm{mM} \mathrm{NaCl}$ ). 
Both SDS lysis buffer and SDS dilution buffer were supplemented with $2 \mathrm{mM}$ ortohovanadate and protease inhibitors (Boehringer Mannheim, Indianapolis, USA). Undissolved fractions were discarded after a 10 minute centrifugation step at 4 degrees at 10,000xg. Streptactin Matrix (IBA BioTagnology, Göttingen, Germany) prewashed in SDS dilution buffer was added and samples were incubated on a rotating wheel $\mathrm{o} / \mathrm{n}$ at $4{ }^{\circ} \mathrm{C}$. Precipitated protein complexes were washed extensively, and eluted from the beads by incubation at $95^{\circ} \mathrm{C}$ in $1 \mathrm{X}$ Laemmli SDS-PAGE loading buffer.

\section{Acknowledgements}

We would like to thank P. Scaffidi and A. Marcello for critical reading of the manuscript and P. Scaffidi for sharing data and providing numerous suggestions during the course of the experiments. Fluorescence imaging was performed with the assistance of T. Karpova at the $\mathrm{NCl}$ Fluorescence Imaging Microscopy Facility. FACS cell cycle acquisition and analysis was performed with the help of K. McKinnon at the NCI FACS facility. We would also like to thank T. Jenuwein, B. Stillman and T. Dittmer for providing reagents. We thank $\mathrm{H}$. Herrmann and E. Wanker for their support with the $\mathrm{Y} 2 \mathrm{H}$ screening and subsequent confirmation studies. G.P. was partly supported by postdoctoral fellowships from the Telethon Italy Foundation and the Italian Association for Cancer Research (AIRC). K.H. and H.G. were supported by The Deutsche Forschungsgemeinschaft (DFG, SFB 577, projects A4 and Z2). This work was supported by an Intramural Research Program of the $\mathrm{NIH}, \mathrm{NCl}$, Center for Cancer Research. 


\section{References}

1. Goldman, R.D., et al., Accumulation of mutant lamin A causes progressive changes in nuclear architecture in Hutchinson-Gilford progeria syndrome. Proc Natl Acad Sci U S A, 2004. 101(24): p. 8963-8.

2. Scaffidi, P. and T. Misteli, Reversal of the cellular phenotype in the premature aging disease Hutchinson-Gilford progeria syndrome. Nat Med, 2005. 11(4): p. 440-5.

3. Scaffidi, P. and T. Misteli, Lamin A-dependent nuclear defects in human aging. Science, 2006. 312(5776): p. 1059-63.

4. Shumaker, D.K., et al., Mutant nuclear lamin A leads to progressive alterations of epigenetic control in premature aging. Proc Natl Acad Sci U S A, 2006. 103(23): p. 8703-8.

5. Liu, B., et al., Genomic instability in laminopathy-based premature aging. Nat Med, 2005. 11(7): p. 780-5.

6. Scaffidi, P. and T. Misteli, Lamin A-dependent misregulation of adult stem cells associated with accelerated ageing. Nat Cell Biol, 2008. 10(4): p. 452-9.

7. Capell, B.C. and F.S. Collins, Human laminopathies: nuclei gone genetically awry. Nat Rev Genet, 2006. 7(12): p. 940-52.

8. Eriksson, M., et al., Recurrent de novo point mutations in lamin A cause HutchinsonGilford progeria syndrome. Nature, 2003. 423(6937): p. 293-8.

9. De Sandre-Giovannoli, A., et al., Lamin a truncation in Hutchinson-Gilford progeria. Science, 2003. 300(5628): p. 2055.

10. Verreault, A., et al., Nucleosomal DNA regulates the core-histone-binding subunit of the human Hat1 acetyltransferase. Curr Biol, 1998. 8(2): p. 96-108.

11. Zhang, Y., et al., Analysis of the NuRD subunits reveals a histone deacetylase core complex and a connection with DNA methylation. Genes Dev, 1999. 13(15): p. 192435.

12. Kuzmichev, A., et al., Histone methyltransferase activity associated with a human multiprotein complex containing the Enhancer of Zeste protein. Genes Dev, 2002. 16(22): p. 2893-905.

13. Verreault, A., et al., Nucleosome assembly by a complex of CAF-1 and acetylated histones H3/H4. Cell, 1996. 87(1): p. 95-104.

14. Hayashi, T., et al., Mis16 and Mis18 are required for CENP-A loading and histone deacetylation at centromeres. Cell, 2004. 118(6): p. 715-29.

15. Peters, A.H., et al., Partitioning and plasticity of repressive histone methylation states in mammalian chromatin. Mol Cell, 2003. 12(6): p. 1577-89.

16. Rizzi, N., et al., Transcriptional activation of a constitutive heterochromatic domain of the human genome in response to heat shock. Mol Biol Cell, 2004. 15(2): p. 543-51.

17. Rogakou, E.P., et al., DNA double-stranded breaks induce histone H2AX phosphorylation on serine 139. J Biol Chem, 1998. 273(10): p. 5858-68.

18. Nabatiyan, A., D. Szuts, and T. Krude, Induction of CAF-1 expression in response to DNA strand breaks in quiescent human cells. Mol Cell Biol, 2006. 26(5): p. 1839-49.

19. Cronshaw, J.M., et al., Proteomic analysis of the mammalian nuclear pore complex. J Cell Biol, 2002. 158(5): p. 915-27.

20. Peng, J.C. and G.H. Karpen, Epigenetic regulation of heterochromatic DNA stability. Curr Opin Genet Dev, 2008. 18(2): p. 204-11.

21. Jorgensen, S., et al., The histone methyltransferase SET8 is required for S-phase progression. J Cell Biol, 2007. 179(7): p. 1337-45. 
22. Tardat, M., et al., PR-Set7-dependent lysine methylation ensures genome replication and stability through S phase. J Cell Biol, 2007. 179(7): p. 1413-26.

23. Van Berlo, J.H., et al., A-type lamins are essential for TGF-beta1 induced PP2A to dephosphorylate transcription factors. Hum Mol Genet, 2005. 14(19): p. 2839-49.

24. Goehler, H., et al., A protein interaction network links GIT1, an enhancer of huntingtin aggregation, to Huntington's disease. Mol Cell, 2004. 15(6): p. 853-65.

25. Stelzl, U., et al., A human protein-protein interaction network: a resource for annotating the proteome. Cell, 2005. 122(6): p. 957-68.

26. Valgardsdottir, R., et al., Transcription of Satellite III non-coding RNAs is a general stress response in human cells. Nucleic Acids Res, 2008. 36(2): p. 423-34. 


\section{Supplemental Figures}

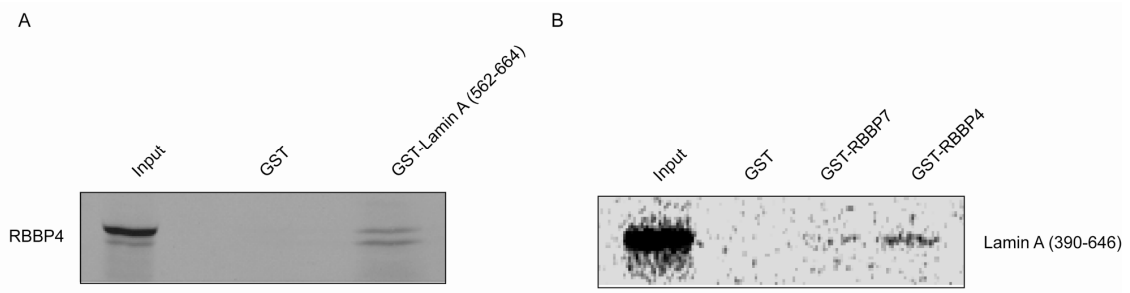

Figure S1. Validation of the RBBP4 interaction with lamin A (aa 562-664) by GST pull-down assay. (A) GST pulldown assays with recombinant GST-lamin A (aa 562-664) and ${ }^{35}$ S-labelled, in vitro transcribed and translated RBBP4. (B) GST pulldown assays with recombinant GSTRBBP4 or RBBP7 and ${ }^{35}$ S-labelled, in vitro transcribed and translated lamin A (aa 390-646). 
A

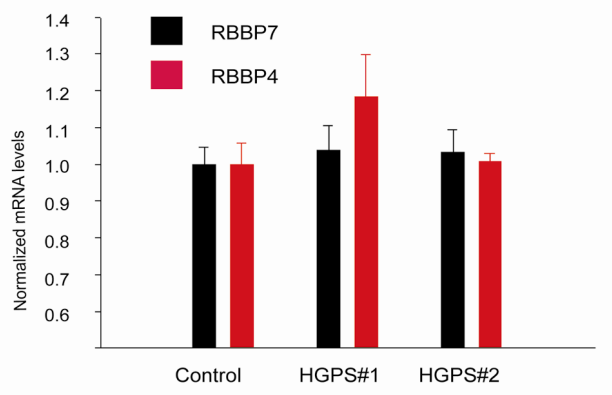

B

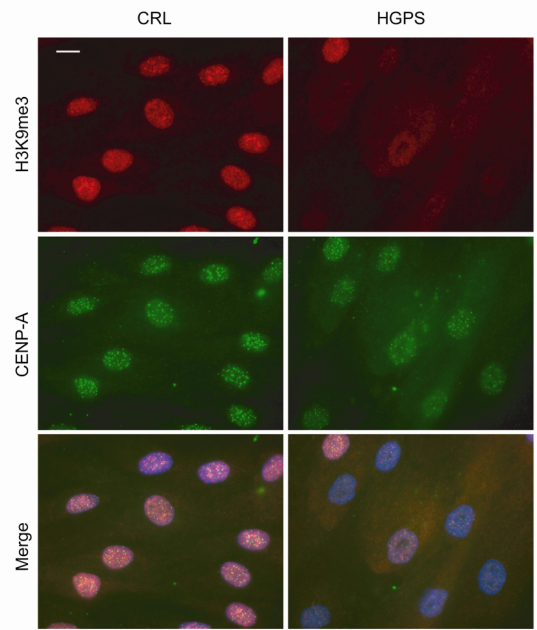

C

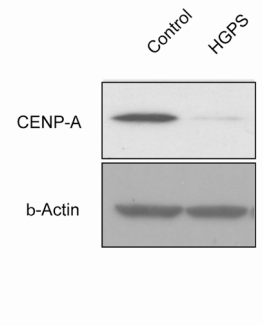

D

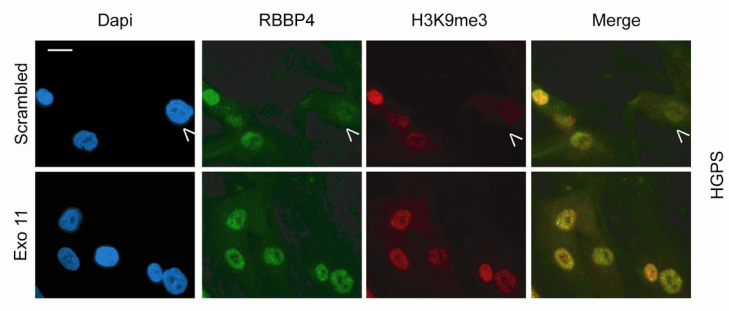

E
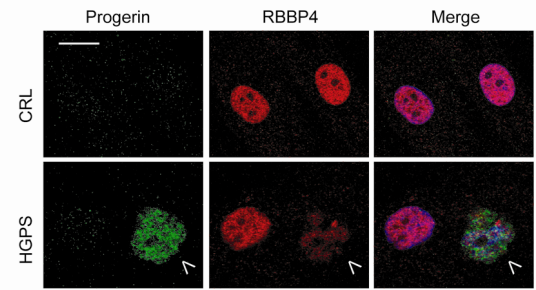

Figure S2. Characterization of RBBP4 and 7 reduction. (A) Quantitative RT-PCR analysis of RBBP4 and RBBP7 mRNA extracted from two independent HGPS cell lines (\#1 and \#2) and a passage age-matched control cell line. The absolute values of RBBP4 and RBBP7 mRNA expression were normalized to the housekeeping gene Cyclophilin A. Values represent averages \pm S.D. from three experiments. (B) Immunofluorescence staining of control and HGPS primary dermal fibroblasts with the indicated antibodies. (C) Western blot of cell lysates prepared from control and HGPS dermal fibroblasts. (D) Rescue of RBBP4 protein levels upon correction of aberrant lamin A splicing in HGPS cells. Immunofluorescence staining with the indicated antibodies on HGPS primary dermal fibroblasts treated with either a sequencespecific morpholino oligonucleotide against the HGPS pathogenic mutation (EXO11) or a scrambled sequence control as previously described ${ }^{3}$. (E) Reduction of RBBP4 protein levels in HGPS patient cells expressing progerin. Immunofluorescence staining with the indicated antibodies on HGPS primary dermal fibroblasts. Scale bars: $10 \mu \mathrm{m}$. Arrowheads indicate cells with reduced levels of heterochromatin proteins. 
A

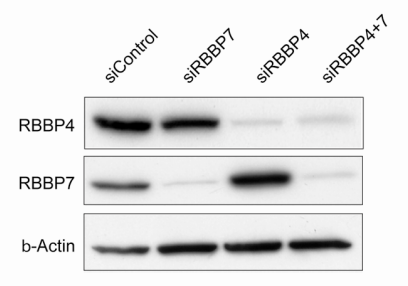

C

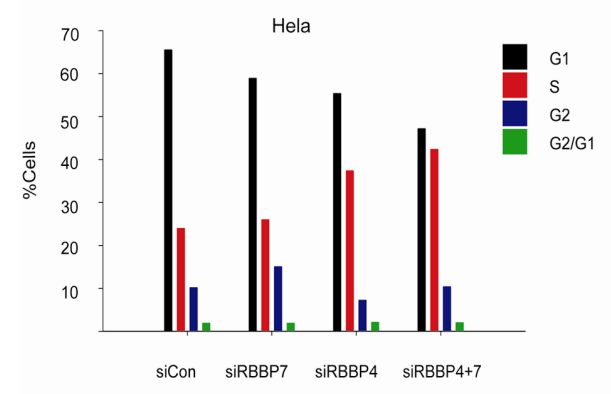

$E$
B

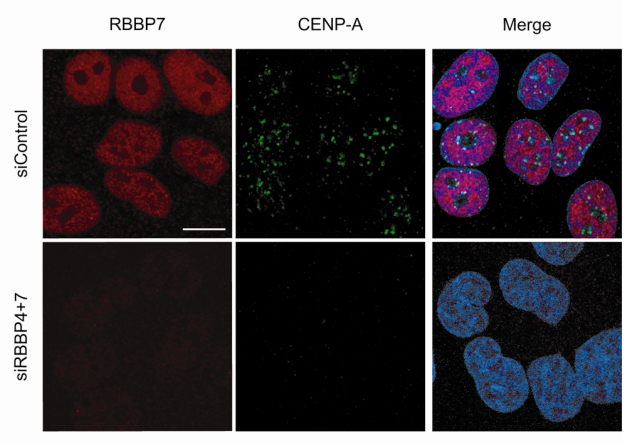

D

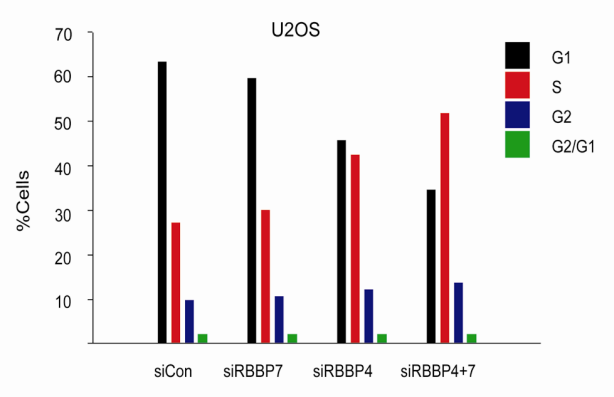

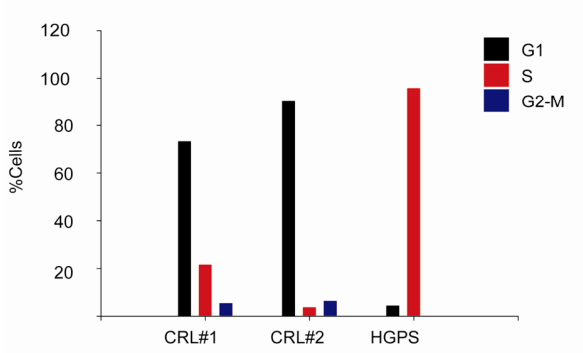

Figure S3. siRNA-mediated silencing of RBBP4 and RBBP7 induces accumulation of cells in S-phase. (A) Western blot of cell lysates prepared from Hela cells treated for $72 \mathrm{hrs}$ with the indicated combinations of siRNA oligos against RBBP4 and RBBP7. (B) Immunofluorescence staining with the indicated antibodies on Hela cells fixed $144 \mathrm{hrs}$ after oligo siRNA transfection. (C, D) Relative percentages of siRNA-treated Hela (C) or U2OS (D) cells in a specific phase of the cell-cycle $144 \mathrm{hrs}$ after oligo siRNA transfection. (D) Relative percentages of primary dermal fibroblasts in the different phases of the cell-cycle. Cells from two unaffected controls (\#1 and \#2) and a HGPS patient were passage-matched. Scale bar: $10 \mu \mathrm{m}$. 
A

H3K9me3 foci

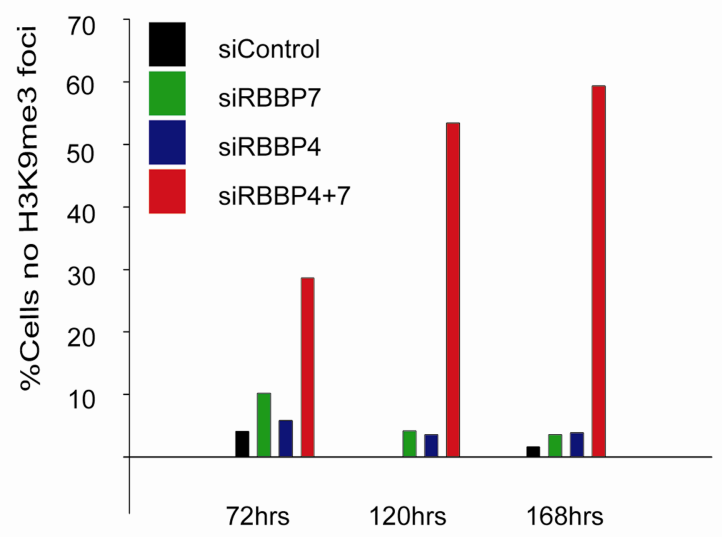

B

$$
p-H 2 A X
$$

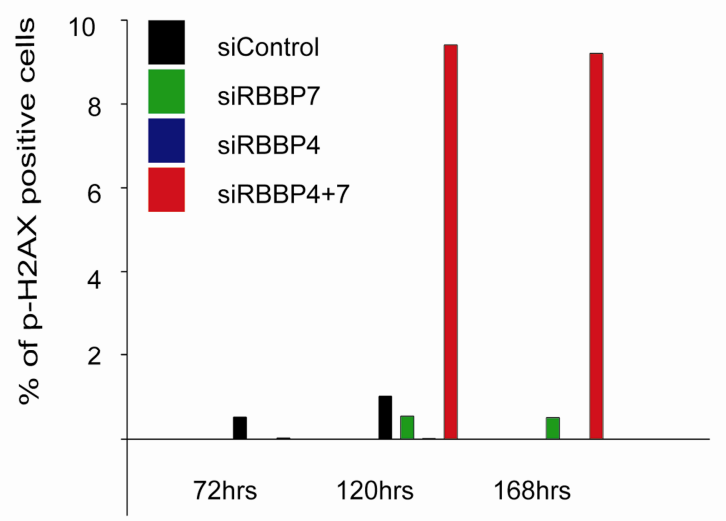

Figure S4. Kinetics of heterochromatin defects and DNA damage upon silencing of RBBP4 and RBBP7. Quantitation of the percentage of Hela cells that either exhibit loss of pericentromeric $\mathrm{H} 3 \mathrm{~K} 9 \mathrm{me} 3$ foci (A) or that were scored positive for phospho- $\mathrm{H} 2 \mathrm{AX}$ staining (more than 8 foci per cell) (B) at different time points after the transfection of the indicated combinations of siRNA oligonucleotides. $\mathrm{N}>200$. 
A
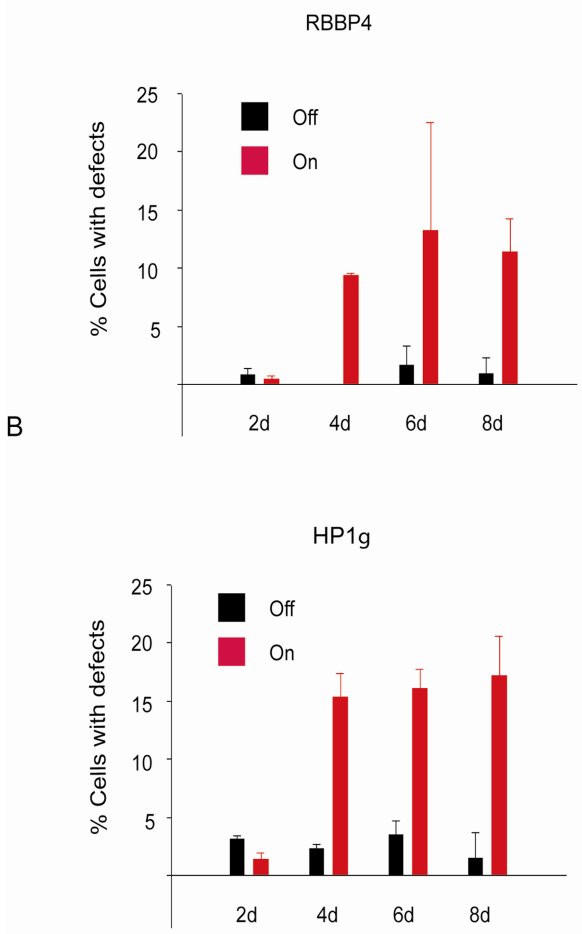

C

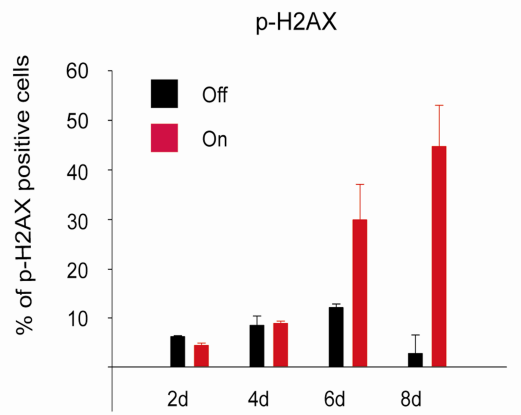

Figure S5. Kinetics of heterochromatin defects and DNA damage upon GFP-progerin expression. Quantitative single-cell analysis of fluorescence intensity signals at different time points (2-8 days) after the induction of GFP-progerin expression in normal immortalized human fibroblasts. Quantitation of the percentage of cells that either exhibit loss of RBBP4 (A) or HP1 $\gamma$ (B). (C) Quantitation of the percentage of cells that were scored positive for phospho-H2AX staining (more than 8 foci per cell) $\mathrm{N}>200$; values represent averages \pm S.D from three experiments 
A

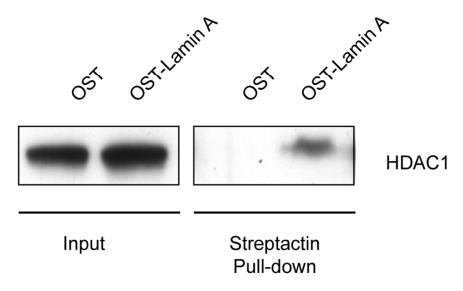

B

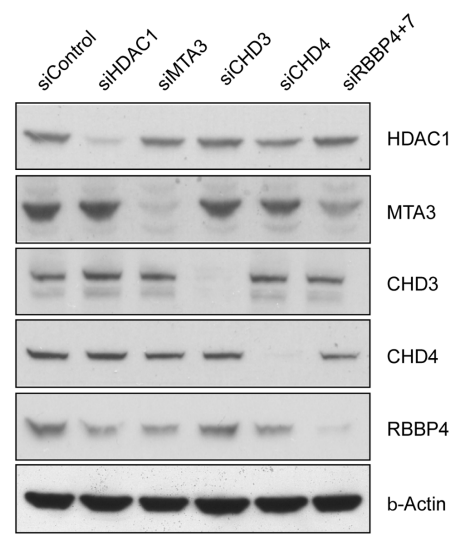

C

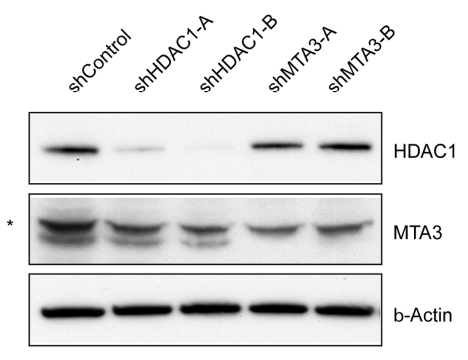

D

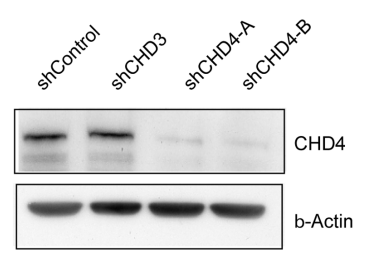

Figure S6. In vivo interaction of lamin A with HDAC1 and silencing of single NURD subunits in Hela cells and human primary fibroblasts.

(A) Crosslinked protein lysates were prepared from U2OS cells expressing One-Strep (OST)-tagged lamin $A$ and then incubated with Streptactin beads. Input and pulled down material was probed by Western blot with antibodies against endogenous HDAC1. (B) Western blots of Hela cells 72 hrs post-transfection with siRNA targeting different subunits of the NURD complex. (C) and (D) Western blot of cell lysates obtained from WI-38 human primary fibroblasts infected with lentiviral vectors expressing short hairpin RNA sequences against the indicated genes. The asterisk indicates a cross-reacting band in these lysates. 


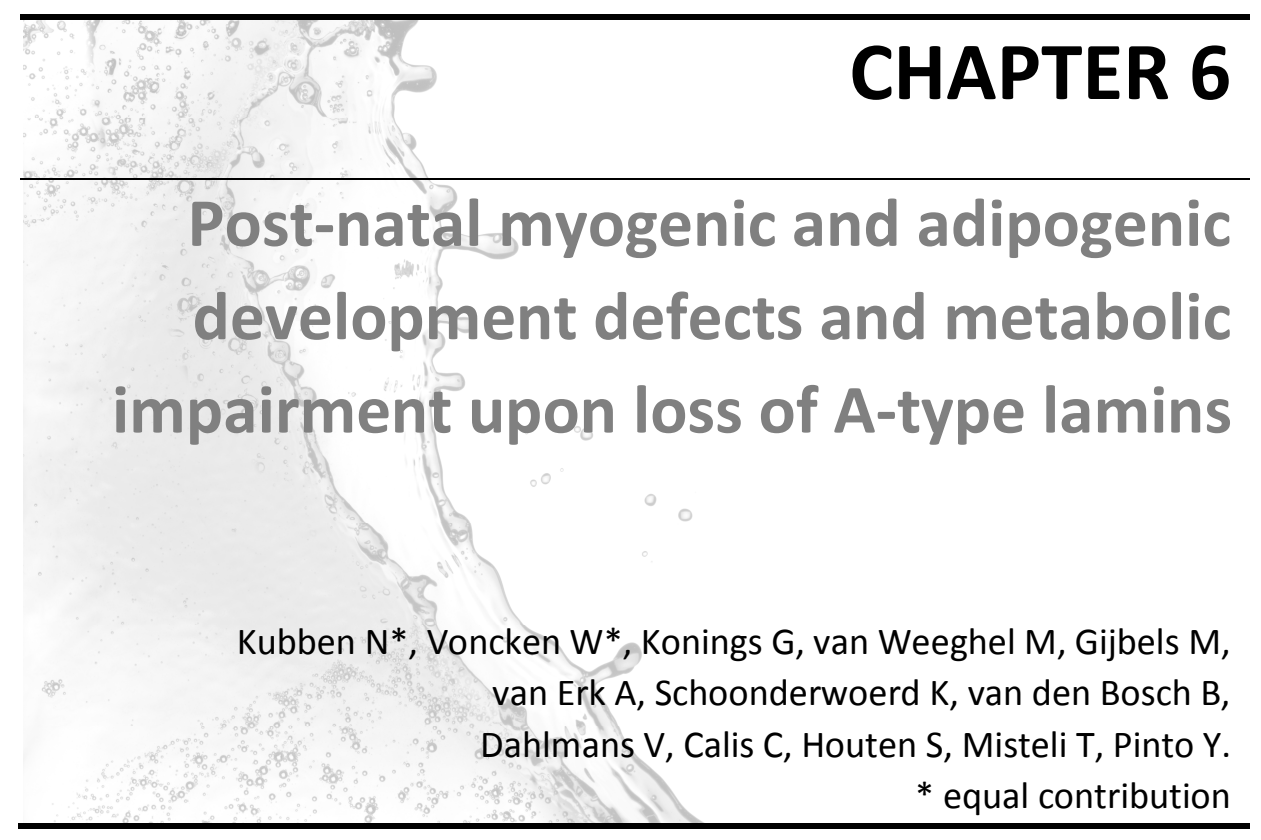




\section{Abstract}

A-type lamins are a major component of the nuclear lamina. Their expression is developmentally regulated and is absent during embryonic development until the onset of tissue differentiation and maturation. Mutations in the LMNA gene, which encodes the A-type lamins $A$ and $C$, cause a set of phenotypically diverse diseases collectively called laminopathies. While adult $L M N A$ null mice show various symptoms typically associated with laminopathies, the effect of loss of lamin $\mathrm{A} / \mathrm{C}$ on early post-natal development is poorly understood. Here we developed a novel LMNA null mouse $\left(L M N A^{G T-/}\right)$ based on genetrap technology and analyzed its early post-natal development. We detect $L M N A$ promoter activity in heart, liver, intestine and somites during early embryonic development. Loss of A-type lamins results in severe growth retardation and developmental defects of the heart, including impaired myocyte hypertrophy without functional cardiac defects, skeletal muscle hypotrophy, decreased amounts of subcutaneous adipose tissue and impaired ex vivo adipogenic differentiation. These defects cause death at 2 to 3 weeks post partum associated with muscle weakness and metabolic complications, but without the occurrence of dilated cardiomyopathy or an obvious progeroid phenotype. Our results indicate that defective early post-natal development critically contributes to the disease phenotypes in adult laminopathies. 


\section{Introduction}

Nuclear lamins are intermediate filament proteins mainly localized in the nuclear lamina, a protein meshwork lining the nucleoplasmic face of the inner nuclear membrane. Lamins are classified as A-type or B-type. The A-type lamins consist of the three $L M N A$ splicing isoforms lamin $A, A \Delta 10$ and $C$. B-type lamins are encoded by the $L M N B 1$ and $B 2$ genes. Whereas B-type lamins are expressed in all mammalian cells throughout development, A-type lamin expression is developmentally regulated. Lamin $A / C$ expression commences in the zygote but is lost at the morula stage ${ }^{1-3}$. It is only at the beginning of tissue differentiation, in mouse at day E12.0, that lamin $A / C$ expression restarts in myoblasts. In many other tissues, lamin $A / C$ was reported to be absent until well after birth 4-5. The notion of lamin $A / C$ being expressed upon tissue differentiation is further strengthened by its absence in embryonic stem cells and its reexpression after loss of pluripotency markers upon neuronal or cardiac myocyte differentiation ${ }^{6}$. Similarly, embryonic carcinoma cells are devoid of lamin $A / C$ in contrast to differentiated derivatives ${ }^{3,7}$. Loss of lamin $A / C$ results in increased proliferation due to hyperphosphorylation of the retinoblastoma protein ${ }^{8}$ and correlates with poor histological differentiation and prognosis in primary gastric carcinomas ${ }^{9}$.

Mutations in A-type lamins cause a group of phenotypically diverse diseases, collectively called laminopathies. They include several types of muscular dystrophies, lipodystropies, cardiomyopahties, neurological disorders and premature aging syndromes ${ }^{10}$. Several of the LMNA mutations affect cell differentiation. Mutations that lead to Dunnigan familial partial lipodystrphy (FPLD) interfere with the binding of lamin A to the adipocyte differentiation factor sterol element binding protein 1 (SREBP1) and impair adipocyte differentiation ${ }^{11}$. In the premature aging disease Hutchinson-Gilford Progeria Syndrome (HGPS), the diseasecausing lamin A mutant isoform progerin inhibits adipogenic differentiation and favors osteogenic differentiation of human mesenchymal stem cells via increased Notch signalling ${ }^{12}$. To study the 
role of lamin $\mathrm{A} / \mathrm{C}$ in skeletal, cardiac and adipose tissue and to probe how $L M N A$ mutations affect the functioning of these tissues in laminopathies, several lamin $A / C$ mouse models have been created ${ }^{13}$. Tissue defects in these models mostly become manifest in young adult mice and are therefore mainly studied in weaned mice. As a consequence, little is known about the effect of loss of lamin A/C on early post-natal development or the contribution of defective development to laminopathies.

In this study we set out to probe the effects of loss of lamin A/C on early post-natal development as this is the period in which most tissue differentiation and maturation occurs. To this end, we created a novel $\angle M N A^{\mathrm{GT} / \mathrm{-}}$ mouse model which combines loss of $L M N A$ function with LMNA-controlled reporter gene expression. We demonstrate early activity of the LMNA promoter at day E11 during embryonic development in heart, liver, intestine and somites. Loss of lamin A/C results in growth retardation at 2 weeks post partum, with impaired post-natal hypertrophy of cardiac myocytes, skeletal muscle hypotrophy, decreased subcutaneous adipose tissue deposits, decreased adipogenic differentiation of MEFs and metabolic derangements. Ultimately these tissue differentiation and maturation defects are lethal before mice are weaned. These results demonstrate that lamin A/C is crucial in the early post-natal period and they provide novel insights into the physiological function of A-type lamins in the context of the developing animal.

\section{Materials \& Methods}

\section{Targeting of the LMNA gene}

LMNA ${ }^{\text {Gt(FHCRC-GT-S7-1F1)Sor }}$ AK7.1 ES cells (strain of origin 129S4/SvJaeSor) containing a ROSAFARY Genetrap (GT) vector in the LMNA gene were obtained from the International Genetrap Consortium (www.genetrap.org). Chimaeric mice were created by ES cell injection into $\mathrm{C} 57 \mathrm{BI} / 6$ recipient blastocysts at the Transgenesis and gene 
Targeting Unit Maastricht (Maastricht, The Netherlands). Resulting chimaeric males were bred to wildtype (WT) C57BI/6 mice. Resulting heterozygous $L M N A^{G T}\left(L M N A^{G T+/-}\right)$ mice were interbred to achieve homozygocity $\left(L M N A^{G T-/}\right)$. For genotyping, PCR-primer pairs $\mathrm{FW}$ (forward) 5'GACGGGTTGTTACTCGCTCAC3' and Rev (reverse) 5'CAGGTCAAATTCAGACGGCAAA3' were used to selectively amplify GT; $\mathrm{FW}$ 5'ACCATCTCCCCAGCCCTTAG3' and

Rev 5'CAACATTCCTGATTCTTTCTGC3' to detect the endogenous LMNA gene. All studies involving animal experiments were approved by the Animal Care and Usage Committee of Maastricht University (Maastricht, The Netherlands) and the National Institutes of Health (NIH, Bethesda, USA) and were performed in accordance with regulations formulated in Dutch and US law on care and use of experimental animals.

\section{Histology, Immunohistochemisty and Transmission Electron Microscopy (TEM)}

$\angle M N A^{G T}$ mice were sacrificed by decapitation or cervical dislocation. Blood was removed from the vascular system by perfusing of $5 \mathrm{ml}$ physiological buffered saline via the left ventricle (LV) of the heart; muscle relaxation was induced by a consecutive injection with $100 \mu \mathrm{l}$ of a $0.1 \mathrm{~N}$ cadmium chloride solution. Tissues for histological analysis and immunohistochemistry were fixed for 4-6 hours in $4 \%$ paraformaldehyde, washed three times 5 minutes in PBS, stored overnight in $70 \%$ ethanol, embedded in paraffin and sectioned at 4 microns. Tissues sections were stained with haematoxylin eosin (H\&E) and Picro Sirius Red (SR) as described before ${ }^{14}$. H\&E staining was used for quantification of cardiac- and skeletal-myocyte cross sectional areas. Impaired post-natal hypertrophy was quantified by cardiac myocyte cross-sectional area (CSA) counts in LV myocytes opposite or adjacent to the septum. Tissues for RNA isolation were snap frozen in liquid nitrogen. Tissues for TEM analysis were fixed in $2.5 \%$ glutaraldehyde and further analysis was performed as previously described ${ }^{15}$. Whole-mount embryos were $\beta$-galactosidase stained as described ${ }^{16}$. 


\section{RNA isolation and microarray analysis}

RNA was isolated from mouse embryonic fibroblasts (MEF) and LV of $\angle M N A^{G T}$ mice using the RNAeasy minikit (Qiagen, Hilden, Germany). For whole transcriptome analyses, RNA from LV's of 5 and 13 days old WT and $L M N A^{\mathrm{GT}-\mathrm{-}}$ mice ( $\mathrm{N}=2$ for each genotype and age) was isolated and hybridized to Nugo Mouse Affymetrix expression arrays. Intensity values after hybridization were normalized to the median signal intensity of the array. Enrichment of deregulated genes in specified biological pathways was tested using GENMAPP 2.1 software. For expression analysis by PCR cDNA was synthesized with the iScript ${ }^{\mathrm{TM}}$ CDNA synthesis kit (BioRad, Hercules, USA). SYBR Green real-time quantitative PCR analysis was performed with primers for ANF (Fw 5'ATTGACAGGATTGGAGCCCAGAGT3', Rev 5'TGACACACCACAAGGGCTTAGGAT3'), BNP (FW 5'GTTTGGGCTGTAACGCACTGA3', Rev 5'GAAAGAGACCCAGGCAGAGTCA3'), Ki67 (Fw 5'TCAACAGCtGGtATGCCTAACAG3', Rev 5'TTCCAGTGGTCAAAGAGTCATTAGC3'), PCNA (FW 5'AGGGTTGGTAGTTGTCGCTGTAG3', Rev 5'GGTCCCCCGATTCACGAT3'), GAPDH (FW 5' GGTGGACCTCATGGCCTACA3', Rev 5'CTCTCTTGCTCAGTGTCCTTGCT3') and HPRT (FW 5'GCGTCGTGATTAGCGATGATGAAC3', Rev 5'CCTCCCATCTCCTTCATGACATCT3').

\section{Echocardiography and electrocardiogram (ECG) analysis}

WT, $L M N A^{\mathrm{GT}+/}$ and $L M N A^{\mathrm{GT}-/}$ cardiac functioning was monitored by serial echocardiography and ECG analysis at days 10, 13 and 17 post partum (PP10, PP13 and PP17). ECG analysis was performed under $2 \%$ isoflurane sedation; $\mathrm{P}$ wave, QRS and QT time were assessed using IDEEQ v1.7 software (Instrument Development Engineering \& Evaluation, Maastricht University, The Netherlands). Echocardiography was performed uder similar sedative conditions, with a $30 \mathrm{MHz}$ transducer on a Vevo $770^{\mathrm{TM}}$ high-resolution in vivo micro-imaging system (VisualSonics B.V., Amsterdam, The Netherlands). LV parameters were obtained from M-mode recordings in the short axis-view. Fractional shortening (FS) was calculated using the the formula: FS $=100 x($ (Left Ventricle Inner Diameter in diastole - Left Ventricle Inner Diameter in systole)/Left Ventricle Inner diameter in diastole). To study the effects of $\alpha$ - and $\beta$-adrenergic receptors antagonists on $L M N A^{\mathrm{GT} /-}$ cardiac 
performance, labetalol was administered twice daily by subcutaneous interscapular injections at a dose of $10 \mathrm{mg} / \mathrm{kg}$ from day PP10 onward; ECG analysis was performed as indicated above at day PP13. To study the effects of LMNA heterozygosity on cardiac hypertrophy, echocardiography was performed as indicated above on 60-70 weeks old $\angle M N A^{\mathrm{GT}+/}$ mice and WT controls. Furthermore 16-18 weeks old WT and $L M N A^{\mathrm{GT}+/}$ mice were infused subcutaneously with $1.5 \mu \mathrm{g} / \mathrm{g} /$ day angiotensin II for 4 weeks by an osmotic minipump (Alzet osmotic minipumps, Cupertino, CA). Echocardiograms were made at 2 and 4 weeks after the start of the angiotensin II treatment.

\section{Funtional assessment of skeletal muscles.}

Total quadriceps muscle weight were normalized to tibia length for all genotypes. Skeletal muscle strength was assessed by quantifying the capacity to hang upside down on a grid with use of both fore- and hind limbs. Time in seconds indicates the time from placing the raster upside down until the mouse dropped from the grid. Measurements on each genotype were performed in triplicate $(n=4$, PP15).

\section{Adipocyte differentiation assay}

MEFs were isolated from E12.0 LMNA ${ }^{\mathrm{GT}-\mathrm{-}_{-}}, L M N A^{\mathrm{GT+}+-}$ and WT embryos by digesting skin isolates for $20 \mathrm{~min}$ in $3 \mathrm{ml} 0.05 \%$ trypsin at $37^{\circ} \mathrm{C}$ during which the solution was resuspended every 10 minutes. This was followed by addition of an equal volume of fresh trypsin solution and a 20 minute incubation step; this was repeated once and incubated for 5 additional minutes, after which a cell pellet was obtained by centrifugation and plated in Dulbecco's modified eagle medium (11360070, Invitrogen, San Diego, USA) with $2 \mathrm{mM}$ L-glutamine, $10 \mathrm{mM}$ nonessential amino acids (NEAA), $110 \mathrm{mg} / \mathrm{l}$ pyruvate, 10\% fetal bovine serum (FBS) and antibiotics. Passage 2 (P2) cells were seeded at $50 \%$ confluency in 6-wells plates for adipogenic differentiation and grown to confluency for 3 days. The medium was replaced and cells were left for 3 days on induction medium: hMSC basal medium (PT-3238, Lonza, Basel, Switzerland) with $2 \mathrm{mM}$ L-glutamine, $10 \mathrm{mM}$ NEAA, $110 \mathrm{mg} / \mathrm{l}$ pyruvate, 
$10 \% \mathrm{FBS}$, antibiotics, $10 \mu \mathrm{g} / \mathrm{ml}$ insulin, $0.5 \mathrm{mM}$ 3-isobutyl-I-methylxantine (IBMX) and $1 \mu \mathrm{M}$ dexamethasone. After 3 days the medium was replaced by maintenance medium (similar to induction medium with omission of IBMX and dexamethasone) and left for 2 days. This 5 day cycle of induction and maintenance was repeated three times. Oil Red $\mathrm{O}$ staining and quantification after each cycle (6, 10 and 16 days post induction) were performed in triplicate as previously described ${ }^{12}$.

\section{Metabolic characterization}

To asses core body temperature, mice received an inter-intestinal temperature probe through a small abdominal incision directly after applying isoflurane anesthesia. Core body temperature was measured as soon as it reached a plateau phase (i.e. before ambient temperature decreased body temperatures). Subcutaneous adipose tissue deposits were determined by incising the skin along the saggital axis, collecting and weighing subcutaneous adipose tissue in between the left fore- and hind leg. Subcutaneous adipose tissue weights were standardized by tibia length. For blood chemistry measurements, WT, $L M N A^{G T+/-}$ and $L M N A^{\mathrm{GT}-/}$ mice were intraperitoneally injected with $50 \mu \mathrm{l}$ of a 500 Units/ml heparin solution as an anticoagulant before decapitation and collection of blood. For glucose, lactate and betahydroxybutyrate analysis the collected blood was mixed with equal amounts of $1 \mathrm{M}$ perchloric acid and centrifuged to obtain a supernatant fraction for further analysis ${ }^{17}$. The acylcarnitine spectrum was determined by mass spectrometry on regular blood plasma as described ${ }^{18}$. Urea, creatinine and creatinine kinase were determined on EDTA anticoagulated blood. For mitochondrial functioning LV samples were snap frozen in liquid nitrogen and used to determine catalytic activity of citrate synthase and individual oxidative phosphorylation complexes as described ${ }^{19}$. Mitochondrial DNA copy numbers were assessed in LV cardiac muscle and quadriceps skeletal muscle ${ }^{19}$. 


\section{Statistical analysis}

Data are represented as average \pm SD. The data for each study group were compared using 2-way ANOVA or student's t-test where appropriate. Analyses were performed using the statistical package SPSS 11.0. $\mathrm{P}<0.05$ was considered to be statistically significant.

\section{Results}

\section{Generation of a Lamin ${ }^{\text {GT-/ }}$ mouse}

We created $L M N A$ null mice by interruption of the endogenous lamin $\mathrm{A} / \mathrm{C}$ locus by a promoter-trap construct, which introduces an in-frame $\angle M N A-\beta g e o$ fusion allele. The construct was inserted in second $L M N A$ intron (Figure 1A). Lamin $\mathrm{A} / \mathrm{C}$ transcripts (Figure $1 \mathrm{~B}$ ) and protein were undetectable in $L M N A^{\mathrm{GT}-/}$ animals 15 days post partum. As expected, $\angle M N A^{\mathrm{GT}+/}$ mice expressed approximately half the amount of lamin $\mathrm{A}$ and C mRNA (Figure 1B) and protein compared to WT controls. RT-PCR analysis confirmed the absence of randomly integrated GT vector sequences elsewhere in the genome, as well as the absence of alternative $L M N A$ splicing products (data not shown).

The $L M N A-\beta$-geo fusion protein is encoded by the first $L M N A$ exon followed by $\beta$-galactosidase-neomycin cDNA (Figure $1 \mathrm{~A}$ ), which allows for direct visualization of LMNA promoter activity in $L M N A^{G T+/-}$ and $\angle M N A^{\mathrm{GT}-/}$ whole mounts and tissue sections. At E8.0 and E9.0, only $\angle M N A^{\mathrm{GT}+/}$ placental tissue showed LMNA promoter activity (Figure $1 \mathrm{C}$ ). At E11.0 the LMNA promoter was active in heart, liver, intestines and embryonic somites. Late in embryonic development (E18.0) LMNA promoter activity was observed macroscopically in all organs. Specificity of $\beta$-galactosidase signal was confirmed by staining adult $L M N A^{\mathrm{GT+}+\text { - }}$ mice, which showed absence of LMNA promoter activity in various cell types, as reported previously ${ }^{4}$ (data not shown). 
At birth, genotypes were present in normal Mendelian ratios and no obvious macroscopic differences were detected between WT, LMNA ${ }^{\mathrm{GT}+\text { - }}$ and $L M N A^{\mathrm{GT}-/-}$ mice. At PP7, onset of initial growth retardation was observed in $L M N A^{G T-/}$ mice, which was confirmed by decreased body weight (BW) (Figure 1D and E). LMNA ${ }^{\mathrm{GT}-/-}$ body weight peaked at PP13 and decreased slightly thereafter, leveling off at approximately half the BW of that of WT and $L M N A^{\mathrm{GT}+/-}$ at PP16 (Figure 1E). Complete loss of lamin $A / C$ due to insertion of the GT sequence was invariably lethal between PP16 and PP18 (Figure 1F). BW and survival curves of $L M N A^{\mathrm{GT+}+\text { - }}$ mice were comparable to those of WT siblings. Grooming behavior and foraging appeared normal for all genotypes, and were only diminished for $L M N A^{\mathrm{GT}-/}$ mice at days PP16-PP18. $L M N A^{\mathrm{GT}-/}$ autopsy at PP17 revealed an increased amount of intra-intestinal gas, but did not reveal obvious differences in food content or Gl tract histology. Pathological examination at PP15 revealed heart, skeletal muscle myocytes and adipose tissue abnormalities. LMNA ${ }^{\mathrm{GT}-/}$ mice did not show overt abnormalities in stomach, intestines, liver, kidneys, spleen, thymus, thyroid, lung, bladder, pancreas and brain. No obvious indications of abnormal bone or dentation were observed (data not shown), in contrast to reports on other LMNA mouse models ${ }^{13}$. Hence, we conclude that loss of lamin $A / C$ leads to defects in early post-natal development and ultimately death.

\section{Transcriptome analysis}

To assess how loss of lamin A/C affects tissue differentiation and maturation, microarray expression analysis was performed on left ventricle (LV) cardiac tissue at PP5 and PP13. At PP5 expression of 671 genes changed more than 1.2 fold (199 up, 472 down) in $L M N A^{G T-/-}$ mice compared to WT siblings (Figure $2 \mathrm{~A}$ and $\mathrm{B}$ ). 28 of those genes were still misregulated at PP13, 3 of which in similar and 25 in opposite direction. In addition, 359 genes were more than 1.2 fold deregulated at PP13 (194 up, 165 down) while normally expressed at PP5 (Figure 2A and B). Genmapp analysis indicates that the deregulated genes are significantly 


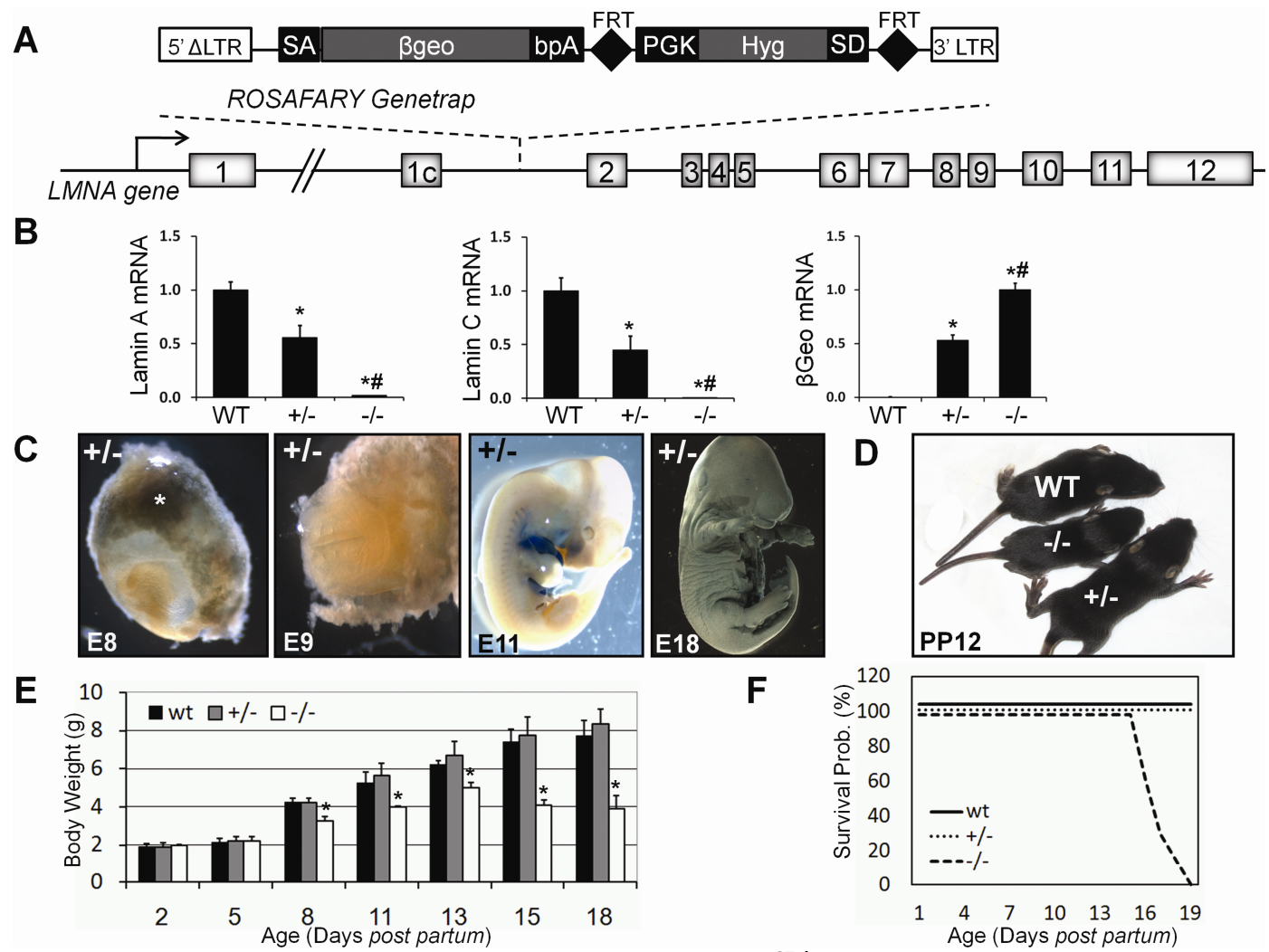

Figure 1. General phenotypical characterization of the $L M N A^{G T-\%}$ murine model. (A) Overview of the ROSAFARY genetrap (GT) sequence insertion in the LMNA gene. The GT consists of a splice acceptor (SA) containing Bgeo-reporter with a polyA signal (bpA) and a PGK promoter driven hygromycin antibiotics resistance cassette surrounded by FRT sites and harbouring a splice donor (SD) site. $5^{\prime}$ and $3^{\prime}$ ends of the GT sequence are demarcated by long terminal repeats (LTR). (B) Relative mRNA expression levels for full length lamin $\mathrm{A}, \mathrm{C}$ and the $L M N A$ - $\beta$ geo splicing product. Expession levels are normalized to HPRT mRNA levels. (C) $\angle M N A$ promoter activity is visualized by $\beta$-galactosidase staining in $L M N A^{G T+/-}$ embryo's (E8.0, E9.0, E11.0 and E18.0). $L M N A^{G T+/-}$ maternal placental tissue is indicated by an asterisk. (D) Macroscopical view of WT, $L M N A^{\mathrm{GT}+-}$ and $L M N A^{\mathrm{GT} /-}$ siblings 12 days post partum (PP12). (E) Body weight over time graph (PP2-PP18). Asterisks indicate a significant difference for $L M N A^{\mathrm{GT}-/-}$ opposed to $L M N A^{\mathrm{GT}+\text { - }^{-}}$and WT littermates $(\mathrm{N}=10$, $\mathrm{P}<0.05)$. (F) Survival curves for all three genotypes during the first 3 weeks post partum. 
$(\mathrm{P}<0.05)$ over-represented in fatty acid beta oxidation (PP5), striated muscle contraction (PP5), electron transport chain (PP13), adipogenesis (PP13) and hypertrophy (PP13). The most highly deregulated genes included Mybcp2, My6, Tnni1 (striated muscle contraction); Hdac9, Gata4, Atf3 (hypertrophy); Dci, Slc25a20 (fatty acid beta oxidation); Cebpd, Nrip, DIk (adipogenesis) and ATP5g2, Cox8a, Cox8b (electron transport chain) (Figure $2 \mathrm{C}$ ). These results indicate that loss of lamin $\mathrm{A} / \mathrm{C}$ resulted in myogenic, adipogenic and metabolic defects during early post-natal stages of development.

\section{Cardiac morphology phenotype}

In keeping with transcriptome analysis, histological examination revealed that cardiac myocytes were smaller in the $L V$ of $L M N A^{\mathrm{GT}-/-}$ mice compared to WT controls (PP13; Figure 3A). We examined cardiac hypertrophy by weighing LV as a function of time post partum. LV weight (LVW; normalized to tibia length) was decreased by $14 \%$ at PP11 and up to $47 \%$ at PP15 in $L M N A^{\text {GT-/ }}$ mice compared to WT age-matched controls (PP11 2.2 $\pm 0.2 \mathrm{mg} / \mathrm{mm} L M N A^{\mathrm{GT}-/-}$ vs. $2.6 \pm 0.2 \mathrm{mg} / \mathrm{mm}$ WT $\mathrm{p}<0.05$; PP15 $1.9 \pm 0.3 \mathrm{mg} / \mathrm{mm} L M N A^{\mathrm{GT} /-}$ vs. $3.6 \pm 0.3 \mathrm{mg} / \mathrm{mm}$ WT $\mathrm{p}<0.01$ ) (Figure $3 \mathrm{~B}$ ). Post-natal hypertrophy was significantly impaired at PP11 in homozygous GT animals: cross-sectional aera (CSA) counts were nearly 2-fold reduced $\left(116 \pm 17 \mu \mathrm{m}^{2} L M N A^{\mathrm{GT}-/-}\right.$ vs. $220 \pm 34 \mu \mathrm{m}^{2}$ WT; Figure $3 C$ $\mathrm{p}<0.01$ ), and reached a substantially lower maximum of $153 \pm 17 \mu \mathrm{m}^{2}$ in $L M N A^{G T-/}$ mice vs $380 \pm 8 \mu m^{2}$ in WT siblings $(p<0.001)$. Real-time quantitative PCR analysis showed comparable expression levels of the proliferative markers Ki67 and PCNA in the LV of all genotypes in early post-natal development and a general decrease in levels after birth ( $>0.05$; Figure $3 C$ ). The cardiac hypertrophy markers $B N P$ and ANF showed 10- to 100-fold upregulation in $L M N A^{\mathrm{GT} / \mathrm{-}}$ mice, at PP11 and PP13 and onwards, respectively ( $p<0.05$; Figure $3 D$ ). No cardiac myocyte degenerative areas, fibrosis or obvious abnormalities in interstitial spaces and Z-disc organization were observed (data not shown). These data show that A-type lamins are essential for normal post-natal cardiac hypertrophy. 
A
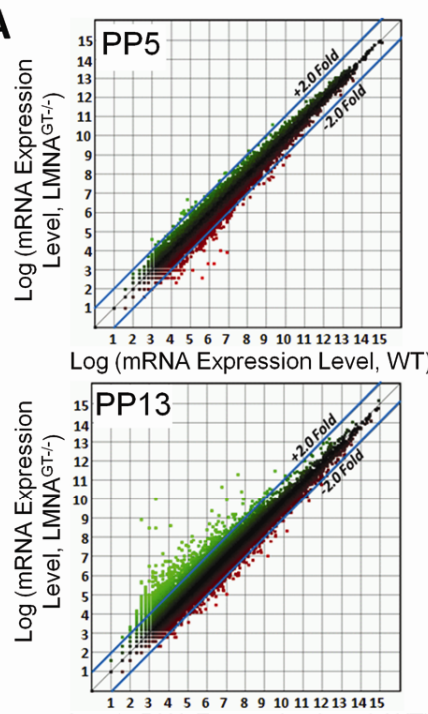

Log (mRNA Expression Level, WT)

B

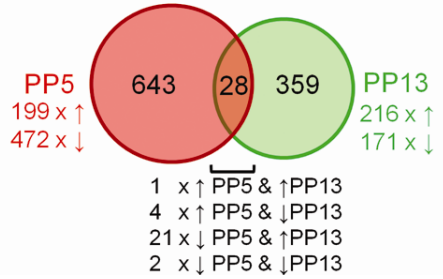

C

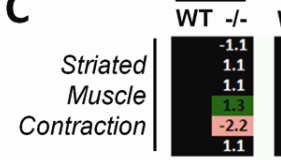

Hypertrophy $\mid \begin{array}{r}-2.3 \\ 1.3 \\ -1.2 \\ -1.8 \\ -1.1 \\ 1.5 \\ 1.2 \\ 1.7 \\ 1.7\end{array}$
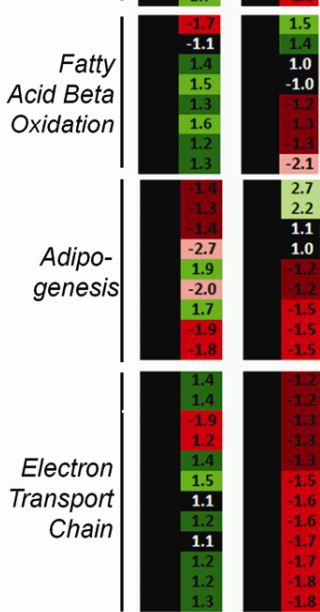

$\begin{array}{r}\hline-1.2 \\ -1.2 \\ -1.3 \\ -1.3 \\ -1.3 \\ -1.5 \\ -1.6 \\ -1.6 \\ -1.7 \\ -1.7 \\ -1.8 \\ -1.8 \\ \hline\end{array}$

$\underline{\text { PP13 }}$
8.0 Actin, gamma, cytoplasmic 1

3 Pdz and lim domain 3

Myosin, light polypeptide 6 (Myl6)

Troponin I, skeletal, slow 1 (Tnni1)

Myosin binding protein C, fast-type (Mybcp2)

Myosin, light polypeptide 9 , regulatory (MYl9)

Activating transcription factor 3 (Atf3)

Myocardin (Myocd)

Myocardin (Myocd)

Heart and neural crest derivatives expressed transcript 2 (Hand2)

Interferon-related developmental regulator 1 (Ifrd1)

Myosin, heavy polypeptide 6, cardiac muscle, alpha (Myh6)

Cyclin I (Ccnl)

Eukaryotic translation initiation factor $4 \mathrm{E}$ binding protein 1 (Eif4ebp1) Gata binding protein 4

Acyl-CoA synthetase long-chain family member 4(Acs/4)

Solute carrier family 25 , member 20 (SIc25a20)

Acyl-coa thioesterase 2 (Acot2)

Hydroxyacyl-Coenzyme A dehydrogenase

Dodecenoyl-Coenzyme A delta isomerase (Dci)

Carnitine palmitoyltransferase $\mathbf{2}$ (Cpt2)

Hydroxysteroid (17-beta) dehydrogenase 4 (hsd17 b4)

Sterol carrier protein 2

CCAAT/enhancer binding protein (C/EBP) (Cebpd)

2 Nuclear receptor interacting protein 1 (Nrip1)

1 Sortilin-related receptor, Idlr class a repeats-containing

Leptin receptor

Delta-like 1 homolog (Dlk1)

Necdin (Ndn)

GATA binding protein 4

Hexosaminidase b (Hexb)

Isopentenyl-diphosphate delta isomerase (Idi1)

Electron transferring flavoprotein, dehydrogenase (Etfdh) Cytochrome c oxidase, subunit VI a, polypeptide 2 (Cox6a2)

Thioredoxin-like 1 (Txnl1)

Cytochrome coxidase, subunit VIb polypeptide 1 (Cox6b1)

ATP synthase mitochondrial F1 complex, delta subunit (Atp5d)

Solute carrier family 25 (SIc25a13)

NADH dehydrogenase (ubiquinone) 1 alpha subcomplex, 7 (Ndufa7)

NADH dehydrogenase (ubiquinone) fe-s protein 8 (Ndufs8)

Cytochrome c oxidase, subunit VIIIb (Cox8b))

Cytochrome coxidase, subunit VIIla (Cox8a)

Expressed sequence AI256775 (AI256775)

ATP synthase mitochondrial F0 complex, subunit c, isoform 2 (Atp5g2)

$<-2.0$

Figure 2. Transcriptome analysis of the $L M N A^{G T-/-}$ mouse. (A) mRNA expression levels of left ventricle cardiac tissue were analyzed by microarrays at PP5 and PP13. Scatter plots indicate logarithmic expression levels of mRNA of $L M N A^{G T-/}$ mice vs. WT littermates at both time points. Twofold up- and down regulation borders are indicated by straight lines within the scatter plots. (B) A Venn diagram of over 1.2 fold up- or down-regulated genes in the $L M N A^{\mathrm{GT}-/}$ mice compared to WT siblings at PP5 and PP13. The number of genes and direction of change are indicated for all deregulated genes at PP5, PP13 and genes which are deregulated at both time points. (C) Heatmaps with mRNA expression levels of the most deregulated genes at PP5 and PP13 within the biological pathways striated muscle contraction, hypertrophy, fatty acid beta oxidation, adipogenesis and electron transport chain. WT expression levels for each gene were set at 1.0 (black color code). Relative differences in expression levels for $L M N A^{\mathrm{GT}-/}$ mice are indicated as fold change values and color coded. 
A

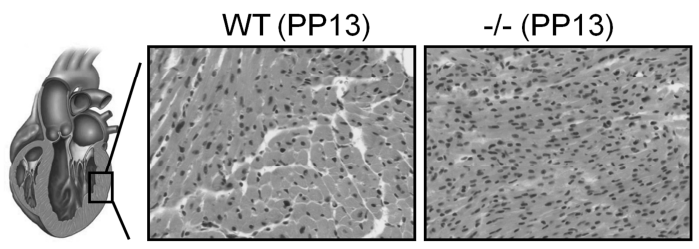

B

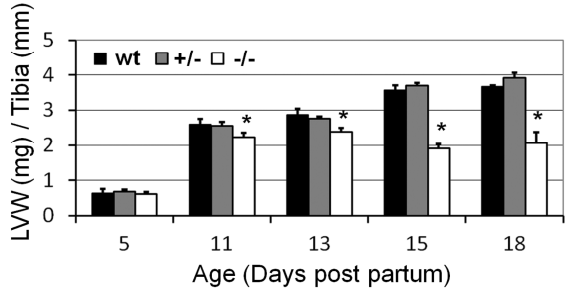

C

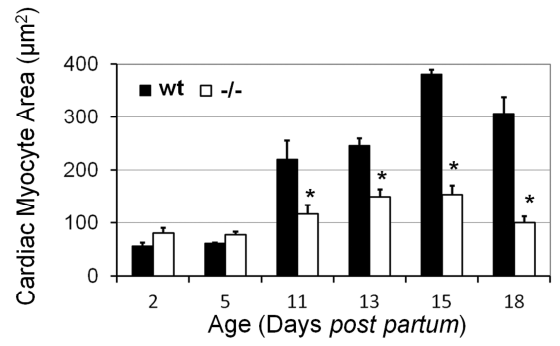

D
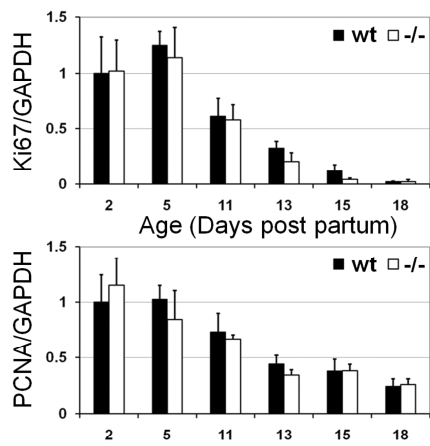

Age (Days post partum)

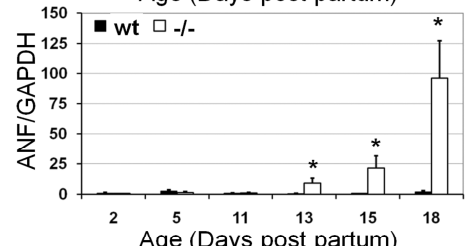

Age (Days post partum)

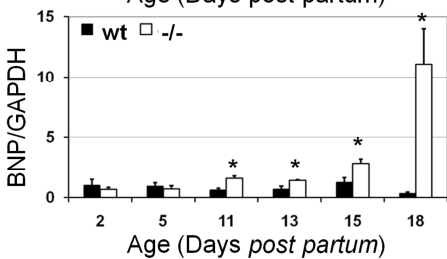

Figure 3. Post-natal cardiac hypertropy in the $L M N A^{\mathrm{GT}-/-}$ mouse. (A) Haematoxylin and eosin (H\&E) staining on left ventricle sections of WT and $L M N A^{\mathrm{GT}-/}$ mice (PP13). (B) Bar graph indicating left ventricle weight (LVW) standardized by tibia length over time (PP5-PP18) for all three genotypes. (C) Bar graph with cardiac myocytes cross sectional area over time (PP2-PP18) for $L M N A^{G T-/-}$ and WT sibblings. (D) mRNA levels of proliferative markers Ki67 and PCNA and hypertrophic markers $A N F$ and $B N P$, standardized for GAPDH. Asterisks in figure 3 indicate significant differences for $L M N A^{G T-/-}$ vs. both $L M N A^{G T+/-}$ and $W T$ values $(\mathrm{N}=5, \mathrm{P}<0.05)$.

\section{Cardiac function phenotype}

To determine whether decreased cardiac hypertrophy results in compromised cardiac function, $L M N A^{G T}$ mice were subjected to echocardiography and electrocardiography (ECG). Impaired post-natal hypertrophy was confirmed by lower relative $\mathrm{LVW}$ in $L M N A^{\mathrm{GT} /-}$ mice (PP17: $2.1 \pm 0.3 \mathrm{mg} / \mathrm{cm} L M N A^{\mathrm{GT}-/-}$ vs. $3.7 \pm 0.1 \mathrm{mg} / \mathrm{cm}$ WT $\mathrm{p}<0.01$; Table 1 ). Nevertheless, fractional shortening (FS), which measures the fraction of LV diastolic dimension lost in systole, as an indicator for LV contractility, 


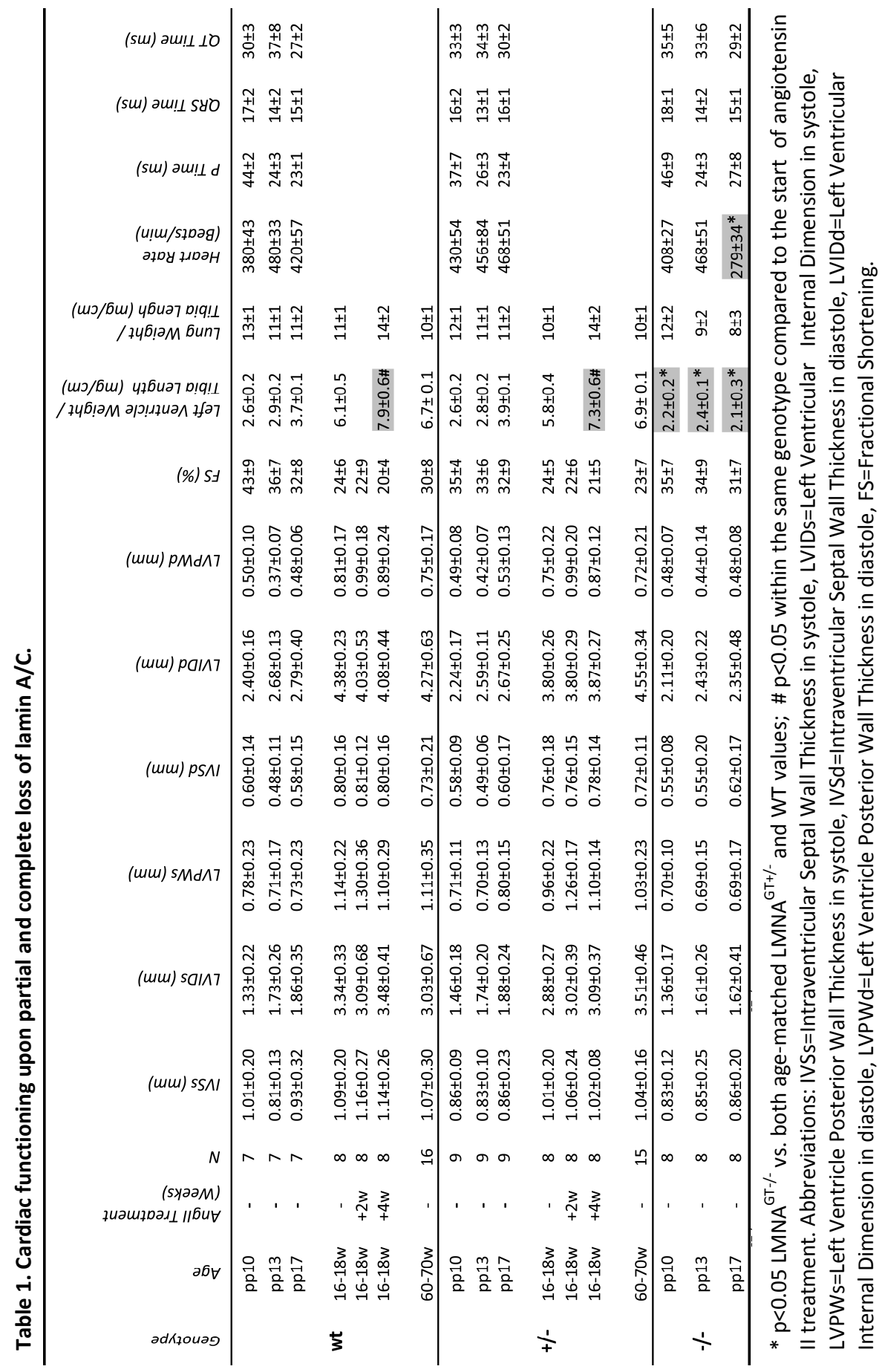


was comparable in all 3 genotypes at days PP10, PP13 and PP17 (PP17: $\left.31 \pm 7 \% L M N A^{\mathrm{GT}-/}, \quad 32 \pm 9 \% L M N A^{\mathrm{GT}+/}, 32 \pm 8 \% \mathrm{WT}\right)$. No signs of $\mathrm{LV}$ dilatation nor thinning of the LV wall were observed as all echocardiographic parameters appeared comparable for all genotypes (Table 1). ECG analysis, which captures electrical activity of the heart over time, did not reveal any differences in $\mathrm{P}$ wave duration (reflects atrial depolarization), QRS time (reflects ventricular depolarization), or QT time (prolonged QT time is a risk factor for ventricular tachyarrythmias and sudden death). ECG analysis did reveal a significantly lower heart rate in $L M N A^{G T-/}$ mice from day PP15 onwards (PP17: $279 \pm 34 \mathrm{bpm}$. $L M N A^{\mathrm{GT}-/}$ vs. $420 \pm 57 \mathrm{bpm}$. WT $\left.\mathrm{p}<0.05\right)$. Decreasing cardiac workload by lowering blood pressure and decreasing heart rate was reported to be beneficial in dilated cardiomyopathy, which is observed in various LMNA mouse models ${ }^{13,20}$. We therefore assessed whether treatment with the $\alpha$ - and $\beta$-adrenergic antagonist labetalol had beneficial effects in $L M N A^{\mathrm{GT}-/-}$ mice (Figure S1A). Labetalol increased QRS time in all genotypes $(\mathrm{p}<0.05)$. In contrast to $\mathrm{WT}$ and $L M N A^{\mathrm{GT+}+\text { - }}$ mice, labetalol treatment significantly increased $\mathrm{P}$ time (from $23.7 \pm 2.6 \mathrm{~ms}$ to $29.8 \pm 3.2 \mathrm{~ms} ; \mathrm{P}<0.05)$ and lowered heart rate $(396 \pm 51 \mathrm{bpm}$ to $232 \pm 20$ bpm; $\mathrm{p}<0.05$ ) in $L M N A^{\mathrm{GT}-/}$ mice (Figure S1B). However, treatment with labetalol lead to earlier post-natal death in $\angle M N A^{\mathrm{GT}-/}$ mice compared to $L M N A^{G T+/-}$ and WT siblings (Figure S1C).

To assess whether age-related physiological cardiac hypertrophy is subject to gene dosage effects 60-70 weeks old WT and $L M N A^{G T+/-}$ mice were subjected to echocardiography. Although the average FS was slightly decreased in $L M N A^{G T+/-}$ mice, this difference was not significant $\left(23 \pm 7 \% L M N A^{G T+/-}\right.$ vs. $30 \pm 8 \%$ WT $p>0.05$; Table 1). Other cardiac parameters did not differ significantly. Similarly, angiotensin II treatment induced comparable levels of hypertrophy under acute pathological conditions in 16-18 weeks old $\angle M N A^{\mathrm{GT+} /-}$ and WT mice, based on relative LVWs ( $L M N A^{G T+/-}$ from $5.8 \pm 0.4$ to $7.3 \pm 0.6 \mathrm{mg} / \mathrm{cm} \mathrm{p}<0.05$; WT from $6.1 \pm 0.5$ to $7.9 \pm 0.6 \mathrm{mg} / \mathrm{cm} \mathrm{p}<0.05)$. No signs of cardiac failure were observed as FS, other echocardiographic parameters and relative lung 
weight were all comparable ( $p>0.05$; Table 1$)$. Therefore, we conclude that partial loss of lamin A/C only slightly decreased LV weight without impairing the ability to respond structurally or functionally to increased load. Complete loss of lamin A/C impairs post-natal cardiac hypertrophy, lowers the heart rate moderately but does not cause any other direct functional abnormalities.

\section{Skeletal muscle phenotype}

Initial gross anatomical examination showed hunched posture and abnormal gait, characterized by splayed hind legs from day PP13 onwards in $L M N A^{G T-/}$ mice. Histological examination revealed smaller quadriceps skeletal myocytes at day PP15 (Figure 4A). Based on transcriptome analysis and these initial findings we further assessed post-natal skeletal muscle development. Pathological findings were confirmed by quantification of the CSA of skeletal myocytes and the total quadriceps muscle weight (normalized to tibia length). Quadriceps muscle in $L M N A^{\mathrm{GT}-/}$ mice showed hypotrophy at PP15 (relative quadriceps weight $5.0 \pm 0.5 \mathrm{mg} / \mathrm{mm} L M N A^{G T-/}$ vs. $7.1 \pm 0.6 \mathrm{mg} / \mathrm{mm}$ WT $\mathrm{p}<0.05)$ whereas they did not at PP9 $\left(3.8 \pm 0.95 \mathrm{mg} / \mathrm{mm} L M N A^{G T-/-} v s\right.$. $4.7 \pm 0.35 \mathrm{mg} / \mathrm{mm}$ WT $\mathrm{p}>0.05$; Figure $4 \mathrm{~B}$ ). Accordingly, myocyte CSA did not differ at PP9 $\left(195 \pm 100 \mu \mathrm{m}^{2} L M N A^{\mathrm{GT} /-}\right.$ vs. $259 \pm 59 \mu \mathrm{m}^{2}$ WT $\left.\mathrm{p}>0.05\right)$, but was significantly lower at PP15 $\left(270 \pm 55 \mu \mathrm{m}^{2} L M N A^{\mathrm{GT}-/-}\right.$ vs. $500 \pm 50$ $\mu \mathrm{m}^{2}$ WT $\mathrm{p}<0.01$; Figure $4 \mathrm{C}$ ). Consistent with these findings, $L M N A^{\mathrm{GT}-/}$ mice displayed reduced muscular strength (lag time dropping from grid: $0.9 \pm 0.5 \mathrm{sec} L M N A^{\mathrm{GT}-/}$ vs. $6.7 \pm 1.4 \mathrm{sec}$ WT PP15; $p<0.05$; Figure 4D). Histological analysis did not show any overt signs of fibrosis, as assessed by Sirius Red staining and Z-disc deorganization assessed by TEM (data not shown). These observations suggest that lamin $A / C$ is important for maintaining sketelal muscle mass and strength.

\section{Adipogenic Phenotype}

As pathological findings included reduced subcutaneous adipose tissue at PP15 in LMNA ${ }^{\mathrm{GT}-/-}$ mice, we further investigated the role of lamin $\mathrm{A} / \mathrm{C}$ 
during early post-natal differentiation and maturation of adipose tissue. Subcutaneous adipose deposits at PP5 were comparable in weight for $\mathrm{WT}$ and $L M N A^{\mathrm{GT}-/-}$ mice (weight corrected for tibia length: $0.38 \pm 0.35$ $\mathrm{mg} / \mathrm{mm} L M N A^{\mathrm{GT}-/-}$ vs. $0.40 \pm 0.17 \mathrm{mg} / \mathrm{mm}$ WT p>0.05; Figure $5 \mathrm{~A}$ ). During early post-natal development both $\mathrm{WT}$ and $L M N A^{\mathrm{GT+}+-}$ mice increase their subcutaneous fat storage (PP14 $1.13 \pm 0.17 \mathrm{mg} / \mathrm{mm}$ WT $\mathrm{p}<0.05$ compared to PP5), while this storage was severely impaired in $L M N A^{\text {GT-/- }}$ mice (PP14 $0.62 \pm 0.16 \mathrm{mg} / \mathrm{mm}, \mathrm{p}<0.05$ compared to age-matched WT). Gonadal fat patches and subscapular brown adipose tissue were present in all genotypes and macroscopically showed no obvious mass reduction.

A

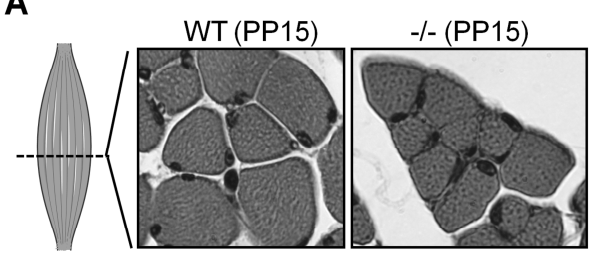

C

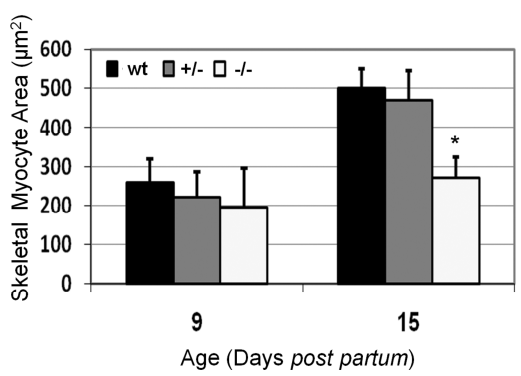

B

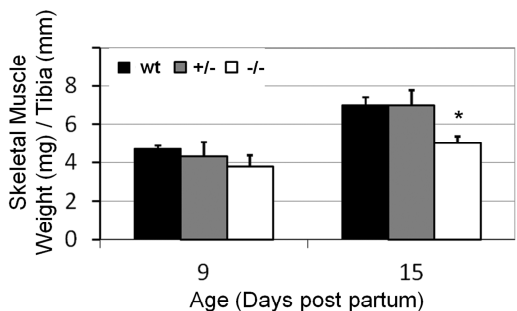

D

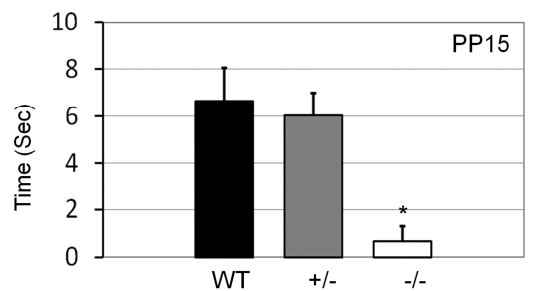

Figure 4. Post-natal skeletal muscle morphology and functioning in the $L M N A^{\mathrm{GT}-\mathrm{I}^{-}}$model. (A) Typical example of a haematoxylin and eosin (H\&E) staining on quadriceps skeletal muscle sections of $L M N A^{\mathrm{GT}-/}$ and WT sibling (PP15). (B) Total quadriceps muscle weight normalized to tibia length assessed at PP9 and PP15 in $L M N A^{\mathrm{GT-} /}, L M N A^{\mathrm{GT+} /-}$ and WT mice. (C) Quantification of the cross sectional area of quadriceps skeletal muscle myocytes for all 3 genotypes (PP9, PP15). (D) Assessment of skeletal muscle strength: time (in seconds) indicates the lag time until a mouse, hanging upside down, releases the grid; Asterisks in Figure 4 indicate significant differences for $L M N A^{\mathrm{GT} /-}$ vs. both $L M N A^{\mathrm{GT+/}}$ and WT values $(\mathrm{P}<0.05 ; \mathrm{n}=4)$. 
To directly investigate whether lack of subcutaneous adipose tissue could be caused by differentiation defects, E12.0 mouse embryonic fibroblasts (MEFs) were harvested from all 3 genotypes and adipogenic differentiation was induced ex vivo. Oil Red O lipid staining was substantially reduced upon complete loss of lamin A/C (0.284 \pm 0.033 $L M N A^{G T-/}$ vs. $0.615 \pm 0.159$ WT at 16 days post induction, $p<0.05$; Figure $5 B, C)$. These findings suggest that that loss of lamin $A / C$ results in impaired adipogenic differentiation and decreased post-natal subcutaneous fat deposition.

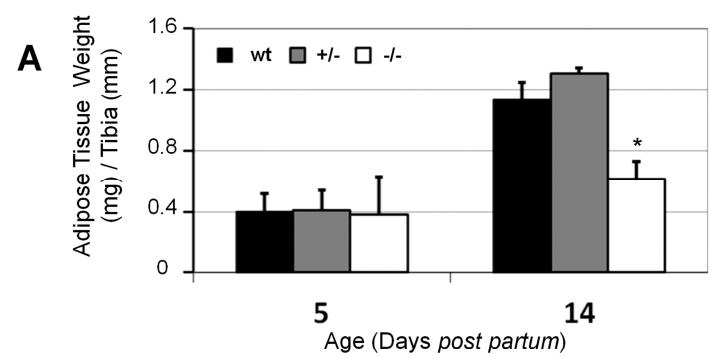

B

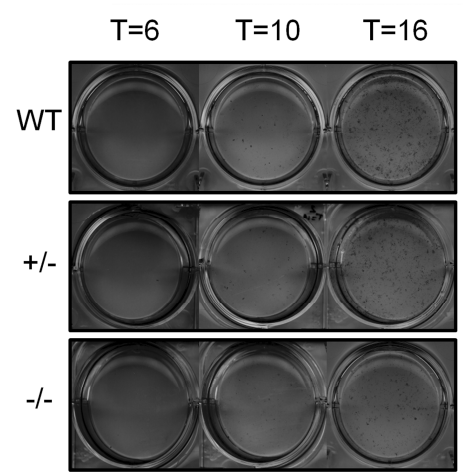

C

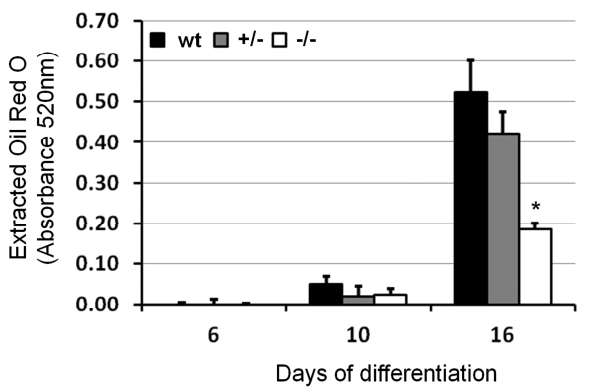

Figure 5. Adipogenic capacity of the LMNA ${ }^{\mathrm{GT}-{ }^{-}}$murine model. (A) Quantification of 5 and 14 day old subcutaneous adipose tissue deposits normalized to tibia length (See Material \& Methods). (B) Representative examples of Oil Red $O$ staining at 16 days of differentiation for all genotypes. (C) Quantification of Oil Red $O$ staining at 6,10, and 16 days post induction. Asterisks in Figure 5 indicate significant differences for $L M N A^{\mathrm{GT}-\mathrm{F}^{-}}$vs. both $\angle M N A^{\mathrm{GT+/-}}$ and $\mathrm{WT}$ values $(\mathrm{P}<0.05 ; \mathrm{n}=3)$. 


\section{Metabolic Parameters}

As loss of subcutaneous adipose tissue is expected to influence general metabolism, a metabolic fingerprint was taken at PP12 and PP16 by determining the status of carbohydrate, fat and protein metabolism. Glucose metabolism was determined by measuring blood levels of glucose (the major carbohydrate energy source for the glycolytic pathway), liver glycogen levels (energy storage) and serum lactate (a byproduct of anaerobic metabolism). Only lactate levels were reduced at

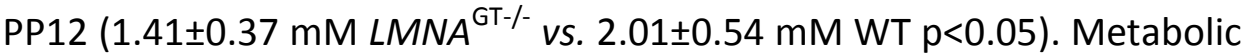
complications were observed at PP16 (Table 2) as $L M N A^{\mathrm{GT} /-}$ mice became hypoglycemic $\left(1.88 \pm 0.97 \mathrm{mM} L M N A^{\mathrm{GT}-/}\right.$ vs. $5.40 \pm 1.54$ WT $\mathrm{p}<0.05)$ while serum lactate levels remained normal. In further agreement with lowered blood glucose levels, glycogen deposits in the liver were undetectable in $L M N A^{\mathrm{GT}-/-}$ mice at PP18 in contrast to WT and $\angle M N A^{\mathrm{GT}+/}$ mice (Figure $\mathrm{S} 2$ ).

Fat/Triglycerides metabolism was monitored by determining the acylcarnitine profiles (carnitine-coupled free fatty acid (FFA)) substrates for ß-oxidation) (18), and by measuring serum levels of the ketone body betahydroxybutyrate (by-product of fatty acid catabolism; alternative energy source during hypoglycemia). At PP12 most of these parameters were normal, with the exception of lowered C12 carnitine levels $\left(0.08 \pm 0.01 \mathrm{pmol} / \mathrm{mg} L M N A^{\mathrm{GT}-/}\right.$ vs. $0.013 \pm 0.02 \mathrm{pmol} / \mathrm{mg}$ WT $\left.\mathrm{p}<0.05\right)$. Metabolic complications became apparent in $L M N A^{\mathrm{GT}-/}$ mice at PP16 (Table 2), as elevated levels of the ketone body betahydroxybutyrate $\left(1.94 \pm 0.18 \mathrm{mM} L M N A^{\mathrm{GT} /-}\right.$ vs. triglycerides into fatty acids and ketone bodies. An elevated ratio of $1.26 \pm 0.21 \mathrm{mM} W T \mathrm{p}<0.05)$ indicate increased conversion of free carnitine to bound carnitine $(0.72 \pm 0.17$ $L M N A^{\mathrm{GT}-/-}$ vs. $\left.0.038 \pm 0.05 \mathrm{WT} \mathrm{p}<0.01\right), \mathrm{C} 2$ acetyl-carnitine $(18.86 \pm 1.90$ $\mathrm{pmol} / \mathrm{mg} L M N A^{\mathrm{GT} /-}$ vs. $\left.14.67 \pm 1.73 \mathrm{pmol} / \mathrm{mg} \mathrm{WT} \mathrm{p}<0.05\right)$ and $\mathrm{C} 16$ palmitoyl-carnitine $\left(0.86 \pm 0.13 \mathrm{pmol} / \mathrm{mg} \quad L M N A^{\mathrm{GT} /-}\right.$ vs. $0.57 \pm 0.15$ $\mathrm{pmol} / \mathrm{mg}$ WT $\mathrm{p}<0.05$ ) indicate increased FFA transport across the mitochondrial membrane. 
Table 2. Post-natal metabolic fingerprint of the LMNAGT $^{-/-}$mouse.

\begin{tabular}{|c|c|c|c|c|c|}
\hline Age & Parameter Measured & $N=$ & $w t$ & $+/-$ & $-/-$ \\
\hline \multirow{4}{*}{ PP9 } & Body Core Temperature $\left({ }^{\circ} \mathrm{C}\right)$ & 5 & $31.5 \pm 2.0$ & $32.1 \pm 1.5$ & $29.5 \pm 0.9 *$ \\
\hline & Creatin Kinase (units/liter) & 4 & $646 \pm 169$ & & $551 \pm 231$ \\
\hline & Creatinine $(\mu \mathrm{M})$ & 4 & $8.0 \pm 1.7$ & & $7.1 \pm 1.2$ \\
\hline & Ureum (mM) & 4 & $8.7 \pm 2.1$ & & $9.2 \pm 1.2$ \\
\hline \multirow{11}{*}{ PP12 } & Glucose (mM) & 6 & $3.20 \pm 0.83$ & $3.23 \pm 0.80$ & $3.08 \pm 1.24$ \\
\hline & Lactate (mM) & 6 & $2.01 \pm 0.54$ & $2.52 \pm 1.14$ & $1.41 \pm 0.37^{*}$ \\
\hline & Betahydroxybutyrate (mM) & 6 & $0.97 \pm 0.22$ & $1.02 \pm 0.20$ & $0.90 \pm 0.25$ \\
\hline & C0, Free Carnitine (pmol/mg) & 6 & $31.88 \pm 8.96$ & $32.03 \pm 2.85$ & $29.07 \pm 1.70$ \\
\hline & $\mathrm{C} 2$, Non-Free Carnitine (pmol/mg) & 6 & $10.39 \pm 3.60$ & $11.52 \pm 2.69$ & $9.97 \pm 1.33$ \\
\hline & C0/C2, Free Carnitine/Non-free Carnitine & 6 & $0.32 \pm 0.06$ & $0.31 \pm 0.12$ & $0.34 \pm 0.05$ \\
\hline & C2, Acetyl-carnitine(pmol/mg) & 6 & $10.39 \pm 3.60$ & $11.52 \pm 2.69$ & $9.97 \pm 1.33$ \\
\hline & C5 carnitine (pmol/mg) & 6 & $0.38 \pm 0.22$ & $0.36 \pm 0.21$ & $0.38 \pm 0.06$ \\
\hline & C12 Carnitine (pmol/mg) & 6 & $0.13 \pm 0.02$ & $0.12 \pm 0.02$ & $0.08 \pm 0.01 *$ \\
\hline & C16, palmitoyl-carnitine (pmol/mg) & 6 & $0.75 \pm 0.42$ & $0.59 \pm 0.22$ & $0.40 \pm 0.09$ \\
\hline & C18:1, Oleoyl-carnitine (pmol/mg) & 6 & $0.26 \pm 0.19$ & $0.20 \pm 0.11$ & $0.12 \pm 0.05$ \\
\hline \multirow{4}{*}{ PP15 } & Body Core Temperature $\left({ }^{\circ} \mathrm{C}\right)$ & 5 & $32.5 \pm 1.0$ & $32.5 \pm 1.8$ & $27.5 \pm 0.8^{*}$ \\
\hline & Creatin Kinase (units/liter) & 4 & $667 \pm 310$ & & $1561 \pm 287^{*}$ \\
\hline & Creatinine $(\mu \mathrm{M})$ & 4 & $9.6 \pm 2.3$ & & $8.75 \pm 0.9$ \\
\hline & Ureum (mM) & 4 & $8.9 \pm 3.3$ & & $10.2 \pm 3.9$ \\
\hline \multirow{11}{*}{ PP16 } & Glucose (mM) & 4 & $5.40 \pm 1.54$ & $4.94 \pm 1.11$ & $1.88 \pm 0.97^{*}$ \\
\hline & Lactate (mM) & 4 & $3.28 \pm 0.65$ & $2.50 \pm 0.62$ & $2.81 \pm 0.44$ \\
\hline & Betahydroxybutyrate (mM) & 4 & $1.26 \pm 0.21$ & $1.26 \pm 0.17$ & $1.94 \pm 0.18^{*}$ \\
\hline & C0, Free Carnitine (pmol/mg) & 4 & $39.28 \pm 9.06$ & $33.01 \pm 4.01$ & $26.73 \pm 2.6^{*}$ \\
\hline & $\mathrm{C} 2$, Non-Free Carnitine (pmol/mg) & 4 & $14.76 \pm 1.73$ & $12.40 \pm 2.06$ & $18.86 \pm 1.90^{*}$ \\
\hline & C0/C2, Free Carnitine/Non-free Carnitine & 4 & $0.38 \pm 0.05$ & $0.38 \pm 0.04$ & $0.72 \pm 0.17^{*}$ \\
\hline & C2, Acetyl-carnitine(pmol/mg) & 4 & $14.67 \pm 1.73$ & $12.40 \pm 2.06$ & $18.86 \pm 1.90^{*}$ \\
\hline & C5 carnitine (pmol/mg) & 4 & $0.41 \pm 0.28$ & $0.25 \pm 0.13$ & $0.40 \pm 0.20$ \\
\hline & C12 Carnitine (pmol/mg) & 4 & $0.15 \pm 0.03$ & $0.14 \pm 0.05$ & $0.23 \pm 0.12$ \\
\hline & C16, palmitoyl-carnitine (pmol/mg) & 4 & $0.57 \pm 0.15$ & $0.57 \pm 0.08$ & $0.86 \pm 0.13^{*}$ \\
\hline & C18:1, Oleoyl-carnitine (pmol/mg) & 4 & $0.14 \pm 0.06$ & $0.17 \pm 0.06$ & $0.25 \pm 0.17$ \\
\hline
\end{tabular}

A metabolic profile was determined on blood samples by assessing glucose, lactose, betahydroxybutyrate and various acylcarnitines levels at day PP12 and PP16. Body core temperature, creatinin, urea and creatin kinase levels were assessed at day PP9 and PP15. Asterisks indicate significant differences for LMNA ${ }^{\mathrm{GT} /-}$ vs. both $\mathrm{LMNA}^{\mathrm{GT}+/-}$ and WT values $(P<0.05)$. 
As general markers for protein catabolism, creatin kinase, creatinin and ureum were determined. Blood creatin kinase levels (marker for muscle

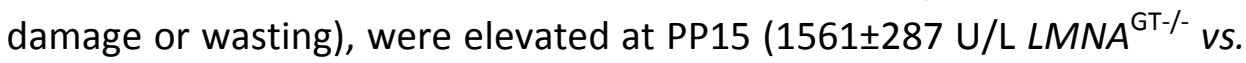
$667 \pm 310 \mathrm{U} / \mathrm{L} W T \mathrm{p}<0.05)$, but not at PP9. However, urea and creatinin levels (indicators of protein breakdown and kidney functioning), did not differ significantly (urea: $11.1 \pm 1.5 \mathrm{mmol} / \mathrm{L} L M N A^{\mathrm{GT}-/-}$ vs. $9.1 \pm 1.7 \mathrm{mmol} / \mathrm{L}$ WT; creatinin: $8.2 \pm 1.3 \mu \mathrm{mol} / \mathrm{L} L M N A^{\mathrm{GT} /-}$ vs. 9.7 $\pm 1.2 \mu \mathrm{mol} / \mathrm{L}$ WT; PP15; $\mathrm{p}>0.05)$. In addition to these metabolic parameters, core body temperature was lower both at PP9 $\left(29.5 \pm 0.9{ }^{\circ} \mathrm{C} L M N A^{\mathrm{GT}-/-}\right.$ vs. $31.5 \pm 2.0$ ${ }^{\circ} \mathrm{C}$ WT $\left.\mathrm{p}<0.05\right)$ and PP15 $\left(27.5 \pm 0.8{ }^{\circ} \mathrm{C} L M N A^{\mathrm{GT} /-}\right.$ vs. $32.5 \pm 1.0{ }^{\circ} \mathrm{C}$ WT $\mathrm{p}<0.01)$.

As the metabolic fingerprint and the expression array analysis (Figure 2B) supported an abnormal metabolic phenotype, morphology and function of mitochondria was assessed. Transmission electron microscopy revealed no differences in mitochondrial morphology (Figure S3A). Mitochondrial DNA copy numbers were comparable in both heart and quadriceps skeletal muscle at PP15 ( $p>0.05$; Figure S3B). Electron transport chain subcomplex activities were determined in LV tissue at PP15 and were shown to be almost fully comparable between WT and $\angle M N A^{\mathrm{GT} / \mathrm{-}}$ mice (Figure S3B). A slight elevation in the amount of mitochondria was observed, however (CS 1712 \pm 307 LMNA ${ }^{\mathrm{GT}-/-}$ vs. $1508 \pm 118$ WT $\mathrm{p}<0.05)$, and complex II activity was slightly, but significantly lowered $\left(0.114 \pm 0.00024 L M N A^{G T-/}\right.$ vs. $0.122 \pm 0.007$ WT $\mathrm{p}<0.01)$. These observations show the $L M N A^{G T-/}$ mice become severely catabolic in the $3^{\text {rd }}$ week post partum, without apparent abnormal mitochondrial function.

\section{Discussion}

Lamin A/C expression is developmentally regulated and has been specifically linked to early mesenchymal commitment ${ }^{4-6}$. Little is known, however, about the effect of A-type lamins in embryonic and early postnatal development. We have generated a novel $L M N A^{\mathrm{GT}-\mathrm{I}_{-}}$mouse model 
and demonstrate that loss of A-type lamins results in defective postnatal differentiation and defective maturation of adipose-, cardiac- and skeletal muscle tissue.

For many essential organs in mice, differentiation and maturation start during embryonic development and continues throughout early postnatal development ${ }^{21-23}$. Immunohistochemical studies revealed a diffuse lamin $A / C$ protein staining in connective tissue and myoblasts at E11.0 ${ }^{4-}$ 5. ${ }^{5}$. Lamin $A / C$ protein levels increase until day E14.0 and lamin A/C protein was only detected in a few more tissues during embryonic development from this age onwards. Lamin A/C was reported to be absent in epithelia of lung, liver, kidney, intestine, heart and brain until well after birth. Using a $\beta$-galactosidase reporter gene in our $L M N A^{\mathrm{GT}-/}$ mouse model we here report LMNA promoter activation, coinciding with the onset of tissue differentiation (E11.0) in somites, heart, liver and intestines and widespread LMNA expression by the end of in utero development (E18.0). In good agreement, promoter activity of the lamin A processing enzyme Zmpste24 in a GT mouse model partially mirrors the $L M N A$ promoter activity described here ${ }^{16}$. It is conceivable that previous immunohistochemical studies may have been limited in their sensitivity and specificity toward e.g. potential embryonic LMNA splicing variants. The detection of LMNA promoter activity from the earliest stages of tissue differentiation onward supports the importance of lamin $A / C$ in tissue differentiation and maturation. Although we cannot formally exclude discordant mRNA and protein synthesis, e.g. as a result of pre-/posttranslational regulation, our findings suggest that lamin A/C is expressed earlier than previously reported.

Despite apparently morphologically normal overall fetal development, the consequences of loss of lamin A/C becomes aparent during early post-natal development. By and large, post partum development in the absence of $L M N A$ reveals no gross phenotypical abnormalities from PP1 until PP6. The growth of $L M N A^{\mathrm{GT} /-}$ mice is retarded from PP7 to PP12 compared to $\mathrm{WT}$ and $L M N A^{\mathrm{GT}+\text { - }}$ siblings. We observe a prominent 
abnormal post-natal cardiac development in $L M N A^{\mathrm{GT}-/-}$ mice: whereas normal LV myocytes undergo post-natal hypertrophy upon cell cycle exit in the first week after birth ${ }^{21,24}$, cardiac hypertrophy is severely impaired in $L M N A^{\mathrm{GT}-/}$ mice. The comparable post partum decrease in cardiac proliferative activity (PCNA, Ki67) between WT and $L M N A^{\mathrm{GT}-/}$ siblings suggests altered hypertrophy is not caused by an inability of myocytes to exit the cell cycle. Instead, elevated expression of the hypertrophic markers $A N F$ and $B N P$, suggests that pro-hypertrophic signaling is increased in the absence of productive physiological cardiac hypertrophy. Finally, overall body growth ceases between PP13 and PP18 and BW decreases in $L M N A^{\mathrm{GT}-/-}$ mice. During this stage, skeletal muscle display hypotrophy and a prominent catabolic phenotype is manisfested, with reduced blood glucose (PP16), absent hepatic glycogen storage (PP18) and increased utilization of alternative energy sources (ketone bodies, FFA and muscle tissue; PP15-16). Although the direct cause of death is currently not clear, it most likely relates to a combination of muscle weakness and metabolic complications. Cardiac function appears not sufficiently impaired to explain premature death at this stage, but we cannot exclude the possibility that the short life-span of these mice prevents further development of cardiac malfunction. Decreased heart rate and core body temperature are typical side effects of a catabolic state. Both parameters decline in human anorexia nervosa patients as does blood pressure ${ }^{25}$. Progressive lowering of blood pressure and heart rate further deteriorates the condition during starvation ${ }^{26}$. This notion also provides an explanation for the detrimental effects of labetalol in $L M N A^{\mathrm{GT} / \mathrm{-}}$ mice, which furthermore demonstrates that in the end-stage increased sympathetic activity prolongs survival to some extent, possible by its effects on overall metabolism. Although it is unclear at this stage whether muscular wasting contributes to the abnormal metabolic state of $L M N A^{\mathrm{GT}-/}$ mice, the impaired adipogenic capacity upon loss of lamin $A / C$ provides a direct etiological connection to the defective energy metabolism. 
The $L M N A^{G T-/-}$ mouse complements previously established LMNA murine models. Using a conventional gene targeting strategy, Stewart and colleagues created a functional LMNA knock-out mouse (LMNA ${ }^{\mathrm{KO}-\sigma_{-}}$) which displays growth retardation starting 2-3 weeks post partum, followed by skeletal abnormalities, decreased subcutaneous adipose deposits, cardiac dilatation and, ultimately at 6-8 weeks post partum, life-threatening impaired cardiac function ${ }^{27-29}$. Considering the onset and progression of these symptoms over time, the overall phenotype of the $L M N A^{\mathrm{KO}-/}$ animals is comparatively milder than that of $L M N A^{\mathrm{GT}-/}$ mice. A possible explanation for the hypomorphic phenotype in $L M N A^{\mathrm{KO}-}$ 1- mice is the difference in targeting strategy between the models. In the contrast to the $L M N A^{\mathrm{GT}-/}$ mouse, where a GT sequence is introduced at the $L M N A^{\prime}$ 's second intron, in the $L M N A^{\mathrm{KO}-/}$ mouse the LMNA gene is disrupted in exon 8 . Northern blot analysis revealed expression of faster migrating $L M N A$ mRNAs, possibly indicative of truncated but potentially functional N-terminal $L M N A$ gene products in $L M N A^{\mathrm{KO}-/-}$ mice ${ }^{29}$. In the $\angle M N A^{\mathrm{GT}-/}$ mouse no partial $L M N A$ splicing products were detected the mRNA level besides the $L M N A-\beta$-geo fusion protein. This synthetic geneproduct does not induce obvious phenotypic defects as $L M N A^{G T+/-}$ mice were phenotypically indistinguishable from WT mice in all tested assays. Interestingly, the use of genetrap approaches was associated with more dramatic phenotypes compared to conventional gene targeting strategies including the lamin A processing enzyme Zmpste24 loss-of function mouse models ${ }^{16,30}$. Besides the targeting strategy, phenotypes may be affected by genetic background, as identical mutated LMNA gene products give rise to different laminopathies in humans ${ }^{31}$. In comparison

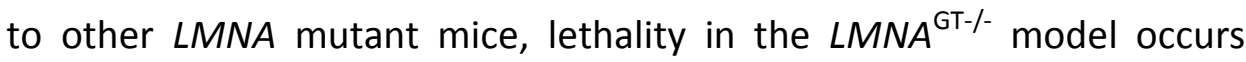
much earlier, and, notably, in the absence of cardiac functional defects and HGPS related bone abnormalities and hair loss ${ }^{13}$. Cardiac hypertropic defects may not have had sufficient time to progress to heart failure in our model, as other tissue defects dominate during postnatal development. 
The combined observations presented here show that the $L M N A^{\mathrm{GT}-/}$ mouse model is the most severe lamin A/C mouse model to date and, relevantly, our results show that loss of lamin $A / C$ induces severe and widespread defects in post-natal tissue maturation. This leads to an early post-natal and fully penetrant lethal phenotype, which is conceivably due to combined muscle weakness and metabolic complications, and occurs in the absence of a dilated cardiomyopathy or obvious progeroid phenotype. This novel LMNA mouse provides new opportunities to dissect the molecular and cellular mechanisms underlying the role of lamin $\mathrm{A} / \mathrm{C}$ in early post-natal development.

\section{Acknowledgements}

We thank S. Frints, MW. Schellings, C. van het Hoofd and A. Voets for technical expertise and help. We are indebted to JP. Cleutjens is thanked for help with quantification of myocyte cross-sectional areas, $\mathrm{H}$. Duimel for electron microscopy imaging, and P. Scaffidi and SG. Rane for adipogenic differentiation assays. Fast and reliable assistance in arranging animal experiments by members of the central animal facilities was instrumental for phenotypic analyses.This research was supported in part by the Intramural Research Program of the National Institutes of Health (NIH), NCl, Center for Cancer Research, grants from the Dutch Heart Foundation, NWO, ZonMW and the EU-KP7 grant 'Inheritance'. 


\section{References}

1. Prather RS, Sims MM, Maul GG, First NL, Schatten G. Nuclear lamin antigens are developmentally regulated during porcine and bovine embryogenesis. Biol Reprod 1989; 41:123-32.

2. Schatten G, Maul GG, Schatten H, Chaly N, Simerly C, Balczon R, et al. Nuclear lamins and peripheral nuclear antigens during fertilization and embryogenesis in mice and sea urchins. Proc Natl Acad Sci U S A 1985; 82:4727-31.

3. Stewart C, Burke B. Teratocarcinoma stem cells and early mouse embryos contain only a single major lamin polypeptide closely resembling lamin B. Cell 1987; 51:383-92.

4. Rober RA, Sauter H, Weber K, Osborn M. Cells of the cellular immune and hemopoietic system of the mouse lack lamins A/C: distinction versus other somatic cells. J Cell Sci 1990; 95 ( Pt 4):587-98.

5. Rober RA, Weber K, Osborn M. Differential timing of nuclear lamin A/C expression in the various organs of the mouse embryo and the young animal: a developmental study. Development 1989; 105:365-78.

6. Constantinescu D, Gray HL, Sammak PJ, Schatten GP, Csoka AB. Lamin A/C expression is a marker of mouse and human embryonic stem cell differentiation. Stem Cells 2006; 24:177-85.

7. Peter $M$, Nigg EA. Ectopic expression of an A-type lamin does not interfere with differentiation of lamin A-negative embryonal carcinoma cells. J Cell Sci 1991; 100 ( Pt 3):589-98.

8. Van Berlo JH, Voncken JW, Kubben N, Broers JL, Duisters R, van Leeuwen RE, et al. A-type lamins are essential for TGF-beta1 induced PP2A to dephosphorylate transcription factors. Hum Mol Genet 2005; 14:2839-49.

9. Wu Z, Wu L, Weng D, Xu D, Geng J, Zhao F. Reduced expression of lamin A/C correlates with poor histological differentiation and prognosis in primary gastric carcinoma. J Exp Clin Cancer Res 2009; 28:8.

10. Broers JL, Ramaekers FC, Bonne G, Yaou RB, Hutchison CJ. Nuclear lamins: laminopathies and their role in premature ageing. Physiol Rev 2006; 86:967-1008.

11. Lloyd DJ, Trembath RC, Shackleton S. A novel interaction between lamin A and SREBP1: implications for parial lipodystrophy and other laminopathies. Human Molecular Genetics 2002; 11:769-77.

12. Scaffidi P, Misteli T. Lamin A-dependent misregulation of adult stem cells associated with accelerated ageing. Nat Cell Biol 2008; 10:452-9.

13. Capell BC, Collins FS. Human laminopathies: nuclei gone genetically awry. Nat Rev Genet 2006; 7:940-52.

14. Junqueira LC, Bignolas G, Brentani RR. Picrosirius staining plus polarization microscopy, a specific method for collagen detection in tissue sections. Histochem J 1979; 11:447-55.

15. Schroen B, Leenders JJ, van Erk A, Bertrand AT, van Loon $M$, van Leeuwen RE, et al. Lysosomal integral membrane protein 2 is a novel component of the cardiac intercalated disc and vital for load-induced cardiac myocyte hypertrophy. J Exp Med 2007; 204:122735.

16. Pendas AM, Zhou Z, Cadinanos J, Freije JM, Wang J, Hultenby K, et al. Defective prelamin A processing and muscular and adipocyte alterations in Zmpste24 metalloproteinasedeficient mice. Nat Genet 2002; 31:94-9.

17. Soeters MR, Sauerwein HP, Faas L, Smeenge M, Duran M, Wanders RJ, et al. Effects of insulin on ketogenesis following fasting in lean and obese men. Obesity (Silver Spring) 2009; 17:1326-31. 
18. Houten SM. Metabolomics: Unraveling the chemical individuality of common human diseases. Ann Med 2009:1-6.

19. van den Bosch BJ, van den Burg CM, Schoonderwoerd K, Lindsey PJ, Scholte HR, de Coo $\mathrm{RF}$, et al. Regional absence of mitochondria causing energy depletion in the myocardium of muscle LIM protein knockout mice. Cardiovasc Res 2005; 65:411-8.

20. Szlachcic J, Hall WD, Tubau JF, Porter V, Vollmer C, Wollam G, et al. Left ventricular hypertrophy reversal with labetalol and propranolol: a prospective randomized, doubleblind study. Cardiovasc Drugs Ther 1990; 4:427-33.

21. Banerjee I, Fuseler JW, Price RL, Borg TK, Baudino TA. Determination of cell types and numbers during cardiac development in the neonatal and adult rat and mouse. Am J Physiol Heart Circ Physiol 2007; 293:H1883-91.

22. Hew KW, Keller KA. Postnatal anatomical and functional development of the heart: a species comparison. Birth Defects Res B Dev Reprod Toxicol 2003; 68:309-20.

23. Wu S, Platteau A, Chen S, McNamara G, Whitsett J, Bancalari E. Conditional Overexpression of Connective Tissue Growth Factor Disrupts Postnatal Lung Development. Am J Respir Cell Mol Biol 2009.

24. Soonpaa MH, Kim KK, Pajak L, Franklin M, Field LJ. Cardiomyocyte DNA synthesis and binucleation during murine development. Am J Physiol 1996; 271:H2183-9.

25. Gottdiener JS, Gross HA, Henry WL, Borer JS, Ebert MH. Effects of self-induced starvation on cardiac size and function in anorexia nervosa. Circulation 1978; 58:425-33.

26. Pirke KM. Central and peripheral noradrenalin regulation in eating disorders. Psychiatry Res 1996; 62:43-9.

27. Nikolova V, Leimena C, McMahon AC, Tan JC, Chandar S, Jogia D, et al. Defects in nuclear structure and function promote dilated cardiomyopathy in lamin A/C-deficient mice. The Journal of Clinical Investigation 2004; 113:357-69.

28. Cutler DA, Sullivan TS, Marcus-Samuels B, Stewart CL, Reitman ML. Characterization of Adiposity and Metabolism in Lmna-Deficient Mice. Biochemical and Biophysical Research Communications 2002; 291:522-27.

29. Sullivan T, Escalante-Alcalde D, Bhatt H, Anver M, Bhat N, Nagashima K, et al. Loss of Atype lamin expression compromises nuclear envelope integrity leading to muscular dystrophy. J Cell Biol 1999; 147:913-20.

30. Yang SH, Bergo MO, Toth $\mathrm{Jl}$, Qiao X, Hu Y, Sandoval S, et al. Blocking protein farnesyltransferase improves nuclear blebbing in mouse fibroblasts with a targeted Hutchinson-Gilford progeria syndrome mutation. Proc Natl Acad Sci U S A 2005; 102:10291-6.

31. Rankin J, Ellard S. The laminopathies: a clinical review. Clin Genet 2006; 70:261-74. 


\section{Supplemental Figures}

A

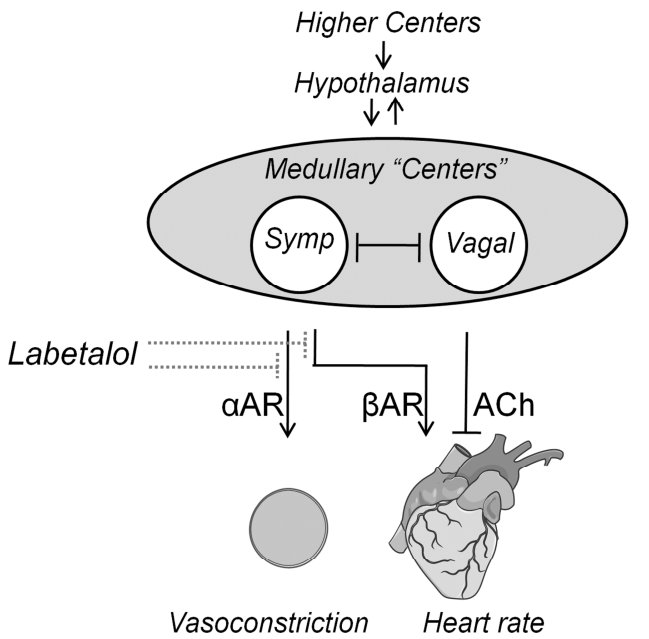

C

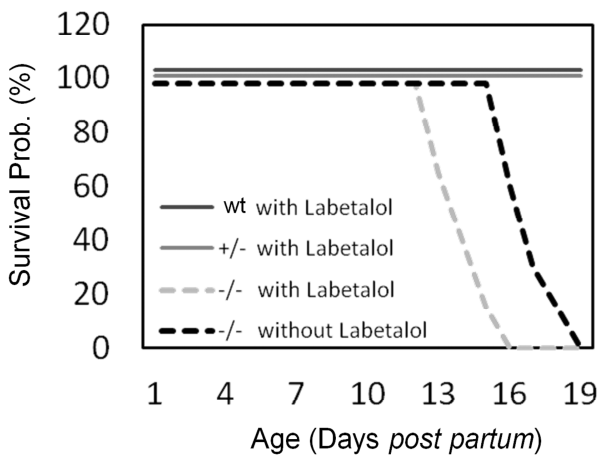

B

\begin{tabular}{|c|c|c|c|c|c|c|c|}
\hline \multirow[b]{2}{*}{ Age } & \multirow[b]{2}{*}{ Parameter Measured } & \multicolumn{2}{|c|}{ wt } & \multicolumn{2}{|c|}{$+/-$} & \multicolumn{2}{|c|}{$-/-$} \\
\hline & & $\begin{array}{c}\text { No } \\
\text { Labetalol }\end{array}$ & Labetalol & $\begin{array}{c}\text { No } \\
\text { Labetalol }\end{array}$ & Labetalol & $\begin{array}{c}\text { No } \\
\text { Labetalol }\end{array}$ & Labetalol \\
\hline \multirow{4}{*}{ PP13 } & P time $(\mathrm{ms})$ & $23.8 \pm 3.3$ & $25.3 \pm 1.8$ & $26.1 \pm 2.7$ & $25.2 \pm 2.6$ & $23.7 \pm 2.6$ & $29.8 \pm 3.2^{*}$ \\
\hline & QRS time (ms) & $14.0 \pm 2.4$ & $17.4 \pm 0.9^{*}$ & $13.7 \pm 0.8$ & $16.5 \pm 0.3^{*}$ & $14.4 \pm 1.7$ & $17.7 \pm 1.2^{*}$ \\
\hline & QT time (ms) & $37.2 \pm 9.6$ & $32.6 \pm 2.4$ & $33.7 \pm 3.1$ & $32.2 \pm 2.9$ & $33.2 \pm 5.9$ & $30.9 \pm 3.1$ \\
\hline & Heart Rate (bpm) & $345 \pm 30$ & $383 \pm 57$ & $422 \pm 58$ & $399 \pm 55$ & $396 \pm 51$ & $232 \pm 20^{*}$ \\
\hline
\end{tabular}

Figure S1. Cardiac effects of $\alpha$ - and $\beta$-adrenergic receptor antagonist treatment in $L M N A^{G T}$ mice. (A) Labetalol inhibits sympatic? activity by antagonizing $\alpha$ - and $\beta$-adrenergic receptors, thereby suppressing sympatic? stimulation of heart rate and vasoconstriction. Arrows and T-shaped ends indicate respectively stimulatory and inhibitory actions. (B) ECG analysis indicating heart rate ,P time, QRS duration and QT time 3 days after the start of subcutaneous labetalol or control injections in all genotypes (See Materials \& Methods). Asterisks in this figure indicate significant $(P<0.05 ; n=5)$ differences between labetalol and control injections within the same genotype. (C) Survival curve for $\angle M N A^{G T-/-}$ mice treated from PP10 onward with labetalol or control injections (dashed lines) versus survival curves for labetalol treated $L M N A^{\mathrm{GT}+{ }_{-}}$and WT siblings (straight lines). 

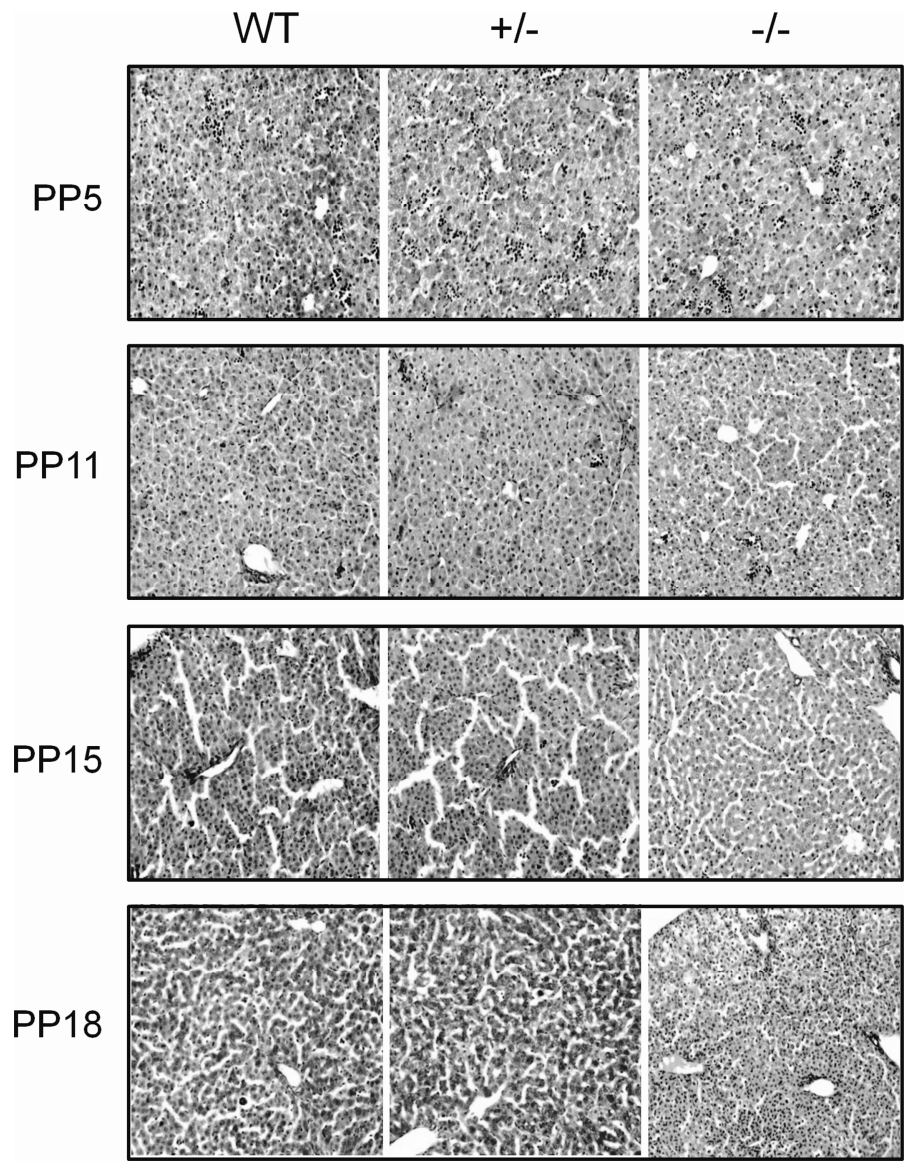

Figure S2. Post-natal hepatic glycogen storage in $L M N A^{G T-/}$ mice. Formalin fixed liver sections were Periodic Acid Shiff (PAS) stained to visualize glycogen deposits in a light purple/pink color. $L M N A^{\mathrm{GT}-/-}$ mice show absence of glycogen deposits at all ages investigated, whereasin WT and $L M N A^{\mathrm{GT}+\text {-- }}$ livers glycogen is detectable at day PP18. 
A
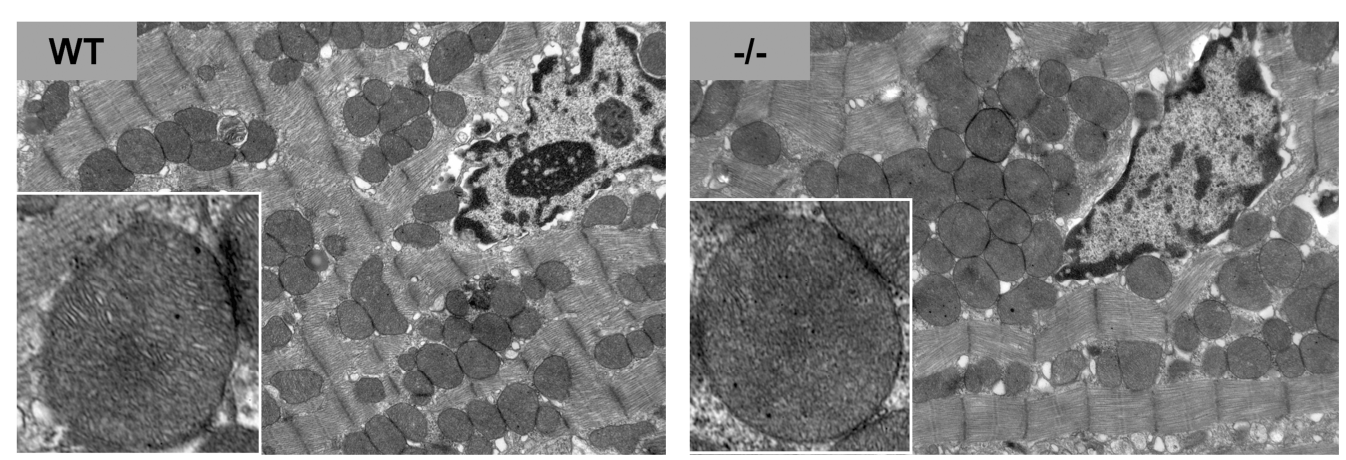

B

\begin{tabular}{|c|c|c|c|c|}
\hline Age & Parameter Measured & $\mathrm{N}=$ & wt & $\%$ \\
\hline \multirow{10}{*}{ PP15 } & Mitochondrial DNA copy number: & & & \\
\hline & Heart Left Ventricle & 4 & $6.93 \pm 2.18$ & $8.38 \pm 2.36$ \\
\hline & Skeletal Muscle & 4 & $4.08 \pm 1.24$ & $4.18 \pm 0.42$ \\
\hline & Mitochondrial Complex Activity: & & & \\
\hline & Citrate Synthase (CS) & 5 & $1508 \pm 118$ & $1712 \pm 307^{\star}$ \\
\hline & Complex I/CS & 5 & $0.182 \pm 0.013$ & $0.178 \pm 0.011$ \\
\hline & Complex II/CS & 5 & $0.122 \pm 0.007$ & $0.114 \pm 0.0024^{*}$ \\
\hline & Complex III/CS & 5 & $0.406 \pm 0.046$ & $0.345 \pm 0.122$ \\
\hline & Complex IVICS & 5 & $0.408 \pm 0.05$ & $0.372 \pm 0.06$ \\
\hline & Complex VICS & 5 & $0.461 \pm 0.028$ & $0.525 \pm 0.061$ \\
\hline
\end{tabular}

Figure S3. Mitochondrial morphology and functioning in $L M N A^{G T-/}$ mice. (A) Transmission Electron Microscopy pictures of left ventricle cardiac tissue at day PP15 WT and $L M N A^{\mathrm{GT}-{ }_{-}}$mice, showing cardiac muscle fiber and Z-disc orientation, and (in close-up) a typical example of a mitochondrion. (B) Mitochondrial DNA copy numbers in both quadriceps skeletal and cardiac muscle as determined at PP15 by RT-PCR. Asterisks indicate significant differences for $\mathrm{WT}$ and $L M N A^{\mathrm{GT}-/}$ values $(\mathrm{P}<0.05)$. 
178 | Chapter 6 


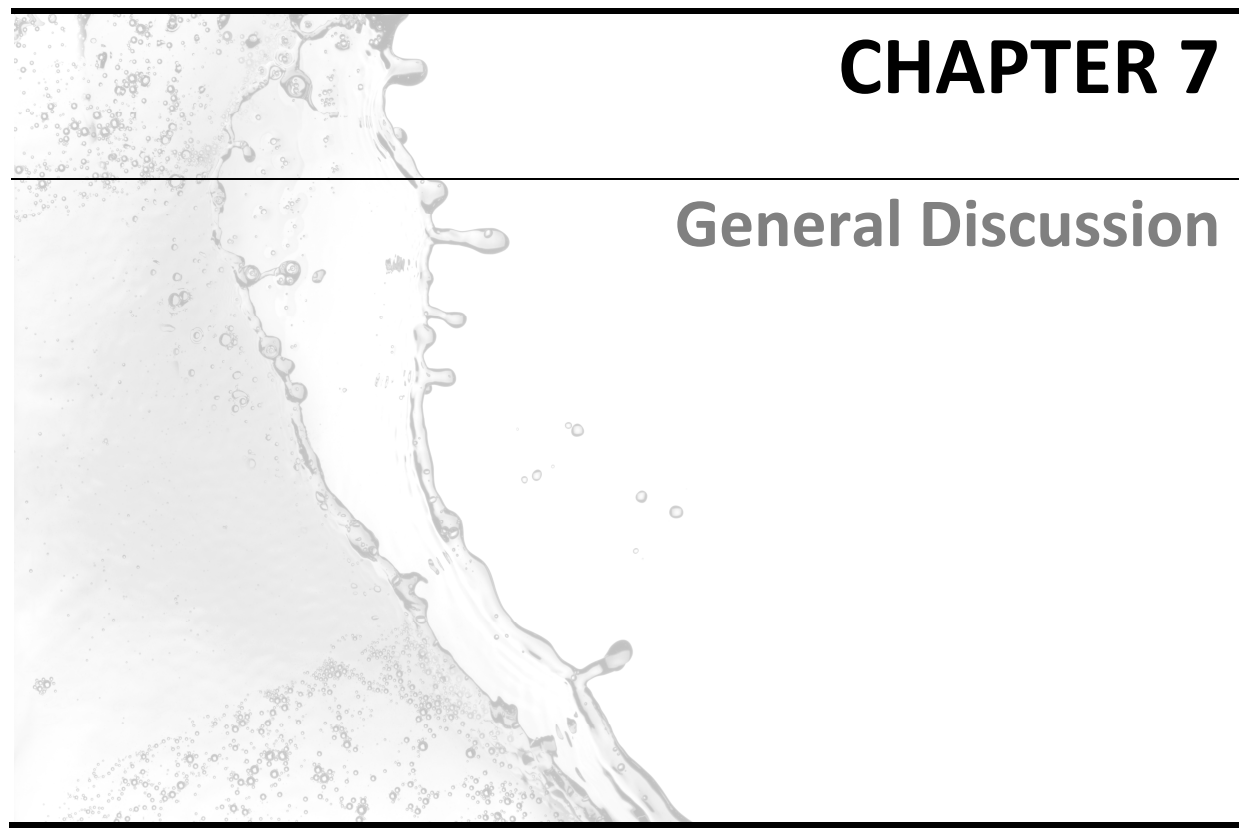


A-type lamins define the nuclear lamina and are key architectural proteins of the cell nucleus involved in various nuclear processes including transcription, DNA replication, DNA repair and cell signaling. ${ }^{1}$ Mutations in the LMNA gene, encoding for A-type lamins $A$ and $C$, are associated with 11 different diseases including muscular dystrophies, lipodystrophies, neuropathies and premature aging syndromes. ${ }^{2}$ The phenotypic diversity amongst these diseases, referred to as laminopathies, is hypothesized to be caused by mutation specific changes in lamin A protein- and chromatin interactions. Technological limitations hamper obtaining experimental evidence for a direct interaction of lamin A with chromatin in vivo, as well as mapping lamin A protein interactors in a proteome wide fashion. In this thesis novel techniques have been implemented to map lamin A-protein and -chromatin interactions in an unbiased approach, to identify differences in these interactomes for a progeria associated lamin A mutant and to study the biological relevance of $L M N A$ mutations in the context of chromatin organization and early postnatal differentiation.

\section{Lamin A protein- and chromatin-interactions}

Experimental approaches to identify lamin interactors are severely hampered by the biochemical properties of the nuclear lamina, classically defined as a non-ionic detergent, salt and nuclease insoluble peripheral nuclear structure ${ }^{3}$ (See Chapter 2). This insoluble character impedes immunoprecipitation (IP) studies, as solubilization by high concentrations of detergents disrupts protein- and chromatin interactions and hampers antigen-epitope binding. Alternative assays that do not depend on solubilization of intact complexes have been used instead, but suffer from specific drawbacks including low recovery, high false positives and nonphysiological conditions (Chapter 2). We therefore developed and applied a novel approach to identify lamin A-interacting proteins and chromatin. To map the full spectrum of both types of interactors we tagged lamin A with the biotin resembling OneSTrEP (OST) tag and expressed OST-tagged lamin A (OST-A) at endogenous levels in murine cells (Chapter 3 and 4). OST-A recapitulated endogenous lamin $A$ in terms of prelamin A processing, subnuclear localization, attachment of Emerin to the nuclear envelope (NE) and cell proliferative effects (Chapter 3 and 4). A mild cross-linking prior to 
OST precipitation enabled full solubilization and isolation of intact lamin A bound protein- and chromatin complexes. Although cross-linking is already commonly applied in chromatin immunoprecipitations (ChIP), it's combination with mass spectrometry (MS) is more recent ${ }^{4}$ and limited due to the enormous complexity of cross-linking reaction mixtures. ${ }^{5}$ To circumvent cross-linking induced artifacts we reverted cross-linking by extensive heating prior to analysis by mass spectrometry (MS; for protein interactions) or microarray technology (for DNA interactions). By OST pulldown we identified a total of 225 lamin A interacting proteins (Chapter 2) and 782 lamin A associated genes (Chapter 3).

We probed for the reliability of MS identified protein interactors by verifying several lamin A interactions with OST pulldown and western blot, including Ku70 and Trim28, two previously unknown interactors that were just above the cut-off of our stringent inclusion criteria. Both proteins interacted weakly but significantly with lamin A (Chapter 3 ). In addition, a comparative analysis with published data ${ }^{6-7}$ confirmed that our method reproducibly and reliably co-purified lamin A protein interactors. Half of the identified lamin A interactors were previously identified as NE components ${ }^{6-7}$ and their gene ontology (GO) signature highly resembles those of the NE proteome, including functions in NE structural organization, chromatin assembly and nuclear cytoplasmic transport via the nuclear pore complex (NPC) (Chapter 3). Observed GO differences with studies that biochemically purify and extract the NE, includes detection of interactions that are predicted to be short-lived (Zmpste24, proteasomes), as well as interactors involved in protein folding, processing and vesicle trafficking (Chapter 3). These differences are expected to occur due to preservation of temporal interactions by cross-linking and precipitation of the total lamin A pool by OST pulldown, including endoplasmic reticulum (ER) localized partially unfolded or unprocessed lamin A. In addition, ER localized proteins can as well diffuse to the continuous INM and anchor to the lamina. ${ }^{8}$ OST pulldown technology was furthermore used to confirm yeast two-hybrid ( $\mathrm{Y} 2 \mathrm{H})$ (Chapter 2) discovered novel interactions of the lamin A carboxy-terminus (amino acids 562-664) with Rbbp4, Rbbp7 and HDAC1, all members of the nucleosome remodeling and deacetylase (NURD) complex (Chapter 5). Rbbp4 is also a subunit of the CAF-1 complex, which assembles chromatin during DNA replication and DNA damage repair ${ }^{9}$, and together with $R b b p 7$ 
part of the Polycomb PRC2 complex. ${ }^{10}$ The NURD, CAF-1 and PRC2 complex are all involved in establishment of heterochromatin. ${ }^{9-12}$ Trim28 is associated with the Polycomb PRC1 complex (unpublished data) and involved in similar processes. ${ }^{13-14}$ Together with the observations that the nuclear periphery mostly contains heterochromatin and various LMNA mutations are associated with heterochromatin defects and DNA damage ${ }^{15-}$ ${ }^{16}$, these findings suggest that A-type lamins are crucial in maintaining higher-order chromatin organization.

In line with this, we identified in total 782 gene promoters that directly interact with lamin A by OST pulldown (Chapter 4). All examined lamin A targets preferentially localize at the nuclear periphery compared to nontargets, are genomically clustered and transcriptionally silenced (Chapter 4). Consistent with this, GO analysis revealed that lamin A-associated genes are typically involved in highly specified biological pathways, including the perception of smell and defensive responses to bacteria. Most likely proteins of both pathways, typically expressed by neurons and neutrophils respectively ${ }^{17-18}$, are not functional in fibroblast and cardiac myocytes used in our screen and hence expected to be transcriptionally silent. The presence of over 42 tissue and cell type specific transcription factor binding motifs (TFMs) in lamin A associated gene promoters further supports this notion (Chapter 4). Our findings are in keeping with DamID (Chapter 2) detected lamin B/gene interactions, which revealed sharply defined laminassociated domains (LADs) across the genome ${ }^{19}$, typically gene poor and low expressed regions, yet containing cell-type specific silenced gene clusters as well. ${ }^{20}$ To determine how crucial A-type lamin chromatin interaction is in peripheral positioning and regulation of gene expression, we monitored spatial positioning and gene expression upon RNAi-mediated knock-down of lamin A/C (Chapter 4). Upon lamin depletion target loci relocalize substantially to the nuclear interior, yet remain more frequently localized at the NE compared to non-targets. These findings were confirmed in a reversed approach, lamin A overexpression in LMNA knock-out (KO) MEFs, which showed increased peripheral localization for several lamin targets (Chapter 4). This supports a facilitating role for A-type lamins in recruitment of silenced genes to the nuclear periphery. Interactions with additional chromatin interacting nuclear lamina proteins, like emerin and Lamin B, contribute to this functional nuclear organization. ${ }^{19,}{ }^{21}$ Unchanged global 
expression levels for lamin A targets in a LMNA loss-of-function model, the lamin A gene trap (LMNA ${ }^{\mathrm{GT}-/}$ ) mouse (Chapter 6), argues that an escape from the repressive NE environment per se is not sufficient for gene activation. Most likely, loss of lamina-association is one of the multiple steps towards gene activation. Consistent with this, the CFTR locus' relocation due to activation and consequent internalization of neighboring genes by itself is insufficient for CFTR activation. ${ }^{22-23}$ For the olfactory receptor lamin A targets many additional steps necessary for stochastic activation have been described as well. ${ }^{18}$

\section{Progeria related changes in protein- and chromatin-interactions}

Hutchinson-Gilford Progeria Syndrome (HGPS) is a premature ageing disease hallmarked by global changes in chromatin, including loss of heterochromatin structure, altered patterns of histone modification, loss of key heterochromatic proteins and increased levels of persistent DNA damage. ${ }^{15-16,24-25}$ To elucidate the molecular mechanisms leading to Hutchinson-Gilford Progeria Syndrome (HGPS) we compared protein- and chromatin-interactions for lamin A and progerin, an HGPS causing lamin A mutant lacking 50 amino acids near the carboxy-terminus. OST tagged progerin recapitulated HGPS characteristic distortions of the nuclear lamina and global loss of LAP2 and HP1ץ nuclear levels ${ }^{16}$ (Chapter 3 and 4).

Of the identified 150 lamin A and 170 progerin interactors in MEFs, 35 proteins either had an increased affinity for lamin A (8 targets) or progerin (26 targets). These preferential interactors included proteins with GTPase activity and were involved in RNA metabolism or nucleocytoplasmic transport, including several NPC members (Nup98, Nup153, Tpr). Nup153 anchors NPCs to the nuclear lamina ${ }^{26-27}$ and directly interacts with $T p r$, which is involved in formation of NPC associated heterochromatin exclusion zones. ${ }^{28}$ A diminished interaction between progerin and Nup153 could explain a loss of NPC anchoring to the nuclear lamina ${ }^{26}$, subsequent NPC clustering and defective NPC mediated nuclear import, all associated with HGPS. ${ }^{27,} 29-30$ We also detected an increased interaction of progerin with paired related homeobox1 (Prx1), a transcriptional co-activator involved in skeletogenesis and vasculogenesis. ${ }^{31-32}$ Prx1 induces vascular smooth muscle cell proliferation and extracellular matrix (ECM) modeling by direct 
upregulation of tenascin C expression. ${ }^{31-32}$ Loss of Prx1 manifests itself in vertebrae and craniofacial skeletal defects, including absence of the mandible's zygomatic arches. ${ }^{32}$ These phenotypes highly resemble defects observed in progeria related mouse models, which suffer from osteogenesis imperfecta related fractures in vertebrae and mandible's zygomatic arch $^{33}$ as well as tenascin $C$ mediated progressive loss of vascular smooth muscle cells and ECM composition changes. ${ }^{34-35}$ Due to the mapping of protein interactions (Chapter 3 ) many more candidates potentially involved in HGPS aetiology are on the shelve and await confirmation and further investigation. An interesting candidate would be proliferating cellular nuclear antigen (PCna). Pcna regulates initiation of genomic DNA replication, which is hypothesized to be disturbed in HGPS by trapping of Pcna in intranuclear lamin aggregates, thereby contributing to premature ageing associated DNA damage. ${ }^{24,36}$ This hypothesis is in line with the increased interaction of Pcna with progerin observed by OST pulldown (Chapter 3).

In a second approach to identify a molecular basis for premature agingassociated chromatin defects, we probed for progerin interaction of the $\mathrm{Y} 2 \mathrm{H}$ identified and OST pulldown confirmed novel lamin A interactors Rbbp4 and $\mathrm{Rbbp7}$ (Chapter 5). For the $\mathrm{Y} 2 \mathrm{H}$ assay a carboxy terminal lamin A fragment (amino acids 562-664) was used that includes the 50 amino acids deleted in progerin (amino acids 607-657). Both NURD complex subunits interacted with lamin A but not with progerin, and showed reduced protein levels during normal and premature ageing (Chapter 5). This loss occurred in the same cells that showed HGPS characteristic global chromatin defects, including HP1 loss, loss of H3K9 tri-methylation ${ }^{15-16}$, increased transcription of pericentromeric satellite III repeats ${ }^{37}$ and increased levels of phosphorylated $\mathrm{H}_{2} \mathrm{AX} \mathrm{X}^{25}$, indicative of DNA double strand breaks (Chapter 5). A global loss of NURD components preceded the occurrence of persistent DNA damage. RNAi-mediated knock-down of both Rbbp4 and Rbbp7 was sufficient to recapitulate these HGPS chromatin defects. Other subunits of the NURD complex were also downregulated in old individuals and HGPS patients and a knock-down of several of these subunits caused HGPSassociated chromatin defects as well. These findings demonstrate how loss of several NURD components occurs during premature and normal ageing and contributes to structural chromatin defects ultimately leading up to the accumulation of DNA damage. The observed DNA damage might be a 
consequence of increased susceptibility of the affected chromatin to damage or, alternatively, might be caused by impaired DNA replication. ${ }^{38}$

To probe for other mechanisms through which progerin can affect chromatin organization we compared direct chromatin interactions for lamin A and progerin (Chapter 4). By OST-pulldown we identified 3 subclasses of chromatin interactors: those that interact with both lamin $A$ and progerin (common targets) and those interacting with only lamin A or progerin (differential targets). We identified several characteristics that distinguish these groups. Differentially interacting genes were least involved in cell-type specific biological processes and not enriched for any transcription factor binding motif, in contrast to common targets (Chapter 4). Furthermore, lamin $A /$ progerin commonly bound genes colocalized more frequently at the nuclear lamina, and this positioning was significantly less affected by addition of lamin A or progerin in comparison to differential interactors. Finally, genome-wide expression profiles show that, regardless of the presence of progerin, expression levels of common targets are lower than for genes that bind either lamin A or progerin. Unique interactors are still lower expressed compared to random genes though (Chapter 4). Overall this suggests that common targets are kept silent at the nuclear lamina, whereas differential interactors partially escape laminar interactions and are less likely to be influenced by repressive capacities. The observation that, in the absence of progerin, lamin A uniquely associated genes show significantly less expression then progerin uniquely interacting targets, which in this state do not interact with the lamina, further corroborates this notion. Introduction of progerin in LMNA ${ }^{\mathrm{KO}-/}$ MEFs increased the peripheral position of several progerin unique targets. Upon the introduction of progerin induced changes in chromatin organization and spatial positioning were insufficient to change expression levels of common or unique targets in wild-type MEFs. Most likely the absence of such effects is due to the presence of endogenous A-type lamins which partially counteract internalization of unique lamin $\mathrm{A}$ interactors and repositioning towards the nuclear rim for progerin unique targets. In line with this, spatial repositioning effects in wild-type MEFs were much less pronounced compared to $\mathrm{LMNA}^{\mathrm{KO}-/-} \mathrm{MEFs}$ (Chapter 4). A second factor contributing, as mentioned before, is the lack of additional factors needed for gene 
activation, which have been demonstrated to change gene interactions for B-type lamins ${ }^{21}$ and induce expression of cell-type specific gene clusters. ${ }^{20}$

\section{Biological relevance of A-type lamins in postnatal tissue differentiation}

The revealed interaction of the nuclear lamina with highly specialized and cell-type specific silenced genes (Chapter 4) and with cell-fate regulating chromatin remodeling proteins (NURD ${ }^{39}$, Trim28 ${ }^{40}$; Chapter 3 and 5) suggests a role for A-type lamins in cell differentiation. This notion is further corroborated by the finding that lamin $A / C$ expression is absent during embryonic development until the onset of tissue differentiation and maturation ${ }^{41-42}$ and in stem cells lamin $A$ is only expressed after differentiation induced loss of pluripotency ${ }^{43}$. Furthermore, several LMNA mutations interfere with adipogenic and osteogenic differentiation. ${ }^{44-45}$ In order to determine the biological relevance for A-type lamins in differentiation we developed and used a novel LMNA gene trap (LMNA ${ }^{\mathrm{GT}-\mathrm{I}^{-}}$) mouse model for LMNA loss-of-function (Chapter 6) in studying tissue differentiation defects during early postnatal development.

The gene trap enabled us to detect $L M N A$ promoter activity during embryonic development by $\beta$-galactosidase staining. LMNA was strongly stained for near the beginning of embryonic tissue differentiation (E11.0) in somites, heart, liver and intestines (Chapter 6). At the end of intra-uterine development (E18.0) LMNA promoter activity was macroscopically visible in all tissues. The detection of $L M N A$ promoter activity from the very early beginning of tissue differentiation onward supports the importance of lamin $\mathrm{A} / \mathrm{C}$ in tissue differentiation and maturation. Our findings are in contrast to previous studies, which are unable to detect lamin A/C protein in many tissues until well after birth by immunohistochemistry $(\mathrm{IHC}) .{ }^{41-42}$ Although we can't strictly exclude a discrepancy between mRNA production and protein expression (e.g. pre-/posttranslational regulation), the data presented here suggest that reported IHC studies may have been hampered by relative insensitivity of antisera used, or inability to detect potential embryonic $L M N A$ splicing variants. In support of our observations, promoter activity of the lamin A processing enzyme Zmpste24 in a GT mouse model resembles the $L M N A$ promoter described in Chapter $6 .{ }^{46}$ 
Table 1. LMNA mouse models

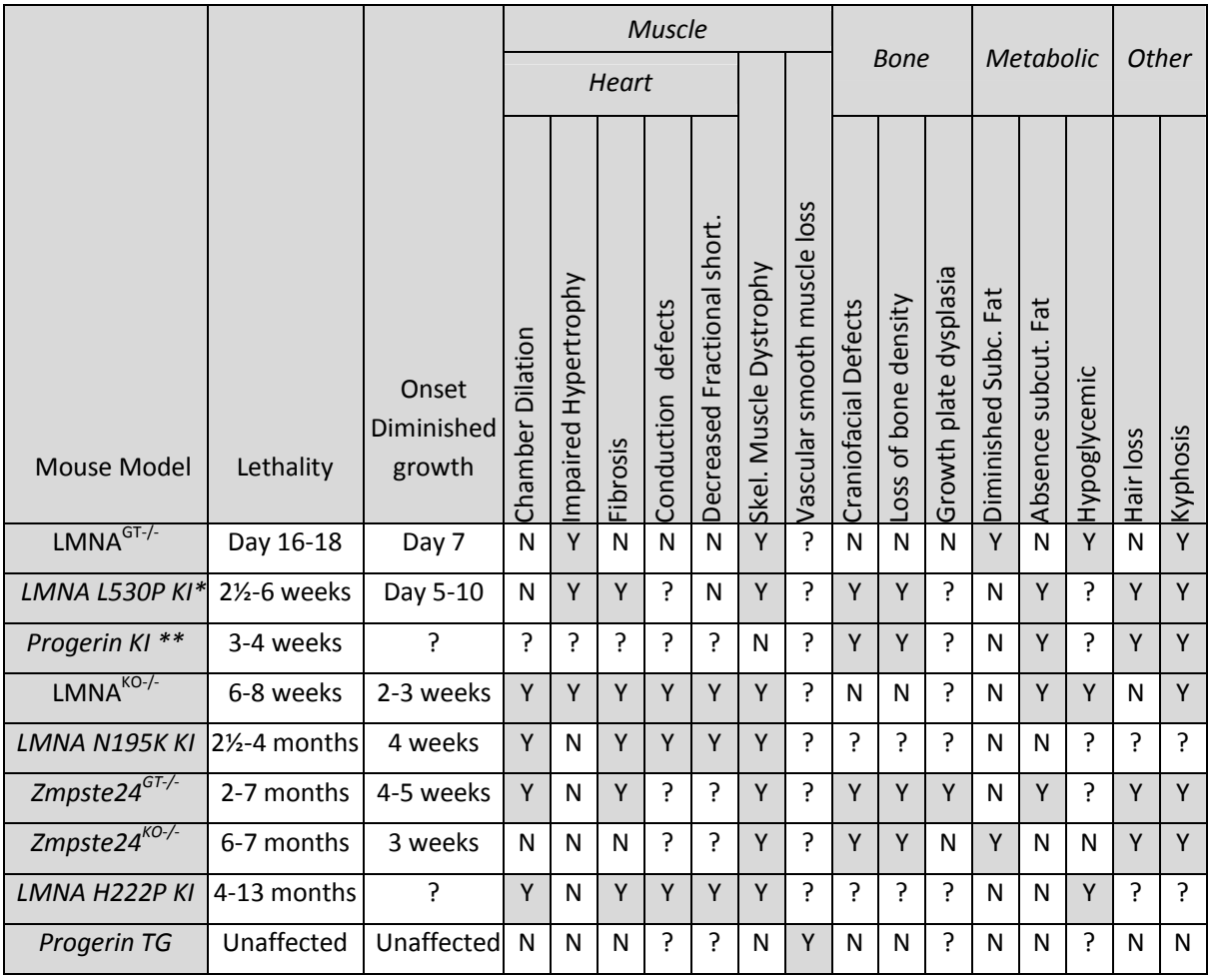

Legend: $\mathrm{Y}=$ Defect is present, $\mathrm{N}=$ Defect is absent, ?=It is not reported whether defect is present, $\mathrm{KO}=$ knock-out, $\mathrm{KI}=$ knock-in, TG=Transgene, GT=Gene trap; * LMNA transcripts lack either exon 9 or $9-12$ due to a splicing anomaly. Lamin $C$ absent, lamin A reduced levels; ${ }^{* *}$ Transgenic allele only produces progerin, no lamin A or C

Though no morphological defects were observed during embryonic development, the more pronounced the effect of loss of lamin $\mathrm{A} / \mathrm{C}$ became manifest during early postnatal development. $\mathrm{LMNA}^{\mathrm{GT} / \mathrm{-}}$ mice uniformly died within 3 weeks after birth with clear adipose, cardiac and skeletal muscle tissue defects (Chapter 6). Cardiac myocyte hypertrophy was impaired despite increased expression of hypertrophic markers. No cardiac functional defects were observed(Chapter 6). The increased expression of hypertrophy markers, which do not induce but mainly signify cardiac hypertrophy ${ }^{47}$, suggests that pro-hypertrophic signaling pathways may be increased in an attempt to induce the desired physiological hypertrophy. Skeletal muscles were hypotrophic and in addition LMNA ${ }^{\mathrm{GT}-/}$ mice displayed an impaired body growth, decreased subcutaneous adipose tissue and a catabolic blood profile several days before the occurrence of death. Metabolic defects can 
in part be explained due to a reduced adipogenic capacity, as adipogenic differentiation was found to be impaired in vitro (Chapter 6). This is in line with $L M N A$ mutations affecting adipogenic differentiation. ${ }^{44-45}$ It remains unclear whether observed muscle wasting contributes to the abnormal metabolic state of LMNA ${ }^{\text {GT-/ }}$ mice. Although an exact cause of death could not be pinpointed, death is likely due to a combination of muscle weakness and metabolic complications.

Observed tissue differentiation and maturation defects of the LMNA ${ }^{\text {GT- } /-}$ mouse complement previously established LMNA murine models (Table 1). Stewart and colleagues created a functional LMNA knock-out (LMNA ${ }^{\mathrm{KO}-\digamma_{-}}$) mouse ${ }^{5-7}$ which displays growth retardation starting 2-3 weeks post partum, followed in time by skeletal abnormalities, decreased subcutaneous adipose deposits, cardiac dilatation and ultimately at 6-8 weeks post partum lifethreatening impaired cardiac functioning ${ }^{5-7}$ (See Table 1 ). This phenotype is comparatively milder then for the LMNA ${ }^{\mathrm{GT}-/}$ mice considering onset and progression of symptoms over time (Chapter 6). A potential explanation for this discrepancy could be the place at which the LMNA gene was genetically modified. In LMNA ${ }^{G T-/}$ mouse a GT sequence is introduced after lamin $A^{\prime}$ 's first exon, while the LMNA gene is targeted at the $3^{\prime}$ end in the LMNA ${ }^{\mathrm{KO}-{ }^{\prime}-}$ mouse. The latter is more likely to permit expression of shorter but potentially functional partial $L M N A$ products from the $5^{\prime}$ end of the gene. Northern blot assays indeed showed faster migrating 10-fold lower expressed LMNA mRNA products in LMNA ${ }^{\mathrm{KO}-/}$ mice ${ }^{7}$. No shortened LMNA products were detected however at protein level ${ }^{7}$, possibly due to the absence of an $A B$ recognizable epitope. Interestingly, the use of GT sequences has been reported to cause more drastic phenotypes compared to conventional gene targeting strategies in Zmpste24 loss-of function mouse models as well ${ }^{3,9}$ (Table 1). Besides the chosen gene targeting strategy, phenotypes might be prone to differences in genetic background, as identical LMNA amino acid changes have been reported to give rise to different laminopathies in humans. ${ }^{14}$ The phenotype of the LMNA ${ }^{G T-/}$ mouse differs from existing dominant negative LMNA models, by a much earlier time at which lethality occurs and by the absence of cardiac functional defects and progeria related bone abnormalities and hair loss (Table 1). The absence of ageing related derangements can be explained by the fact that these are especially caused by dominant gain-of-function lamin A mutants, 
like progerin ${ }^{15}$ and the L530P mutation induced aberrant splicing products ${ }^{2}$, while the LMNA ${ }^{G T-/-}$ is a loss-of-function model system.

\section{Future research directions}

Many questions regarding the molecular mechanisms behind HGPS and other laminopathies still remain unanswered. For example, it is unknown which mechanisms are involved in the loss of proteins from the NURD complex and how altered chromatin modifications result in persistent DNA damage. The involvement of a specific pathway in loss of NURD complex members is suggested by the fact that progerin affects protein levels of several subunits of the NURD complex, but not of the other complexes, such as the CAF-1 or PRC2, that also contain Rbbp4 and Rbbp7. Increased DNA damage might simply be a consequence of increased susceptibility due to globally affected chromatin structure, or be caused by specific nuclear processes like DNA replication as elsewhere postulated. ${ }^{16}$ An experimental approach to identify common pathways involved in HGPS related loss of NURD components and DNA damage, is to identify and compare protein interactomes for several HGPS related mutations and define common derangements compared to wild-type lamin A. Such an approach assumes that defective lamin-protein interactions are the basis for HGPS aetiology. An even more unbiased way to define primary molecular defects in HGPS aetiology is to detect targets whose knock-down can rescue an HGPS phenotype. Developing a fluorescent HGPS-phenotype read-out enables pursuing this goal by high-throughput genome-wide RNAi screening combined with automized fluorescent microscopy .

In the context of protein- and chromatin-interactions an interesting question to address, is whether the presence of progerin also does affect the interactomes of wild-type lamin A or simply exerts dominant negative effects by interacting itself with different proteins and chromatin regions. In addition, it remains elusive to which regions outside of gene promoters Atype lamins interact. Upgrading OST pulldown studies by novel ChIP sequencing technology and comparing OST-lamin A interactomes in the absence and presence of untagged progerin can provide answers herein. The relevance of changes in chromatin interactions in the context of gene 
expression can further be studied in differentiation models that possess relevant sequential factors necessary for gene activation. A long term exciting project would be to compare protein- and chromatin-interactions between various tissues and disease stages by creating a OST-A knock-in mouse, especially relevant as laminopathies are highly tissue-specific and NE proteomes vary drastically amongst tissues. ${ }^{17}$

\section{Conclusions}

The research in this thesis has started to unravel the role of A-type lamins and the effect of LMNA mutations in the context of disruption of protein- or chromatin interactions, chromatin organization and early postnatal tissue differentiation. Combined, our findings demonstrate that A-type lamins are important for chromatin organization by directly interacting with chromatin, thereby facilitating a peripheral localization of highly cell-type specific silenced genes (Chapter 4). We expect these interactions to be part of a transcriptional memory system for differentiation specific gene programs, as has been described for B-type lamins ${ }^{18-19}$. Adipogenic, skeletal- an cardiac muscle tissue early postnatal differentiation and maturation defects upon loss of A-type lamins are in line with this (Chapter 6). An HGPS causing LMNA mutation specifically modifies chromatin- and protein-interactions (Chapter 3 and 5) and thereby contributes to aging-related disruption of NPC organization (Chapter 3), heterochromatin defects and DNA damage (Chapter 5). Our findings herein provide a relevant basis for ongoing research determining defective pathways that precede and are caused by observed chromatin defects. Furthermore, the molecular toolbox developed within this thesis can be used to systematically map chromatin- and protein interactions for various $L M N A$ mutations in order to identify novel disease mechanisms and, in time, develop possible cures for various laminopathies. 


\section{References}

1. Broers, J.L., et al., Nuclear lamins: laminopathies and their role in premature ageing. Physiol Rev, 2006. 86(3): p. 967-1008.

2. Capell, B.C. and F.S. Collins, Human laminopathies: nuclei gone genetically awry. Nat Rev Genet, 2006. 7(12): p. 940-52.

3. Foisner, R., Dynamic Connections of Nuclear Envelope Proteins to Chromatin and the Nuclear Matrix, in Nuclear Envelope Dynamics in Embryos and Somatic Cells, P. Collas, Editor. 2003.

4. Tang, X. and J.E. Bruce, A new cross-linking strategy: protein interaction reporter (PIR) technology for protein-protein interaction studies. Mol Biosyst, 2010. 6(6): p. 939-47.

5. Sinz, A., Chemical cross-linking and mass spectrometry to map three-dimensional protein structures and protein-protein interactions. Mass Spectrom Rev, 2006. 25(4): p. 663-82.

6. Schirmer, E.C., et al., Nuclear membrane proteins with potential disease links found by subtractive proteomics. Science, 2003. 301(5638): p. 1380-2.

7. Dreger, M., et al., Nuclear envelope proteomics: novel integral membrane proteins of the inner nuclear membrane. Proc Natl Acad Sci U S A, 2001. 98(21): p. 11943-8.

8. Ostlund, C., et al., Dependence of diffusional mobility of integral inner nuclear membrane proteins on A-type lamins. Biochemistry, 2006. 45(5): p. 1374-82.

9. Verreault, A., et al., Nucleosome assembly by a complex of CAF-1 and acetylated histones H3/H4. Cell, 1996. 87(1): p. 95-104.

10. Kuzmichev, A., et al., Histone methyltransferase activity associated with a human multiprotein complex containing the Enhancer of Zeste protein. Genes Dev, 2002. 16(22): p. 2893-905.

11. Zhang, Y., et al., Analysis of the NuRD subunits reveals a histone deacetylase core complex and a connection with DNA methylation. Genes Dev, 1999. 13(15): p. 192435.

12. Verreault, A., et al., Nucleosomal DNA regulates the core-histone-binding subunit of the human Hat1 acetyltransferase. Curr Biol, 1998. 8(2): p. 96-108.

13. Groner, A.C., et al., KRAB-zinc finger proteins and KAP1 can mediate long-range transcriptional repression through heterochromatin spreading. PLoS Genet, 2010. 6(3): p. e1000869.

14. Noon, A.T., et al., 53BP1-dependent robust localized KAP-1 phosphorylation is essential for heterochromatic DNA double-strand break repair. Nat Cell Biol, 2010. 12(2): p. 177-84.

15. Scaffidi, P. and T. Misteli, Reversal of the cellular phenotype in the premature aging disease Hutchinson-Gilford progeria syndrome. Nat Med, 2005. 11(4): p. 440-5.

16. Scaffidi, P. and T. Misteli, Lamin A-dependent nuclear defects in human aging. Science, 2006. 312(5776): p. 1059-63.

17. Lehmann, J., et al., Expression of human beta-defensins 1 and 2 in kidneys with chronic bacterial infection. BMC Infect Dis, 2002. 2: p. 20.

18. Lomvardas, S., et al., Interchromosomal interactions and olfactory receptor choice. Cell, 2006. 126(2): p. 403-13.

19. de Wit, E. and B. van Steensel, Chromatin domains in higher eukaryotes: insights from genome-wide mapping studies. Chromosoma, 2009. 118(1): p. 25-36.

20. Shevelyov, Y.Y., et al., The B-type lamin is required for somatic repression of testisspecific gene clusters. Proc Natl Acad Sci U S A, 2009. 106(9): p. 3282-7. 
21. Pickersgill, H., et al., Characterization of the Drosophila melanogaster genome at the nuclear lamina. Nat Genet, 2006. 38(9): p. 1005-14.

22. Zink, D., et al., Transcription-dependent spatial arrangements of CFTR and adjacent genes in human cell nuclei. J Cell Biol, 2004. 166(6): p. 815-25.

23. Sadoni, N., et al., Transcription-dependent spatial arrangements of CFTR and conserved adjacent loci are not conserved in human and murine nuclei. Chromosoma, 2008. 117(4): p. 381-97.

24. Goldman, R.D., et al., Accumulation of mutant lamin A causes progressive changes in nuclear architecture in Hutchinson-Gilford progeria syndrome. Proc Natl Acad Sci U S A, 2004. 101(24): p. 8963-8.

25. Liu, B., et al., Genomic instability in laminopathy-based premature aging. Nat Med, 2005. 11(7): p. 780-5.

26. Smythe, C., H.E. Jenkins, and C.J. Hutchison, Incorporation of the nuclear pore basket protein nup153 into nuclear pore structures is dependent upon lamina assembly: evidence from cell-free extracts of Xenopus eggs. EMBO J, 2000. 19(15): p. 3918-31.

27. Walther, T.C., et al., The nucleoporin Nup153 is required for nuclear pore basket formation, nuclear pore complex anchoring and import of a subset of nuclear proteins. EMBO J, 2001. 20(20): p. 5703-14.

28. Krull, S., et al., Protein Tpr is required for establishing nuclear pore-associated zones of heterochromatin exclusion. EMBO J, 2010.

29. Busch, A., et al., Nuclear protein import is reduced in cells expressing nuclear envelopathy-causing lamin A mutants. Exp Cell Res, 2009. 315(14): p. 2373-85.

30. Hubner, S., et al., Laminopathy-inducing lamin A mutants can induce redistribution of lamin binding proteins into nuclear aggregates. Exp Cell Res, 2006. 312(2): p. 171-83.

31. Jones, F.S., et al., Prx1 controls vascular smooth muscle cell proliferation and tenascin-C expression and is upregulated with Prx2 in pulmonary vascular disease. Circ Res, 2001. 89(2): p. 131-8.

32. ten Berge, D., et al., Prx1 and Prx2 in skeletogenesis: roles in the craniofacial region, inner ear and limbs. Development, 1998. 125(19): p. 3831-42.

33. Bergo, M.O., et al., Zmpste24 deficiency in mice causes spontaneous bone fractures, muscle weakness, and a prelamin A processing defect. Proc Natl Acad Sci U S A, 2002. 99(20): p. 13049-54.

34. Csoka, A.B., et al., Genome-scale expression profiling of Hutchinson-Gilford progeria syndrome reveals widespread transcriptional misregulation leading to mesodermal/mesenchymal defects and accelerated atherosclerosis. Aging Cell, 2004. $3(4):$ p. 235-43.

35. Varga, R., et al., Progressive vascular smooth muscle cell defects in a mouse model of Hutchinson-Gilford progeria syndrome. Proc Natl Acad Sci U S A, 2006. 103(9): p. 3250-5.

36. Musich, P.R. and Z. Zou, Genomic instability and DNA damage responses in progeria arising from defective maturation of prelamin A. Aging (Albany NY), 2009. 1(1): p. 2837.

37. Shumaker, D.K., et al., From the Cover: Mutant nuclear lamin A leads to progressive alterations of epigenetic control in premature aging. Proc Natl Acad Sci U S A, 2006. 103(23): p. 8703-8.

38. Peng, J.C. and G.H. Karpen, Epigenetic regulation of heterochromatic DNA stability. Curr Opin Genet Dev, 2008. 18(2): p. 204-11.

39. Fujita, N., et al., MTA3 and the Mi-2/NuRD complex regulate cell fate during $B$ lymphocyte differentiation. Cell, 2004. 119(1): p. 75-86. 
40. Rooney, J.W. and K.L. Calame, TIF1beta functions as a coactivator for C/EBPbeta and is required for induced differentiation in the myelomonocytic cell line U937. Genes Dev, 2001. 15(22): p. 3023-38.

41. Rober, R.A., et al., Cells of the cellular immune and hemopoietic system of the mouse lack lamins A/C: distinction versus other somatic cells. J Cell Sci, 1990. 95 ( Pt 4): p. 587-98.

42. Rober, R.A., K. Weber, and M. Osborn, Differential timing of nuclear lamin $A / C$ expression in the various organs of the mouse embryo and the young animal: a developmental study. Development, 1989. 105(2): p. 365-78.

43. Constantinescu, D., et al., Lamin A/C expression is a marker of mouse and human embryonic stem cell differentiation. Stem Cells, 2006. 24(1): p. 177-85.

44. Lloyd, D.J., R.C. Trembath, and S. Shackleton, A novel interaction between lamin A and SREBP1: implications for parial lipodystrophy and other laminopathies. Human Molecular Genetics, 2002. 11: p. 769-777.

45. Scaffidi, P. and T. Misteli, Lamin A-dependent misregulation of adult stem cells associated with accelerated ageing. Nat Cell Biol, 2008. 10(4): p. 452-9.

46. Pendas, A.M., et al., Defective prelamin A processing and muscular and adipocyte alterations in Zmpste24 metalloproteinase-deficient mice. Nat Genet, 2002. 31(1): p. 94-9.

47. Doust, J.A., et al., A systematic review of the diagnostic accuracy of natriuretic peptides for heart failure. Arch Intern Med, 2004. 164(18): p. 1978-84.

48. Kubben, N., et al., In preparation: Post-natal myogenic and adipogenic development defects and metabolic impairment upon loss of A-type lamins. 2010.

49. Mounkes, L.C., et al., A progeroid syndrome in mice is caused by defects in A-type lamins. Nature, 2003. 423: p. 298-301.

50. Yang, S.H., et al., Blocking protein farnesyltransferase improves nuclear blebbing in mouse fibroblasts with a targeted Hutchinson-Gilford progeria syndrome mutation. Proc Natl Acad Sci U S A, 2005. 102(29): p. 10291-6.

51. Yang, S.H., et al., A farnesyltransferase inhibitor improves disease phenotypes in mice with a Hutchinson-Gilford progeria syndrome mutation. J Clin Invest, 2006. 116(8): p. 2115-21.

52. Nikolova, V., et al., Defects in nuclear structure and function promote dilated cardiomyopathy in lamin A/C-deficient mice. The Journal of Clinical Investigation, 2004. 113: p. 357-369.

53. Cutler, D.A., et al., Characterization of Adiposity and Metabolism in Lmna-Deficient Mice. Biochemical and Biophysical Research Communications, 2002. 291: p. 522-527.

54. Sullivan, T., et al., Loss of A-type lamin expression compromises nuclear envelope integrity leading to muscular dystrophy. J Cell Biol, 1999. 147(5): p. 913-20.

55. Mounkes, L.C., et al., Expression of an LMNA-N195K variant of A-type lamins results in cardiac conduction defects and death in mice. Hum Mol Genet, 2005. 14(15): p. 216780.

56. Varela, I., et al., Accelerated ageing in mice deficient in Zmpste24 protease is linked to p53 signalling activation. Nature, 2005. 437(7058): p. 564-8.

57. Arimura, T., et al., Mouse model carrying H222P-Lmna mutation develops muscular dystrophy and dilated cardiomyopathy similar to human striated muscle laminopathies. Hum Mol Genet, 2005. 14(1): p. 155-69.

58. Rankin, J. and S. Ellard, The laminopathies: a clinical review. Clin Genet, 2006. 70(4): p. 261-74.

59. Scaffidi, P. and T. Misteli, Good news in the nuclear envelope: loss of lamin A might be a gain. J Clin Invest, 2006. 116(3): p. 632-4. 
194 | Chapter 7

60. Schirmer, E.C. and L. Gerace, The nuclear membrane proteome: extending the envelope. Trends Biochem Sci, 2005. 


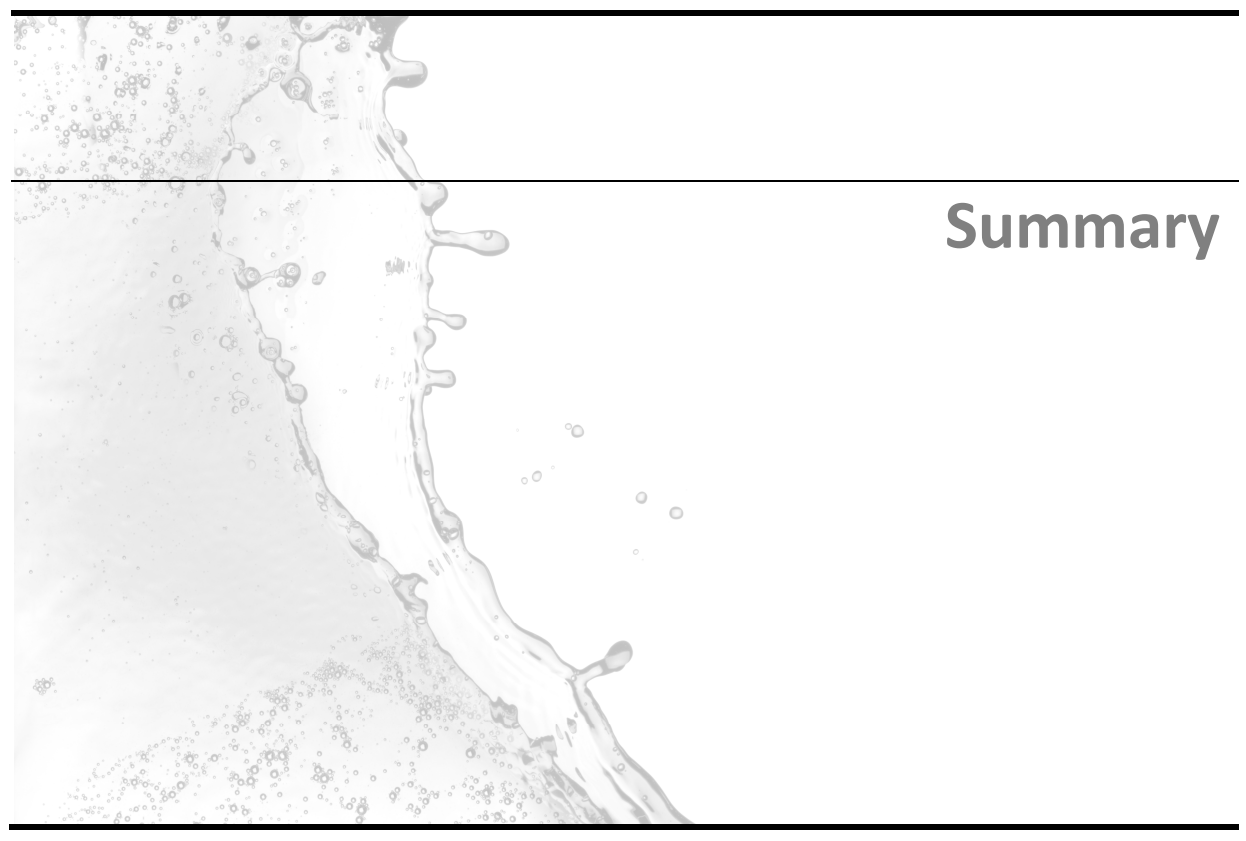


The nuclear periphery is marked by the nuclear envelope (NE), which is composed of an outer and inner nuclear membrane (ONM, INM, respectively) interrupted by nuclear pore complexes (NPC). The nuclear lamina lines the $N E$ and consists amongst others of the intermediate filament A-type (Lamin A, C) and B-type lamins (Lamin B1, B2), INM proteins anchoring the lamina to the NE and proteins interacting with chromatin and modifying its structure. Mutations in A type lamins, encoded by the LMNA gene, cause several human diseases collectively referred to as laminopathies, which include several types of muscular dystrophies, lipodystrophies, cardiomyopathies, neurological disorders and premature aging syndromes. At the basis for this thesis lies the major enigma how mutations in one gene can cause so much phenotypic diversity (Chapter 1). The working hypothesis of this thesis is that LMNA mutations affect specific lamina protein- and chromatin-interactions, thereby giving rise to laminopathy specific defects.

Chapter 2 describes the most commonly used tools to study lamina proteinand chromatin interactions and discusses the advantages and disadvantages inherent to each method. At the beginning of this thesis' research, in vivo interactions of A-type lamins with chromatin were hypothesized yet remained to be experimentally proven, and methodologies to identify protein interactions were severely hampered by the salt- and detergentinsoluble properties of the nuclear lamina. These biochemical properties forced researchers to make a trade-off between the degree of solubilization of lamina proteins and the amount of interactions potentially disrupted by used lysis conditions. Alternative assays circumventing this technical problem each have their specific drawbacks. In order to overcome limitations of available methods we developed a novel approach, the OneSTrEP (OST) pulldown, to study protein- and chromatin-interactions in a unbiased proteome- and genome-wide fashion. This technique consists of expressing lamin A tagged by a biotin resembling OST epitope, high affinity precipitation of fully solubilized but intact OST-lamin A complexes, and subsequent analysis of the isolated complexes for protein by mass spectrometry (MS) and for DNA by array technology.

We used this approach to identify proteins that interact with lamin A and its mutant isoform progerin, which causes the premature aging disorder 
Hutchinson-Gilford Progeria Syndrome (HGPS) (Chapter 3). We identified 150 lamin $A$ and 170 progerin-interacting proteins, including novel lamina interactors. Lamin A interactors are preferentially involved in NE structural organization, chromatin assembly and nuclear cytoplasmic transport via the NPC. Of the lamin A and progerin interactors 35 proteins preferentially bound to either lamin A or progerin. These differential interactors included several transcription factors, proteins with GTPase activity, and proteins involved RNA metabolism or nucleocytoplasmic transport, including several NPC members. The global identification of novel lamin A and progerin interactors has generated several leads for studies of specific interaction partners of lamin A and its mutants with the goal of uncovering the molecular mechanisms of tissue-specific laminopathies.

Next, we mapped genome-wide chromatin interactions of A-type lamins and identified 595 lamin A- and 552 progerin-associated gene promoters (Chapter 4). We find that lamin A preferentially binds peripherally localized, silent genes. This association facilitates, but does not determine, peripheral localization and loss of the interaction is not sufficient for gene activation. Further analysis identified multiple classes of common or uniquely lamin Aor progerin-associated genes. Basal expression of the unique categories correlates to their relative subnuclear location. Changes in subnuclear position upon introduction of progerin are insufficient to change basal expression levels. These observations demonstrate that lamin $A$ and progerin differently directly affect chromatin-lamina organization and that loss of peripheral localization and lamin association of endogenous gene loci is insufficient for their activation.

In order to identify a molecular basis for premature ageing characteristic defects in chromatin structure and accumulation of persistent DNA damage, we performed a yeast two-hybrid screen to identify differential lamin $\mathrm{A} /$ progerin interactors (Chapter 5 ). The nucleosome remodeling and deacetylase (NURD) complex subunits Rbbp4 and Rbbp7 were found to interact with lamin $A$, but not with progerin, and showed reduced protein levels during normal and premature ageing. Furthermore, the activity of histone deacetylase 1 (HDAC1), another component of the NURD complex, was reduced with aging. Silencing of individual NURD subunits recapitulated aging associated chromatin defects, which preceded the occurrence of 
persistent DNA damage. These findings demonstrate that loss of several NURD components occurs during premature and normal ageing and contributes to structural chromatin defects ultimately leading up to the accumulation of DNA damage.

Next, we probed for the biological relevance of A-type lamins in early postnatal tissue differentiation and maturation, relevant as LMNA expression is reversely correlated to the degree of differentiation and several LMNA mutations induce differentiation defects. To this end, we created a novel LMNA gene trap (LMNA ${ }^{\mathrm{GT}-\mathrm{-}}$ ) mouse model which combines loss of LMNA function with LMNA-controlled reporter gene expression (Chapter 6). We demonstrate early activity of the LMNA promoter during the beginning of embryonic tissue differentiation in heart, liver and somites. The loss of lamin A/C results in growth retardation at 2 weeks post partum, with impaired postnatal hypertrophy of cardiac myocytes, skeletal muscle hypotrophy, decreased subcutaneous adipose tissue deposits, decreased adipogenic differentiation of mouse embryonic fibroblasts and metabolic derangements. Ultimately these tissue differentiation and maturation defects are lethal before mice are weaned. These results demonstrate that lamin $A / C$ is crucial in the early post-natal period and provides novel insights into the physiological function of A-type lamins in the context of the developing animal.

The significance of the results and their interrelationship are discussed in Chapter 7. The research in this thesis has started to unravel the role of lamin A and the effect of LMNA mutations in the context of disruption of proteinand chromatin interactions, chromatin organization and early postnatal tissue differentiation. These findings provide a solid foundation for ongoing research to determine disease mechanisms and ultimately potential cures for laminopathies. 


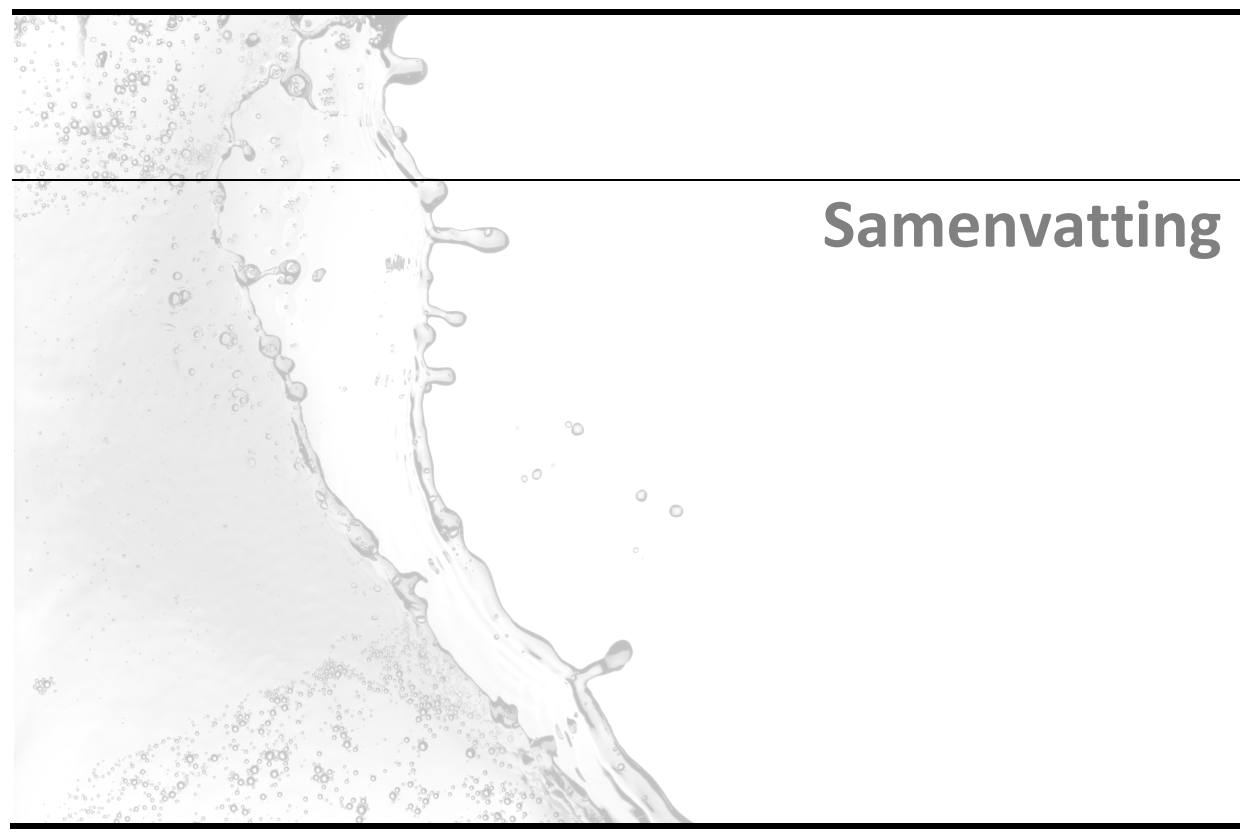


De nucleaire periferie wordt begrensd door de nucleaire envelop, die bestaat uit een buitenste en binnenste nucleair membraan, onderbroken door nucleaire porie complexen (NPC). De nucleaire lamina liggen aan de binnenkant van de nucleaire envelop en bestaan onder andere uit de intermediare filamenten A- (lamine A, C) en B-type lamines (lamine B1, B2), eiwitten van het binnenste nucleaire membraan die de lamina verankeren in de nucleaire envelop, en eiwitten die binden aan chromatine en de chromatine structuur modificeren. Mutaties in A-type lamines, geëncodeerd door het LMNA gen, veroorzaken verschillende humane ziektes, tezamen laminopathieën genoemd, waaronder enkele vormen van spierdystrofie, lipodystrofie, cardiomyopathie, neurologische afwijkingen en versnelde verouderings syndromen. Ten grondslag aan deze thesis ligt het centrale vraagstuk hoe mutaties in één gen zo'n grote phenotypische variëteit kunnen veroorzaken (Hoofdstuk 1). De werkhypothese van deze thesis is dat LMNA mutaties specifieke eiwit- en chromatine-interacties met de lamina beinvloeden, en daardoor laminopathie specifieke defecten worden veroorzaakt.

Hoofdstuk 2 beschrijft de meest voorkomende technieken die gebruikt worden om eiwit- en chromatine-interacties met de lamina te bestuderen, en bediscussiëerd de voor- en nadelen inherent aan elke methode. Aan het begin van het onderzoek uit deze thesis werden in vivo interacties van Atype lamines met chromatine gehypothetiseerd, maar moesten nog experimenteel bewezen worden. Methodologieën om eiwit interacties te identificeren waren sterk belemmerd door de zout- en detergentiaonoplosbare eigenschappen van de nucleaire lamina. Deze biochemische karakteristieken dwongen onderzoekers om een balans te vinden tussen de mate waarin eiwitten van de lamina in oplossing worden gebracht en de hoeveelheid interacties die mogelijk onderbroken worden door de gebruikte lysis condities. Alternatieve methodes om deze technische problemen te omzeilen hebben elk secifieke nadelen. Om de gebreken van bestaande methodes te boven te komen, hebben we een nieuwe techniek ontwikkeld, de OneSTrEP (OST) pull-down, om eiwit- en chromatine-interacties op een onbevooroordeelde manier proteoom en genoom-wijd te kunnen bestuderen. Deze techniek bestaat uit het expresseren van lamine A gelabeled met een op biotine lijkende OST epitoop, hoge affiniteits purificatie van volledig opgeloste maar intacte OST-lamine A complexen, en 
daarop volgende analyse van geïsoleerde complexen voor eiwitten met massa spectrometry en voor DNA met array technologie.

We hebben deze techniek gebruikt om eiwiten die binden aan lamine $A$ en progerine, een lamine A mutant die de versnelde verouderings ziekte Hutchinson-Gilford Progeria Syndroom (HGPS) veroorzaakt, te identificeren (Hoofdstuk 3). We detecteerden 150 lamine A en 170 progerine-bindende eiwitten, waaronder nieuw interactors van de nucleaire lamina. Lamine A interactors zijn bij voorkeur betrokken in structurele organisatie van de nucleaire envelop, chromatine assemblage en nucleair cytoplasmatisch transport via de NPC. Van de geïdentificeerde lamine A- en progerinebindende eiwitten, binden 35 bij voorkeur danwel lamine $A$ of progerine. Deze differentiëel bindende eiwitten bestaan o.a. uit transcriptie factoren, eiwitten met GTPase activiteit en eiwitten betrokken bij RNA metabolisme of nucleocytoplasmair transport. De identificatie van nieuwe lamine A- en progerine-interactors levert verschillende aanknopingspunten op voor studies naar specifieke interactie partners van wild-type en mutant lamine $A$, met als doel het ontrafelen van moleculaire mechanismes van weefselspecifieke laminopathieën.

Vervolgens hebben we genoom-wijd chromatine interacties van A-type lamines gemapt en identificeerden 595 lamine A- en 552 progerinegeassocieerde gen promoters (Hoofdstuk 4). We hebben vastgesteld dat lamine A bij voorkeur perifeer gelocaliseerde, inactieve genen bindt. Deze associatie faciliteert, maar is niet bepalend voor perifere localisatie en verlies van de binding is onvoldoende voor transcriptionele activatie van genen. Verdere analyse leidde tot een onderscheid in gemeenschappelijke en unieke lamine A- of progerine-bindende genen. Basale expressie van de unieke categorieën correleert met hun relatieve subnucleaire localisatie. Deze observaties demonstreren dat lamine $A$ en progerine op directe en verschillende wijze chromatine-lamina organisatie beïnvloeden en dat verlies van perifere localisatie en lamine-associatie van endogene gen loci onvoldoende is voor transcriptionele activatie.

Om de moleculaire basis voor premature verourderingsziekte karakteristieke defecten in chromatine structuur en accumulatie van persistente DNA schade te identificeren, hebben we een yeast two-hybrid 
screening uitgevoerd om differentiële lamine $A$ /progerine interactors te identificeren (Hoofdstuk 5). Rbbp4 en Rbbp7, onderdelen van het chromatine nucleosome remodelerings en deacetylase (NURD) complex, binden lamine $A$, maar niet progerine, en vertonen verlaagde eiwit niveaus tijdens normale en premature veroudering. Bovendien is de activiteit van histon deacetylase 1 (HDAC1), een andere component van het NURD complex, verminderd met veroudering. Inactivatie van verschillende NURD complex eiwitten recapituleerde verouderings geassocieerde chromatine defecten, die voorafgingen aan het ontstaan van persistente DNA schade. Deze bevindingen laten zien dat verlies van diverse NURD complex componenten plaatsvindt tijdens premature en normale veroudering en bijdraagt aan structurele chromatine defecten, uiteindelijk leidend tot de accumulatie van DNA schade.

Tot slot hebben we de biologische relevantie van A-type lamines in vroege postnatale weefsel differentiatie en maturatie onderzocht, relevant omdat LMNA expressie omgekeer evenredig is met de mate van differentiatie en verschillende $L M N A$ mutaties differentiatie defecten induceren. Met dat doel voor ogen hebben we een nieuw 'LMNA gene trap' (LMNA ${ }^{G T-/}$ ) muismodel gecreëerd, dat het het verlies van $L M N A$ functie combineert met een LMNA-gestuurde reporter gen expressie (Hoofdstuk 6). We tonen vroege activiteit van de $L M N A$ promoter tijdens het begin van embryonale differentiatie in hart, lever en somieten. Het verlies van lamine $A / C$ resuleert in groei retardatie 2 weken postnataal, met verzwakte postnatale hypertrophy van cardiale mycoyten, skeletspier hypotrofie, verminderde subcutane voorraden van adipoos weefsel, verminderde adipogene differentiatie van muis embryonale fibroblasten en metabole afwijkingen. Het eindresultaat van deze weefsel differentiatie en maturatie defecten is letaliteit, nog voordat muizen gespeend zijn. Deze resultaten bewijzen dat lamine $A / C$ cruciaal is in de vroege postnatale ontwikkeling en leveren nieuwe inzichten in de fysiologische functie van A-type lamines in de context van het ontwikkelende dier.

De significantie van de resultaten en hun onderlinge relatie worden bediscussiëerd in Hoofdstuk 7. Het onderzoek in deze thesis is begonnen met het ontrafelen van de rol van lamine $\mathrm{A}$ en de effecten van LMNA mutaties in de context van het verbreken van eiwit- en chromatine- 
interacties, chromatine organisatie en vroege postnatale weefsel differentiatie. Deze bevindingen leveren een solide fundament voor voortgaand onderzoek ter bepaling van ziekte mechanismes en, als ultiem doel, mogelijke genezing van laminopathieën. 
$204 \mid$ 


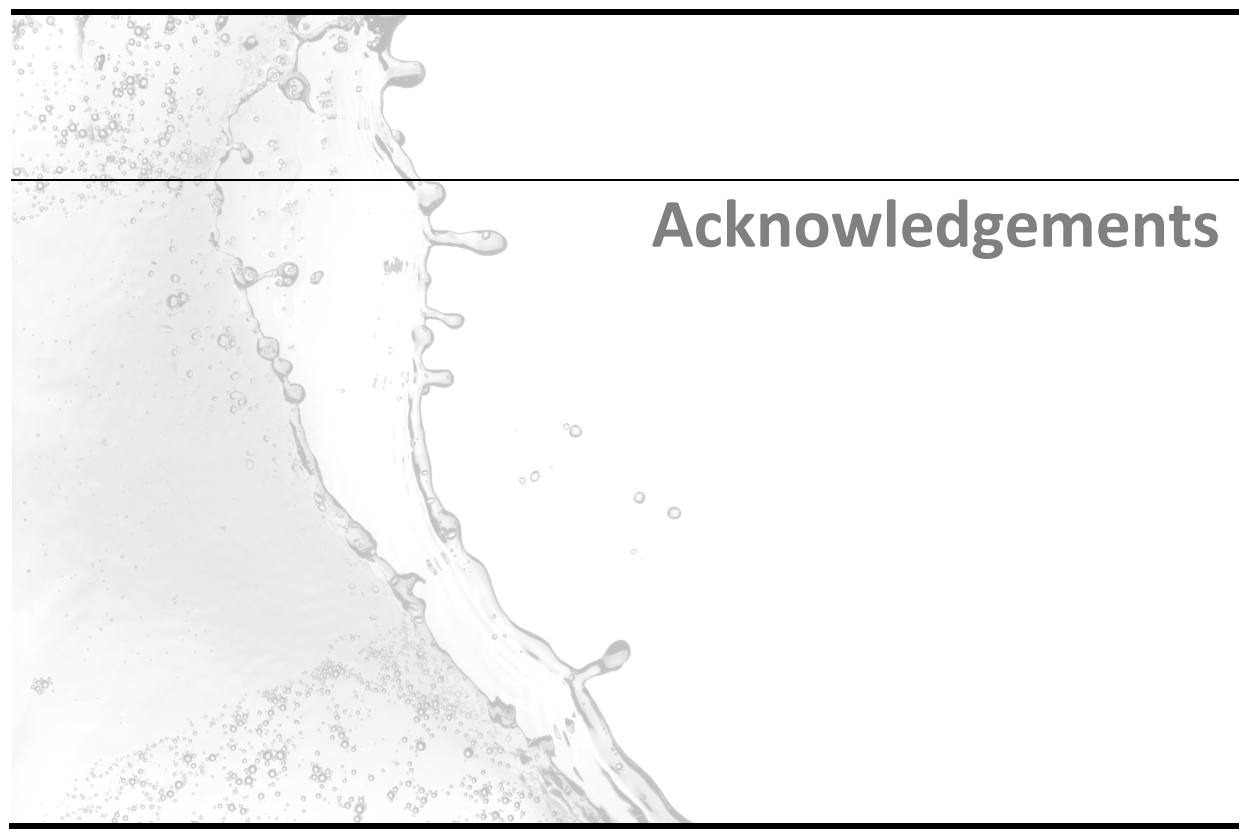


During the presentation of my university diploma my then supervisor and current co-promoter, Willem, speeched about how he had to discover for every new student which buttons to push in order to make them improve. He concluded that most times when he was about to push one of my buttons, I already had beaten him to it. Although one might conclude from this that I prefer to be in control of things, which I won't deny, the far more important interpretation, in my belief, is that I am fortunate to be surrounded by colleagues, friends and family who set many good examples for me to follow. It is my pleasure to put my appreciation for all of them here in words.

Dr. Voncken, Willem: You use your high analytical capacity and enthusiasm not only for scientific experiments but also to further motivate people around you. This demonstrates that you do not only have a passion for science but also for the people around you. You are a man of principles and live up to your promises, which was nicely demonstrated at my $\mathrm{PhD}$ job interview, where I answered the only two asked questions in the negative: "Do you live by yourself?"; "Would you like to spent a long time abroad to do research?". You promised Yigal to change my point of view on these matters and, somehow, did. Thank you for that!

Dr. Misteli, Tom: You set an example for me in the way you manage a laboratory and approach science. For both, with the greatest of ease and engagement, you have the ability to eliminate the unnecessary so that the necessary may speak. Even though I am only beginning to understand how to do that, I'm already enthusiastic about finding out much more about it, and am looking forward to working together the coming years. This will also provide me with the oportunity to convince you that age does not matter when attending a Tina Turner concert!

Prof. Dr. Pinto, Yigal: During our weekly meetings you complemented Willem's enthusiasm for basic science, with your emphasis on the clinical importance of research. Even though by times it was difficult for me to steer a middle course between the both of you, I've learned a lot from it. Becoming a self-dependent scientist most of all requires being able to make your own mistakes and learn from them. Your balance between taking action and 'laissez-faire' allowed me to do that. 
Prof. Dr. Crijns, Harry: Even though our encounters over the past years were brief, you always exuded genuine interest in what I was doing. I'm indebted to you for the financial support of my change-over to the $\mathrm{NIH}$. Furthermore I am grateful to you, Prof. Dr. Steijlen and Prof. Dr. Post for taking place in the thesis assessment committee.

My paranymphs. Geert, soon to become Dr. Tweezerman, side-by-side we infiltrated the Cardio lab with basic lamin research, and it is with great delight I look back at that period. Fortunately, you were not intimidated enough by my attempt to make you do a small dance on your university graduation, and soon after became a PhD-colleague and good friend. I admire your optimism and learned a lot from that positive perspective. Your Brabantian humor is widely appreciated and inimitable. I'll strive to introduce its concept within the US borders. A quick reminder to refresh your MMP-oriented brain: everything is about lamins (untill I start working on another topic)! The best of luck with your upcoming PhD defense. Melissa, we met during our study and after that always remained each others proximity. Not only did we learn from each other by sharing joys and sorrows, but also from our linguistic differences. It made me superior to Wikipedia in knowledge about the meaning of frou-frou! I am thankful for our friendship, your support as a paranymph during my thesis defense, and hope you'll find your niche in Milan and further career.

Fellow Cardio-ers: Gonda, you took on a lot of work for my PhD studies, especially regarding the LMNA genetrap mouse and the ever memorable million dollar mouse. Thereby your contribution was crucial for the realization of this thesis, and I am very thankful to you for that and all the nice moments we had while working on these projects. Chapter 6 is yours! Joost, your social attitude was crucial for the Cardio atmosphere. Blanche's and Mark's PhD movies capture that spirit brilliantly. Going together to the Dutch-German Joint Cardiology meetings, the Keystone Symposium with a detour through Denver would not have been as much fun without you. Good luck with your thesis defense! Rudy, Mr. Duitsers, your attempts to put my world upside-down, figuratively sometimes literally, were effective and are greatly appreciated. One question remains open: Who finished first in Nice? Oh well ...we both have our answer to that question. The best of 
luck with your new job. Stephane, even though the focus of my projects was on the cell nucleus, you broadened that narrow perspective with enthusiasm by getting me acquainted with the world of matricellular proteins. Thank you for that! Rick, your timing of saying "Out of sight, out of mind" is unrivaled. With that witticism you make colleagues quickly feel comfortable at the lab and open up socially. I am thankful for that and your support in giving guidance to students. Wouter, your experience as a technician is rapidly growing, and makes you an important factor within the lab. Your humor can be as dry as Geert's, and personally I still can't stop smiling when thinking back at your 'Darth Vader' joke. Blanche, your talent especially shows in the critical questions you ask during meetings, your ability to go around with many different personalities, networking qualities, and the skill to carefully build up a scientific career next to a family. You thereby set an example for me. M.A.R.K., no one can carry out Brabantian pride and joviality as much as you. You earned me my nickname, introduced the concept of intense clown, and took the preliminary work on you for introducing a new hazard label on fixative bottles "Dangerous in combination with extreme Italian shoes". It is now waiting for a safety sign to be put on you: "Contagious humor". Thank you for your help with the animal work and good luck with your new job! Arie, you introduced me and many colleagues into the world of micro-arrays, which soon gave you the honorable nickname of "micro-Arie". Your knowledge was vital for this thesis, especially Chapter 6. Esther, you have vision as you realized well before I did, that I should go abroad to do science. It is re-assuring having seen you walk the same path and the other way around as well successfully. My door is open for more predictions! Viola, you recently changed your work- and life-style completely by making a change-over to South America. I admire the noble intentions behind that choice. Jop, my predecessor in the lamin research. Is performing large-scale experiments a necessity in lamin research or is it simply an ingrained habit of the both of us? I wish you all the best in your further career. Anke, Inge and Wino: It is good to see/hear how you all are in your element at the AMC. I wish you all the best. Suzanne, Veronica, Mirjam and Kim: Thanks for all your support! The department of Cardiology's secretaries: Miriam, Bianca, and especially Barbara, thank you very much for making everything run smoothly from an organizational point of view. Barbara, I realize that lately I've come short on delivering you new stories to read in the train, hopefully I made it up to you with this thesis! 
Not to be overlooked are the many Cardio-interns who each have contributed their fair share to this thesis over the past years. Lieke, Thijs, Rayna and Cynthia: With the best of intentions I've asked a lot of dedication from you, and I am very thankful to have had the opportunity to learn from all of you about the ins and outs of guiding students. Frank, you are spreading out the Cardio spirit across university. Good luck with that! Kevin, the English dictionary does not contain the word 'Sjeng'. They should introduce it with a picture of you next to it! A special word of thanks goes to Chantal. Your fanaticism in learning, discovering and working amazed me. With that attitude I'm positive that you will not only do great in science, but also in many other things you wish to accomplish in life. It is my absolute pleasure to reward your hard work with two co-authorships.

Just as there is no true South Limburg without the idyllic Munstergeleen there is no heart-related research without Molecular Genetics. When I started working at the Cardiology department, I often found myself walking up the stairs to the fifth floor subconsciously. Is that epigenetic imprinting? It is with much pleasure I look back at my internship at Molgen and the close association afterwards. Hanneke, you thought me the basics of the molecular biology; not only techniques, but also your mantra "All shall be well", which is a helpful motivation when experiments fail. I'm looking forward to giving you a tour through Washington, and you expanding my extraordinary limited knowledge about Baltimore! Frank, your optimism only has one setting, maximal. Even when having to withstand all my whistling you had a smile on your face; impressive! Peggy, you have extended the proportions of your experiments beyond science, considering your latest project: The Prickaerts Palace. Even though we have never directly worked in the same lab, it instantly felt like we did when we met, thanks to the pleasant chats. I enjoy the get-togethers with Hanneke, Frank and you, and hope they will continue! Vivian, a model of diligence. Not often do I see someone who works as accurate and organized as you do. I've learned a lot from your example and am thankful for your help on the animal work. Regarding the LMNA genetrap mice I also want to thank Marjon, who contributed her expertise to help solving unanswered questions. Iris and Céline, thank you for your contribution to the nice atmosphere durning my Molgen internship. Claudia, we got thrown in at the 
deep end together. You are always friendly and willing to help out, thank you for that.

The warm welcome I received when coming to the NIH in Bethesda, was not only due to the subtropical climate, but especially due to the many great colleagues and friends. The pleasant atmosphere you all helped creating was crucial in making my experience abroad to a success. Not only did it make me learn a lot inside the lab, it also created many valuable experiences outside of the lab, allowing me to grow as a person. I am looking forward seeing you all again and continue working with you for the next years! In random order: Karen, you are a caring, ever-social and thereby central factor within the lab. Furthermore a guard (or is it occupier?) of my lab bench and worthy sparring partner in sarcasm. The latter I only show when feeling really comfortable around someone, so consider the blank pages within this thesis and other sarcasm as a great compliment for you in disguise! Christine, that same disguise applies on the many German-related jokes which are very hard for me to hold in around you. You are a hard-working, bright and cheerful bench mate, who furthermore has the virtue of not gossiping but always pointing out the positive aspects of people around you. I admire that. Nico, even though our characters are fairly opposite, we not only respect those differences, it even served as a breeding ground for many joyful adventures experienced together. How can I forget the evening we cooked risotto with almost forgetting the rice, the after midnight goulash, the visit to Baltimore, baseball lessons with a broomstick, pursuing a police/robber-chase in NYC, your well meant step-by-step guidance later that night at the crashed birthday party and our endless discussions on where to cross the road and how to walk back to Melvern Drive. You always took me in tow on your pursuit of liveliness throughout D.C. and I am very thankful to you for making me grow as a person by that. You only created one fear response in me, which becomes evident when I hear this sentence: "Don't worry!". We should work on that when I visit you in Buenos Aires. Alex, you have neverending optimism. As only to be expected from a true Frenchman you came up with a very original way to transport wine glasses. I'll never forget your farewell drinks at the Harp \& Fiddle. Thank you for getting me acquainted with animal work at the $\mathrm{NIH}$, and I wish you the best of luck with further setting up your own lab in Lyon. Gianluca, an Italian gentleman of wide 
reading and in addition a modest person. Those are great qualities! You provided me with the opportunity to work together on a revision experiment of your research project, by far the most appealing chapter of this thesis. Thank you for that! Nisha, always smiling and a very social person. You once told me I was absorbing the cooking-skills going on in your kitchen as a sponge. I hope to be able to do that sometime again in the near future, since the Indian cuisine has so many mysteries to me. Vassilis, your semi-grumpy remarks are funny, Malaka! You have the true Greek spirit of knowing what great dishes Hellas harbors, and together we also found out how to prepare some of them, with use of every single piece of kitchen equipment and some furniture (I remember eggplant being put in vases). Your big-heartedness nicely showed when one of those evenings you looked after me by telling me to leave the cooking to you and go talk to Uli, also seeing to it that I did. I look back at those 'Greek' evenings with much joy and hope we can experience some more. Reini, the artistic free-spirited Spanish influence of the lab. Whenever I think I have been to most of the interesting places in Bethesda and D.C., it is only that I have to hear you talking about the city and realize there are so many more interesting places I still have to go discover within the city. You published an excellent scientific paper recently, and I am sure there are more to follow! Beck, you always seem to introduce me with concepts I did not know before; a random selection: block parties, your own-mixed herbal drink, Tom Yum soup, using rosewater in cakes, a werewolves card-game, old P.T.T. jackets in the U.S., 4th of July celebration with fireworks. You do that in an open/hospitable way, which I like! Steve, you are not only making things run smoothly at the lab, but also update me during tissue culture on American musical history, which I still have to learn a lot about. I like the twinkle in your eyes, when you start talking about $\mathrm{BBQ}^{\prime}$ ing with a spirit that almost makes it feel like a form of art! That is a true Chef's way of talking about cooking, and it makes me enthusiastic (and hungry) every time. Travis, you interpret 'translational science' in your own way, by experimenting with yeast to bake bread. Despite your hard work you remain mellow, which makes you a very approachable person. I hope all the yeast-work will result in nice data for you! Sarah, always polite; the continuous force on the 'progeria drug targets screening'-project. It is nice to have you as a colleague and I'm keeping my fingers crossed for an effective compound to roll out of the screening. Ty, can do the magic tricks with computers, to make happen what others 
suspect is possible, but have no idea about how to effectuate it. Such knowledge is not only impressive, it is vital in making large scientific adventures to a success. Saying Paola, immediately makes the word talent come to my mind. Your fanaticism in discovering, connecting untied ends and doing experiments sets a standard for the lab to strive for. I admire that greatly, and am looking forward to working with your support on the RNAi screening project. Patricia, our paths only crossed shortly at the end of my stay, I wish you all the best. Simon, an LRBGE/CBGE hybrid, superior to me in organization drive. Although I know you don't agree with me on the latter, I'll stick to the argument that sorting pens by color, thickness of the tip and many other unknown criteria, beats sorting bottles with chemical solutions into a few labeled categories. You are always up for a good exchange of sarcastic remarks, and unique were the men's nights, formerly known as date-night's. I wanted to say unforgettable but changed that to unique, because there seem to be some gaps or fuzzy moments in my memory. What I do remember are the new-and-improved flambéed chicken oven-dish, the watermelon to go with that, jello dessert, special ice-tea and an exhaustive lord-of-the-rings marathon. We had quite a good bad influence on each other, thank you for that! Good luck with your (carefully planned) career! And then one men's night there was not only Rambo but also lady Gaga. Pilar, you are always up for liveliness accompanied by a catchy laugh. A mentor in American slang, guide from New Jersey's White Castle, Applebee's to excellent Filipino food, and colleague who has exchanged the U.S. for Europe. I wish you all the best in your PhD! Keren, always in a good mood, very friendly and with an open personality. You generated strange habits in me, not only was I intrigued by how your lunch looked like, since you always took the effort to cook your own food and frequently let me try a bit; I also found myself automatically checking whether you were in the Opera room when passing by, because I liked stopping by for a chitchat. I'll miss that. I wish you all the best!

Next, there are many people outside of the departments mentioned above, who I would like to acknowledge. To start with the Toxicology department, the place where I got acquainted with scientific work during my studies. Agnes, I like to thank you for your moral support throughout the years, which you always give with trust in me and without judging me. I enjoy how all those years after my internship we still remain in good contact and 
witness important steps in each other's life's. I'll do my best to keep feeding your longing for the U.S. with new stories! I wish you and Tim a happy, harmonious and joyful togetherness for life. Guido, you once said that selfcritique is the most difficult thing to give. Although it didn't make it to my final eleven propositions, that statement always appealed very much to me. Thank you for your guidance during my period at Toxicology. From the department of BigCat: Michiel, you have performed a great deal of work on the ChIP project, and always proved a very helpful, enthusiastic and a reliable colleague in analyzing the data that were generated. It is a pleasure to reward that work in a co-authorship, and I hope we can further collaborate on future projects! Next, continuing to Population Genetics, I would like to thank An and Bianca, as well as Kees from the Erasmus Medical Center, for their help on the mitochondrial characterization of the LMNA genetrap mouse. Since the phenotype of that mice is mysterious the project soon became an inter-departmental one, and I would also like to take this opportunity to thank Hans for the electron microscopy analysis, and Ben, Jacques, Peter, Agnieszka, Helma, Nicole for their help and guidance in the animal work. A special word of thanks also for Paulien, Richard and Hub, from the central animal facility, who always where willing and helpful to create good logistics for these experiments. From the AMC, Ronald and Sander: you received me in an open and spontaneous way when I asked to collaborate on the LMNA genetrap mouse. Metabolomics is a fully unknown territory for me and your help in analyzing the metabolic phenotype was crucial for Chapter 6. Jeroen, our collaboration on the mass spectrometry analysis of lamin protein-interactions is the foundation of Chapter 3. I appreciate your help on this, and hope to extend together on this project in the future. Lastly, Andrea, I appreciate how we always could exchange our thoughts, frustrations and hopes on the ChIP experiments. Even though the places where we got to meet each other are a bit out of the ordinary (the MolGen corridor, a pub in Washington, a wedding, a housewarming party), I always enjoy whenever we bumped into each other and $I$ experience your and Sofia's warm- and open-mindedness.

Mijn laatste stelling geeft aan dat het belangrijk is om afstand te kunnen nemen van de wetenschap, en de zwemclub staat daarbij al jarenlang voorop. Waar in de wetenschap geleerden gedreven leren, is er de wetenschap dat Watervrienden-gelederen leren drijven. Dat brengt altijd 
heel veel plezier met zich mee, zeker als dat drijven wordt uitgebreid tot zwemmen, even bijkomen van de geleverde inspanning, kletsen aan het einde van de baan, aan de kant, de douches, het kleedlokaal, het sporterscafé en op BBQ's. Graag wil ik dan ook Roel, Theo, Michel, Dion, Ansel, John, Kim, Carla, Claudy, Bianca, Linda, Emma en Francoise van Baan $4 / 5$ en andere Watervrienden bedanken voor deze gezelligheid. Dat er nog veel mooie 'strijd' om, en vooral uitreikingen van (troost)prijzen mogen volgen! Roel, je hebt altijd het hart op de tong, hetgeen ik waardeer, net als de avonden waarop wij over het bovenstaande eindeloos na kunnen kletsen. Veel geluk voor jou en Samira!

Dennis, stelling tien heeft alles te maken met zelfreflectie en dit durven te delen met anderen. Jij kunt dat, hetgeen onder andere blijkt uit het dankwoord van je scriptie. Dat brengt me tot stelling negen: je pretendeert niet, maar bent iemand: een goed luisteraar, bij tijd en wijle filosoof, en bovenal een zeer goede vriend! Het blijft alleen eeuwig zonde dat je nu juist uit Stein komt. Roy, Jaap, Jan, Robbert en Laurens, ondanks dat we allemaal druk bezig zijn met onze levens op te bouwen door het hele (buiten)land, is er toch ook altijd tijd om weer terug bij elkaar te komen, en al onze belevenissen te delen met elkaar. Dat voelt vertrouwd en doet ook veel deugd. Dank jullie voor deze vriendschap!

Ulrike, Miss Punt, enjoying the simple things simply together is what I cherish most. I am greatly indebted to you for making me understand that sometimes not trying to understand, but to feel, feels ... beautifUli. Remember this one-liner: in whatever two places we will be, we will come into line.

Pieter, dat het mogelijk is moleculair biomedisch onderzoek te doen zonder p53 direct erbij te betrekken blijkt uit dit proefschrift. Biomedisch onderzoek doen zonder jouw betrokkenheid is echter lastiger. Al van jongs af aan hebben we een goede band en sta je altijd klaar om bij te springen daar waar hulp nodig is (P.S.: in het cursief de Engelstalige essentie van je huidig beroep ;) ). Dit boekje is daarom ook een beetje van jou! Pap en mam, hoe ouder ik word hoe meer ik begin te begrijpen dat jong geleerd, oud gedaan is. Als ik probeer de essentie van een goede opvoeding te verwoorden, dan is dat het aanleren van een zelf-lerend vermogen. Dat 
vermogen maakt dat je niet hoeft te lijken, maar bepaalt wie je bent en daar zelfverzekerd in bent (stelling 9 en 10). Zelf-lerend vermogen is niets anders dan het voor jezelf kunnen indrukken van de juiste knoppen, en daarmee staat het grootste compliment voor de opvoeding die jullie mij hebben gegeven reeds vermeld in de eerste alinea van dit dankwoord. Mijn dank is groot voor jullie niet aflatende steun! Naast dat jong geleerd, oud gedaan is, geldt voor mijn opvoeding ook dat jong gedaan, oud geleerd is: Jullie hebben mij ooit als kind wijsgemaakt dat bij drie littekens op mijn hoofd ik Professor zou worden. Hierbij in ieder geval een stapje in die richting! 
$216 \mid$ 


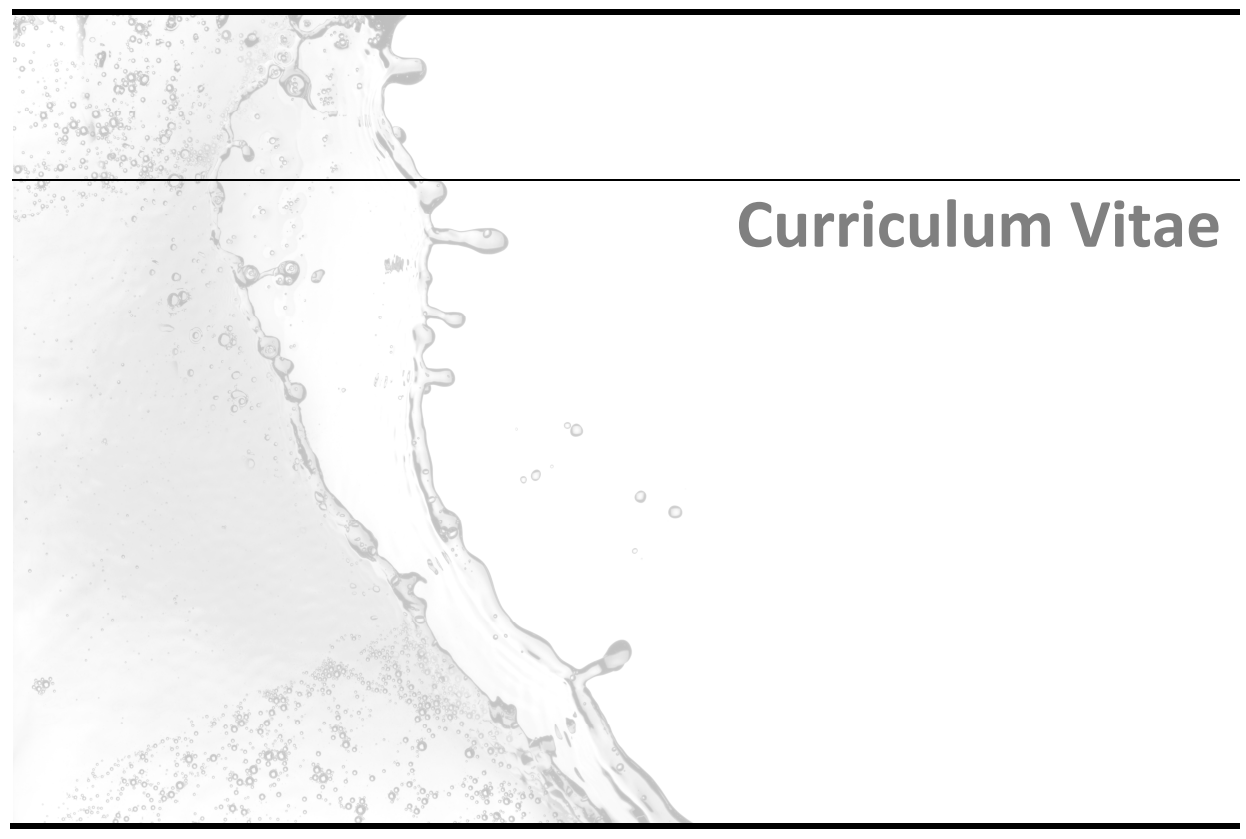


Nard Kubben was born December $18^{\text {th }}, 1981$ in Geleen. He attended the Graaf Huyn College in Geleen, where he graduated in 2000. The same year, he started to study Health Sciences at Maastricht University. In his fourth year, he performed an internship at the department of Molecular Genetics on the effects of 'Mitogen-activated protein kinase'-induced phosphorylation on Polycomb Group localization, which he was nationally awarded for with the Unilever Research Price in 2005. After graduation in Biological Health Sciences in 2004, he started his PhD at the department of Cardiology at Maastricht University with a focus on the identification of nuclear lamina protein- and chromatin-interactions and their respective roles in laminopathies. In 2007 he won the poster prize at the $5^{\text {th }}$ Dutch German joint meeting of molecular cardiology groups. In the last year of his $\mathrm{PhD}$ he continued his work at the National Institutes of Health (NIH) in Bethesda (USA) and was rewarded with a fellows award for research excellence in 2009. He will continue his work on laminopathy disease mechanisms by starting a post-doctoral training at the $\mathrm{NIH}$. 


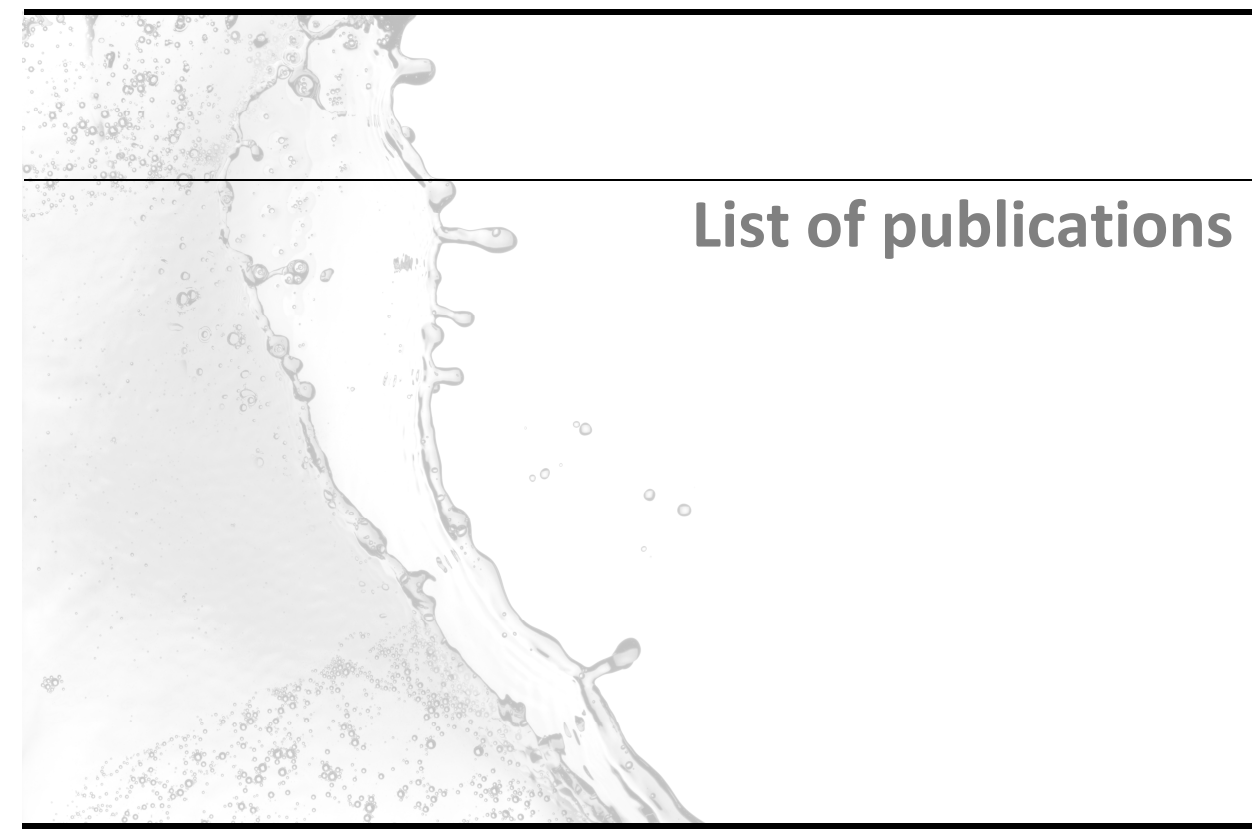


1. Kubben N, Adriaens M, Voncken J, Pinto Y, Misteli T. Identification of lamin Aand progerin-interacting genome regions. 2010. In preparation

2. Kubben $\mathbf{N}^{*}$, Voncken $J^{*}$, Konings $G$, van Weeghel $M$, Gijbels $M$, van Erk $A$, Schoonderwoerd K, van den Bosch B, Dahlmans V, Calis C, Houten S, Misteli T, Pinto Y. Post-natal myogenic and adipogenic development defects and metabolic impairment upon loss of A-type lamins. 2010. In preparation

3. Kubben N, Voncken J, Demmers J, Calis C, van Almen G, Misteli T, van Almen $G$, Pinto $Y$, Misteli T. Identification of differential protein interactors of lamin A and progerin. Nucleus. 2010 Dec. Accepted

4. Kubben N, Voncken J, Misteli T. Mapping of protein- and chromatininteractions at the nuclear lamina. Nucleus. 2010 Dec. Accepted

5. Schellings $\mathbf{M}$, Vanhoutte D, van Almen G, Swinnen $\mathbf{M}$, Leenders J, Kubben $\mathbf{N}$, et al. Syndecan-1 amplifies angiotensin II-induced cardiac fibrosis. Hypertension. 2010 Feb;55(2):249-56.

6. Pegoraro G, Kubben N, Wickert U, Gohler H, Hoffmann K, Misteli T. Ageingrelated chromatin defects through loss of the NURD complex. Nat Cell Biol. 2009 Oct;11(10):1261-7.

7. Schroen $B$, Leenders J, van Erk A, Bertrand A, van Loon $M$, van Leeuwen $R$, Kubben N, Duisters R, Schellings $M$, Janssen B, Debets J, Schwake M, Hoydal $M$, Heymans $S$, Saftig $P$, Pinto $Y$. Lysosomal integral membrane protein 2 is a novel component of the cardiac intercalated disc and vital for load-induced cardiac myocyte hypertrophy. J Exp Med. 2007 May 14;204(5):1227-35.

8. Van Berlo J*, Voncken J*, Kubben N, Broers J, Duisters R, van Leeuwen R, Crijns $\mathrm{H}$, Ramaekers $\mathrm{F}$, Hutchison $\mathrm{C}$, Pinto $\mathrm{Y}$. A-type lamins are essential for TGF-beta1 induced PP2A to dephosphorylate transcription factors. Hum Mol Genet. 2005 Oct 1;14(19):2839-49.

9. Voncken $\mathrm{J}^{*}$, Niessen $\mathrm{H}^{*}$, Neufeld $\mathrm{B}^{*}$, Rennefahrt $\mathrm{U}$, Dahlmans $\mathrm{V}$, Kubben $\mathbf{N}$, Holzer B, Ludwig S, Rapp U. MAPKAP kinase 3pK phosphorylates and regulates chromatin association of the polycomb group protein Bmi1. J Biol Chem. 2005 Feb 18;280(7):5178-87.

10. Boots A, Kubben N, Haenen G, Bast A. Oxidized quercetin reacts with thiols rather than with ascorbate: implication for quercetin supplementation. Biochem Biophys Res Commun. 2003 Aug 29;308(3):560-5.

(*equal contribution) 


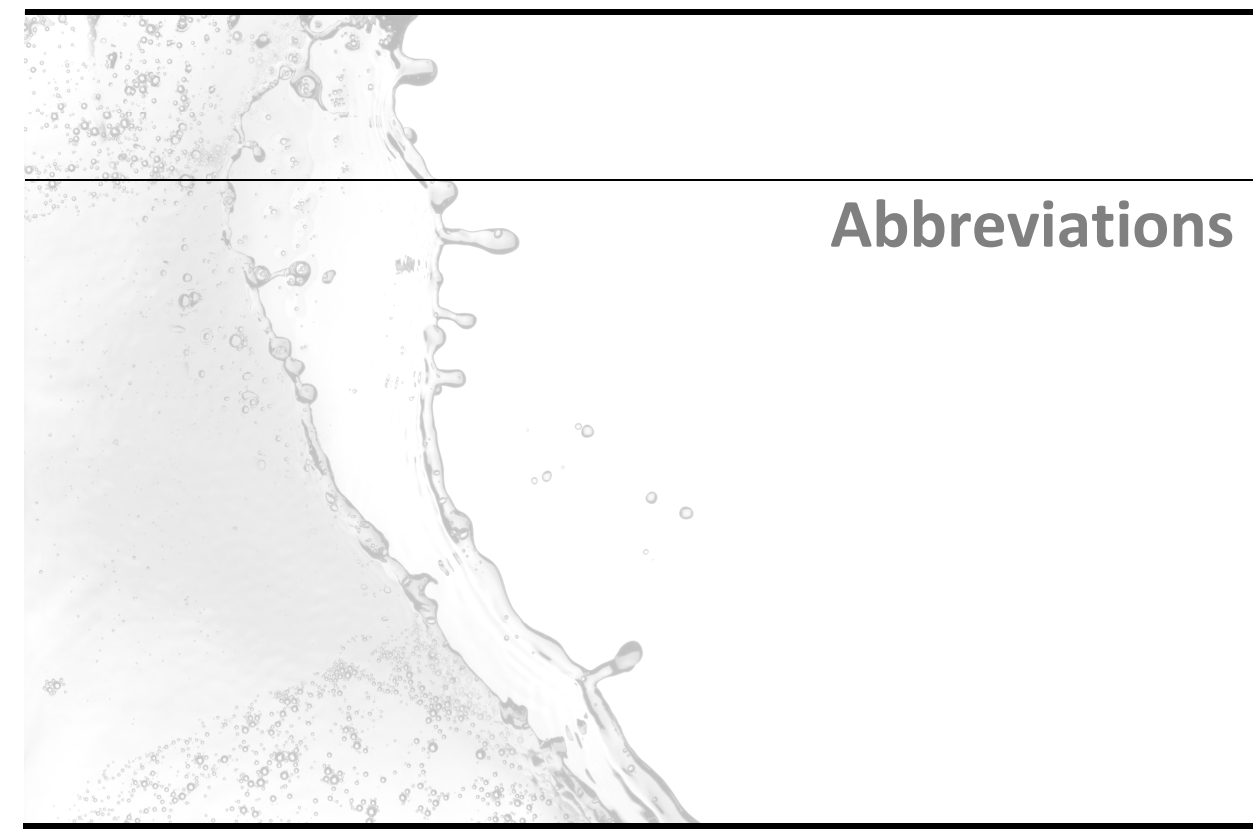




\begin{tabular}{ll} 
AHCTF1 & AT hook containing transcription factor 1 \\
ANF & Atrial natriuretic factor \\
BAC & Bacterial artificial chromosome \\
BNP & Brain natriuretic peptide \\
BPTF & Bromodomain and plant homeo domain transcription Factor \\
BRN5 & Brain-5 \\
BW & Body weight \\
CBB & Coomassie brilliant blue \\
CEAS & Cis-regulatory element annotation system \\
ChEC & Chromatin endogenous cleavage \\
ChIC & Chromatin immunocleavage \\
ChIP & Chromatin immunoprecipitation \\
CMD1A & Dilated cardiomyopathy 1A \\
CS & Citrate synthase \\
DAVID & Database for annotation visualization and integrated discovery \\
DCM & Dilated cardiomyopathy \\
DCr & Decorin \\
DN & Dominant negative \\
ECG & Electrocardiogram \\
ECM & Extracellular matrix \\
EDMD & Emery-Dreifuss muscular dystrophy \\
ER & Endoplasmic reticulum \\
FC & Fold change \\
FFA & Free fatty acids \\
FPLD & Dunnigan familial partial lipodystrophy \\
FS & Fractional shortening \\
GAPDH & Glyceraldehyde 3-phosphate dehydrogenase \\
GO & Gene ontology \\
GPC3 & Glypican \\
GT & Gene trap \\
H\&E & Hematoxylin and eosin \\
HDAC1 & Histone deacetylase 1 \\
HE & Heterozygous \\
HGPS & Hutchinson-Gilford Progeria syndrome \\
HPRT & Hypoxanthine-guanine phosphoribosyl transferase \\
IBMX & 3-isobutyl-I-methylxantine \\
\hline &
\end{tabular}




\begin{tabular}{|c|c|}
\hline IFNp & Interferon gamma \\
\hline $\mathrm{IHC}$ & Immunohistochemistry \\
\hline INM & Inner nuclear membrane \\
\hline IP & Immunoprecipitation \\
\hline IVSd & Intraventricular septal wall thickness in diastole \\
\hline IVSs & Intraventricular septal wall thickness in systole \\
\hline $\mathrm{KI}$ & Knock-in \\
\hline KO & Knock-out \\
\hline LAD & Lamin-associated domain \\
\hline LMNA $^{-/-}$ & Homozygous LMNA knock-out \\
\hline LMNA $^{+/+}$ & LMNA wild-type \\
\hline LMNA $^{\mathrm{GT}-/-}$ & Homozygous LMNA genetrap \\
\hline $\operatorname{LMNA}^{\mathrm{GT+}+-}$ & Heterozygous LMNA genetrap \\
\hline LMNA $^{\mathrm{KO}-/-}$ & Homozygous LMNA knock-out \\
\hline LSB & Laemmli sample buffer \\
\hline LV & Left ventricle \\
\hline LVIDd & Left ventricular internal dimension in diastole \\
\hline LVIDs & Left ventricular Internal dimension in systole \\
\hline LVPWd & Left ventricle posterior wall thickness in diastole \\
\hline LVPWs & Left ventricle posterior wall thickness in systole \\
\hline LVW & Left ventricle weight \\
\hline MAD & Mandibuloacral dysplasia \\
\hline MEF & Mouse embryonic fibroblast \\
\hline MS & Mass spectrometry \\
\hline NE & Nuclear envelope \\
\hline NPC & Nuclear pore complex \\
\hline NURD & Nucleosome remodeling and deacetylase \\
\hline NUSAP1 & Nucleolar and spindle associated protein 1 \\
\hline OCT-1 & Octamer transcription factor 1 \\
\hline OLFR & Olfactory receptor \\
\hline ONM & Outer nuclear membrane \\
\hline OST & OneSTrEP \\
\hline OST-A & OneSTrEP tagged lamin A \\
\hline OST-C & OneSTrEP tagged lamin C \\
\hline OST-P & OneSTrEP tagged progerin \\
\hline PAS & Periodic acid shiff \\
\hline
\end{tabular}




$\begin{array}{ll}\text { Pcna } & \text { Proliferating cellular nuclear antigen } \\ \text { PCP } & \text { Protein correlation profiling } \\ \text { PHD } & \text { Plant homeo domain } \\ \text { PP } & \text { Post partum } \\ \text { Prx1 } & \text { Paired related (mesoderm) homeobox } 1 \\ \text { RP58 } & \text { Repressor protein with a predicted molecular mass of } 58 \mathrm{kDa} \\ \text { SDS } & \text { Sodium dodecyl sulfate } \\ \text { SR } & \text { Picro Sirius Red } \\ \text { Srebp1 } & \text { Adipocyte differentiation factor sterol element binding protein 1 } \\ \text { SRF } & \text { Serum responsive factor } \\ \text { TALE } & \text { Three-amino acid-loop extension } \\ \text { TEM } & \text { Transmission electron microscopy } \\ \text { TF } & \text { Transcription factor } \\ \text { TFM } & \text { Transcription factor binding motif } \\ \text { Tpr } & \text { Translocated promoter region } \\ \text { VMNR } & \text { Vomeronasal receptor } \\ \text { VSMC } & \text { Vascular smooth muscle cell } \\ \text { WT } & \text { Wild-type } \\ \text { Y2H } & \text { Yeast two-hybrid }\end{array}$


Colour figures | 225

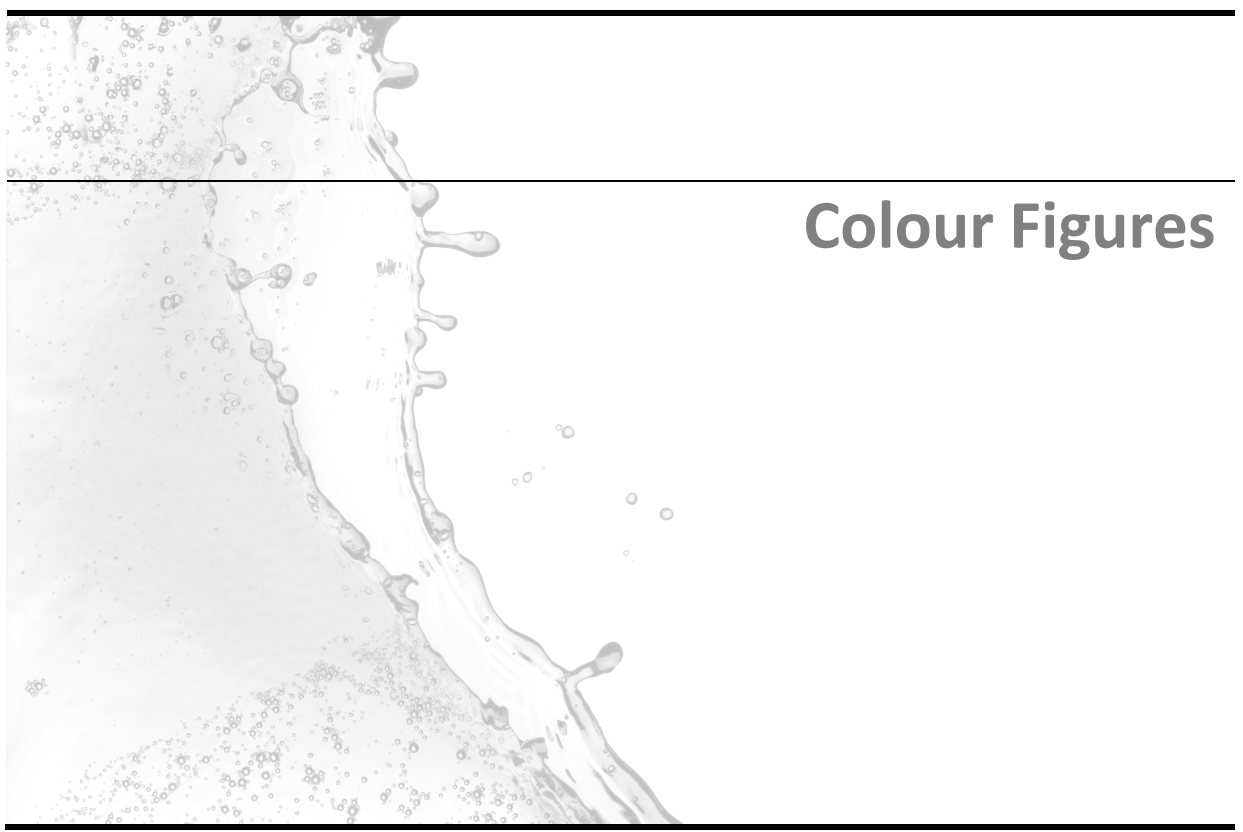


A

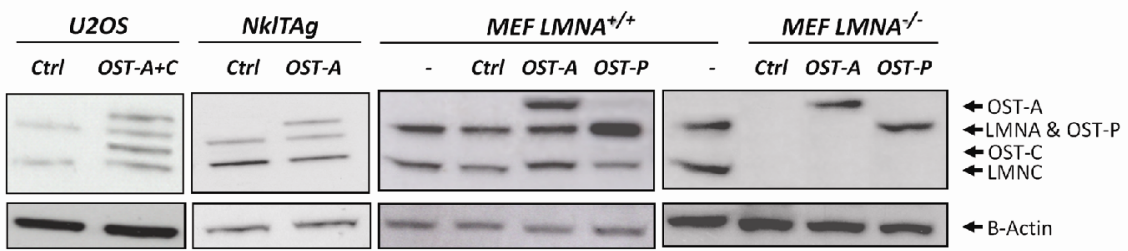

B

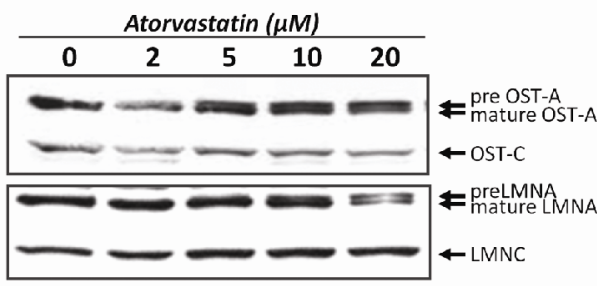

C

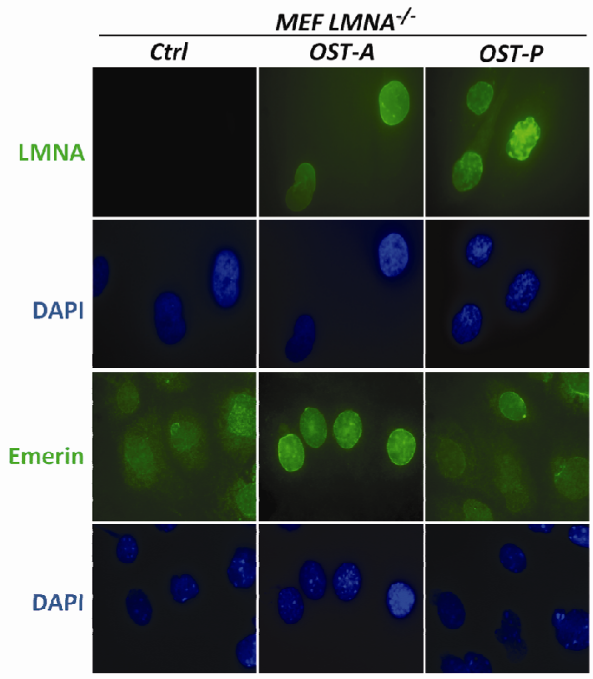

D

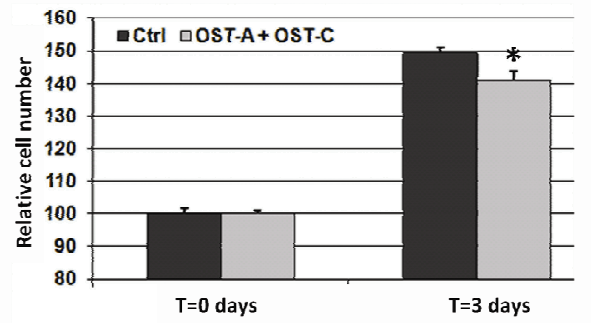

Chapter 3; Figure 1

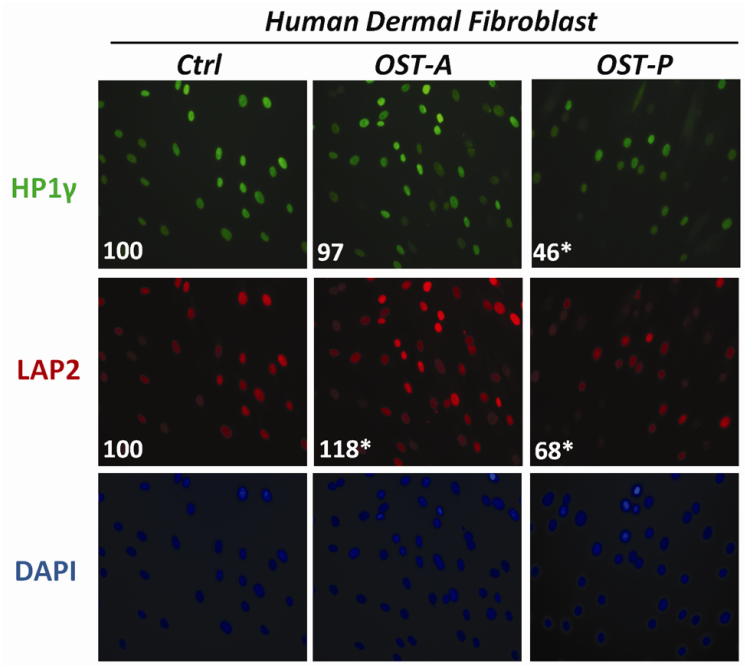

Chapter 3; Figure S2 

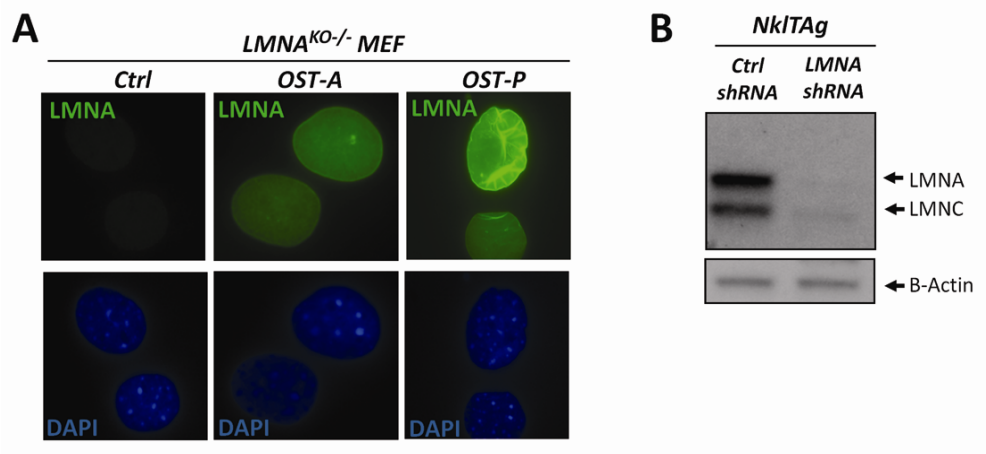

\section{Chapter 4; Figure S1}
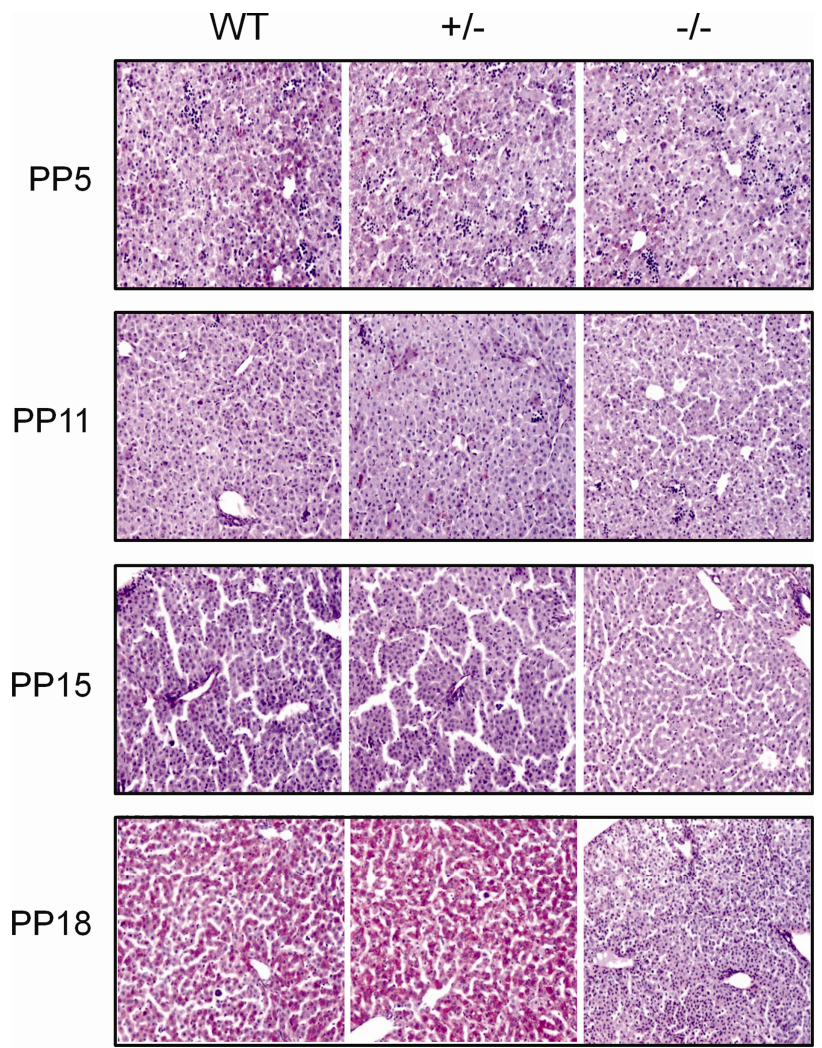

Chapter 6; Figure S2 

Let us try to teach generosity and altruism, because we are born selfish.

- Richard Dawkins - 


